\title{
A TOY MODEL FOR THE DRINFELD-LAFFORGUE SHTUKA CONSTRUCTION
}

\author{
D. GAITSGORY, D. KAZHDAN, N. ROZENBLYUM AND Y. VARSHAVSKY
}

\begin{abstract}
The goal of this paper is to provide a categorical framework that leads to the definition of shtukas à la Drinfeld and of excursion operators à la V. Lafforgue. We take as the point of

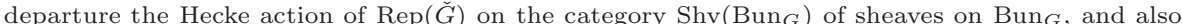
the endofunctor of the latter category, given by the action of the geometric Frobenius. The shtuka construction will be obtained by applying (various versions of) categorical trace.
\end{abstract}

\section{CONTEnts}

Introduction

0.1. Hecke action

0.2. What is done in this paper?

0.3. Organization of the paper

0.4. Notation and conventions

0.5. Acknowledgements

2

1. Symmetric monoidal categories integrated over a space

1.1. The integral

1.2. The tensor product $\mathcal{A}^{\otimes Y}$ as a colimit

1.3. Proof of Theorem 1.2.4

1.4. The category of local systems

1.5. Digression: the stack of G-local systems

1.6. Proof of Theorem 1.5.8

1.7. Functors out of $\mathcal{A}^{\otimes Y}$ as diagrams parameterized by finite sets

1.8. Objects in and functors out of $\mathcal{A}^{\otimes Y}$

2. Excursions

2.1. Description of $\mathcal{A}^{\otimes Y}$ via the fundamental group

2.2. Proof of Proposition 2.1.5

2.3. Digression: affine functors

2.4. Endomorphisms of the unit object in $\mathcal{A}^{\otimes Y}$ as a colimit

2.5. Proof of Proposition 2.4.6

2.6. Applying the paradigm 34

2.7. Endomorphisms of the unit, term-wise $\quad 35$

2.8. Action on a module via excursions $\quad 38$

2.9. Proof of Theorem 2.8.7

3. Taking the trace $\quad 42$

3.1. The usual trace $\quad 42$

3.2. Trace in a 2-category $\quad 43$

3.3. Properties of the 2-categorical trace $\quad 46$

3.4. Trace on DG categories $\quad 47$

3.5. Examples $\quad 49$

3.6. Trace on DG 2-categories $\quad 53$

3.7. The 2-categorical trace and (categorical) Hochschild chains $\quad 56$

3.8. The 2-categorical class map $\quad 57$

Date: February 8, 2022. 
3.9. A framework for the proof of Theorem 3.8.5

3.10. Proof of Theorem 3.8.5 isomorphism of the underlying objects of Vect 16

3.11. Proof of Theorem 3.8 .5 algebra and module structure $\quad 63$

3.12. A more elementary proof of Theorem 3.8 .5

4. A few mind-twisters $\quad 68$

4.1. The class of a class $\quad 68$

4.2. Proof of Proposition 4.1.6 $\quad 69$

4.3. The "trivial" case and excursions $\quad 72$

\begin{tabular}{lll} 
4.4. & Introducing observables & 74 \\
\hline
\end{tabular}

4.5. Cyclicity and observables $\quad 77$

5. The "shtuka" construction $\quad 79$

5.1. The universal shtuka $\quad 79$

5.2. Explicit description of $I$-legged shtukas $\quad 81$

5.3. Partial Frobeniuses 82

5.4. Description of the action via excursions

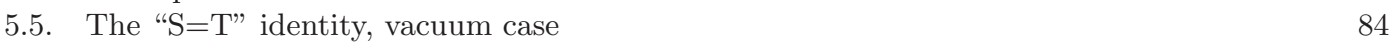

5.6. The " $\mathrm{S}=\mathrm{T}$ " identity, general case $\quad 85$

Appendix A. Sheaves and singular support 87

A.1. Sheaf-theoretic contexts $\quad 87$

A.2. Sheaves on a product $\quad 90$

A.3. Singular support $\quad 92$

A.4. Proof of Theorem A.3.9 in case (a) 94

A.5. Proof of Theorem A.3.9 in the ind-constructible contexts 97

Appendix B. Spectral action in the context of Geometric Langlands (after [NY])

B.1. The players $\quad 100$

B.2. The Hecke action $r \mid 101$

B.3. Digression: naive geometric Satake $\quad 103$

B.4. Hecke action on the subcategory with nilpotent singular support $\quad 104$

\begin{tabular}{lll} 
B.5. & Proof of Theorem B.4.2 & 105 \\
\hline
\end{tabular}

B.6. Preservation of nilpotence of singular support $\quad 106$

Appendix C. Integrated actions in the context of D-modules 111

\begin{tabular}{llr|} 
C.1. Definition of integrated action & 111 \\
\hline
\end{tabular}

\begin{tabular}{ll} 
C.2. Lisse actions & 112 \\
\hline
\end{tabular}

\begin{tabular}{llr|} 
C.3. A spectral characterization of lisse actions & 114 \\
\hline
\end{tabular}

Appendix D. The notion of universal local acyclicity (ULA) $\quad 116$

\begin{tabular}{lll} 
D.1. The abstract ULA property & 116 \\
\hline \hline
\end{tabular}

\begin{tabular}{lll} 
D.2. Adding self-duality & 117 \\
\hline
\end{tabular}

D.3. The ULA condition in the geometric situation $\quad 119$

D.4. An aside: the notion of ULA in other sheaf-theoretic contexts $\quad 120$

\begin{tabular}{ll} 
D.5. The ULA property for D-modules & 123 \\
\hline
\end{tabular}

\begin{tabular}{ll} 
References & 127 \\
\hline
\end{tabular}

\section{INTRODUCTION}

Our goal is to provide a categorical framework that leads to the definition of shtukas à la Drinfeld and of excursion operators à la V. Lafforgue. We will capture the main ingredients of V. Lafforgue's construction, which are:

- The action of the algebra of functions on the stack of arithmetic local systems on the space of automorphic functions;

-The "S=T" identity. 
However, all of this will be performed in a toy setting: the key technical(?) difficulty in V. Lafforgue's work is that the sheaf-theoretic context he needs is that of $\ell$-adic sheaves on schemes over $\mathbb{F}_{q}$. By contrast, we will work in the topological context in the spirit of BN1.

The $\ell$-adic context, which gives rise to actual shtukas, will be considered in subsequent work.

0.1. Hecke action. The point of view taken in this paper is that the geometric ingredient that gives rise to the Drinfeld-Lafforgue construction is the categorical Hecke action. In this subsection we will specify what we mean by this.

0.1.1. We will consider the following three geometric/sheaf-theoretic contexts:

- $\ell$-adic sheaves on schemes over any ground field $k$;

-Sheaves (in the classical topology) with coefficients in a commutative ring e on schemes over $\mathbb{C}$;

-D-modules on schemes over a ground field $k$ of characteristic 0 .

For a scheme/stack $y$, let $\operatorname{Shv}(y)$ denote the corresponding category of sheaves; this is a DG category over our field of coefficients (i.e., over $\mathbb{Q}_{\ell}$, e and $k$, respectively).

0.1.2. Let $X$ be an algebraic curve and $G$ a reductive group (over our ground field). Let Bun denote $^{-}$ the stack of principal $G$-bundles on $X$.

Let $\breve{G}$ be the Langlands dual group of $G$, which is a reductive group over our ring of coefficients.

The point of departure is the Hecke action on $\operatorname{Shv}\left(\operatorname{Bun}_{G}\right)$ of the symmetric monoidal category $\operatorname{Rep}(\check{G})$ integrated over $X$. This is not a completely straightforward notion, and we refer the reader to Sect. 0.2 .1 below or Sect. 1.7 .3 for a detailed discussion.

Here is what this action gives us.

0.1.3. In the context of D-modules, (the rather non-trivial) result of V. Drinfeld and the first-named author (recorded in Ga1, Corollary 4.5.5]) says that this action gives rise to an action of the category

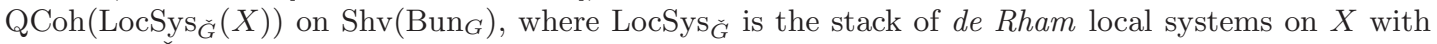
respect to $\check{G}$.

0.1.4. In the context of sheaves on the classical topology, Theorem 6.3 .5 of [NY] says that this action gives rise to an action of $\mathrm{QCoh}\left(\operatorname{LocSys}_{\breve{G}}(X)\right)$ on $\operatorname{Shv}_{\text {Nilp }}\left(\operatorname{Bun}_{G}\right)$, where:

$-\operatorname{LocSys}_{\check{G}}(X)$ is the stack of Betti local systems on $X$ with respect to $\check{G}$;

$-\operatorname{Shv}_{\text {Nilp }}\left(\operatorname{Bun}_{G}\right) \subset \operatorname{Shv}\left(\operatorname{Bun}_{G}\right)$ is the full subcategory consisting of sheaves with nilpotent singular support.

For the reader's convenience, we will review the construction of this action in Sect. B

We should remark that unlike the D-module context, the action of the Betti QCoh( $\left.\operatorname{LocSys}_{\breve{G}}(X)\right)$ on $\operatorname{Shv}_{\mathrm{Nilp}}\left(\operatorname{Bun}_{G}\right)$ is obtained relatively easily from what we state in the present paper as Theorem 1.5.5 (which in itself is not a difficult assertion either), combined with the key observation of [NY] Theorem 6.1.1] about the behavior of singular support.

0.1.5. Our main interest, however, is when the sheaf-theoretic context is that of $\ell$-adic sheaves. The first conceptual difficulty in this case is that we do not have a direct analog of $\operatorname{LocSys}_{\breve{G}}(X)$ as an algebrogeometric object (over $\left.\mathbb{Q}_{l}\right)$, so we cannot talk about an action of $\operatorname{QCoh}\left(\operatorname{LocSys}_{\breve{G}}(X)\right)$ on $\operatorname{Shv}\left(\operatorname{Bun}_{G}\right)$.

Nevertheless, the situation is not as hopeless as it might seem, and we will discuss it in detail in a subsequent publication.

0.2. What is done in this paper? We will now outline the actual mathematical contents of the present paper. Each of the steps we perform is a toy (more precisely, Betti) analog of what one wishes to be able to do in the context of $\ell$-adic sheaves. 
0.2.1. Let $\mathcal{A}$ be a symmetric monoidal DG category, and let $Y$ be a space, i.e., $Y$ is an object of the category Spc, see Sect.0.4.1 (we can also think of $Y$ as a homotopy type). To this data one can associate a new symmetric monoidal category $\mathcal{A}^{\otimes Y}$, see Sect. 1.2. Sometimes one also uses the notation 1

$$
\int_{Y} \mathcal{A}:=\mathcal{A}^{\otimes Y} .
$$

For example, if $Y$ is a finite set $I$, we have

$$
\mathcal{A}^{\otimes Y}=\mathcal{A}^{\otimes I},
$$

i.e., the usual $I$-fold tensor product of copies of $\mathcal{A}$. For a general $Y$, the construction can be described by a colimit procedure off the finite set case.

For example, we show (see Theorem 1.5.5) that for $\mathcal{A}=\operatorname{Rep}(\mathrm{G})$ (here $\mathrm{G}$ is an algebraic group), under some condition: 2 , we have a canonical equivalence

$$
\operatorname{Rep}(\mathrm{G})^{\otimes Y} \simeq \mathrm{QCoh}\left(\operatorname{LocSys}_{\mathrm{G}}(Y)\right)
$$

where $\operatorname{LocSys}_{G}(Y)$ is the (derived) stack classifying $G$-local systems on $Y$, i.e., its value on a test affine derived scheme $S$ is the space of symmetric monoidal right t-exact functors $\operatorname{Rep}(\mathrm{G}) \rightarrow \mathrm{QCoh}(S)$, parameterized by $Y$.

Our point of departure is a DG category $\mathcal{M}$, equipped with an action of $\mathcal{A}^{\otimes Y}$ as a monoidal category. We emphasize that this is not the same as a family of monoidal actions of $\mathcal{A}$ on $\mathcal{M}$, parameterized by $Y$, see Remark 1.7.4

We give an explicit description of what the datum of such an action amounts to (see Proposition 1.7.2). Namely, it is equivalent to a compatible family of actions, one for each finite set $I$, of $\mathcal{A}^{\otimes I}$ on $\mathcal{M}$, parameterized by points of $Y^{I}$. This description is useful, because the Hecke action of $\operatorname{Rep}(\check{G})$ on $\operatorname{Shv}\left(\operatorname{Bun}_{G}\right)$ is given in exactly such form, see Sect. B.2

Similarly, we show that the datum of a functor from $\mathcal{A}^{\otimes Y}$ to some DG category $\mathcal{C}$ is equivalent to a compatible family of functors, one for each finite set $I$,

$$
\mathcal{S}_{I}: Y^{I} \times \mathcal{A}^{\otimes I} \rightarrow \mathcal{C} .
$$

This description is useful as it will explain the connection between the universal shtuka and $I$-legged shtukas, see Sect. 5.2

0.2.2. Next, we provide the general framework for excursion operators. Let $\mathcal{A}$ and $Y$ be as before, and let

$$
\mathcal{S}_{Y}: \mathcal{A}^{\otimes Y} \rightarrow \mathcal{C}
$$

be a functor of DG categories.

Consider the object

$$
\mathcal{S}_{Y}\left(\mathbf{1}_{\mathcal{A} \otimes Y}\right) \in \mathcal{C} .
$$

It carries an action of the (commutative) algebra $\mathcal{E} n d_{\mathcal{A} \otimes Y}\left(\mathbf{1}_{\mathcal{A} \otimes Y}\right)$. We give an explicit description of this action, in the spirit of [Laf, Sect. 9].

First, we give an explicit description of the algebra $\mathcal{E} n d_{\mathcal{A} \otimes Y}\left(\mathbf{1}_{\mathcal{A} \otimes Y}\right)$ as a colimit, in the case when $Y$ is connected, and $\mathcal{A}$ has an affine diagonal, see Corollary 2.4.4. Choose a base point $y \in Y$. The index category in the colimit in question is that of pairs $\left(I, \gamma^{I}\right)$, where $I$ is a finite set, and $\gamma^{I}$ is an $I$-tuple of loops in $Y$ based at $y$. (Note that this category is sifted, so the colimit in the category of commutative algebras is the same as the colimit of underlying associative algebras and is also the same as the colimit of the underlying vector spaces.) The terms of the colimit are described as follows. The term corresponding to a finite set $I$ is given by

$$
\mathcal{H}_{\operatorname{com}}\left(\mathbf{1}_{\mathcal{A}}, \operatorname{mult}_{I_{+}} \circ \operatorname{mult}_{I_{+}}^{R}\left(\mathbf{1}_{\mathcal{A}}\right)\right),
$$

where:

\footnotetext{
${ }^{1}$ Yet another name for $\mathcal{A}^{\otimes Y}$ is "chiral (or factorization) homology of $\mathcal{A}$ along $Y$ ".

${ }^{2}$ Specifically, if $Y$ has finitely many connected components and the ring of coefficients e contains $\mathbb{Q}$.
} 
$-I_{+}=I \sqcup\{*\} ;$

-For a finite set $J$, we denote by mult $J$ the tensor product map $\mathcal{A}^{\otimes J} \rightarrow \mathcal{A}$;

- mult $_{J}^{R}$ denotes the right adjoint functor of mult ${ }_{J}$.

The algebra structure on (0.2) comes from the right-lax symmetric monoidal structure on mult ${ }_{J}^{R}$, obtained by adjunction from the symmetric monoidal structure on mult $J$.

0.2.3. Next, given a functor $\mathcal{S}_{Y}: \mathcal{A}^{\otimes Y} \rightarrow \mathcal{C}$, we show how each term (0.2) acts on $\mathcal{S}_{Y}\left(\mathbf{1}_{\mathcal{A} \otimes Y}\right)$, see Theorem 2.8.7

Namely, given $\xi \in \mathcal{H}^{\circ} m_{\mathcal{A}}\left(\mathbf{1}_{\mathcal{A}}, \operatorname{mult}_{I_{+}} \circ \operatorname{mult}_{I_{+}}^{R}\left(\mathbf{1}_{\mathcal{A}}\right)\right)$, the corresponding endomorphism of $\mathcal{S}_{Y}\left(\mathbf{1}_{\mathcal{A} \otimes Y}\right)$ is the excursion operator:

$$
\begin{array}{r}
\mathcal{S}_{Y}\left(\mathbf{1}_{\mathcal{A} \otimes Y}\right) \longrightarrow \mathcal{S}_{\{*\}}\left(y, \mathbf{1}_{\mathcal{A}}\right) \stackrel{\xi}{\perp} \mathcal{S}_{\{*\}}\left(y, \operatorname{mult}_{I_{+}} \circ\left(\operatorname{mult}_{I_{+}}\right)^{R}\left(\mathbf{1}_{\mathcal{A}}\right)\right) \\
\mathcal{S}_{I_{+}}\left(y^{I_{+}},\left(\operatorname{mult}_{I_{+}}\right)^{R}\left(\mathbf{1}_{\mathcal{A}}\right)\right) \\
\downarrow \text { mon }_{\gamma^{I_{+}}} \\
\mathcal{S}_{I_{+}}\left(y^{I_{+}},\left(\operatorname{mult}_{I_{+}}\right)^{R}\left(\mathbf{1}_{\mathcal{A}}\right)\right) \\
\downarrow \\
\mathcal{S}_{Y}\left(\mathbf{1}_{\mathcal{A} \otimes Y}\right) \stackrel{\sim}{\sim} \mathcal{S}_{\{*\}}\left(y, \mathbf{1}_{\mathcal{A}}\right) \stackrel{\text { counit }}{\longleftarrow} \mathcal{S}_{\{*\}}\left(y, \operatorname{mult}_{I_{+}} \circ\left(\operatorname{mult}_{I_{+}}\right)^{R}\left(\mathbf{1}_{\mathcal{A}}\right)\right),
\end{array}
$$

where

$-\gamma^{I+}=\left(\gamma^{I}, \gamma_{\text {triv }}\right)$

-For a finite set $J$, and a $J$-tuple $\gamma^{J}$ of loops in $Y$ based at $y$, viewed as a loop into $Y^{J}$ based at $y^{J}$, we denote by $\operatorname{mon}_{\gamma^{J}}$ the corresponding automorphism of $\mathcal{S}_{J}\left(y^{J},-\right)$.

In the particular case of $\mathcal{A}=\operatorname{Rep}(\mathrm{G})$, from the colimit expression of Sect. 0.2.2 and 0.1 , we obtain an explicit description of the algebra

$$
\Gamma\left(\operatorname{LocSys}_{\mathrm{G}}(Y), \mathcal{O}_{\operatorname{LocSys}_{G}(Y)}\right)
$$

in terms excursion operators. This recovers the analogs of the formulas from Laf Sect. 10 and Proposition 11.7].

0.2.4. We now come to the next main ingredient of this paper, namely the notion of categorical trace. First, we recall that given a symmetric monoidal category $\mathbf{O}$ and a dualizable object $\mathbf{O} \in \mathbf{O}$ equipped with an endomorphism $F$, we can assign to this data a point

$$
\operatorname{Tr}(F, \mathbf{o}) \in \operatorname{Endo}_{\mathbf{O}}\left(\mathbf{1}_{\mathbf{O}}\right),
$$

called the trace of $F$ on o, see Sect. 3.1.1

Suppose now that $\mathbf{O}$ is actually a symmetric monoidal 2-category (i.e., we have not necessarily invertible 2-morphisms). Let us be given a pair of dualizable objects $\mathbf{o}_{1}, \mathbf{o}_{2} \in \mathbf{O}$, each equipped with an endomorphism $F_{i}, i=1,2$. Assume in addition that we are given a 1-morphism $t: \mathbf{o}_{1} \rightarrow \mathbf{o}_{2}$ that admits a right adjoint. Finally, suppose that $t$ intertwines $F_{1}$ and $F_{2}$, up to a not necessarily invertible 2-morphism $\alpha$, i.e.,

$$
t \circ F_{1} \stackrel{\alpha}{\rightarrow} F_{2} \circ t .
$$

We show that to this data there corresponds a 2-morphism

$$
\operatorname{Tr}(t, \alpha): \operatorname{Tr}\left(F_{1}, \mathbf{o}_{1}\right) \rightarrow \operatorname{Tr}\left(F_{2}, \mathbf{o}_{2}\right) .
$$

In fact, the 2-morphism $\operatorname{Tr}(t, \alpha)$ can be explicitly described as

$$
\operatorname{Tr}\left(F_{1}, \mathbf{o}_{1}\right) \rightarrow \operatorname{Tr}\left(t^{R} \circ t \circ F_{1}, \mathbf{o}_{1}\right) \simeq \operatorname{Tr}\left(t \circ F_{1} \circ t^{R}, \mathbf{o}_{2}\right) \rightarrow \operatorname{Tr}\left(F_{2} \circ t \circ t^{R}, \mathbf{o}_{2}\right) \rightarrow \operatorname{Tr}\left(F_{2}, \mathbf{o}_{2}\right),
$$

where: 
$-t^{R}$ denotes the right adjoint of $t$;

-The first arrow is induced by the unit of the adjunction $\operatorname{id}_{\mathbf{o}_{1}} \rightarrow t^{R} \circ t$;

-The second arrow is given by the cyclicity property of trace;

-The third arrow is induced by $\alpha$;

- The fourth arrow is induced by the counit of the adjunction $t \circ t^{R} \rightarrow \operatorname{id}_{\mathbf{o}_{2}}$.

0.2.5. We apply the above formalism in the following two main examples: $\mathbf{O}=$ DGCat and $\mathbf{O}=$ Morita(DGCat) (see right below for the definition of the latter).

In the case of $\mathbf{O}=$ DGCat, to a (dualizable) DG category $\mathcal{C}$ equipped with an endofunctor $F$, we attach

$$
\operatorname{Tr}(F, \mathcal{C}) \in \text { Vect, }
$$

and to a pair of such, equipped with a functor $T: \mathcal{C}_{1} \rightarrow \mathcal{C}_{2}$ (that admits a continuous right adjoint) and a natural transformation

$$
\alpha: T \circ F_{1} \rightarrow F_{2} \circ T
$$

we attach a map in Vect

$$
\operatorname{Tr}(T, \alpha): \operatorname{Tr}\left(F_{1}, \mathrm{C}_{1}\right) \rightarrow \operatorname{Tr}\left(F_{2}, \mathrm{C}_{2}\right) .
$$

0.2.6. The case of $\mathbf{O}=$ Morita(DGCat) is obviously richer. By definition, the objects of $\mathbf{O}=$ Morita(DGCat) are 2-DG categories of the form $\mathcal{R}$ - mod, where $\mathcal{R}$ is a monoidal DG category. Further, 1-morphisms in Morita(DGCat) are by definition given by bi-module categories 3 .

To a 2-DG category $\mathfrak{C}$ equipped with an endofunctor $\mathfrak{F}$ we now attach a $D G$ category $\operatorname{Tr}(\mathfrak{F}, \mathfrak{C})$. To a pair of such, equipped with a functor $\mathfrak{T}: \mathfrak{C}_{1} \rightarrow \mathfrak{C}_{2}$ (that admits a right adjoint in Morita(DGCat)) and a natural transformation

$$
\alpha: \mathfrak{T} \circ \mathfrak{F}_{1} \rightarrow \mathfrak{F}_{2} \circ \mathfrak{T},
$$

we attach a functor between DG categories

$$
\operatorname{Tr}(\mathfrak{T}, \alpha): \operatorname{Tr}\left(\mathfrak{F}_{1}, \mathfrak{C}_{1}\right) \rightarrow \operatorname{Tr}\left(\mathfrak{F}_{2}, \mathfrak{C}_{2}\right) .
$$

For $\mathfrak{C}=\mathcal{R}-\bmod$ and $\mathfrak{F}$ given by

$$
Q \in\left(\mathcal{R} \otimes \mathcal{R}^{\text {rev }}\right) \text { - mod, }
$$

the resulting DG category $\operatorname{Tr}(\mathfrak{F}, \mathfrak{C})$ identifies with

$$
\mathrm{HH}_{\bullet}(\mathcal{R}, \mathcal{Q}):=\mathcal{R} \underset{\mathcal{R} \otimes \mathcal{R}^{\mathrm{rev}}}{\otimes} Q,
$$

this is the category of Hochschild chains on $\mathcal{R}$ with coefficients in $\mathcal{Q}$.

0.2.7. In Theorem 3.8.5 we establish a basic compatibility between the categorical and the 2-categorical trace constructions:

Namely, let $\mathcal{R}$ be a symmetric monoidal DG category; assume that $\mathcal{R}$ is rigid. Let $\mathcal{M}$ be a module category over $\mathcal{R}$. We can view $\mathcal{M}$ as a 1 -morphism

$$
\text { DGCat } \rightarrow \mathcal{R} \text { - mod. }
$$

Assume that $\mathcal{M}$ is dualizable as a DG category. This condition is equivalent requiring that the above 1-morphism admit a right adjoint.

Let $F_{\mathcal{R}}$ be a symmetric monoidal endofunctor of $\mathcal{R}$. Let $F_{\mathcal{M}}$ be an endofunctor of $\mathcal{M}$, endowed with a datum of compatibility with $F_{\mathcal{R}}$; i.e., we have a datum of commutativity of the diagram

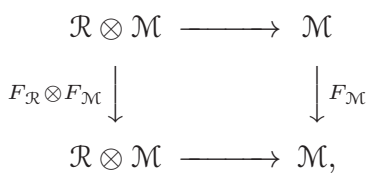

${ }^{3}$ We note that every object of Morita(DGCat) is dualizable: the dual of $\mathcal{R}-\bmod$ is $\mathcal{R}^{\text {rev }}-\mathbf{m o d}$, where $\mathcal{R}^{\text {rev }}$ is obtained from $\mathcal{R}$ by reversing the monoidal structure. 
along with higher compatibilities. We can view this compatibility structure as a data of " $\alpha$ " for the 1-morphism (0.6). Hence, by (0.5), to this data there corresponds a map in DGCat

$$
\text { Vect } \rightarrow \operatorname{Tr}\left(F_{\mathcal{R}}, \mathcal{R} \text { - mod }\right) .
$$

In other words, we obtain an object

$$
\operatorname{cl}\left(\mathcal{M}, F_{\mathcal{M}}\right) \in \operatorname{Tr}\left(F_{\mathcal{R}}, \mathcal{R}-\mathbf{m o d}\right) \simeq \mathrm{HH}_{\bullet}\left(\mathcal{R}, F_{\mathcal{R}}\right) .
$$

Note that since $\mathcal{R}$ was symmetric monoidal, and $F_{\mathcal{R}}$ was also symmetric monoidal, the category $\mathrm{HH}_{\bullet}\left(\mathcal{R}, F_{\mathcal{R}}\right)$ acquires a symmetric monoidal structure. Let $\mathbf{1}_{\mathrm{HH}}\left(\mathcal{R}, F_{\mathcal{R}}\right)$ denote the unit object in $\mathrm{HH}_{\bullet}\left(\mathcal{R}, F_{\mathcal{R}}\right)$.

Our Theorem 3.8 .5 says that there exists a canonical isomorphism

$$
\mathcal{H}^{\circ} m_{\mathrm{HH}}\left(\mathcal{R}, F_{\mathcal{R}}\right)\left(\mathbf{1}_{\mathrm{HH} \cdot\left(\mathcal{R}, F_{\mathcal{R}}\right)}, \operatorname{cl}\left(\mathcal{M}, F_{\mathcal{M}}\right)\right) \simeq \operatorname{Tr}\left(F_{\mathcal{M}}, \mathcal{M}\right) .
$$

Isomorphism (0.7) shows that $\operatorname{cl}\left(\mathcal{M}, F_{\mathcal{M}}\right)$ lifts $\operatorname{Tr}\left(F_{\mathcal{M}}, \mathcal{M}\right)$ to an object of $\mathrm{HH}_{\bullet}\left(\mathcal{R}, F_{\mathcal{R}}\right)$; this justifies the notation:

So Theorem 3.8.5 says that

$$
\operatorname{Tr}_{\mathcal{R}}^{\mathrm{enh}}\left(F_{\mathcal{M}}, \mathcal{M}\right):=\operatorname{cl}\left(\mathcal{M}, F_{\mathcal{M}}\right) .
$$

$$
\mathcal{H}_{o m_{\mathrm{HH}}\left(\mathcal{R}, F_{\mathcal{R}}\right)}\left(\mathbf{1}_{\mathrm{HH} \bullet\left(\mathcal{R}, F_{\mathcal{R}}\right)}, \operatorname{Tr}_{\mathcal{R}}^{\mathrm{enh}}\left(F_{\mathcal{M}}, \mathcal{M}\right)\right) \simeq \operatorname{Tr}\left(F_{\mathcal{M}}, \mathcal{M}\right)
$$

0.2.8. We now come to the central construction in this paper, which is the prototype of the shtuka construction.

Let us be given a symmetric monoidal category $\mathcal{A}$ and a space $Y$. Let now $\phi$ be an endomorphism of $Y$, which induces a symmetric monoidal endofunctor, denoted $\mathcal{A}^{\otimes \phi}$, of $\mathcal{A}^{\otimes Y}$. Let $Y / \phi$ be the (homotopy) quotient of $Y$ by $\phi$.

First, we note that there exists a canonical equivalence

$$
\mathrm{HH}_{\bullet}\left(\mathcal{A}^{\otimes Y}, \mathcal{A}^{\otimes \phi}\right) \simeq \mathcal{A}^{\otimes Y / \phi} .
$$

Next, let us be given an action of $\mathcal{A}^{\otimes Y}$ on a (dualizable) DG category $\mathcal{M}$. Let $F_{\mathcal{M}}$ be an endofunctor of $\mathcal{M}$ compatible with $\mathcal{A}^{\otimes \phi}$.

Let

$$
\operatorname{Sht}_{\mathcal{M}, F_{\mathcal{M}}, \text { univ }} \in \mathcal{A}^{\otimes Y / \phi}
$$

be the object that corresponds under (0.8) to

$$
\operatorname{Tr}_{\mathcal{A} \otimes Y}^{\mathrm{enh}}\left(F_{\mathcal{M}}, \mathcal{M}\right) \in \mathrm{HH}_{\bullet}\left(\mathcal{A}^{\otimes Y}, \mathcal{A}^{\otimes \phi}\right) .
$$

0.2.9. In Sect. 5 we show how the object $\operatorname{Sht}_{\mathcal{M}, F_{\mathcal{M}} \text {, univ }}$ encodes (i) the $I$-legged shtuka construction 4 , (ii) partial Frobeniuses, (iii) excursion operators, and (iv) the " $\mathrm{S}=\mathrm{T}$ " relation. In more detail:

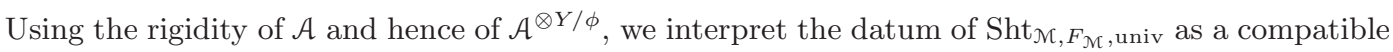
family of functors

$$
\operatorname{Sht}_{\mathcal{M}, F_{\mathcal{M}}, Y / \phi, I}:(Y / \phi)^{I} \times \mathcal{A}^{\otimes I} \rightarrow \text { Vect }
$$

Let

$$
\operatorname{Sht}_{\mathcal{M}, F_{\mathcal{M}}, Y, I}: Y^{I} \times \mathcal{A}^{\otimes I} \rightarrow \text { Vect }
$$

be the precomposition of $\operatorname{Sht}_{\mathcal{M}, F_{\mathcal{M}}, Y / \phi, I}$ with the tautological projection $Y^{I} \rightarrow(Y / \phi)^{I}$.

(i) In Proposition 5.2.4 we show that the value of the functor Sht $\mathcal{M}_{\mathcal{M}, F_{\mathcal{M}}, Y, I}$ on

$$
\underline{y} \in Y^{I}, r \in \mathcal{A}^{\otimes I}
$$

identifies with

$$
\operatorname{Tr}\left(H_{r_{\underline{y}}} \circ F_{\mathcal{M}}, \mathcal{M}\right)
$$

where:

\footnotetext{
${ }^{4}$ For us shtukas are algebraic objects. What we call "shtukas" is more commonly called the "cohomology of sheaves arising by geometric Satake on (geometric) shtukas". See Sect. 3.5.11 for an explanation of why this corresponds to cohomology of sheaves on the (geometric) moduli space of shtukas.
} 
${ }^{-} r_{\underline{y}}$ denotes the object of $\mathcal{A}^{\otimes Y}$ equal to the image of $r$ under $\mathcal{A}^{\otimes I} \stackrel{\underline{y}}{\rightarrow} \mathcal{A}^{\otimes Y}$;

-For $r^{\prime} \in \mathcal{A}^{\otimes Y}$, we let $H_{r^{\prime}}$ denotes the endofunctor of $\mathcal{M}$ given by the action of $r^{\prime}$.

This should be seen as a direct analog of the $I$-legged shtuka construction.

(ii) The fact that $\operatorname{Sht}_{\mathcal{M}, F_{\mathcal{M}}, Y, I}$ comes from $\operatorname{Sht}_{\mathcal{M}, F_{\mathcal{M}}, Y / \phi, I}$ means that the former is equivariant under each of the endomorphisms $\phi_{i}$ of $Y^{I}$, that acts as $\phi$ along the factor of $Y^{I}$ corresponding to $i \in I$ and as the identity along the other factors. Following V. Drinfeld, L. Lafforgue and V. Lafforgue, we call these endomorphisms "partial Frobeniuses". We show (see Proposition 5.3.3) that, in terms of the isomorphism

$$
\operatorname{Sht}_{\mathcal{M}, F_{\mathcal{M}}, Y, I}\left(\underline{y} \in Y^{I}, r \in \mathcal{A}^{\otimes I}\right) \simeq \operatorname{Tr}\left(H_{r_{\underline{y}}} \circ F_{\mathcal{X}}, \mathcal{M}\right),
$$

the formula for the action of partial Frobeniuses translates to the construction from [Laf, Sect. 3]. (We note that a salient feature of this formula is cyclicity property of the trace construction.)

(iii) By Theorem 3.8.5 mentioned above, we have a canonical isomorphism

$$
\operatorname{Tr}\left(F_{\mathcal{M}}, \mathcal{M}\right) \simeq \mathcal{H}_{\mathcal{A}} m_{\mathcal{A} \otimes Y / \phi}\left(\mathbf{1}_{\mathcal{A} \otimes Y / \phi}, \operatorname{Sht}_{\mathcal{M}, F_{\mathcal{M}}, \text { univ }}\right) \simeq \operatorname{Sht}_{\mathcal{M}, F_{\mathcal{M}}, Y / \phi, \emptyset}
$$

In particular, $\operatorname{Tr}\left(F_{\mathcal{M}}, \mathcal{M}\right)$ acquires an action of the algebra $\mathcal{E} n d_{\mathcal{A} \otimes Y / \phi}\left(\mathbf{1}_{\mathcal{A} \otimes Y / \phi}\right)$. In Proposition 5.4.3 we describe this action explicitly in terms of the excursion operators, which are direct analogs of those in [Laf, Sect. 9].

(iv) Arguably, the key conceptual and computational place in V. Lafforgue's paper is the " $\mathrm{S}=\mathrm{T}$ " relation, which appears as Laf Proposition 6.2]. In Theorem 5.5.5 we state and prove an analog of this result in our abstract context. It states the equality of two particular endomorphisms (one called $S$ and the other $T)$ of $\operatorname{Tr}\left(F_{\mathcal{M}}, \mathcal{M}\right)$ corresponding to the data of $\left(y_{0}, a\right)$, where $y_{0}$ is a $\phi$-fixed point on $Y$, and $a$ is a compact object in $\mathcal{A}$.

The $T$ operator is given by (0.4), for the endofunctor $H_{a_{y_{0}}} \circ F_{\mathcal{M}}$ of $\mathcal{M}$.

The $S$ operator is an explicit excursion operator corresponding to $I=\{*\}$, the tautological loop based at the image $\bar{y}_{0}$ of $y_{0}$ under $Y \rightarrow Y / \phi$ (here we use the fact that $y_{0}$ is fixed by $\phi$ ), and a canonical map

$$
\xi_{a}: \mathbf{1}_{\mathcal{A}} \rightarrow \text { mult } \circ \text { mult }^{R}\left(\mathbf{1}_{\mathcal{A}}\right)
$$

attached to $a$, see 4.14).

0.3. Organization of the paper. We will now briefly describe the structure of the paper.

0.3.1. In Sect. 1 we study the operation

$$
Y, \mathcal{A} \mapsto \mathcal{A}^{\otimes Y},
$$

where $Y$ is a space and $\mathcal{A}$ is a symmetric monoidal category.

The main results of this section are:

-Presentation of $\mathcal{A}^{\otimes Y}$ as a colimit with terms $\mathcal{A}^{\otimes I}$ for finite sets $I$, as a symmetric monoidal category/monoidal category/DG category (Theorem 1.2.4);

-Description of functors $\mathcal{A}^{\otimes Y} \rightarrow \mathcal{C}$ as compatible families of functors $\mathcal{A}^{\otimes I} \rightarrow \mathcal{C} \otimes \operatorname{LS}\left(Y^{I}\right)$, where $\operatorname{LS}(-)$ denotes the category of (topological) local systems on a given space (Proposition 1.7.2);

-For a group G and a space $Y$ (with finitely many connected components), an equivalence

$$
\operatorname{Rep}(\mathrm{G})^{\otimes Y} \rightarrow \operatorname{QCoh}\left(\operatorname{LocSys}_{\mathrm{G}}(Y)\right),
$$

where $\operatorname{LocSys}_{\mathrm{G}}(Y)$ is the derived algebraic stack, classifying (topological) G-local systems on $Y$ (Theorem 1.5.5), and a generalization of this statement when $\operatorname{Rep}(\mathrm{G})=\mathrm{QCoh}(\mathrm{pt} / \mathrm{G})$ is replaced by $\mathrm{QCoh}$ of a more general algebraic stack. 
0.3.2. The main theme of Sect. 2 is excursion operators. The main results of this section are:

-In the case when $Y$ is a connected pointed space, a presentation of $\mathcal{A}^{\otimes Y}$ as a (sifted) colimit with terms

$$
\mathcal{A}^{\otimes \Sigma\left(I_{+}\right)},
$$

where $I_{+}=I \sqcup *$ is a pointed finite set, and $\Sigma\left(I_{+}\right) \in$ Spc denotes the suspension of $I_{+}$, i.e., $\Sigma\left(I_{+}\right) \simeq \underset{I}{\vee} S^{1}$ (Corollary 2.1.6);

-Assuming that $\mathcal{A}$ has an affine diagonal, a description of $\mathcal{E} n d_{\mathcal{A} \otimes Y}\left(\mathbf{1}_{\mathcal{A} \otimes Y}\right)$ as a colimit with terms $\varepsilon n d_{\mathcal{A}^{\otimes \Sigma\left(I_{+}\right)}}\left(\mathbf{1}_{\mathcal{A}}^{\otimes \Sigma\left(I_{+}\right)}\right)$(Corollary 2.4.4);

-For $\mathcal{A}$ rigid, an identification of $\varepsilon n d_{\mathcal{A} \otimes \Sigma(J)}\left(\mathbf{1}_{\mathcal{A} \otimes \Sigma(J)}\right)$ with $\mathcal{H}_{\text {om }} \boldsymbol{A}_{\mathcal{A}}\left(\mathbf{1}_{\mathcal{A}}\right.$, mult $_{J} \circ$ mult $_{J}^{R}\left(\mathbf{1}_{\mathcal{A}}\right)$ ) (Corollary 2.7.6);

-For a functor $\mathcal{S}_{Y}: \mathcal{A}^{\otimes Y} \rightarrow \mathcal{C}$, an expression for the action of the terms

$$
\mathcal{H}_{\operatorname{orm}}\left(\mathbf{1}_{\mathcal{A}}, \operatorname{mult}_{J} \circ \operatorname{mult}_{J}^{R}\left(\mathbf{1}_{\mathcal{A}}\right)\right) \rightarrow \mathcal{E} n d_{\mathcal{A} \otimes \Sigma(J)}\left(\mathbf{1}_{\mathcal{A} \otimes \Sigma(J)}\right) \rightarrow \mathcal{E} n d_{\mathcal{A} \otimes Y}\left(\mathbf{1}_{\mathcal{A} \otimes Y}\right)
$$

on $\mathcal{S}_{Y}\left(\mathbf{1}_{\mathcal{A} \otimes Y}\right)$ in terms of the excursion operators (Theorem 2.8.7).

0.3.3. In Sect. 3 we study the operation of categorical trace. The main results of this section are:

-Construction of the categorical trace (Sect. 3.2);

-Construction of the 2-categorical trace (Sect. 3.6);

-The relationship between the two for rigid symmetric monoidal categories (Theorem 3.8.5).

0.3.4. In Sect. 4 we study several generalizations and elaborations of Theorem 3.8.5 The main results are:

-We connect the Chern character of a compact object of a rigid symmetric monoidal category with the excursion operator (Corollary 4.3.6);

-We formulate and prove a version of Theorem 3.8.5 "with observables" (Theorem 4.4.4);

-We introduce a mechanism that stands behind the Drinfeld-Lafforgue-Lafforgue definition of partial Frobeniuses (Lemma 4.5.4).

0.3.5. In Sect. 5 we combine the material of the previous sections to obtain our toy model for the shtuka construction. The material in this section has been described already in Sect. 0.2.9

0.3.6. In the main body of this paper we study actions of categories of the form $\mathcal{A}^{\otimes Y}$, where $\mathcal{A}$ is a symmetric monoidal category, and $Y$ is an object of Spc. A key example is furnished by [NY]: when working over the ground field $\mathbb{C}$, we have an action of $\operatorname{Rep}(\check{G})^{\otimes X^{\text {top sing }}}$ on $\operatorname{Shv}_{\text {Nilp }}\left(\operatorname{Bun}_{G}\right)$, where $X^{\text {top,alg }}$ is the object of Spc corresponding to our curve $X$, and $\operatorname{Shv}(-)$ denotes the category of sheaves in the classical topology. (We recall that according to Theorem 1.5.5 the category $\operatorname{Rep}(\check{G})^{\otimes X^{\text {top }, \text { sing }}}$ identifies with QCoh( $\left.\operatorname{LocSys}_{\check{G}, \text { Betti }}\right)$.)

In the Appendix, Sects. A.D we investigate the possibility of extending the construction of [NY] to other sheaf-theoretic contexts. 
0.3.7. In Sect. A we introduce a list of sheaf-theoretic contexts that we will consider. This list includes sheaves in the classical topology (for schemes/stacks over $\mathbb{C}$ ), D-modules (for schemes/stacks over a ground field of characteristic zero), as well as étale sheaves (over any ground field).

We recall the notion of singular support of an object $\mathcal{F} \in \operatorname{Shv}(y)$, where $y$ is a scheme or algebraic stack.

The main result of Sect. A is Theorem A.3.9 which says that given an algebraic stack $y$ and a conical subset $\mathcal{N} \subset T^{*}(y)$, for a scheme $X$, the functor of external tensor product defines an equivalence

$$
\operatorname{Shv\mathcal {N}}_{\mathcal{N}}(y) \otimes \operatorname{Shv}_{\text {lisse }}(X) \rightarrow \operatorname{Shv}_{\mathcal{N}^{\prime}}(y \times X), \quad \mathcal{N}^{\prime}:=\mathcal{N} \times\{\text { zero-section }\},
$$

under the assumption that $X$ is proper 5 . In the above formula $\operatorname{Shv}_{\text {lisse }}(X) \subset \operatorname{Shv}(X)$ is the subcategory of lisse objects, see Sect. A.3.7.

0.3.8. In Sect. B we generalize the construction of [NY] about the action of $\operatorname{Rep}(\check{G})^{\otimes X^{\mathrm{top}, \text { sing }}}$ on $\operatorname{Shv}_{\text {Nilp }}\left(\operatorname{Bun}_{G}\right)$.

First, we provide details for the general pattern of Hecke action: we show that in any sheaf-theoretic context, there is a compatible family of actions of $\operatorname{Rep}(\breve{G})^{\otimes I}$ on $\operatorname{Shv}\left(\operatorname{Bun}_{G} \times X^{I}\right)$ as $I$ ranges over the category of finite sets.

Next, we show that the Hecke action gives rise to a compatible family of monoidal functors

$$
\operatorname{Rep}(\check{G})^{\otimes I} \rightarrow \operatorname{End}\left(\operatorname{Shv}_{\text {Nilp }}\left(\operatorname{Bun}_{G}\right)\right) \otimes \operatorname{Shvlisse}_{\text {li }}(X), \quad I \in \text { fSet } .
$$

Finally, if the sheaf-theoretic context is that of sheaves in the classical topology, we show that the functors (0.9) assemble to an action of $\operatorname{LocSys}_{\tilde{G}}(X)$ on $\operatorname{Shv}_{\mathrm{Nilp}}\left(\operatorname{Bun}_{G}\right)$.

0.3.9. In Sect. C we specialize to the case when our sheaf-theoretic context is that of D-modules. We formulate several conjectures pertaining to integrated actions in this context.

First off, for a symmetric monoidal category $\mathcal{A}$ and a scheme $X$, we define an action of $\mathcal{A}^{\otimes X}$ on a DG category $\mathcal{M}$, to be the same as a compatible family of monoidal functors

$$
\mathcal{A}^{\otimes I} \rightarrow \operatorname{End}(\mathcal{M}) \otimes \mathrm{D}-\bmod \left(X^{I}\right), \quad I \in \mathrm{fSet} .
$$

This is equivalent to having an action on $\mathcal{M}$ of the symmetric monoidal category $\operatorname{Fact}(\mathcal{A})_{\operatorname{Ran}(X)}$, defined as in Ga3, Sect. 2.5].

A key example of such an action is when $\mathcal{M}=\mathrm{D}-\bmod \left(\operatorname{Bun}_{G}\right)$ and $\mathcal{A}=\operatorname{Rep}(\check{G})$.

Let $\operatorname{LocSys}_{\breve{G}}(X)$ be the stack of de Rham $\breve{G}$-local systems on $X$, defined as in [AG, Sect. 10.1]. According to [Ga1 Sect. 4.3], we have a canonically defined symmetric monoidal functor

$$
\left.\operatorname{Rep}(\check{G})^{\otimes X} \rightarrow \mathrm{QCoh} \operatorname{LocSys}_{\breve{G}}(X)\right),
$$

which admits a fully faithful right adjoint. Hence, among categories equipped with an action of $\operatorname{Rep}(\check{G})^{\otimes X}$ there is a full subcategory formed by those categories on which this action comes from an action of $\mathrm{QCoh}\left(\operatorname{LocSys}_{\breve{G}}(X)\right)$.

A result that we mentioned earlier on says that $\mathrm{D}-\bmod \left(\mathrm{Bun}_{G}\right)$ belongs to the above subcategory. However, the proof of this theorem heavily relied on "non-geometric" constructions, specifically on the interaction between $\mathrm{D}-\bmod \left(\operatorname{Bun}_{G}\right)$ and representation theory of the affine Kac-Moody Lie algebra corresponding to $\mathfrak{g}$ at the critical level. Given that, one would have liked to have a more geometric proof of this result.

Unfortunately, we do not have a conjecture as to when an action of $\operatorname{Rep}(\check{G})^{\otimes X}$ factors via $\left.\mathrm{QCoh} \operatorname{LocSys}_{\breve{G}}(X)\right)$.

Next, we introduce what it means for an action of $\mathcal{A}^{\otimes X}$ on $\mathcal{M}$ to be lisse. This means that for any $r \in \mathcal{A}^{\otimes I}$ and $m \in \mathcal{M}$, the resulting object of $\mathcal{M} \otimes \mathrm{D}-\bmod \left(X^{I}\right)$ belongs to the full subcategory

$$
\mathcal{M} \otimes \mathrm{D}-\bmod _{\text {lisse }}\left(X^{I}\right) \subset \mathcal{M} \otimes \mathrm{D}-\bmod \left(X^{I}\right),
$$

\footnotetext{
${ }^{5}$ This assumption is not necessary if $\operatorname{Shv}(-)$ is sheaves in the classical topology.
} 
where $\mathrm{D}$-mod $\operatorname{misse}_{(-)} \subset \mathrm{D}$-mod(-) is the full subcategory of lisse D-modules (ind-completion of $\mathcal{O}$ coherent D-modules). For any $\mathcal{M}$, one can consider its maximal full subcategory $\mathcal{M}^{\text {lisse }} \subset \mathcal{M}$, on which the action is lisse.

Take $\mathcal{M}=\mathrm{D}-\bmod \left(\operatorname{Bun}_{G}\right)$. Theorem $\mathrm{B} .5 .2$ (due to $[\mathrm{NY}]$ ) says that we have an inclusion

$$
\mathrm{D}-\bmod _{\mathrm{Nilp}}\left(\operatorname{Bun}_{G}\right) \subset\left(\mathrm{D}-\bmod \left(\operatorname{Bun}_{G}\right)\right)^{\text {lisse }} .
$$

In Conjecture C.2.8 we propose that this inclusion is an equality.

Finally, we propose Conjecture C.3.7 that describes lisse actions in terms of set-theoretic support. Namely, let $\mathcal{M}$ be a category equipped with an action of $\operatorname{Rep}(\check{G})^{\otimes X}$ that factors via $\operatorname{QCoh}\left(\operatorname{LocSys}_{\breve{G}}(X)\right)$. Thus, given a compact object of $\mathcal{M}$, one can talk about its set-theoretic support, which is a Zariskiclosed subset in $\operatorname{LocSys}_{\tilde{G}}(X)$.

Conjecture C.3.7 says that a compact object of $\mathcal{M}$ belongs to $\mathcal{M}^{\text {lisse }}$ if and only if its set-theoretic support lies in the finite union of subsets induced from irreducible local systems for Levi subgroups of $G$.

0.3.10. In Sect. $\mathrm{D}$ we discuss the abstract notion of ULA, which has been used in the definition of ULA actions in Sect. C.

First, given a monoidal category $\mathcal{C}$ and a module category $\mathcal{M}$, we define what it means for an object $m \in \mathcal{M}$ to be ULA with respect to $\mathcal{C}$.

The case of interest for us is $\mathcal{C}=\mathrm{D}-\bmod (Y)$, with the monoidal structure given by the $\stackrel{!}{\otimes}$ operation.

We show that for $\mathcal{M}:=\mathrm{D}-\bmod (Z)$ for a scheme $Z$ over $Y$ we recover the usual notion of what it means for an object in $\mathrm{D}-\bmod (Z)$ to be ULA over $Y$.

We also establish the following criterion for ULA-ness: we recall that the datum of an D-mod $(Y)$ module category is equivalent to that of sheaf of categories over the de Rham prestack $Y_{\mathrm{dR}}$ of $Y$. In particular, we can consider the value of this sheaf of categories on $Y$ itself and obtain a $\mathrm{QCoh}(Y)$-module category $\mathcal{M}_{Y}$, which can be recovered from $\mathcal{M}$ as

$$
\mathcal{M}_{Y} \simeq \mathrm{QCoh}(Y) \underset{\mathrm{D}-\bmod (Y)}{\otimes} \mathcal{M} .
$$

In the case of $\mathcal{M}:=\mathrm{D}-\bmod (Z)$, we have:

$$
\mathcal{M}_{Y} \simeq \mathrm{QCoh}\left(Y \underset{Y_{\mathrm{dR}}}{\times} Z_{\mathrm{dR}}\right) ;
$$

when $Z \rightarrow Y$ is smooth, this is the (derived) category of modules over the sheaf of vertical differential operators.

The categories $\mathcal{M}$ and $\mathcal{M}_{Y}$ are related by an adjoint pair of functors

$$
\text { ind : } \mathcal{M}_{Y} \rightleftarrows \mathcal{M} \text { : oblv. }
$$

Our Theorem D.5.8 says that an object $m \in \mathcal{M}$ is ULA with respect to $\mathrm{D}-\bmod (Y)$ if and only if its image oblv $(m) \in \mathcal{M}_{Y}$ is compact.

\subsection{Notation and conventions.}

0.4.1. Higher categories. This paper will substantially use the language of $\infty$-categorie: 6 , as developed in Lu1.

We let Spc denote the $\infty$-category of spaces.

Given an $\infty$-category $\mathbf{C}$, and a pair of objects $\mathbf{c}_{1}, \mathbf{c}_{2} \in \mathbf{C}$, we let $\operatorname{Maps}_{\mathbf{C}}\left(\mathbf{c}_{1}, \mathbf{c}_{2}\right) \in \operatorname{Spc}$ be the mapping space between them. Given a space $Y$, by a $Y$-family of maps $\mathbf{c}_{1} \rightarrow \mathbf{c}_{2}$ we will mean a map $Y \rightarrow \operatorname{Maps}_{\mathbf{C}}\left(\mathbf{c}_{1}, \mathbf{c}_{2}\right)$ in Spc.

${ }^{6}$ We will often omit the adjective "infinity" and refer to $\infty$-categories simply as "categories" 
Recall that given an $\infty$-category $\mathbf{C}$ that contains filtered colimits, an object $\mathbf{c} \in \mathbf{C}$ is said to be compact if the Yoneda functor $\operatorname{Maps}_{\mathbf{C}}(\mathbf{c},-): \mathbf{C} \rightarrow$ Spc preserves filtered colimits. We let $\mathbf{C}^{c} \subset \mathbf{C}$ denote the full subcategory spanned by compact objects.

Given a functor $F: \mathbf{C}_{1} \rightarrow \mathbf{C}_{2}$ between $\infty$-categories, we will denote by $F^{R}$ (resp., $F^{L}$ ) its right (resp., left) adjoint, provided that it exists.

0.4.2. Higher algebra. Throughout this paper we will be concerned with higher algebra over a commutative ring of coefficients, denoted e. Although e is not necessarily a field, we will denote by Vect the stable $\infty$-category of chain complexes of e-modules, see, e.g., GaLu Example 2.1.4.8].

We will regard Vect as equipped with a symmetric monoidal structure (in the sense on $\infty$-categories), see, e.g., GaLu, Sect. 3.1.4]. Thus, we can talk about commutative/associative algebra objects in Vect, see, e.g., GaLu, Sect. 3.1.3].

Whenever we talk about algebraic geometry over e, we will mean derived algebraic geometry, built off derived affine schemes, the latter being by definition the category opposite to that of commutative algebras in Vect, connective with respect to the natural t-structure.

We will denote by DGCat the $\infty$-category of (presentable) cocomplete stable $\infty$-categories, equipped with a module structure over Vect with respect to the symmetric monoidal structure on the $\infty$-category of cocomplete stable $\infty$-categories given by the Lurie tensor product, see [Lu2, Sect. 4.8.1]. We will refer to objects of DGCat as "DG categories". We emphasize that as 1-morphisms in DGCat, only colimit-preserving functors are allowed.

For a given DG category $\mathcal{C}$, and a pair of objects $c_{1}, c_{2} \in \mathcal{C}$, we have a well-defined "inner Hom" object $\mathcal{H}_{\mathrm{e}}\left(c_{1}, c_{2}\right) \in$ Vect, characterized by the requirement that

$$
\operatorname{Maps}_{\text {Vect }}\left(V, \mathcal{H} \operatorname{Com}_{\mathcal{C}}\left(c_{1}, c_{2}\right)\right) \simeq \operatorname{Maps}_{\mathcal{C}}\left(V \otimes c_{1}, c_{2}\right), \quad V \in \operatorname{Vect} .
$$

The category DGCat itself carries a symmetric monoidal structure, given by Lurie tensor product over Vect. In particular, we can talk about the $\infty$-category of associative/commutative algebras in DGCat, which we denote by DGCat ${ }^{\text {Mon }}$ (resp., DGCat ${ }^{\text {SymMon }}$ ), and refer to as monoidal (resp., symmetric monoidal) DG categories.

Unless specified otherwise, all monoidal/symmetric monoidal DG categories will be assumed unital. Given a monoidal/symmetric monoidal DG category $\mathcal{A}$, we will denote by $\mathbf{1}_{\mathcal{A}}$ its unit object.

0.4.3. Rigidity. In multiple places in this paper we use the notion of rigidity for a monoidal DG category $\mathcal{A}$. We refer the reader to GR1 Sect. 9.1.1] for the general definition.

That said, in most of the cases of interest, the DG category in question will be compactly generated. In this case, according to [GR1, Lemma 9.1.5], the condition of being rigid is equivalent to the fact that its classes of compact objects and objects that are both left and right dualizable, coincide.

0.4.4. Prestacks. In Appendices $\mathrm{A}] \mathrm{B}$ we will deal with classical (i.e., non-derived) algebro-geometric objects over a ground field $k$ (which has nothing to do with our ring of coefficients e).

We will denote by $\operatorname{Sch}^{\text {aff }}$ the category of affine schemes (of finite type (!)) over $k$.

By a prestack (technically, prestack locally of finite type), we will mean an arbitrary functor

$$
\left(\mathrm{Sch}^{\mathrm{aff}}\right)^{\mathrm{op}} \rightarrow \mathrm{Spc} .
$$

0.5. Acknowledgements. We are grateful to A. Beilinson, V. Drinfeld, V. Lafforgue, J. Lurie and P. Scholze for valuable discussions, which informed our thinking about the subject.

The research of D.G. was supported by NSF grant DMS-1707662 and by Gelfand Chair at IHES. The research of Y.V. was supported by the ISF grant 822/17.

Part of the project was carried out while D.G, N.R and Y.V were at MSRI and were supported by NSF grant DMS-1440140.

The project have received funding from ERC under grant agreement No 669655. 


\section{Symmetric monoidal CAtegories integrated over a SPACE}

Let $\mathcal{A}$ be a symmetric monoidal category, and let $Y$ be an object of Spc. The goal of this section is to give an explicit description of the category

$$
\mathcal{A}^{\otimes Y}
$$

(sometimes also denoted $\int_{Y} \mathcal{A}$ ), which can be thought of as factorization homology of $\mathcal{A}$ along $Y$.

We will describe $\mathcal{A}^{\otimes Y}$ as a colimit as a (i) symmetric monoidal category, (ii) just monoidal category, (iii) plain DG category (each time the colimit will be taken within the corresponding ambient category, i.e., inside the category of symmetric monoidal categories, monoidal categories or DG categories, respectively).

In particular, we will give an explicit description of what it takes for $\mathcal{A}^{\otimes Y}$ to act (as a monoidal category) on a DG category $\mathcal{M}$, and what it takes to map out of $\mathcal{A}^{\otimes Y}$ as a plain DG category. Both descriptions will be formulated in terms of functors out of $\mathcal{A}^{\otimes I}$ parameterized by points of $Y^{I}$, for $I$ ranging over the category fSet of finite sets.

\subsection{The integral.}

1.1.1. Let $\mathbf{C}$ be a category with colimits. For an object $\mathbf{c} \in \mathbf{C}$ and $Y \in$ Spc define the object

$$
\int_{Y} \mathbf{c}:=\underset{Y}{\operatorname{colim}} \mathbf{c} \in \mathbf{C} \text {. }
$$

I.e., we take the colimit along the index category $Y$ of the constant functor $Y \rightarrow \mathbf{C}$ with value $\mathbf{c}$.

1.1.2. Tautologically, for $\mathbf{c}, \mathbf{c}^{\prime} \in \mathbf{C}$, we have

$$
\operatorname{Maps}_{\mathbf{C}}\left(\int_{Y} \mathbf{c}, \mathbf{c}^{\prime}\right) \simeq \operatorname{Maps}_{\mathrm{Spc}}\left(Y, \operatorname{Maps}_{\mathbf{C}}\left(\mathbf{c}, \mathbf{c}^{\prime}\right)\right) .
$$

This shows that the assignment

$$
\text { c, } Y \mapsto \int_{Y} \mathbf{c}
$$

is a functor

$$
\mathbf{C} \times \mathrm{Spc} \rightarrow \mathbf{C}
$$

Moreover, the functor (1.2) preserves colimits in each variable.

1.1.3. For a fixed $\mathbf{c}$, the functor

$$
Y \mapsto \int_{Y} \mathbf{c}, \quad \mathrm{Spc} \rightarrow \mathbf{C}
$$

can be characterized as the unique colimit-preserving functor whose value on $\{*\} \in \operatorname{Spc}$ is $\mathbf{c}$.

1.1.4. For example for $Y$ a discrete set $I$, we have

$$
\int_{I} \mathbf{c} \simeq \sqcup_{i \in I} \mathbf{c} .
$$


1.1.5. Let us recall the notion of left Kan extension. Let $i: \mathbf{D} \rightarrow \mathbf{D}^{\prime}$ be a functor; let $\mathbf{C}$ be a category with colimits, and let $F: \mathbf{D} \rightarrow \mathbf{C}$ be a functor. The left Kan extension of $F$ along $i$ is a functor

$$
\operatorname{LKE}_{i}(F): \mathbf{D}^{\prime} \rightarrow \mathbf{C}
$$

with the following universal property

$$
\operatorname{Maps}_{\text {Funct(D,C) }}(F, G \circ i) \simeq \operatorname{Maps}_{\text {Funct }\left(\mathbf{D}^{\prime}, \mathbf{C}\right)}\left(\operatorname{LKE}_{i}(F), G\right), \quad G \in \operatorname{Funct}\left(\mathbf{D}^{\prime}, \mathbf{C}\right) .
$$

One can show that the value of $\operatorname{LKE}_{i}(F)$ on an object $\mathbf{d}^{\prime} \in \mathbf{D}^{\prime}$ is given by

$$
\operatorname{colim}_{\mathbf{d} \in \mathbf{D} / \mathbf{d}^{\prime}} F(\mathbf{d}) \text {. }
$$

Here and elsewhere, the notation $\mathbf{D} / \mathbf{d}^{\prime}$ is the slice category, i.e., the category of

$$
\left\{\mathbf{d} \in \mathbf{D}, i(\mathbf{d}) \rightarrow \mathbf{d}^{\prime}\right\}
$$

1.1.6. Tautologically, we can rewrite the functor (1.3) as follows. Let $\{*\}$ be the point category, and let $*$ denote its single object. By a slight abuse of notation we will also denote by $\{*\}$ the corresponding object of Spc, i.e., the point space.

Then (1.3) is the left Kan extension along

$$
\{*\} \hookrightarrow \text { Spc, } \quad * \mapsto\{*\}
$$

of the functor

$$
\{*\} \rightarrow \mathbf{C}, \quad * \mapsto \mathbf{c} .
$$

1.1.7. Let fSet denote the category of finite sets, equipped with the embedding

$$
\text { fSet } \hookrightarrow \text { Spc . }
$$

By transitivity of the procedure of left Kan extension with respect to the composition

$$
\{*\} \hookrightarrow \text { fSet } \hookrightarrow \text { Spc, }
$$

we obtain that (1.3) identifies with the left Kan extension along (1.8) of its restriction to fSet, while the latter is given by (1.4).

\subsection{The tensor product $\mathcal{A}^{\otimes Y}$ as a colimit.}

1.2.1. We take $\mathbf{C}$ to be DGCat ${ }^{\text {SymMon }}$, so an object of $\mathbf{C}$ is a symmetric monoidal DG category $\mathcal{A}$. We wish to give an explicit description of the resulting category

$$
\mathcal{A}^{\otimes Y}:=\int_{Y} \mathcal{A}
$$

as a colimit, in three different contexts: (i) as a symmetric monoidal category, (ii) as a monoidal category, (iii) as a plain DG category.

1.2.2. For a category $\mathbf{D}$, let $\operatorname{Tw} \operatorname{Arr}(\mathbf{D})$ be the corresponding twisted arrows category. I.e., its objects are 1-morphisms in $\mathbf{D}$

$$
\mathbf{d}_{s} \rightarrow \mathbf{d}_{t},
$$

and morphisms

$$
\left(\mathbf{d}_{s}^{0} \rightarrow \mathbf{d}_{t}^{0}\right) \rightarrow\left(\mathbf{d}_{s}^{1} \rightarrow \mathbf{d}_{t}^{1}\right)
$$

are commutative diagrams

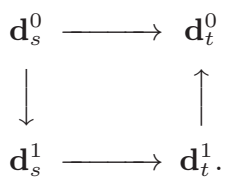

In practice we will take $\mathbf{D}$ to be the category fSet of finite sets. 
1.2.3. Let $\mathcal{A}$ be a symmetric monoidal category, and let $\mathbf{C}$ be one of the categories: DGCat ${ }^{\text {SymMon }}$, DGCat $^{\text {Mon }}$, DGCat. For a given $Y$, consider the functor

$$
\operatorname{TwArr}(\text { fSet }) \rightarrow \mathbf{C}, \quad(I \rightarrow J) \mapsto \int_{\operatorname{Maps}(J, Y)} \mathcal{A}^{\otimes I}
$$

We emphasize that in the above formula $\mathcal{A}^{\otimes I}$ is understood in the usual sense, i.e., the $I$-fold tensor product of $\mathcal{A}$ with itself, which is again a symmetric monoidal category, but viewed as an object in $\mathbf{C}$ using the tautological forgetful functor

$$
\text { DGCat }^{\text {SymMon }} \rightarrow \mathbf{C}
$$

while $\int_{\operatorname{Maps}(J, Y)}$ is taken within $\mathbf{C}$, i.e., the result of this operation looks different as a category, depending on which $\mathbf{C}$ we choose.

We claim:

Theorem 1.2.4. In each of the three cases in Sect.1.2.3, the image of $\mathcal{A}^{\otimes Y}$ under the forgetful functor

$$
\text { DGCat }^{\text {SymMon }} \rightarrow \mathbf{C}
$$

identifies canonically with the colimit in $\mathbf{C}$ of the functor 1.10 along TwArr(fSet).

Remark 1.2.5. The assertion of Theorem 1.2.4 with the same proof applies more generally, where instead of DGCat we consider a symmetric monoidal category $\mathbf{O}$ satisfying the conditions of Remark 1.3.7 below. So instead of DGCat ${ }^{\operatorname{SymMon}}$ we will have $\operatorname{ComAlg}(\mathbf{O})$ and $\mathbf{C}$ can be any of $\operatorname{ComAlg}(\mathbf{O})$, $\operatorname{Assoc} \operatorname{Alg}(\mathbf{O})$ or $\mathbf{O}$.

\subsection{Proof of Theorem 1.2.4}

1.3.1. Plan of the proof. We will show that both sides, viewed as functors $\mathrm{Spc} \rightarrow \mathbf{C}$, are left Kan extensions of their respective restrictions along (1.8). Then we will show that there restrictions are canonically isomorphic.

1.3.2. The first two steps will use the following general assertion. Recall that a category $A$ is said to be sifted if the diagonal functor $A \rightarrow A \times A$ is cofinal.

Lemma 1.3.3. If A has coproducts, then it is sifted.

Proof. We need to show that for any $a^{\prime}, a^{\prime \prime} \in A$, the category

$$
A_{\left(a^{\prime}, a^{\prime \prime}\right),}:=\left(a \in A, a^{\prime} \rightarrow a \leftarrow a^{\prime \prime}\right)
$$

is contractible7. Now, the fact that $A$ has coproducts means that $A_{\left(a^{\prime}, a^{\prime \prime}\right)}$, has an initial object.

Corollary 1.3.4. Let $\mathbf{D}$ have finite coproducts, and let $i: \mathbf{D} \rightarrow \mathbf{D}^{\prime}$ be a functor that preserves finite coproducts. Then for any $\mathbf{d}^{\prime} \in \mathbf{D}$, the slice category $\mathbf{D}_{/ \mathbf{d}^{\prime}}$ (see Equation (1.6)) is sifted.

Here is an application of Corollary 1.3.4 that we will use repeatedly:

Proposition 1.3.5. Let $i: \mathbf{D} \rightarrow \mathbf{D}^{\prime}$ be as in Corollary 1.3.4. Let $\mathbf{C}_{1}$ be a category that admits colimits, and let $\Phi: \mathbf{C}_{1} \rightarrow \mathbf{C}_{2}$ be a functor that preserves sifted colimits. Then for $F: \mathbf{D} \rightarrow \mathbf{C}_{1}$, the natural transformation

$$
\operatorname{LKE}_{i}(\Phi \circ F) \rightarrow \Phi \circ \operatorname{LKE}_{i}(F)
$$

obtained by the universal property of $\operatorname{LKE}_{i}(-)$, is an isomorphism.

Proof. Follows by Corollary 1.3.4 from the formula (1.5) for the values of $\operatorname{LKE}_{i}(-)$.

We now proceed to the proof of Theorem 1.2.4

\footnotetext{
${ }^{7}$ In our terminology "contractible" is what in Lu1 is called "weakly contractible".
} 
1.3.6. Step 1. Let us show that the functor

$$
\mathrm{Spc} \stackrel{Y \mapsto \mathcal{A}^{\otimes Y}}{\longrightarrow} \mathrm{DGCat}^{\mathrm{SymMon}} \rightarrow \mathbf{C}
$$

is the left Kan extension of its restriction along (1.8).

We apply Proposition 1.3.5 to the functor fSet $\rightarrow$ Spc, which obviously satisfies the assumption of Corollary 1.3.4.

Since the functor $Y \mapsto \mathcal{A}^{\otimes Y}$ is the left Kan extension of its restriction to fSet, it remains to show that the forgetful functor DGCat ${ }^{\text {SymMon }} \rightarrow \mathbf{C}$ preserves sifted colimits. This follows from the fact that both forgetful functors

$$
\text { DGCat }^{\text {SymMon }} \rightarrow \text { DGCat and DGCat }{ }^{\text {Mon }} \rightarrow \text { DGCat }
$$

preserve sifted colimits and are conservative.

Remark 1.3.7. The fact that the functors (1.11) preserve sifted colimits and are conservative is a consequence of the following:

Let $\mathbf{O}$ be a symmetric monoidal category which admits sifted colimits and such that the tensor product functor commutes with sifted colimits. Then for an operad $\mathcal{P}$, the forgetful functor

$$
\operatorname{oblv}_{\mathcal{P}}: \mathcal{P}-\operatorname{alg}(\mathbf{O}) \rightarrow \mathbf{O}
$$

preserves sifted colimits and is conservative, see [Lu2, Prop. 3.2.3.1 and Lemma 3.3.2.6].

1.3.8. Step 2. Let us now show that the functor $\mathrm{Spc} \rightarrow \mathbf{C}$ that sends $Y$ to the colimit (in $\mathbf{C}$ ) of the functor (1.10) along TwArr(fSet) is the left Kan extension of its restriction along (1.8).

It suffices to show that for each $I$ and $J$, the functor that sends $Y$ to $\int_{\operatorname{Maps}(J, Y)} \mathcal{A}^{\otimes I}$ is the left $\operatorname{Kan}$ extension of its restriction along (1.8). Since the functor (1.2) commutes with sifted colimits in the space variable, it suffices to show that the functor that sends $Y$ to $\operatorname{Maps}(J, Y)$ is the left $\operatorname{Kan}$ extension of its restriction along (1.8).

Since the functor $\operatorname{Maps}(J,-)$ preserves sifted colimits, this follows again from Proposition 1.3.5 applied to $\mathbf{C}_{1}=\mathbf{C}_{2}=$ Spc, using the fact that the identity functor on Spc is the left Kan extension of its restriction along (1.8).

1.3.9. Step 3. It remains to show that the restrictions of both sides in Theorem 1.2 .4 to fSet are canonically isomorphic.

However, this follows from the next version of the Yoneda lemma (which we prove below for the sake of completeness):

Proposition 1.3.10. Let $\mathbf{C}$ be a category with colimits and let $\Phi: \mathbf{D} \rightarrow \mathbf{C}$ be a functor. Then for $\mathbf{d} \in \mathbf{D}$ there is a canonical isomorphism

$$
\Phi(\mathbf{d}) \simeq \underset{\left(\mathbf{d}_{s} \rightarrow \mathbf{d}_{t}\right) \in \operatorname{TwArr}(\mathbf{D})}{\operatorname{colim}} \underset{\operatorname{Maps}\left(\mathbf{d}_{t}, \mathbf{d}\right)}{ } \Phi\left(\mathbf{d}_{s}\right) .
$$

$\square[$ Theorem 1.2 .4

1.3.11. For the proof of Proposition 1.3 .10 we note the following general feature of the twisted arrows category:

Let $F_{1}, F_{2}: \mathbf{D}^{\prime} \rightarrow \mathbf{E}$ be a pair of functors. Consider the functor

$$
\operatorname{Tw}\left(F_{1}, F_{2}\right): \operatorname{Tw} \operatorname{Arr}\left(\mathbf{D}^{\prime}\right)^{\mathrm{op}} \rightarrow \mathrm{Spc}
$$

that sends

$$
\left(\mathbf{d}_{s}^{\prime} \rightarrow \mathbf{d}_{t}^{\prime}\right) \mapsto \operatorname{Maps}_{\mathbf{E}}\left(F_{1}\left(\mathbf{d}_{s}^{\prime}\right), F_{2}\left(\mathbf{d}_{t}^{\prime}\right)\right) .
$$

We have the following standard fact (see e.g. GHN Prop. 5.1]): 
Lemma 1.3.12. There exists a canonical isomorphism

$$
\lim _{\operatorname{Tw} \operatorname{Arr}\left(\mathbf{D}^{\prime}\right)^{\mathrm{op}}} \operatorname{Tw}\left(F_{1}, F_{2}\right) \simeq \operatorname{Maps}_{\mathrm{Funct}\left(\mathbf{D}^{\prime}, \mathbf{E}\right)}\left(F_{1}, F_{2}\right) .
$$

1.3.13. To prove Proposition 1.3 .10 we apply Lemma 1.3 .12 to $\mathbf{D}^{\prime}:=\mathbf{D}^{\mathrm{op}}, \mathbf{E}=\mathrm{Spc}$ and

$$
F_{1}:=\operatorname{Maps}_{\mathbf{D}}(-, \mathbf{d}), F_{2}:=\operatorname{Maps}_{\mathbf{C}}(\Phi(-), \mathbf{c}), \quad \mathbf{c} \in \mathbf{C} .
$$

Note that we have a tautological identification

$$
\operatorname{Tw} \operatorname{Arr}(\mathbf{D}) \simeq \operatorname{Tw} \operatorname{Arr}\left(\mathbf{D}^{\mathrm{op}}\right)
$$

Using Lemma 1.3 .12 and (1.1), we obtain that

$$
\begin{aligned}
& \operatorname{Maps}_{\mathbf{C}}\left(\left(\underset{\left(\mathbf{d}_{s} \rightarrow \mathbf{d}_{t}\right) \in \operatorname{Tw} \operatorname{Arr}(\mathbf{D})}{\operatorname{colim}} \int_{\operatorname{Maps}\left(\mathbf{d}_{t}, \mathbf{d}\right)} \Phi\left(\mathbf{d}_{s}\right)\right), \mathbf{c}\right) \simeq \\
& \simeq \lim _{\left(\mathbf{d}_{s} \rightarrow \mathbf{d}_{t}\right) \in(\operatorname{TwArr}(\mathbf{D}))^{\mathrm{op}}} \operatorname{Maps}_{\mathbf{C}}\left(\int_{\operatorname{Maps}\left(\mathbf{d}_{t}, \mathbf{d}\right)} \Phi\left(\mathbf{d}_{s}\right), \mathbf{c}\right) \simeq \\
& \simeq \lim _{\left(\mathbf{d}_{s} \rightarrow \mathbf{d}_{t}\right) \in(\operatorname{TwArr}(\mathbf{D}))^{\mathrm{op}}} \operatorname{Maps}_{\mathrm{Spc}}\left(\operatorname{Maps}\left(\mathbf{d}_{t}, \mathbf{d}\right), \operatorname{Maps}\left(\Phi\left(\mathbf{d}_{s}\right), \mathbf{c}\right)\right) \simeq \\
& \simeq \operatorname{Maps}_{\text {Funct }\left(\mathbf{D}^{\mathrm{op}}, \mathrm{Spc}\right)}\left(\operatorname{Maps}_{\mathbf{D}}(-, \mathbf{d}), \operatorname{Maps}_{\mathbf{C}}(\Phi(-), \mathbf{c})\right),
\end{aligned}
$$

which by the usual Yoneda lemma identifies with

$$
\operatorname{Maps}_{\mathbf{C}}(\Phi(\mathbf{d}), \mathbf{c}),
$$

as desired.

1.4. The category of local systems. In this subsection we will describe the right adjoint to the functor

$$
\varrho \mapsto \int_{Y} \mathcal{e}, \quad \varrho \in \mathbf{C}
$$

for $\mathbf{C}$ being DGCat ${ }^{\mathrm{SymMon}}$, DGCat ${ }^{\mathrm{Mon}}$, DGCat.

1.4.1. For a category $\mathbf{C}$ with limits, an object $\mathbf{c} \in \mathbf{C}$ and $Y \in \mathrm{Spc}$, set

$$
\mathbf{c}^{Y}:=\lim _{Y} \mathbf{c},
$$

i.e., the limit of the functor $Y \rightarrow \mathbf{C}$ with constant value $\mathbf{c}$. For $Y$ being a discrete set $I$, we obtain

$$
\mathbf{c}^{I} \simeq \prod_{i \in I} \mathbf{c}
$$

Note that for $\mathbf{c}, \mathbf{c}^{\prime} \in \mathbf{C}$, we have

$$
\operatorname{Maps}_{\mathbf{C}}\left(\mathbf{c}^{\prime}, \mathbf{c}^{Y}\right) \simeq \operatorname{Maps}_{\mathrm{Spc}}\left(Y, \operatorname{Maps}_{\mathbf{C}}\left(\mathbf{c}^{\prime}, \mathbf{c}\right)\right) .
$$

The latter expression shows that the assignment

$$
\text { c, } Y \mapsto \mathbf{c}^{Y}
$$

is a functor

$$
\mathbf{C} \times \mathrm{Spc}^{\mathrm{op}} \rightarrow \mathbf{C},
$$

moreover, this functor preserves limits in each variable.

Furthermore, by (1.1), for a fixed $Y$, the functor

$$
\mathbf{c} \mapsto \mathbf{c}^{Y}, \quad \mathbf{C} \rightarrow \mathbf{C}
$$

is the right adjoint of the functor

$$
\mathbf{c}^{\prime} \mapsto \int_{Y} \mathbf{c}^{\prime}
$$


1.4.2. Take $\mathbf{C}=$ DGCat $^{\text {SymMon }}$ and set

$$
\operatorname{LS}(Y):=\operatorname{Vect}^{Y} \in \mathrm{DGCat}^{\text {SymMon }} .
$$

Since the forgetful functors

$$
\mathrm{DGCat}^{\text {SymMon }} \rightarrow \mathrm{DGCat}^{\text {Mon }} \rightarrow \text { DGCat }
$$

commute with limits (this is valid in the general context of algebras over an operad in Sect. 1.3.6 see Lu2, Prop. 3.2.2.1]), the image of $\operatorname{LS}(Y)$ under these forgetful functors identifies with $\operatorname{Vect}^{Y}$, thought of taking values in DGCat ${ }^{\text {Mon }}$ (resp., DGCat).

Remark 1.4.3. Let $y$ be a topological space 8 weakly equivalent to the geometric realization of $Y$. One can show that $\operatorname{LS}(Y)$ is equivalent to the full subcategory $\operatorname{Shv}_{\text {lisse }}(y)$ of the (unbounded) derived category $\operatorname{Shv}(y)$ of sheaves of e-vector spaces on $y$ consisting of objects with locally constant cohomology sheaves.

1.4.4. We will now describe the category $\operatorname{LS}(Y)$ as a plain DG category more explicitly:

Proposition 1.4.5.

(a) For a map $f: Y_{1} \rightarrow Y_{2}$, the restriction map $f^{\dagger}: \operatorname{LS}\left(Y_{2}\right) \rightarrow \operatorname{LS}\left(Y_{1}\right)$ admits a left adjoint.

(a') The functor

$$
\mathrm{Spc} \rightarrow \text { DGCat }, \quad Y \mapsto \operatorname{LS}(Y), \quad\left(Y_{1} \stackrel{f}{\rightarrow} Y_{2}\right) \rightsquigarrow\left(\operatorname{LS}\left(Y_{1}\right) \stackrel{\left(f^{\dagger}\right)^{L}}{\longrightarrow} \operatorname{LS}\left(Y_{2}\right)\right)
$$

is canonically isomorphic to the functor

$$
Y \mapsto \int_{Y} \text { Vect }
$$

(b) The category $\operatorname{LS}(Y)$ is dualizable.

(b') The functor (1.12) is canonically isomorphic to the functor

$$
\mathrm{Spc} \rightarrow \text { DGCat }, \quad Y \mapsto \operatorname{LS}(Y)^{\vee}, \quad\left(Y_{1} \stackrel{f}{\rightarrow} Y_{2}\right) \rightsquigarrow\left(\operatorname{LS}\left(Y_{1}\right)^{\vee} \stackrel{\left(f^{\dagger}\right)^{\vee}}{\longrightarrow} \operatorname{LS}\left(Y_{2}\right)^{\vee}\right)
$$

The proof of this proposition is given below.

Corollary 1.4.6. The category $\int_{Y}$ Vect is dualizable. The functor

$$
\mathrm{Spc}^{\mathrm{op}} \rightarrow \text { DGCat }, \quad Y \mapsto\left(\int_{Y} \text { Vect }\right)^{\vee}, \quad\left(Y_{1} \rightarrow Y_{2}\right) \rightsquigarrow\left(\left(\int_{Y_{2}} \text { Vect }\right)^{\vee} \rightarrow\left(\int_{Y_{1}} \text { Vect }\right)^{\vee}\right)
$$

is canonically isomorphic to the functor $Y \mapsto \operatorname{LS}(Y)$.

Proof. Combine points (a') and (b') of Proposition 1.4.5

Note that in particular, Proposition 1.4.5 and Corollary 1.4.6 imply that for an individual $Y$ we have the canonical equivalences

$$
\operatorname{LS}(Y) \simeq \int_{Y} \text { Vect, } \operatorname{LS}(Y)^{\vee} \simeq \operatorname{LS}(Y), \quad\left(\int_{Y} \operatorname{Vect}\right)^{\vee} \simeq \int_{Y} \text { Vect } .
$$

${ }^{8}$ We assume that $y$ is sufficiently nice; i.e. a paracompact topological space homotopy equivalent to a CW complex and of finite homotopical dimension (see Lu1 Sect. 7.1]) 
1.4.7. The proof of Proposition 1.4.5 is based on the next general lemma (see GR1, Chapter 1, Propositions 2.5.7 and 6.3.4, and Lemma 2.6.4]):

Lemma 1.4.8. Let

$$
a \mapsto \mathcal{C}_{a}, \quad A \rightarrow \text { DGCat }
$$

be a diagram of $D G$ categories, such that for every arrow $a_{1} \rightarrow a_{2}$, the corresponding functor

$$
F_{a_{1}, a_{2}}: \mathcal{C}_{a_{1}} \rightarrow \mathcal{C}_{a_{2}}
$$

admits a continuous right adjoint. Set

$$
\mathcal{C}:=\underset{a \in A}{\operatorname{colim}} \mathcal{C}_{a}
$$

Then:

(a) The tautological functors $\operatorname{ins}_{a}: \mathcal{C}_{a} \rightarrow \mathcal{C}$ admit continuous right adjoints.

(b) Consider the functor

$$
A^{\mathrm{op}} \rightarrow \text { DGCat, } \quad a \mapsto \mathcal{C}_{a}, \quad\left(a_{1} \rightarrow a_{2}\right) \rightsquigarrow\left(\mathcal{C}_{a_{2}} \stackrel{F_{a_{1}, a_{2}}^{R}}{\longrightarrow} \mathcal{C}_{a_{1}}\right)
$$

and set

$$
\widetilde{\mathrm{C}}:=\lim _{a \in A^{\mathrm{op}}} \mathcal{C}_{a}
$$

Then the functor $\mathrm{e} \rightarrow \widetilde{\mathfrak{C}}$, given by the system of functors

$$
\operatorname{ins}_{a}^{R}: \mathcal{C} \rightarrow \mathcal{C}_{a}
$$

is an equivalence.

(c) Let $\phi: A^{\prime} \rightarrow A$ be a functor of index categories. Composing with (1.14) and (1.15), respectively, we obtain the functors

$$
A^{\prime} \rightarrow \text { DGCat and } A^{\prime \text { op }} \rightarrow \text { DGCat }
$$

Set

$$
\mathcal{C}^{\prime}:=\operatorname{colim}_{a^{\prime} \in A^{\prime}} \mathcal{C}_{\phi\left(a^{\prime}\right)} \text { and } \widetilde{\mathfrak{C}}^{\prime}:=\lim _{a^{\prime} \in A^{\prime o p}} \mathcal{C}_{\phi\left(a^{\prime}\right)}
$$

Then under the identifications

$$
\mathrm{e} \simeq \widetilde{\mathrm{C}} \text { and } \mathrm{e}^{\prime} \simeq \widetilde{\mathrm{e}}^{\prime}
$$

the tautological functor $\mathrm{C}^{\prime} \rightarrow \mathcal{C}$ identifies with the left adjoint of the restriction functor $\widetilde{\mathcal{C}}^{\prime} \rightarrow \widetilde{\mathcal{C}}$.

(d) Assume that all $\mathrm{C}_{a}$ are dualizable. Consider the functor

$$
A^{\text {op }} \rightarrow \text { DGCat, } \quad a \mapsto \mathcal{C}_{a}^{\vee}, \quad\left(a_{1} \rightarrow a_{2}\right) \rightsquigarrow\left(\mathcal{C}_{a_{2}}^{\vee} \stackrel{F_{a_{1}, a_{2}}^{\vee}}{\longrightarrow} \mathcal{C}_{a_{1}}^{\vee}\right)
$$

Then $\mathrm{C}$ is dualizable, and the functor

$$
\mathrm{C}^{\vee} \rightarrow \lim _{a \in A^{\text {op }}} \mathcal{C}_{a}^{\vee}
$$

given by the system of functors

$$
\operatorname{ins}_{a}^{\vee}: \mathcal{C}^{\vee} \rightarrow \mathcal{C}_{a}^{\vee}
$$

is an equivalence.

Proof of Proposition 1.4.5. Recall that

$$
\int_{Y} \text { Vect } \simeq \text { colim Vect and } \operatorname{LS}(Y) \simeq \lim _{Y} \text { Vect } .
$$

The identification

$$
\int_{Y} \operatorname{Vect} \simeq \operatorname{LS}(Y)
$$

follows from Lemma 1.4.8(b) applied to the index category $Y$ and the constant family with value Vect.

Point (a') of Proposition 1.4.5 follows from Lemma 1.4.8 (c), implying also point (a).

Point (b') of Proposition 1.4.5 follows from Lemma 1.4.8 (d), implying also point (b). 
1.4.9. Let $\mathbf{C}$ be one of the three categories from Theorem 1.2 .4 and let $\mathcal{C}$ be an object of $\mathbf{C}$. I.e., $\mathcal{C}$ is either a symmetric monoidal category, or a monoidal category or a plain DG category.

Note that for a symmetric monoidal category $\mathcal{A}$, the tensor product $\mathcal{C} \otimes \mathcal{A}$ is again naturally an object of $\mathbf{C}$ (this is true for DGCat replaced by an arbitrary symmetric monoidal category $\mathbf{O}$ and any operad $\mathcal{P}$ as in Sect. 1.3.6 this follows, e.g., from [Lu2, Prop. 3.2.4.3]).

We claim that we have a natural map

$$
\mathcal{e} \otimes \operatorname{LS}(Y) \rightarrow \mathrm{e}^{Y}
$$

Indeed, by the definition of $\mathrm{e}^{Y}$, the datum of such a map is equivalent to that of a family of maps $\mathcal{C} \otimes \operatorname{LS}(Y) \rightarrow \mathcal{C}$, parameterized by points of $Y$. For $y \in Y$ we take the corresponding map to be

$$
\mathcal{C} \otimes \operatorname{LS}(Y) \stackrel{\operatorname{Id}_{\mathcal{e}} \otimes \operatorname{ev}_{y}}{\longrightarrow} \mathcal{e} \otimes \operatorname{Vect} \simeq \mathcal{e}
$$

where

$$
\operatorname{ev}_{y}: \operatorname{LS}(Y) \rightarrow \operatorname{LS}(\{*\}) \simeq \operatorname{Vect}
$$

is the functor of pullback corresponding to $\{*\} \stackrel{y}{\rightarrow} Y$.

We claim:

Proposition 1.4.10. The map (1.17) is an isomorphism.

The proof of Proposition 1.4.10 is given below.

Using Sect. 1.4.1 from Proposition 1.4.10 we obtain:

Corollary 1.4.11. For a given $Y$, the right adjoint of the functor

$$
\mathrm{e}^{\prime} \mapsto \int_{Y} \mathrm{e}^{\prime}
$$

is given by

$$
\mathcal{C} \mapsto \mathcal{C} \otimes \operatorname{LS}(Y)
$$

Proof of Proposition 1.4.10. Since the forgetful functor $\mathbf{C} \rightarrow$ DGCat commutes with the operation

$$
-\otimes \operatorname{LS}(Y)
$$

it suffices to prove the assertion for $\mathbf{C}=$ DGCat.

The map in question is an isomorphism for $Y=\{*\}$. Since $Y \simeq \operatorname{colim}_{Y}\{*\}$, it suffices to show that (for a fixed $\mathcal{C}$ ), the functor

$$
\mathcal{C} \mapsto \mathcal{C} \otimes \operatorname{LS}(Y)
$$

takes colimits in $Y$ to limits in DGCat. However, this follows from Corollary 1.4.6 as the functor in question can be rewritten as

$$
\text { Funct }\left(\int_{Y} \text { Vect, } \mathrm{C}\right) \text {. }
$$

1.5. Digression: the stack of G-local systems. In this subsection we will assume that the ring e of coefficients is a field of characteristic 0 .

1.5.1. Let $\mathrm{G}$ be an affine group-scheme over e. We let $\operatorname{Rep}(\mathrm{G})$ be the category of G-representations, defined as

$$
\operatorname{Rep}(\mathrm{G}):=\mathrm{QCoh}(\mathrm{pt} / \mathrm{G}) .
$$

The assumption on e implies that the category $\operatorname{Rep}(G)$ is compactly generated; its compact objects are those that are mapped to compact objects of Vecte under the forgetful functor

$$
\operatorname{oblv}_{\mathrm{G}}: \operatorname{Rep}(\mathrm{G}) \rightarrow \operatorname{Vect}=\mathrm{QCoh}(\mathrm{pt}) .
$$


1.5.2. For $Y \in \mathrm{Spc}$, let $\operatorname{LocSys}_{\mathrm{G}}(Y)$ denote the (derived) Artin stack that classifies G-local systems on $Y$. By definition, for a derived affine scheme $S$,

$$
\operatorname{Maps}\left(S, \operatorname{LocSys}_{\mathrm{G}}(Y)\right)
$$

is the space of right t-exact symmetric monoidal functors

$$
\operatorname{Rep}(\mathrm{G}) \rightarrow \mathrm{QCoh}(S) \otimes \operatorname{LS}(Y),
$$

(where the RHS is equipped with the tensor product t-structure, see [Lu3, Sect. C.4]).

Consider the (symmetric monoidal) category $\mathrm{QCoh}\left(\operatorname{LocSys}_{\mathrm{G}}(Y)\right)$. We will now construct a (symmetric monoidal) functor

$$
\operatorname{Rep}(\mathrm{G})^{\otimes Y} \rightarrow \mathrm{QCoh}\left(\operatorname{LocSys}_{\mathrm{G}}(Y)\right) .
$$

1.5.3. First, we note that for $Y=\{*\}$, we have

$$
\operatorname{LocSys}_{\mathrm{G}}(\{*\}) \simeq \mathrm{pt} / \mathrm{G} .
$$

$\operatorname{Indeed,~} \operatorname{Maps}(S, \mathrm{pt} / \mathrm{G})$ is the groupoid of G-torsors on $S$, and those can be described as right t-exact symmetric monoidal functors

see [AG, Sect. 10.2].

$$
\operatorname{Rep}(\mathrm{G}) \rightarrow \mathrm{QCoh}(S)
$$

Hence,

$$
\mathrm{QCoh}\left(\operatorname{LocSys}_{\mathrm{G}}(\{*\})\right) \simeq \mathrm{QCoh}(\mathrm{pt} / \mathrm{G})=: \operatorname{Rep}(\mathrm{G}) .
$$

For any $Y$, we have a canonical map of spaces

$$
\begin{aligned}
& Y \simeq \operatorname{Maps}_{\mathrm{Spc}}(\{*\}, Y) \rightarrow \operatorname{Maps}_{\mathrm{PreStk}}\left(\operatorname{LocSys}_{\mathrm{G}}(Y), \operatorname{LocSys}_{\mathrm{G}}(\{*\})\right) \rightarrow
\end{aligned}
$$

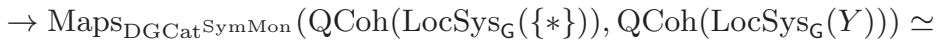

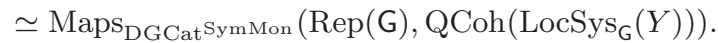

Hence, by (1.1), we obtain the desired map

$$
\operatorname{Rep}(\mathrm{G})^{\otimes Y}:=\int_{Y} \operatorname{Rep}(\mathrm{G}) \rightarrow \operatorname{QCoh}\left(\operatorname{LocSys}_{\mathrm{G}}(Y)\right) .
$$

1.5.4. We claim:

Theorem 1.5.5. Assume that $Y$ has finitely many connected components. Then the functor (1.19) is an equivalence.

Remark 1.5.6. The assertion of Theorem 1.5.5 would fail for $Y$ which is the infinite disjoint union of copies of $\{*\}$, and $\mathrm{G}=\mathbb{G}_{a}$.

1.5.7. We will actually prove a generalization of Theorem 1.5 .5 Let $z$ be a prestack satisfying the following conditions:

- The diagonal map $z \rightarrow z \times z$ is affine;

- The category $\mathrm{QCoh}(z)$ is dualizable;

Let us call such prestacks quasi-passable, cf. GR1, Chapter 3, Sect. 3.5.1].

For $Y \in \operatorname{Spc}$ consider the prestack $\operatorname{Maps}(Y, Z)$ defined as

$$
\operatorname{Maps}_{\text {PreStk }}(S, \operatorname{Maps}(Y, Z))=\operatorname{Maps}_{\mathrm{Spc}}\left(Y, \operatorname{Maps}_{\text {PreStk }}(S, Z)\right), \quad S \in \operatorname{Sch}^{\text {aff }} .
$$

As in Sect. 1.5.3 we have a canonically defined symmetric monoidal functor

$$
\mathrm{QCoh}(Z)^{\otimes Y} \rightarrow \mathrm{QCoh}(\operatorname{Maps}(Y, Z))
$$

We will prove:

Theorem 1.5.8. Assume that $Y$ has finitely many connected components and that $Z$ is quasi-passable. Then the prestack $\operatorname{Maps}(Y, Z)$ is quasi-passable and the functor (1.20) is an equivalence. 
Theorem 1.5.5 is a particular case of Theorem 1.5 .8 for $z=p t / G$; note that in this case

$$
\operatorname{Maps}(Y, \mathrm{pt} / \mathrm{G}) \simeq \operatorname{LocSys}_{\mathrm{G}}(Y) \text {. }
$$

\subsection{Proof of Theorem 1.5 .8}

1.6.1. We will first establish a few basic facts about quasi-passable prestacks.

Lemma 1.6.2. Suppose that $f: z_{1} \rightarrow z_{2}$ is an affine morphism, where $z_{2}$ is quasi-passable. Then $z_{1}$ is quasi-passable.

Proof. By the standard argument, $z_{1}$ has affine diagonal; namely, by assumption, the maps $z_{1} \rightarrow$ $z_{2} \rightarrow z_{2} \times z_{2}$ and $z_{1} \times z_{1} \rightarrow z_{2} \times z_{2}$ are affine and by the 2 -out-of- 3 propery of affine morphisms, we obtain that $z_{1} \rightarrow z_{1} \times z_{1}$ is affine, as desired.

It remains to show that $\mathrm{QCoh}\left(z_{1}\right)$ is dualizable. By GR1 Chapter 3, Proposition 3.3.3], we have

$$
\mathrm{QCoh}\left(z_{1}\right) \simeq f_{*}\left(\mathcal{O}_{Z_{1}}\right)-\bmod \left(\mathrm{QCoh}\left(z_{2}\right)\right) \text {. }
$$

The lemma follows from the following general fact (applied to the monad given by tensoring with $\left.f_{*}\left(\mathcal{O}_{Z_{1}}\right)\right)$.

Lemma 1.6.3. Let $\mathbf{C}$ be a dualizable $D G$ category and let $M: \mathbf{C} \rightarrow \mathbf{C}$ be a monad such that the underlying endo-functor of $\mathbf{C}$ preserves colimits. Then the category $M-\bmod (\mathbf{C})$ is dualizable.

Proof. It is easy to see that $M^{\vee}-\bmod \left(\mathbf{C}^{\vee}\right)$ provides a dual for $M-\bmod (\mathbf{C})$.

1.6.4. As a consequence, we have following persistence property of quasi-passable prestacks:

Corollary 1.6.5. Suppose that we have a pullback diagram

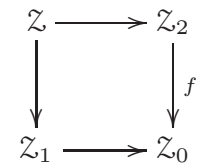

such that $z_{0}, z_{1}$ and $z_{2}$ are quasi-passable and the map $f: z_{2} \rightarrow z_{0}$ is affine. Then $z$ is quasi-passable and the functor

is an equivalence.

$$
\mathrm{QCoh}\left(z_{1}\right) \underset{\mathrm{QCoh}\left(z_{0}\right)}{\otimes} \mathrm{QCoh}\left(z_{2}\right) \rightarrow \mathrm{QCoh}(z)
$$

Proof. By base change, the map $z \rightarrow z_{1}$ is affine and therefore $z$ is quasi-passable by Lemma 1.6 .2 Moreover, by base change and [GR1, Chapter 3, Proposition 3.3.3], we have

$$
\begin{gathered}
\operatorname{QCoh}(z) \simeq f_{*}\left(\mathcal{O}_{z_{2}}\right)-\bmod \left(\operatorname{QCoh}\left(z_{1}\right)\right) \simeq f_{*}\left(\mathcal{O}_{z_{2}}\right)-\bmod \left(\operatorname{QCoh}\left(z_{0}\right)\right) \underset{\mathrm{QCoh}\left(z_{0}\right)}{\otimes} \operatorname{QCoh}\left(z_{1}\right) \simeq \\
\simeq \operatorname{QCoh}\left(z_{1}\right) \underset{\mathrm{QCoh}\left(z_{0}\right)}{\otimes} \operatorname{QCoh}\left(z_{2}\right),
\end{gathered}
$$

as desired.

1.6.6. We are now ready to prove the Theorem 1.5.8 We first reduce to the case that $Y$ is connected. Indeed, by [GR1, Chapter 3, Theorem 3.1.7] if $X_{1}$ and $X_{2}$ are quasi-passable prestacks, so is $X_{1} \times X_{2}$ and the functor

$$
\mathrm{QCoh}\left(x_{1}\right) \otimes \mathrm{QCoh}\left(x_{2}\right) \rightarrow \mathrm{QCoh}\left(x_{1} \times x_{2}\right)
$$

is an equivalence.

In particular, if $Y=Y_{1} \sqcup Y_{2}$, we have

$$
\operatorname{Maps}\left(Y_{1} \sqcup Y_{2}, Z\right) \simeq \operatorname{Maps}\left(Y_{1}, Z\right) \times \operatorname{Maps}\left(Y_{2}, Z\right)
$$

Therefore, if Theorem 1.5.8 is true for $Y_{1}$ and $Y_{2}$, it is true for $Y$ (using the fact that the coproduct in DGCat ${ }^{\text {SymMon }}$ is given by tensor product). 
1.6.7. We can thus assume without loss of generality that $Y$ is connected. In this case, we will prove a slightly more precise version of the theorem (which implies Theorem 1.5.8 by Lemma 1.6.2):

Theorem 1.6.8. Let $(Y, y)$ be a pointed connected space and $z$ a quasi-passable prestack. Then the evaluation map

$$
e v_{y}: \operatorname{Maps}(Y, Z) \rightarrow Z
$$

is affine and the functor (1.20) is an equivalence.

1.6.9. By Proposition 2.1.5 below, we can write $(Y, y)$ as a sifted colimit

$$
(Y, y)=\operatorname{colim}_{\alpha \in A}\left(Y_{\alpha}, y\right)
$$

in $\mathrm{Spc}_{\mathrm{Ptd}}$ (and therefore $\mathrm{Spc}$ ) of objects of the form

$$
\left(Y_{\alpha}, y\right)=\left(\{*\} \simeq\{*\} \underset{\{*\}}{\sqcup}\{*\} \rightarrow\{*\} \underset{I \sqcup\{*\}}{\sqcup}\{*\} \simeq \underset{I}{\vee} S^{1}\right), \quad I \in \text { fSet } .
$$

This gives an isomorphism of prestacks

$$
\operatorname{Maps}(Y, Z) \simeq \lim _{\alpha \in A} \operatorname{Maps}\left(Y_{\alpha}, Z\right) .
$$

Theorem 1.6.8 now follows from the following two assertions:

Lemma 1.6.10. Theorem 1.6 .8 is true for $Y=\underset{I}{\vee} S^{1}, I \in$ fSet.

Lemma 1.6.11. Let $a \mapsto \mathcal{W}_{a}$ be a sifted diagram of prestacks, affine over some base prestack $Z$; set

$$
\mathcal{W}:=\lim _{a} \mathcal{W}_{a} .
$$

Then the map $\mathcal{W} \rightarrow Z$ is affine and the induced functor

$$
\underset{a}{\operatorname{colim}} \mathrm{QCoh}\left(\mathcal{W}_{a}\right) \rightarrow \mathrm{QCoh}(\mathcal{W})
$$

is an equivalence.

1.6.12. Proof of Lemma 1.6.10. Since $\underset{I}{\vee} S^{1} \simeq\{*\} \underset{I \sqcup\{*\}}{\sqcup}\{*\}$, we have a pullback diagram of prestacks

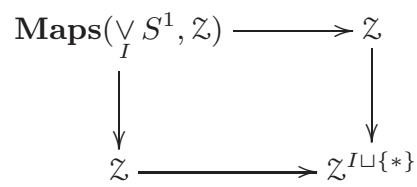

Since $z$ is quasi-passable, so is $z^{I \sqcup\{*\}}$ and the diagonal map $z \rightarrow z^{I \sqcup\{*\}}$ is affine. The result then follows by Corollary 1.6.5 (using the fact the pushouts in DGCat ${ }^{\text {SymMon }}$ are given by tensor products).

1.6.13. Proof of Lemma 1.6.11. The assertion that $\mathcal{W} \rightarrow Z$ is affine follows from the fact a limit of affine schemes is an affine scheme and limits commute with pullbacks.

Now, let $\mathcal{A}_{a}$ (resp., $\left.\mathcal{A}\right)$ be the object of $\operatorname{ComAlg}(\mathrm{QCoh}(Z))$ equal to the image of the structure sheaf on $\mathcal{W}_{\alpha}$ (resp., $\left.\mathcal{W}\right)$. Then (e.g., by GR1 Chapter 3, Prop. 3.3.3]), direct image induces an equivalence

$$
\mathrm{QCoh}\left(\mathcal{W}_{a}\right) \simeq \mathcal{A}_{a}-\bmod (\mathrm{QCoh}(z)) \text { and } \mathrm{QCoh}(\mathcal{W}) \simeq \mathcal{A}-\bmod (\mathrm{QCoh}(z))
$$

Now the assertion of the lemma follows from Lemma 2.5.2 below, combined with the fact that the forgetful functor

commutes with sifted colimits.

$$
\text { ComAlg } \rightarrow \text { AssocAlg }
$$

1.7. Functors out of $\mathcal{A}^{\otimes Y}$ as diagrams parameterized by finite sets. In this subsection we will derive some corollaries of Theorem 1.2.4 
1.7.1. Let $\mathbf{C}$ be one of the three categories from Theorem 1.2.4 and let $\mathcal{C}$ be an object of $\mathbf{C}$. I.e., $\mathcal{C}$ can be a symmetric monoidal DG category, or a monoidal DG category, or a plain DG category.

Consider the following two functors

$$
\begin{aligned}
& \text { fSet } \rightarrow \mathbf{C}, \\
& I \mapsto \mathcal{A}^{\otimes I} ;
\end{aligned}
$$

and

$$
I \mapsto \mathcal{C} \otimes \operatorname{LS}\left(Y^{I}\right)
$$

We claim:

Proposition 1.7.2. The space of maps $\mathcal{A}^{\otimes Y} \rightarrow \mathcal{C}$ within $\mathbf{C}$ (i.e., functors $\mathcal{A}^{\otimes Y} \rightarrow \mathcal{C}$ that preserve the corresponding structure) is canonically isomorphic to the space of natural transformations from (1.22) to (1.23).

Proof. Recall (see Lemma 1.3.12) that given a pair of functors

$$
\Phi_{1}, \Phi_{2}: \mathbf{D} \rightarrow \mathbf{C},
$$

the space of natural transformations $\Phi_{1} \rightarrow \Phi_{2}$ identifies with the limit

$$
\lim _{\left(\mathbf{d}_{s} \rightarrow \mathbf{d}_{t}\right) \in \operatorname{Tw} \operatorname{Arr}(\mathbf{D})^{\mathrm{op}}} \operatorname{Maps}_{\mathbf{C}}\left(\Phi_{1}\left(\mathbf{d}_{s}\right), \Phi_{2}\left(\mathbf{d}_{t}\right)\right) .
$$

Hence, we obtain that the space of natural transformations from (1.22) to (1.23) identifies canonically with

$$
\lim _{(I \rightarrow J) \in \operatorname{TwArr}(\mathrm{fSet}) \text { op }} \operatorname{Maps}_{\mathbf{C}}\left(\mathcal{A}^{\otimes I}, \mathcal{C} \otimes \operatorname{LS}\left(Y^{J}\right)\right) .
$$

Applying Corollary 1.4.11 we rewrite this as

$$
\lim _{(I \rightarrow J) \in \operatorname{TwArr}(\text { fSet })^{\text {op }}} \operatorname{Maps}_{\mathbf{C}}\left(\int_{Y^{J}} \mathcal{A}^{\otimes I}, \mathcal{C}\right) .
$$

Now the assertion follows from Theorem 1.2.4 which says that

$$
\underset{(I \rightarrow J) \in \operatorname{TwArr}(\mathrm{fSet})}{\operatorname{colim}} \int_{Y^{J}} \mathcal{A}^{\otimes I} \simeq \mathcal{A}^{\otimes Y} .
$$

1.7.3. As a first application of Proposition 1.7.2 we will describe what it takes to have an action of $\mathcal{A}^{\otimes Y}$ on some DG category $\mathcal{M}$.

We take $\mathbf{C}=$ DGCat $^{\text {Mon }}$. Set $\mathcal{C}:=\operatorname{End}(\mathcal{M})$; this is a monoidal category. An action of $\mathcal{A}^{\otimes Y}$ on $\mathcal{M}$ is the same as a map of monoidal categories

$$
\mathcal{A}^{\otimes Y} \rightarrow \operatorname{End}(\mathcal{M})
$$

According to Proposition 1.7.2, the latter amounts to a system of monoidal functors

$$
\mathcal{A}^{\otimes I} \rightarrow \operatorname{End}(\mathcal{M}) \otimes \operatorname{LS}\left(Y^{I}\right), \quad I \in \mathrm{fSet},
$$

compatible in the sense that they make the following diagrams commute

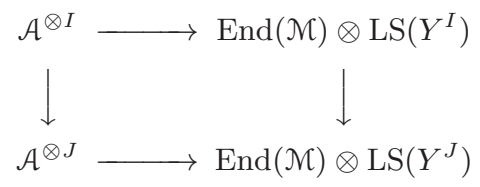

for every $I \rightarrow J$, along with a system of higher compatibilities. 
Remark 1.7.4. Let us note the difference between the notion of action of $\mathcal{A}^{\otimes Y}$ on $\mathcal{M}$, and that of action of $\int_{Y} \mathcal{A}$ on $\mathcal{M}$, where $\int_{Y}-$ is understood within DGCat $^{\mathrm{Mon}}$ :

The former contains the data of the homomorphisms (1.24) for all $I$ with the compatibilities expressed by diagrams (1.25). The latter contains just the data of homomorphism

$$
\mathcal{A} \rightarrow \operatorname{End}(\mathcal{M}) \otimes \operatorname{LS}(Y)
$$

i.e., the case $I=\{*\}$.

1.7.5. Example of $Y=S^{1}$. Let us elaborate further on Remark 1.7.4 in the case $Y=S^{1}$. Note that by identifying

$$
S^{1}=\{*\}_{\{*\} \sqcup\{*\}}^{\sqcup}\{*\},
$$

we obtain that $\operatorname{LS}\left(S^{1}\right)$ identifies with the category of vector spaces equipped with an automorphism.

The monoidal category $\operatorname{End}(\mathcal{M}) \otimes \operatorname{LS}\left(S^{1}\right)$ is that of endofunctors of $\mathcal{M}$ equipped with an automorphism. Hence, an action of $\int_{S^{1}} \mathcal{A}$ (with $\int$ understood as taking place inside DGCat ${ }^{\text {Mon }}$ ) on $\mathcal{M}$ is an assignment

$$
a \in \mathcal{A} \mapsto\left(H_{a}, \operatorname{mon}_{a}\right),
$$

where $H_{a}$ is an endofunctor of $\mathcal{M}$ equipped with an endomorphism $\operatorname{mon}_{a}$. These data must be compatible in the sense that for $m \in \mathcal{M}$ the diagram

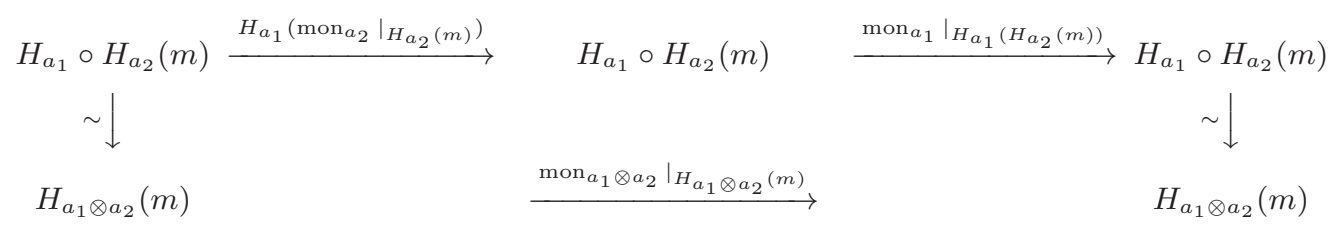

should commute, along with higher compatibilities. In the case when $\mathcal{M}$ and $\mathcal{A}$ are derived categories of abelian categories and the action is t-exact, the commutativity of the diagrams (1.26) for objects in the heart is sufficient to ensure the higher compatibilities.

By contrast, an action of $\mathcal{A}^{\otimes S^{1}}$ on $\mathcal{M}$ requires additional compatibilities. The first of these is that under the identification

$$
H_{a_{1}} \circ H_{a_{2}}(m) \simeq H_{a_{1} \otimes a_{2}}(m) \simeq H_{a_{2} \otimes a_{1}}(m) \simeq H_{a_{2}} \circ H_{a_{1}}(m)
$$

the automorphism

$$
\left.\operatorname{mon}_{a_{1}}\right|_{H_{a_{1}}\left(H_{a_{2}}(m)\right)}
$$

of the LHS should correspond to the automorphism

$$
H_{a_{2}}\left(\left.\operatorname{mon}_{a_{1}}\right|_{H_{a_{1}}(m)}\right)
$$

of the RHS. Again, in the situation arising from abelian categories, this condition is sufficient for the higher compatibilities.

\subsection{Objects in and functors out of $\mathcal{A}^{\otimes Y}$.}

1.8.1. Let us now take $\mathbf{C}=$ DGCat. For a target DG category $\mathcal{C}$, Proposition 1.7 .2 describes what it takes to construct a functor

$$
\mathcal{S}_{Y}: \mathcal{A}^{\otimes Y} \rightarrow \mathcal{C} .
$$

Namely, such a datum is equivalent to a natural transformation between the functors

$$
I \mapsto \mathcal{A}^{\otimes I}
$$

$$
I \mapsto \mathcal{C} \otimes \operatorname{LS}\left(Y^{I}\right)
$$


In other words, this is a data of functors

$$
\mathcal{S}_{I}: \mathcal{A}^{\otimes I} \rightarrow \mathcal{C} \otimes \operatorname{LS}\left(Y^{I}\right), \quad I \in \mathrm{fSet},
$$

that make the following diagrams commute

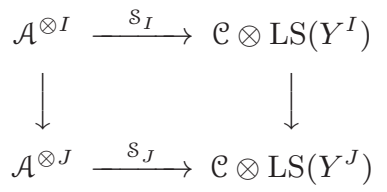

for every $I \rightarrow J$, along with a system of higher compatibilities.

1.8.2. We now make the following observation (which is a special case of Proposition 2.3.9 below applied to the constant diagram $Y \rightarrow$ DGCat $\left.^{\text {SymMon }}\right)$.

Recall the notion of a rigid monoidal category, see Sect. 0.4 .3

Proposition 1.8.3. Let $\mathcal{A}$ be a rigid monoidal $D G$ category. Then $\mathcal{A}^{\otimes Y}$ is also rigid for any $Y \in \mathrm{Spc}$. Moreover, if $\mathcal{A}$ is compactly generated so is $\mathcal{A}^{\otimes Y}$.

Applying [GR1, Chapter 1, Sect. 9.2.1], we obtain:

Corollary 1.8.4. Assume that $\mathcal{A}$ is rigid. Then the functors

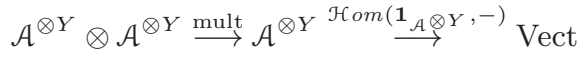

and

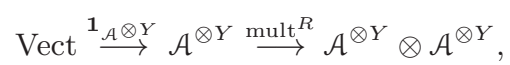

where the first functor in the first line is given by the monoidal operation, and the second functor in the second line is its right adjoint, define an identification

$$
\left(\mathcal{A}^{\otimes Y}\right)^{\vee} \simeq \mathcal{A}^{\otimes Y} .
$$

1.8.5. Taking $\mathcal{C}=$ Vect, and applying Corollary 1.8.4 we obtain that the category

$$
\operatorname{Funct}\left(\mathcal{A}^{\otimes Y}, \mathrm{Vect}\right) \simeq\left(\mathcal{A}^{\otimes Y}\right)^{\vee}
$$

is canonically equivalent to $\mathcal{A}^{\otimes Y}$ itself via the map $r \mapsto \mathcal{H} \circ m_{\mathcal{A} \otimes Y}\left(\mathbf{1}_{\mathcal{A} \otimes Y}, r \otimes-\right)$.

Note that in the description of Sect. 1.8.1 for

$$
\mathcal{S}_{\text {univ }} \in \mathcal{A}^{\otimes Y},
$$

the corresponding functor

$$
\mathcal{S}_{Y}: \mathcal{A}^{\otimes Y} \rightarrow \text { Vect }
$$

is given in terms of (1.29) by the family of functors

$$
\mathcal{S}_{I}: \mathcal{A}^{\otimes I} \rightarrow \operatorname{LS}\left(Y^{I}\right)
$$

that are described as follows:

The datum of (1.31) is equivalent to the map from $\operatorname{Maps}(I, Y) \simeq Y^{I}$ to space of functors

$$
\mathcal{A}^{\otimes I} \rightarrow \text { Vect . }
$$

For a point $\underline{y} \in Y^{I} \simeq \operatorname{Maps}(I, Y)$ and $r \in \mathcal{A}^{\otimes I}$, denote by $r_{\underline{y}}$ the corresponding object of $\mathcal{A}^{\otimes Y}$. By unwinding the definitions, we obtain that (1.32) sends

$$
r \mapsto \mathcal{H}^{\circ} m_{\mathcal{A} \otimes Y}\left(\mathbf{1}_{\mathcal{A} \otimes Y}, r_{\underline{y}} \otimes \mathcal{S}_{\text {univ }}\right) .
$$

1.8.6. In particular, under the correspondence of Sect. 1.8.5, the object

$$
\mathcal{S}_{\emptyset} \in \operatorname{Funct}\left(\mathcal{A}^{\otimes \emptyset}, \operatorname{LS}\left(Y^{\emptyset}\right)\right) \simeq \operatorname{Funct}(\text { Vect, Vect }) \simeq \operatorname{Vect}
$$

identifies with

$$
\mathcal{H} \text { om }\left(\mathbf{1}_{\mathcal{A} \otimes Y}, \mathcal{S}_{\text {univ }}\right) \text {. }
$$




\section{EXCURSIONS}

The goal of this section is to give a (more explicit) description of the category $\mathcal{A}^{\otimes Y}$ in the case when $Y$ is a pointed connected space, and, more substantially, of the endomorphism algebra of its unit object. The latter will be described in terms of what we will call, following V. Lafforgue, "excursion operators".

\subsection{Description of $\mathcal{A}^{\otimes Y}$ via the fundamental group.}

2.1.1. Let $\mathbb{E}_{1}(\mathrm{Spc})$ denote the category of monoid objects in Spc (a.k.a. $\mathbb{E}_{1}$-objects in Spc with respect to the Cartesian monoidal structure). I.e., these are objects of Spc equipped with a binary operation and a unit that satisfy the axioms of monoid up to coherent homotopy.

We have an adjoint pair (see [Lu2, Example 3.1.3.6, Lemma 3.2.2.6 and Prop. 3.2.3.1])

$$
\text { free }_{\mathbb{E}_{1}}: \mathrm{Spc} \rightleftarrows \mathbb{E}_{1}(\mathrm{Spc}): \mathbf{o b l v}_{\mathbb{E}_{1}},
$$

where $\mathbf{o b l v} \mathbb{E}_{\mathbb{E}_{1}}$ preserves sifted colimits and is conservative. Explicitly,

$$
\operatorname{free}_{\mathbb{E}_{1}}(Y)=\sqcup_{n} Y^{n} .
$$

2.1.2. Recall also that the category $\mathbb{E}_{1}(\mathrm{Spc})$ is connected by a pair of mutually adjoint functors to the category of pointed spaces

$$
\begin{gathered}
\mathrm{Spc}_{\mathrm{Ptd}}:=\mathrm{Spc}_{\{*\}}, \\
B: \mathbb{E}_{1}(\mathrm{Spc}) \rightleftarrows \operatorname{Spc}_{\mathrm{Ptd}}: \Omega .
\end{gathered}
$$

This adjunction factors as

$$
\mathbb{E}_{1}(\mathrm{Spc}) \rightleftarrows \mathrm{Spc}_{\mathrm{cnctd}, \mathrm{Ptd}} \rightleftarrows \mathrm{Spc}_{\mathrm{Ptd}},
$$

where $\mathrm{Spc}_{\text {cnctd,Ptd }} \subset \mathrm{Spc}_{\mathrm{Ptd}}$ is the full subcategory of connected pointed spaces.

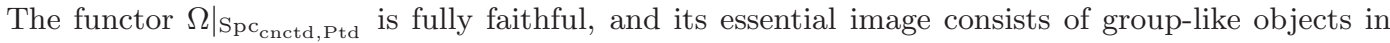
$\mathbb{E}_{1}(\mathrm{Spc})$, i.e., those objects $Y$, for which $\pi_{0}(Y)$, equipped with a monoid structure induced by that on $Y$, is actually a group (see [Lu2, Theorem 5.2.6.10] for an $\infty$-categorical account of this basic fact, which is originally due to Stasheff [St]).

2.1.3. Let FFM denote the full subcategory of $\mathbb{E}_{1}(\mathrm{Spc})$ equal to the essential image of the functor free $_{\mathbb{E}_{1}}$ applied to the full subcategory

$$
\text { fSet } \subset \text { Spc . }
$$

Note that the category FFM is discrete (a.k.a. ordinary). Its objects are the usual free finitely generated monoids, and morphisms are morphisms between such.

2.1.4. The following assertion is probably well-known, but we will supply a proof for completeness:

Proposition 2.1.5. For $Y \in \mathrm{Spc}_{\mathrm{cnctd}, \mathrm{Ptd}}$, the slice category $\mathrm{FFM}_{/ \Omega(Y, y)}$ is sifted, and the natural map

$$
\underset{H \in \mathrm{FFM}_{/ \Omega(Y, y)}}{\operatorname{colim}} B(H) \rightarrow Y
$$

is an isomorphism, where the colimit is taken in the category of pointed spaces.

As an immediate corollary we obtain:

Corollary 2.1.6. For $Y \in \mathrm{Spc}_{\mathrm{cnctd}, \mathrm{Ptd}}$, we have a canonical identification

$$
\mathcal{A}^{\otimes Y} \simeq \underset{H \in \mathrm{FFM}_{/ \Omega(Y, y)}}{\operatorname{colim}} \mathcal{A}^{\otimes B(H)}
$$

Proof. Since the functor

$$
Y \mapsto \mathcal{A}^{\otimes Y}
$$

preserves colimits, we only need to show that the isomorphism (2.2) remains valid if the colimit is understood as taking place in $\mathrm{Spc}$ rather than $\mathrm{Spc}_{\mathrm{Ptd}}$. However, this is always the case any time the index category is contractible, which in our case it is: indeed, it is sifted, and any sifted category is contractible (see [Lu1, Prop. 5.5.8.7]). 
In Sect. 2.4 we will use Corollary 2.1.6 to give an explicit description of the algebra $\operatorname{End}_{\mathcal{A} \otimes Y}\left(\mathbf{1}_{\mathcal{A} \otimes Y}\right)$.

\subsection{Proof of Proposition 2.1.5.}

2.2.1. We will prove the following:

Proposition 2.2.2. For every $H_{0} \in \mathbb{E}_{1}(\mathrm{Spc})$, the slice category $\mathrm{FFM}_{/ H_{0}}$ is sifted, and the map

$$
\underset{H \in \mathrm{FFM}_{/ H_{0}}}{\operatorname{colim}} H \rightarrow H_{0}
$$

is an isomorphism.

Proposition 2.2.2 implies Proposition 2.1.5 take $H_{0}=\Omega(Y, y)$ for $Y \in \mathrm{Spc}_{\mathrm{cnctd}, \mathrm{Ptd}}$, and apply the functor $B$ to 2.3 .

Remark 2.2.3. Instead of monoids in Proposition 2.2.2 we can just as well consider semi-groups; i.e. $\mathbb{E}_{1}$ spaces which are not required to have a unit element. Indeed, by [Lu2, Corollary 5.4.3.6], the forgetful functor from grouplike $\mathbb{E}_{1}$-algebras to $\mathbb{E}_{1}$-semi-groups is fully faithful. A slight adavantage of this is that there are fewer maps between free semi-groups and therefore we obtain a slightly smaller indexing category.

2.2.4. The rest of this subsection is devoted to the proof of Proposition 2.2.2 We claim:

Lemma 2.2.5.

(a) The category FFM has finite coproducts and the functor $\mathrm{FFM} \rightarrow \mathbb{E}_{1}(\mathrm{Spc})$ preserves finite coproducts.

(b) The Yoneda embedding $j: \mathbb{E}_{1}(\mathrm{Spc}) \rightarrow \mathrm{PShv}(\mathrm{FFM})$ is conservative and preserves sifted colimits.

In point (b) of the lemma, for a small category $\mathbf{C}$ (in our case $\mathbf{C}=\mathrm{FFM}$ ), we are using the notation $\operatorname{PShv}(\mathbf{C})$ to denote all functors $\mathbf{C}^{\text {op }} \rightarrow$ Spc.

\subsubsection{Let us show how Lemma 2.2.5 implies Proposition 2.2.2}

By Lemma 2.2.5 and Corollary 1.3.4 the category $\mathrm{FFM}_{/ H_{0}}$ is sifted. Next, since $j$ is conservative it suffices to show that the map

$$
j\left(\operatorname{colim}_{H \in \mathrm{FFM}_{/ H_{0}}} H\right) \rightarrow j\left(H_{0}\right)
$$

is an isomorphism.

Since $j$ preserves sifted colimits, we have to show that the map

$$
\underset{H \in \mathrm{FFM}_{/ H_{0}}}{\operatorname{colim}} j(H) \rightarrow j\left(H_{0}\right)
$$

is an isomorphism. But this follows from the Yoneda Lemma.

$\square[$ Proposition 2.2 .2

2.2.7. We now proceed to the proof of Lemma 2.2.5 First, we state its analog for spaces:

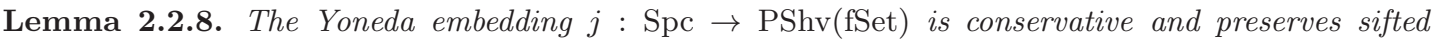
colimits.

Proof. To prove that $j$ preserves sifted colimits, its suffices to show that for every $I \in$ fSet the map $\operatorname{Maps}_{S p c}(I,-)$ preserves sifted colimits. But this is standard (and has been used already in Sect. 1.3.8).

The functor $j$ is conservative, since it has a left inverse

$$
\operatorname{PShv}(\text { fSet }) \rightarrow \operatorname{PShv}(\{*\})=\operatorname{Spc},
$$

induced by the inclusion $\{*\} \rightarrow$ fSet.

Let us now prove Lemma 2.2.5. 
Proof. Point (a) follows from the fact that free $\mathbb{E}_{1}$ is a left adjoint. We now prove point (b).

Consider the functor free $\mathbb{E}_{1}:$ fSet $\rightarrow$ FFM, and the corresponding pullback functor

$$
\text { free }_{\mathbb{E}_{1}}^{*}: \operatorname{PShv}(\mathrm{FFM}) \rightarrow \operatorname{PShv}(\text { fSet }) \text {. }
$$

The functor free $\mathbb{E}_{\mathbb{1}_{1}}^{*}$ preserves all colimits (tautologically), and is conservative, since free $\mathbb{E}_{1}$ is essentially surjective.

Hence, it suffices to show that the composition:

$$
\text { free }_{\mathbb{E}_{1}}^{*} \circ j: \mathbb{E}_{1}(\mathrm{Spc}) \rightarrow \operatorname{PShv}(\mathrm{fSet})
$$

is conservative and preserves sifted colimits. Since free $\mathbb{E}_{1}$ is the left adjoint of $\mathbf{o b l v}_{\mathbb{E}_{1}}$, the above composition factors as

$$
j \circ \mathbf{o b l v}_{\mathbb{E}_{1}}: \mathbb{E}_{1}(\mathrm{Spc}) \rightarrow \mathrm{Spc} \rightarrow \mathrm{PShv}(\mathrm{fSet}) .
$$

Since oblv $\mathbb{E}_{1}$ is conservative and preserves sifted colimits (see [Lu2, Lemma 3.2.2.6 and Prop. 3.2.3.1]), the assertion follows from Lemma 2.2.8

\subsection{Digression: affine functors.}

2.3.1. Let $F: \mathcal{C}_{0} \rightarrow \mathcal{C}$ be a map monoidal categories, which admits a continuous right adjoint as a map of plain DG categories. The functor $F^{R}$ has a natural right-lax monoidal structure; hence the object

$$
F^{R}\left(\mathbf{1}_{\mathcal{C}}\right) \in \mathcal{C}_{0}
$$

has a natural structure of associative algebra in $\mathcal{C}^{0}$ (see [Lu2, Cor. 7.3.2.7]).

Moreover, the functor $F^{R}$ naturally upgrades to a functor

$$
\left(F^{R}\right)^{\mathrm{enh}}: \mathcal{C} \rightarrow F^{R}\left(\mathbf{1}_{\mathcal{C}}\right)-\bmod \left(\mathcal{C}_{0}\right)
$$

Definition 2.3.2. We shall say that $F$ is affine if (2.4) is an equivalence.

For example, if $f: z_{1} \rightarrow z_{2}$ is a quasi-affine map between prestacks, the functor

$$
f^{*}: \mathrm{QCoh}\left(z_{2}\right) \rightarrow \mathrm{QCoh}\left(z_{1}\right)
$$

is affine, see GR1, Chapter 3, Prop. 3.3.3].

2.3.3. Here is a handy criterion of affineness (which follows immediately from the Barr-Beck-Lurie theorem):

Lemma 2.3.4. A functor $F$ is affine if and only if its right adjoint $F^{R}$ has the following properties:

(i) It is continuous;

(ii) It is conservative;

(iii) The right-lax compatibility of $F^{R}$ with the action of $\mathcal{C}_{0}$ is strict.

2.3.5. Let $\mathcal{A}$ be a symmetric monoidal category.

Definition 2.3.6. We shall say that $\mathcal{A}$ has an affine diagonal if the tensor product functor

$$
\mathcal{A} \otimes \mathcal{A} \rightarrow \mathcal{A}
$$

is affine.

For example (assuming that e contains $\mathbb{Q}$ ), for an algebraic group $G$, the category

$$
\operatorname{Rep}(\mathrm{G}) \simeq \mathrm{QCoh}(\mathrm{pt} / \mathrm{G})
$$

has affine diagonal, because the morphism

$$
\mathrm{pt} / \mathrm{G} \rightarrow \mathrm{pt} / \mathrm{G} \times \mathrm{pt} / \mathrm{G}
$$

is affine (here we are using the equivalence (1.21). 
Remark 2.3.7. It is easy to see that a symmetric monoidal category $\mathcal{A}$ is rigid (see Sect. 0.4 .3 for what this means) if and only if it has an affine diagonal and its unit object is compact. This follows immediately from Lemma 2.3 .4 above.

2.3.8. We can use the characterization of rigid symmetric monoidal categories in Remark 2.3.7 to prove that rigid symmetric monoidal categories are stable under colimits:

Proposition 2.3.9. Let $I \rightarrow \mathrm{DGCat}^{\text {SymMon }}$ be a diagram of symmetric monoidal DG categories such that for each $i \in I$, the corresponding category $\mathcal{C}_{i}$ is rigid and for each morphism $i \rightarrow j \in I$, the corresponding functor $\mathcal{C}_{i} \rightarrow \mathcal{C}_{j}$ admits a continuous right adjoint. Then the colimit $\operatorname{colim}_{i \in I} \mathcal{C}_{i}$ is rigid. Moreover, if each $\mathcal{C}_{i}$ is compactly generated, so is $\underset{i \in I}{\operatorname{colim}} \mathcal{C}_{i}$.

In the proof of Proposition 2.3.9, we will use the following basic fact about colimits:

Proposition 2.3.10. Let $\mathbf{C}$ be a category that admits all colimits. Suppose that we have a diagram $F: I \times[1] \rightarrow \mathbf{C}$, where $I$ is a contractible category:

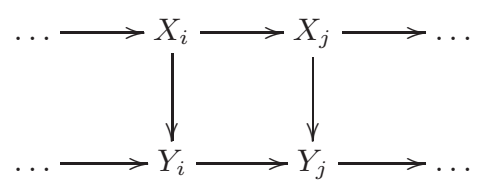

Let $X=\operatorname{colim} X_{i}$. Then the natural map

$$
\underset{i \in I}{\operatorname{colim}} Y_{i} \rightarrow \underset{i \in I}{\operatorname{colim}}\left(X \underset{X_{i}}{\sqcup} Y_{i}\right)
$$

is an isomorphism.

Proof. Since push-outs commute with colimits, we have

$$
\operatorname{colim}_{i \in I}\left(X \underset{X_{i}}{\sqcup} Y_{i}\right) \simeq\left(\operatorname{colim}_{i \in I} X\right) \underset{\substack{\operatorname{colim} \\ i \in I}}{\sqcup}\left(\underset{i \in I}{\operatorname{colim}} Y_{i}\right) .
$$

Now the assertion follows from the fact that

$$
\operatorname{colim}_{i \in I} X \simeq X
$$

since $I$ is contractible, so

$$
\left(\operatorname{colim}_{i \in I} X\right) \underset{\substack{\operatorname{colim} \\ i \in I}}{\sqcup} X_{i}\left(\operatorname{colim}_{i \in I} Y_{i}\right) \simeq X \underset{X}{\sqcup}\left(\operatorname{colim}_{i \in I} Y_{i}\right) \simeq\left(\operatorname{colim}_{i \in I} Y_{i}\right) .
$$

Proof of Proposition 2.3.9. Since all colimits decompose as coproducts and sifted colimits, we can treat each case separately. Suppose $\mathcal{C}_{0}$ and $\mathcal{C}_{1}$ are rigid symmetric monoidal DG categories. Clearly, $\mathcal{C}_{0} \otimes \mathcal{C}_{1}$ (which is the coproduct in DGCat ${ }^{\text {SymMon}}$ ) is also rigid. Moreover, by GR1, Chapter 1, Proposition 7.4.2] it is compactly generated if $\mathcal{C}_{0}$ and $\mathcal{C}_{1}$ are.

Now, suppose that $I$ is sifted. In this case, the underlying DG category of $\mathcal{C}:=\operatorname{colim}_{i \in I} \mathcal{C}_{i}$ is the corresponding colimit in DGCat. In particular, for every $i \in I$, the functor

$$
\operatorname{ins}_{i}: \mathcal{C}_{i} \rightarrow \mathcal{C}
$$

admits a continuous right adjoint and therefore preserves compact objects. It follows, that $\mathbf{1}_{\mathcal{C}} \simeq$ $\operatorname{ins}_{i}\left(\mathbf{1}_{\mathfrak{C}_{i}}\right.$ ) (for any $i \in I$ ) is compact. Moreover, if each $\mathcal{C}_{i}$ is compactly generated, so is $\mathcal{C}$.

It remains to show that $\mathcal{C}$ has affine diagonal. Note that since $I$ is sifted, we have

$$
\underset{i \in I}{\operatorname{colim}}\left(\mathcal{C}_{i} \otimes \mathcal{C}_{i}\right) \simeq \mathcal{C} \otimes \mathcal{C} .
$$

Therefore, since pushouts in DGCat ${ }^{\text {SymMon }}$ are given by tensor products, we have by Proposition 2.3.10

$$
\mathcal{C} \simeq \operatorname{colim}_{i \in I}(\mathcal{C} \otimes \mathcal{C}) \underset{\mathcal{C}_{i} \otimes \mathfrak{e}_{i}}{\otimes} \mathcal{C}_{i}
$$


For each $i \in I$, the tensor product functor $\mathcal{C}_{i} \otimes \mathcal{C}_{i} \rightarrow \mathcal{C}_{i}$ is affine by assumption. Therefore, so is the functor

$$
\mathcal{C} \otimes \mathcal{C} \rightarrow(\mathcal{C} \otimes \mathcal{C}) \underset{\mathcal{C}_{i} \otimes \mathcal{C}_{i}}{\otimes} \mathcal{C}_{i}
$$

The proposition now follows from the following general assertion.

Proposition 2.3.11. Let $\mathrm{C}$ be a symmetric monoidal DG category. The functor

$$
\operatorname{ComAlg}(\mathrm{C}) \rightarrow \mathrm{DGCat}_{\mathrm{e} /}^{\text {SymMon }} \simeq \mathcal{C}-\mathrm{ComAlg}(\text { DGCat })
$$

given by $A \mapsto A-\bmod (\mathrm{C})$ is fully faithful and commutes with colimits.

Proof. The functor admits a right adjoint given by

$$
(\mathcal{C} \rightarrow \mathcal{D}) \mapsto \underline{\operatorname{Hom}}_{\mathcal{D}}\left(\mathbf{1}_{\mathcal{D}}, \mathbf{1}_{\mathcal{D}}\right),
$$

where $\underline{\operatorname{Hom}}_{\mathcal{D}}(-,-)$ denotes internal Hom relative to the right action of $\mathcal{C}$ on $\mathcal{D}$, i.e.,

$$
\operatorname{Hom}_{\mathcal{C}}\left(c, \underline{\operatorname{Hom}}_{\mathcal{D}}\left(d_{1}, d_{2}\right)\right)=\operatorname{Hom}_{\mathcal{D}}\left(d_{1} \otimes c, d_{2}\right) .
$$

The fully faithfulness of the functor in question follows from the isomorphism

$$
\underline{\operatorname{Hom}}_{A-\bmod (\mathcal{C})}\left(\mathbf{1}_{A-\bmod (\mathcal{C})}, \mathbf{1}_{A-\bmod (\mathcal{C})}\right) \simeq A .
$$

\subsection{Endomorphisms of the unit object in $\mathcal{A}^{\otimes Y}$ as a colimit.}

2.4.1. Let us be in the situation of Lemma 1.4.8 Assume that $A$ has an initial object, denoted $a_{0}$. Fix two objects $\mathbf{c}_{a_{0}}^{\prime}, \mathbf{c}_{a_{0}}^{\prime \prime} \in \mathcal{C}_{a_{0}}$ with $\mathbf{c}_{a_{0}}^{\prime}$ compact. Set

$$
\mathbf{c}^{\prime}:=\operatorname{ins}_{a_{0}}\left(\mathbf{c}_{a_{0}}^{\prime}\right), \mathbf{c}^{\prime \prime}:=\operatorname{ins}_{a_{0}}\left(\mathbf{c}_{a_{0}}^{\prime \prime}\right) \text {. }
$$

We can form an $A$-family of objects of Vect

$$
a \mapsto \mathcal{H}_{\mathcal{C}_{a}}\left(F_{a_{0}, a}\left(\mathbf{c}_{a_{0}}^{\prime}\right), F_{a_{0}, a}\left(\mathbf{c}_{a_{0}}^{\prime \prime}\right)\right),
$$

and we have a natural map

$$
\operatorname{colim}_{a} \mathcal{H}_{o m} \mathcal{C}_{a}\left(F_{a_{0}, a}\left(\mathbf{c}_{a_{0}}^{\prime}\right), F_{a_{0}, a}\left(\mathbf{c}_{a_{0}}^{\prime \prime}\right)\right) \rightarrow \mathcal{H}_{\mathcal{C}}\left(\mathbf{c}^{\prime}, \mathbf{c}^{\prime \prime}\right)
$$

2.4.2. It is not difficult to show that when the index category $A$ is filtered, then the map (2.5) is an isomorphism (see [Ro]). However, it is also easy to give an example when the map (2.5) is a not an isomorphism.

However, we claim:

Theorem 2.4.3. Let $\mathcal{A}$ have an affine diagonal. Then the map (2.5) is an isomorphism for the diagram of Corollary 2.1.6.

A consequence of this theorem that will play a role for us in the sequel is the following:

Corollary 2.4.4. Let $\mathcal{A}$ have an affine diagonal, and assume that $\mathbf{1}_{\mathcal{A}} \in \mathcal{A}$ is compact. Then for a connected $Y \in \operatorname{Spc}$ with a marked point $y \in Y$, we have the following expression for $\mathcal{E}_{n} d_{\mathcal{A} \otimes Y}\left(\mathbf{1}_{\mathcal{A} \otimes Y}\right)$ :

$$
\mathcal{E} n d_{\mathcal{A} \otimes Y}\left(\mathbf{1}_{\mathcal{A} \otimes Y}\right) \simeq \underset{H \in \mathrm{FFM} / \Omega(Y, y)}{\operatorname{colim}} \mathcal{E} n d_{\mathcal{A} \otimes B(H)}\left(\mathbf{1}_{\mathcal{A} \otimes B(H)}\right) .
$$

In Sect. 2.7 we will give an explicit description of the algebras $\mathcal{E} n d_{\mathcal{A} \otimes B(H)}\left(\mathbf{1}_{\mathcal{A} \otimes B(H)}\right)$ for $H \in \mathrm{FFM}$, i.e., for $H$ of the form free $\mathbb{E}_{1}(I)$ for $I \in$ fSet.

The next two subsections are devoted to the proof of Theorem 2.4 .3 
2.4.5. We will prove Theorem 2.4.3 in the following general context:

Proposition 2.4.6. Assume that in the situation of (2.5) the functor

$$
a \mapsto \mathcal{C}_{a}
$$

comes from a functor $A \rightarrow \mathrm{DGCat}^{\mathrm{Mon}}$. Assume that $A$ is sifted and all the functors $\mathcal{C}_{a_{0}} \rightarrow \mathcal{C}_{a}$ are affine. Then the map (2.5) is an isomorphism.

\subsection{Proof of Proposition 2.4.6.}

2.5.1. The assumption that the functors $\mathcal{C}_{a_{0}} \rightarrow \mathcal{C}_{a}$ are affine implies that the assignment

$$
a \mapsto \mathcal{C}_{a}
$$

viewed as a functor

$$
A \rightarrow \text { DGCat }
$$

canonically factors as

$$
A \mapsto \operatorname{AssocAlg}\left(\mathcal{C}_{a_{0}}\right) \rightarrow \text { DGCat }
$$

where:

-the first arrow is the functor $a \mapsto F_{a_{0}, a}^{R}\left(\mathbf{1}_{\mathcal{C}_{a}}\right)$;

-the second arrow is the functor

$$
R \mapsto R-\bmod \left(\mathcal{C}_{0}\right), \quad\left(R_{1} \rightarrow R_{2}\right) \rightsquigarrow\left(M \mapsto R_{2} \underset{R_{1}}{\otimes} M\right) .
$$

In particular, we obtain an $A$-diagram

$$
a \mapsto R_{a} \in \operatorname{AssocAlg}\left(\mathcal{C}_{a_{0}}\right) .
$$

Set

$$
R:=\underset{a}{\operatorname{colim}} R_{a}
$$

where the colimit is taken in $\operatorname{Assoc} \operatorname{Alg}\left(\mathcal{C}_{a_{0}}\right)$.

We have a naturally defined functor

$$
\underset{a \in A}{\operatorname{colim}} R_{a}-\bmod \left(\bigodot_{a_{0}}\right) \rightarrow R-\bmod \left(\bigodot_{a_{0}}\right) .
$$

Lemma 2.5.2. The functor (2.6) is an equivalence.

The proof of the lemma is given below.

2.5.3. Thus, returning to the proof of Proposition 2.4.6 we need to show that for $M_{0}^{\prime}, M_{0}^{\prime \prime} \in \mathcal{C}_{a_{0}}$ with $M_{0}^{\prime}$ compact, the map

$$
\underset{a \in A}{\operatorname{colim}} \operatorname{Maps}_{R_{a}-\bmod \left(\mathcal{C}_{a_{0}}\right)}\left(R_{a} \otimes M_{0}^{\prime}, R_{a} \otimes M_{0}^{\prime \prime}\right) \rightarrow \operatorname{Maps}_{R-\bmod \left(\mathcal{C}_{a_{0}}\right)}\left(R \otimes M_{0}^{\prime}, R \otimes M_{0}^{\prime \prime}\right)
$$

is an isomorphism. We rewrite the LHS as

$$
\operatorname{colim}_{a \in A} \operatorname{Maps}_{\mathfrak{C}_{a_{0}}}\left(M_{0}^{\prime}, \text { oblv }_{\text {Assoc }}\left(R_{a}\right) \otimes M_{0}^{\prime \prime}\right),
$$

and the RHS as

$$
\operatorname{Maps}_{\mathfrak{C}_{a_{0}}}\left(M_{0}^{\prime}, \text { oblv }_{\text {Assoc }}(R) \otimes M_{0}^{\prime \prime}\right) .
$$

Since, $M_{0}^{\prime}$ was assumed compact, we rewrite the LHS further as

$$
\operatorname{Maps}_{\mathfrak{C}_{a_{0}}}\left(M_{0}^{\prime}, \operatorname{colim}_{a \in A} \operatorname{oblv}_{\mathrm{Assoc}}\left(R_{a}\right) \otimes M_{0}^{\prime \prime}\right) \simeq \operatorname{Maps}_{\mathfrak{C}_{a_{0}}}\left(M_{0}^{\prime},\left(\operatorname{colim}_{a \in A} \mathbf{o b l v}_{\mathrm{Assoc}}\left(R_{a}\right)\right) \otimes M_{0}^{\prime \prime}\right) .
$$

Now, the assumption that $A$ is sifted implies that

$$
\operatorname{colim}_{a \in A} \operatorname{oblv}_{\text {Assoc }}\left(R_{a}\right) \simeq \operatorname{oblv}_{\text {Assoc }}\left(\operatorname{colim}_{a \in A} R_{a}\right)=\operatorname{oblv}_{\text {Assoc }}(R),
$$

and the assertion follows. 
2.5.4. First proof of Lemma 2.5.2. Recall the equivalence of Lemma1.4.8 The corresponding category $\widetilde{\mathcal{C}}$ is the limit

$$
\lim _{a \in A} R_{a}-\bmod \left(\mathcal{C}_{a_{0}}\right)
$$

where for $a_{1} \rightarrow a_{2}$, the corresponding functor

$$
R_{a_{2}}-\bmod \left(\mathcal{C}_{a_{0}}\right) \rightarrow R_{a_{1}} \bmod \left(\mathcal{C}_{a_{0}}\right)
$$

is given by restriction along $R_{a_{1}} \rightarrow R_{a_{2}}$.

In terms of the equivalence

$$
\mathcal{e} \simeq \widetilde{\mathcal{C}}
$$

the functor right adjoint to (2.6), viewed as a functor

$$
R-\bmod \left(\complement_{a_{0}}\right) \rightarrow \lim _{a \in A} R_{a}-\bmod \left(\complement_{a_{0}}\right),
$$

is given by restrictions along $R_{a} \rightarrow R$.

Hence, Lemma 2.5 .2 is equivalent to the following statement:

Lemma 2.5.5. Let $\mathcal{C}$ be a monoidal category, in which the monoidal operation is compatible with colimits in each variable. Then for a sifted colimit diagram of algebras in $\mathrm{C}$,

$$
\underset{a}{\operatorname{colim}} R_{a} \simeq R
$$

the functor

$$
R-\bmod (\mathcal{C}) \rightarrow \lim _{a} R_{a}-\bmod (\mathcal{C})
$$

is an equivalence.

Proof of Lemma 2.5.5. Note that functor (2.7) is compatible with the natural forgetful functors of both sides to $\mathcal{C}$. We first show that (2.7) induces an equivalence between the fiber of both categories over a given object of $\mathcal{C}$.

For a pair of objects $M_{1}, M_{2} \in \mathcal{C}$, let $\underline{\operatorname{Hom}}\left(M_{1}, M_{2}\right) \in \mathcal{C}$ be their internal Hom, i.e.,

$$
\operatorname{Maps}_{\mathcal{C}}\left(\mathbf{c}, \underline{\operatorname{Hom}}\left(M_{1}, M_{2}\right)\right) \simeq \operatorname{Maps}\left(\mathbf{c} \otimes M_{1}, M_{2}\right)
$$

(this object exists by the Brown representability theorem, Lu1, Prop. 5.5.2.2]).

For $M_{1}=M_{2}=M$, the object $\underline{\operatorname{End}}(M):=\underline{\operatorname{Hom}}(M, M)$ has a structure of associative algebra. For $R^{\prime} \in \operatorname{AssocAlg}(\mathcal{C})$, the datum of action of $R^{\prime}$ on $M$ is equivalent to a map

$$
R^{\prime} \rightarrow \underline{\operatorname{End}}(M)
$$

in $\operatorname{Assoc} \operatorname{Alg}(\mathcal{C})$ (see [Lu2, Cor. 4.7.1.40]).

Hence, the datum of structure of $R$-module on $M$ is equivalent to that of a homomorphism

$$
R \rightarrow \underline{\operatorname{End}}(M)
$$

and a compatible system of data of $R_{a}$-modules on $M$ is equivalent to that of a point in

$$
\lim _{\mathrm{a}} \operatorname{Maps}_{\mathrm{AssocAlg}(\mathcal{C})}\left(R_{a}, \underline{\operatorname{End}}(M)\right) \text {. }
$$

We rewrite the latter as

$$
\operatorname{Maps}_{\operatorname{AssocAlg}(\mathcal{e})}\left(\underset{\mathrm{a}}{\operatorname{colim}} R_{a}, \underline{\operatorname{End}}(M)\right),
$$

which is by definition

implying the assertion.

$$
\operatorname{Maps}_{\operatorname{AssocAlg}(\mathcal{C})}(R, \underline{\operatorname{End}}(M))
$$

The equivalence of fibers of the two sides of (2.7) over a given $M$ implies that (2.7) induces an equivalence of the underlying groupoids. To show that (2.7) is an equivalence of categories, it remains to show that for $M_{1}, M_{2} \in R$-mod, the map

$$
\mathcal{H o m}_{R-\bmod (\mathfrak{e})}\left(M_{1}, M_{2}\right) \rightarrow \lim _{a \in A} \mathcal{H} \mathcal{H o m}_{R_{a}-\bmod (\mathfrak{e})}\left(M_{1}, M_{2}\right)
$$


is an isomorphism.

Note that for a given $R^{\prime} \in \operatorname{Assoc} A \lg (\mathcal{C})$, and $M_{1}^{\prime}, M_{2}^{\prime} \in R^{\prime}$-mod(C), the object

$$
\mathcal{H}_{\mathrm{om}_{R^{\prime}-\bmod (\mathcal{C})}}\left(M_{1}^{\prime}, M_{2}^{\prime}\right)
$$

is calculated as the limit (a.k.a. totalization) of the co-Bar complex, whose terms are

$$
\mathcal{H}_{\mathrm{O}} \mathrm{m}_{\mathcal{C}}\left(\operatorname{oblv}_{\mathrm{Assoc}}\left(R^{\prime}\right)^{\otimes n} \otimes M_{1}^{\prime}, M_{2}^{\prime}\right) \text {. }
$$

Note that, since $A$ is sifted, for each $n$, the map

$$
\mathcal{H}_{\mathrm{Com}}\left(\operatorname{oblv}_{\mathrm{Assoc}}(R)^{\otimes n} \otimes M_{1}^{\prime}, M_{2}^{\prime}\right) \rightarrow \lim _{a \in A} \mathcal{H}_{\mathrm{Com}}\left(\operatorname{oblv}_{\mathrm{Assoc}}\left(R_{a}\right)^{\otimes n} \otimes M_{1}, M_{2}\right)
$$

is an isomorphism.

Interchanging the order of $\lim _{a \in A}$ and the totalization we obtain that 2.8 is an isomorphism.

2.5.6. Second proof of Lemma 2.5.2. In fact, Lemma 2.5.2 is a corollary of the following more general assertion (see Corollary 2.5.8 below):

Lemma 2.5.7. Let $\mathrm{C}$ be a monoidal $D G$ category. Then the functor

$$
\operatorname{AssocAlg}(\mathcal{C}) \rightarrow\left(\mathcal{C}-\bmod ^{r}(\text { DGCat })\right)_{e}, \quad R \mapsto R-\bmod (\mathcal{C})
$$

is fully faithful and commutes with colimit $\$$.

Proof. Same as the proof of Proposition 2.3.11

Corollary 2.5.8. Let $\mathcal{C}$ be a monoidal DG category. Then the functor

$$
\operatorname{AssocAlg}(\mathcal{C}) \rightarrow \text { DGCat }, \quad R \mapsto R-\bmod (\mathcal{C})
$$

commutes with colimits over contractible index categories.

Proof. Follows from the fact that the forgetful functor

$$
\text { e-mod } \bmod ^{r}(\text { Gat }) \rightarrow \text { DGCat }
$$

commutes with colimits.

\subsection{Applying the paradigm.}

2.6.1. In order to prove Theorem 2.4.3, it suffices to show that it fits into the paradigm of Proposition 2.4.6. Hence, it suffices to prove the following:

Proposition 2.6.2. Let $\mathcal{A}$ have an affine diagonal. Then for a finite set $I$, the functor

$$
\mathcal{A}=\mathcal{A}^{\otimes\{*\}} \simeq \mathcal{A}^{\otimes B\left(\text { free }_{\mathbb{E}_{1}}(\emptyset)\right)} \rightarrow \mathcal{A}^{\otimes B\left(\text { free }_{\mathbb{E}_{1}}(I)\right)}
$$

is affine.

In the process of proving this proposition, we will describe what the category $\mathcal{A}^{\otimes B\left(\text { free }_{\mathbb{E}_{1}}(I)\right)}$ looks like.

Remark 2.6.3. Using the argument in the proof of Theorem 1.6.8 one can strengthen the assertion of Proposition 2.6.2 as follows: for a map of spaces $Y_{1} \rightarrow Y_{2}$ which is surjective on $\pi_{0}$, the resulting map

$$
\mathcal{A}^{Y_{1}} \rightarrow \mathcal{A}^{Y_{2}}
$$

is affine.

\footnotetext{
${ }^{9}$ In the above formula, the superscript " $r$ " stands for "right modules".
} 
2.6.4. Since both functors $B(-)$ and free $\mathbb{E}_{1}$ are left adjoints, we have:

$$
B\left(\text { free }_{\mathbb{E}_{1}}(I)\right) \simeq \underset{I}{\vee} B\left(\text { free }_{\mathbb{E}_{1}}(\{*\})\right),
$$

where $\vee$ means push-out with respect to the common base point. Since pushouts in DGCat ${ }^{\text {SymMon }}$ are tensor products, we obtain:

$$
\mathcal{A}^{\otimes B\left(\text { free }_{\mathbb{E}_{1}}(I)\right)} \simeq \underbrace{\mathcal{A}_{\mathcal{A}}^{\otimes B\left(\text { free }_{\mathbb{E}_{1}}(\{*\})\right)} \otimes_{\mathcal{A}} \ldots \mathcal{A}^{\otimes B\left(\text { free }_{\mathbb{E}_{1}}(\{*\})\right)} .}_{I \text {-fold product }}
$$

Hence, it suffices to show that $\mathcal{A}^{\otimes B\left(\text { free }_{\mathbb{E}_{1}}(\{*\})\right)}$ is affine over $\mathcal{A}$ : indeed, for a pair of commutative algebras $A_{1}$ and $A_{2}$ in $\mathcal{A}$, we have

$$
A_{1}-\bmod (\mathcal{A}) \underset{\mathcal{A}}{\otimes} A_{2}-\bmod (\mathcal{A}) \simeq\left(A_{1} \otimes A_{2}\right)-\bmod (\mathcal{A})
$$

2.6.5. Note that $B\left(\right.$ free $\left._{\mathbb{E}_{1}}(\{*\})\right) \simeq S^{1}$. Indeed, by adjunction

$$
\begin{aligned}
\operatorname{Maps}_{\mathrm{Spc}_{\mathrm{Ptd}}}\left(B\left(\operatorname{free}_{\mathbb{E}_{1}}(\{*\})\right), Y\right) \simeq \operatorname{Maps}_{\mathbb{E}_{1}(\mathrm{Spc})}\left(\text { free }_{\mathbb{E}_{1}}(\{*\}), \Omega(Y, y)\right) \simeq & \\
& \simeq \operatorname{Maps}_{\mathrm{Spc}}(\{*\}, \Omega(Y, y)) \simeq \Omega(Y, y) \simeq \operatorname{Maps}_{\operatorname{Spc}_{\mathrm{Ptd}}}\left(S^{1}, Y\right) .
\end{aligned}
$$

2.6.6. Write $S^{1}$ as a push-out

$$
\{*\} \underset{\{*\} \sqcup\{*\}}{\sqcup}\{*\} .
$$

Hence,

$$
\mathcal{A}^{\otimes B\left(\text { free }_{\mathbb{E}_{1}}(\{*\})\right)} \simeq \mathcal{A}^{\otimes S^{1}} \simeq \mathcal{A} \underset{\mathcal{A} \otimes \mathcal{A}}{\otimes} \mathcal{A}
$$

2.6.7. Hence, it remains to show that if $\mathcal{A}$ has an affine diagonal, then the functor

$$
\mathcal{A} \rightarrow \mathcal{A} \underset{\mathcal{A} \otimes \mathcal{A}}{\otimes} \mathcal{A}
$$

is affine.

Note that the above functor is obtained by base change $\mathcal{A} \underset{\mathcal{A} \otimes \mathcal{A}}{\otimes}-$ from the functor $\mathcal{A} \otimes \mathcal{A} \rightarrow \mathcal{A}$. Hence, it suffices to prove the following assertion:

Lemma 2.6.8. Let $\mathcal{A}_{0} \rightarrow \mathcal{A}$ be an affine functor between symmetric monoidal categories. Then for any diagram of symmetric monoidal categories

$$
\mathcal{B}^{\prime} \leftarrow \mathcal{B} \rightarrow \mathcal{A}_{0},
$$

the resulting functor

$$
\mathcal{B}^{\prime} \underset{\mathcal{B}}{\otimes} \mathcal{A}_{0} \rightarrow \mathcal{B}^{\prime} \underset{\mathcal{B}}{\otimes} \mathcal{A}
$$

is also affine.

Proof. Follows from the fact that for $R \in \operatorname{Assoc} \operatorname{Alg}\left(\mathcal{A}_{0}\right)$, we have

$$
\mathcal{B}^{\prime} \underset{\mathcal{B}}{\otimes} R-\bmod \left(\mathcal{A}_{0}\right) \simeq R^{\prime}-\bmod \left(\mathcal{B}^{\prime} \underset{\mathcal{B}}{\otimes} \mathcal{A}_{0}\right),
$$

where $R^{\prime}$ denotes the image of $R$ under

$$
\mathcal{A}_{0} \rightarrow \mathcal{B}^{\prime} \underset{\mathcal{B}}{\otimes} \mathcal{A}_{0}
$$

Indeed, the adjunction

$$
\left(\mathrm{id} \otimes \text { ind }_{R}\right): \mathcal{B}^{\prime} \underset{\mathcal{B}}{\otimes} \mathcal{A}_{0} \rightleftarrows \mathcal{B}^{\prime} \underset{\mathcal{B}}{\otimes} R-\bmod \left(\mathcal{A}_{0}\right):\left(\mathrm{id} \otimes \mathbf{o b l v}_{R}\right)
$$

satisfies the assumption of the Barr-Beck-Lurie theorem (see [Lu2, Theorem 4.7.3.5]) and hence is monadic, with the monad given by $R^{\prime} \otimes-$.

\subsection{Endomorphisms of the unit, term-wise.}


2.7.1. For a finite set $I$, let $I_{+}$denote the pointed finite set

$$
I_{+}=I \sqcup\{*\} .
$$

Note that we have a canonical identification of pointed spaces

$$
B\left(\text { free }_{\mathbb{E}_{1}}(I)\right) \simeq\{*\} \underset{I_{+}}{\sqcup}\{*\}=: \Sigma\left(I_{+}\right) .
$$

Indeed, by adjunction, for a pointed space $(Y, y)$, the following five pieces of data are equivalent:

(i) A map of pointed spaces $\Sigma\left(I_{+}\right) \rightarrow(Y, y)$;

(ii) A map of pointed spaces $\left(I_{+}, *\right) \rightarrow \Omega(Y, y)$;

(iii) A map of spaces $I \rightarrow \Omega(Y, y)$;

(iv) A map of $\mathbb{E}_{1}$-objects free $\mathbb{E}_{1}(I) \rightarrow \Omega(Y, y)$;

(v) A map of pointed spaces $B\left(\right.$ free $\left._{\mathbb{E}_{1}}(I)\right) \rightarrow(Y, y)$.

2.7.2. In what follows, for $\gamma^{I} \in \operatorname{Maps}(I, \Omega(Y, y))$, we let $\gamma^{I_{+}}$denote the corresponding pointed map $I_{+} \rightarrow \Omega(Y, y)$, i.e.,

$$
\gamma^{I+}=\left(\gamma^{I}, \gamma_{\text {triv }}\right)
$$

Thus, for each $\gamma^{I}$ we obtain a map $\Sigma\left(I_{+}\right) \rightarrow Y$ and the corresponding map of algebras, to be denoted

$$
\gamma^{I_{+}}: \operatorname{End}_{\mathcal{A}^{\otimes \Sigma\left(I_{+}\right)}}\left(\mathbf{1}_{\mathcal{A}} \otimes \Sigma\left(I_{+}\right)\right) \rightarrow \operatorname{End}_{\mathcal{A} \otimes Y}\left(\mathbf{1}_{\mathcal{A} \otimes Y}\right) .
$$

According to Corollary 2.4.4 the resulting map from the colimit of the terms

$$
\operatorname{End}_{\mathcal{A}^{\otimes \Sigma\left(I_{+}\right)}}\left(\mathbf{1}_{\mathcal{A}^{\otimes \Sigma\left(I_{+}\right)}}\right)
$$

to $\operatorname{End}_{\mathcal{A} \otimes Y}\left(\mathbf{1}_{\mathcal{A} \otimes Y}\right)$, is an isomorphism, provided that $\mathcal{A}$ has an affine diagonal.

In this subsection we will describe the terms (2.10) more explicitly, assuming that $\mathcal{A}$ is rigid (note that according to Remark 2.3.7 a rigid symmetric monoidal category automatically has an affine diagonal).

2.7.3. For a (not necessarily pointed) finite set $J$, consider commutative diagram

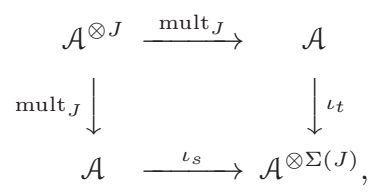

where the two arrows $\iota_{s}, \iota_{t}: \mathcal{A} \rightarrow \mathcal{A}^{\otimes \Sigma(J)}$ correspond to the two maps

$$
\{*\} \rightrightarrows \Sigma(J) \text {. }
$$

Note that since the functor (1.3) preserves colimits, (2.11) is in fact a push-out square in DGCat ${ }^{\text {SymMon }}$. Base change defines a natural transformation

$$
\mathrm{BC}_{J}: \operatorname{mult}_{J} \circ\left(\operatorname{mult}_{J}\right)^{R} \rightarrow \iota_{s}^{R} \circ \iota_{t} .
$$

Lemma 2.7.4. Assume that $\mathcal{A}$ is rigid. Then the natural transformation $\mathrm{BC}_{J}$ is an isomorphism.

Proof. We will prove the assertion more generally for a push-out diagram of rigid symmetric monoidal categories

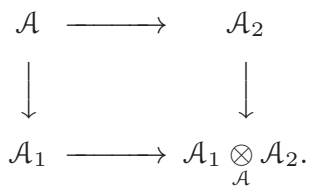


Indeed, we rewrite the latter diagram as

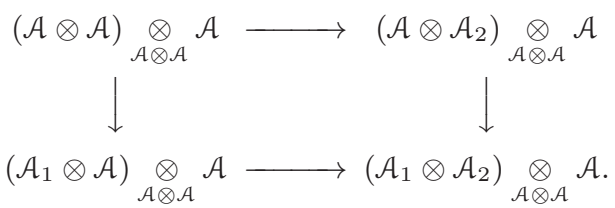

Now, the assertion follows using [GR1, Chapter 1, Lemma 9.3.6] from the base change property of the diagram

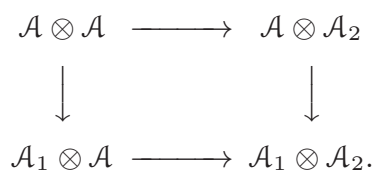

Namely, the assertion of loc.cit. says that the right adjoint of a functor between module categories over a rigid monoidal category, which is a priori only right-lax compatible with the action, is actually strictly compatible. Hence, the resulting (commutative) diagram

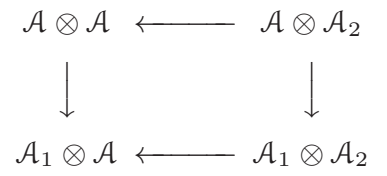

takes place in $(\mathcal{A} \otimes \mathcal{A})$ - $\bmod$, and hence stays commutative after applying $-\underset{\mathcal{A} \otimes \mathcal{A}}{\otimes} \mathcal{A}$.

\subsubsection{Note that}

$$
\iota_{s}\left(\mathbf{1}_{\mathcal{A}}\right) \simeq \mathbf{1}_{\mathcal{A} \otimes \Sigma(J)} \simeq \iota_{t}\left(\mathbf{1}_{\mathcal{A}}\right)
$$

Hence,

$$
\mathcal{H}_{\operatorname{com}}\left(\mathbf{1}_{\mathcal{A}}, \iota_{s}^{R} \circ \iota_{t}\left(\mathbf{1}_{\mathcal{A}}\right)\right) \simeq \mathcal{E} n d_{\mathcal{A} \otimes \Sigma(J)}\left(\mathbf{1}_{\mathcal{A} \otimes \Sigma(J)}\right) .
$$

Therefore, as a corollary of Lemma 2.7.4 we obtain:

Corollary 2.7.6. For $\mathcal{A}$ rigid, we have a canonical isomorphism (in Vect)

$$
\mathcal{H}_{\operatorname{com}}\left(\mathbf{1}_{\mathcal{A}}, \text { mult }_{J} \circ \operatorname{mult}_{J}^{R}\left(\mathbf{1}_{\mathcal{A}}\right)\right) \rightarrow \mathcal{E} n d_{\mathcal{A} \otimes \Sigma(J)}\left(\mathbf{1}_{\mathcal{A} \otimes \Sigma(J)}\right) .
$$

2.7.7. Note that the functor mult ${ }_{J}$ is symmetric monoidal. Hence, the functor mult ${ }_{J}^{R}$ has a natural right-lax symmetric monoidal structure. In particular, the object

$$
\left(\operatorname{mult}_{J}\right)^{R}\left(\mathbf{1}_{\mathcal{A}}\right)
$$

has a natural structure of commutative algebra in $\mathcal{A}^{\otimes J}$, and hence

$$
\text { mult }_{J} \circ\left(\text { mult }_{J}\right)^{R}\left(\mathbf{1}_{\mathcal{A}}\right)
$$

has a natural structure of commutative algebra in $\mathcal{A}$.

Hence,

$$
\operatorname{Maps}_{\mathcal{A}}\left(\mathbf{1}_{\mathcal{A}}, \operatorname{mult}_{J} \circ\left(\operatorname{mult}_{J}\right)^{R}\left(\mathbf{1}_{\mathcal{A}}\right)\right)
$$

acquires a structure of commutative algebra (in Vect).

2.7.8. For a pair of elements $\xi_{1}, \xi_{2} \in \operatorname{Maps}_{\mathcal{A}}\left(\mathbf{1}_{\mathcal{A}}, \operatorname{mult}_{J} \circ \operatorname{mult}_{J}^{R}\left(\mathbf{1}_{\mathcal{A}}\right)\right)$, let us denote by $\xi_{1} * \xi_{2}$ their product in $\operatorname{Maps}_{\mathcal{A}}\left(\mathbf{1}_{\mathcal{A}}\right.$, mult $\left._{J} \circ \operatorname{mult}_{J}^{R}\left(\mathbf{1}_{\mathcal{A}}\right)\right)$.

Explicitly, $\xi_{1} * \xi_{2}$ is given by the composition

$$
\begin{aligned}
\mathbf{1}_{\mathcal{A}} \simeq \mathbf{1}_{\mathcal{A}} \otimes & \mathbf{1}_{\mathcal{A}} \stackrel{\xi_{1} \otimes \xi_{2}}{\longrightarrow} \operatorname{mult}_{J} \circ \operatorname{mult}_{J}^{R}\left(\mathbf{1}_{\mathcal{A}}\right) \otimes \operatorname{mult}_{J} \circ \operatorname{mult}_{J}^{R}\left(\mathbf{1}_{\mathcal{A}}\right) \simeq \\
& \simeq \operatorname{mult}_{J}\left(\operatorname{mult}_{J}^{R}\left(\mathbf{1}_{\mathcal{A}}\right) \otimes \operatorname{mult}_{J}^{R}\left(\mathbf{1}_{\mathcal{A}}\right)\right) \rightarrow \operatorname{mult}_{J} \circ \operatorname{mult}_{J}^{R}\left(\mathbf{1}_{\mathcal{A}} \otimes \mathbf{1}_{\mathcal{A}}\right) \simeq \operatorname{mult}_{J} \circ \operatorname{mult}_{J}^{R}\left(\mathbf{1}_{\mathcal{A}}\right) .
\end{aligned}
$$


2.7.9. We now claim:

Lemma 2.7.10. The isomorphism of Corollary 2.7.6 respects the algebra structures on the two sides.

Proof. Since (2.11) is a commutative diagram of symmetric monoidal DG categories, the base change morphism 2.12) respects the right-lax symmetric monoidal structures on the two sides. Hence, the induced morphism

$$
\mathcal{H}_{\operatorname{com}}\left(\mathbf{1}_{\mathcal{A}}, \text { mult }_{J} \circ \operatorname{mult}_{J}^{R}\left(\mathbf{1}_{\mathcal{A}}\right)\right) \rightarrow \mathcal{H}_{\operatorname{com}}\left(\mathbf{1}_{\mathcal{A}}, \iota_{s}^{R} \circ \iota_{r}\left(\mathbf{1}_{\mathcal{A}}\right)\right) \simeq \mathcal{E} n d_{\mathcal{A} \otimes \Sigma(J)}\left(\mathbf{1}_{\mathcal{A} \otimes \Sigma(J)}\right)
$$

respects the commutative algebra structures.

Finally, the resulting algebra structure on $\mathcal{E} n d_{\mathcal{A} \otimes \Sigma(J)}\left(\mathbf{1}_{\mathcal{A} \otimes \Sigma(J)}\right)$ equals one given by the composition, by the Eckmann-Hilton argument.

2.7.11. In what follows for

$$
\xi \in \operatorname{Maps}_{\mathcal{A}}\left(\mathbf{1}_{\mathcal{A}}, \operatorname{mult}_{J} \circ \operatorname{mult}_{J}^{R}\left(\mathbf{1}_{\mathcal{A}}\right)\right)
$$

we let

$$
E_{\xi} \in \mathcal{E} n d_{\mathcal{A} \otimes \Sigma(J)}\left(\mathbf{1}_{\mathcal{A} \otimes \Sigma(J)}\right)
$$

denote the corresponding element.

Let us describe explicitly the product operation on the elements $E_{\xi}$ of (2.14). By Lemma 2.7.10 we have

$$
E_{\xi_{1}} \cdot E_{\xi_{2}}=E_{\xi_{1} * \xi_{2}}
$$

where $\xi_{1} * \xi_{2}$ is the product of $\xi_{1}$ and $\xi_{2}$ in $\operatorname{Maps}_{\mathcal{A}}\left(\mathbf{1}_{\mathcal{A}}, \operatorname{mult}_{J} \circ \operatorname{mult}{ }_{J}^{R}\left(\mathbf{1}_{\mathcal{A}}\right)\right)$, which is in turn described explicitly in Sect. 2.7.8

\subsection{Action on a module via excursions.}

2.8.1. Let us be given a functor of DG categories

$$
\mathcal{S}_{Y}: \mathcal{A}^{\otimes Y} \rightarrow \mathcal{C} .
$$

In particular, the algebra $\mathcal{E} n d_{\mathcal{A} \otimes Y}\left(\mathbf{1}_{\mathcal{A} \otimes Y}\right)$ acts on the object $\mathcal{S}_{Y}\left(\mathbf{1}_{\mathcal{A} \otimes Y}\right)$.

Recall (see Sect. 2.7.2) that given a map

$$
\gamma^{I}: I \rightarrow \Omega(Y, y)
$$

we have a map

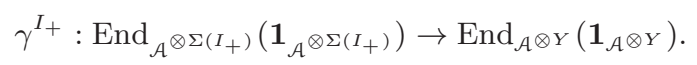

Recall that to an element

$$
\xi \in \operatorname{Maps}_{\mathcal{A}}\left(\mathbf{1}_{\mathcal{A}}, \text { mult }_{J_{+}} \circ\left(\operatorname{mult}_{I_{+}}\right)^{R}\left(\mathbf{1}_{\mathcal{A}}\right)\right)
$$

we associated an element

$$
E_{\xi} \in \mathcal{E} n d_{\mathcal{A}^{\otimes \Sigma\left(I_{+}\right)}}\left(\mathbf{1}_{\mathcal{A}^{\otimes \Sigma\left(J_{+}\right)}}\right) .
$$

In this subsection we will give an explicit formula for the action of the element

$$
\gamma^{I+}\left(E_{\xi}\right) \in \mathcal{E} n d_{\mathcal{A} \otimes Y}\left(\mathbf{1}_{\mathcal{A} \otimes Y}\right)
$$

on $\mathcal{S}_{Y}\left(\mathbf{1}_{\mathcal{A} \otimes Y}\right)$.

We will do so in a slightly more general context: instead of the pointed finite set $I_{+}$we will consider a non-pointed one. 
2.8.2. For an object $Y^{\prime} \in \mathrm{Spc}$ and a point $y^{\prime} \in Y^{\prime}$ let $\mathrm{ev}_{y^{\prime}}$ denote the restriction functor

$$
\mathrm{LS}\left(Y^{\prime}\right) \rightarrow \text { Vect }
$$

corresponding to

$$
\{*\} \stackrel{y^{\prime}}{\rightarrow} Y^{\prime} .
$$

Given two points $y_{1}^{\prime}$ and $y_{2}^{\prime}$ and a path $\gamma^{\prime}$ between them, we have a natural transformation

$$
\operatorname{mon}_{\gamma^{\prime}}: \mathrm{ev}_{y_{1}^{\prime}} \rightarrow \mathrm{ev}_{y_{2}^{\prime}},
$$

given by "parallel transport" along $\gamma$. Here is the formal construction:

By definition, a path $\gamma^{\prime}$ is a point of $\{*\} \underset{Y^{\prime}}{\times}\{*\}$, where the two maps

$$
\{*\} \rightrightarrows Y^{\prime}
$$

are given by $y_{1}^{\prime}$ and $y_{2}^{\prime}$, respectively.

Restriction on $\operatorname{LS}(-)$ along the maps in the commutative square

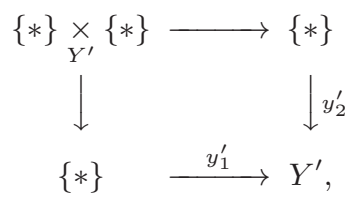

followed by restriction along

$$
\{*\} \stackrel{\gamma^{\prime}}{\rightarrow}\{*\} \underset{Y^{\prime}}{\times}\{*\}
$$

defines the desired isomorphism of functors 2.15).

2.8.3. Ley $y_{1}$ and $y_{2}$ be two points of $Y$. Let $J$ be a finite set, and let us be given a $J$-tuple $\gamma^{J}$ of paths from $y_{1}$ to $y_{2}$. We can consider the points $y_{1}^{J}, y_{2}^{J} \in Y^{J}$ and regard $\gamma^{J}$ as a path from $y_{1}^{J}$ to $y_{2}^{J}$.

Fix a point

$$
\xi \in \operatorname{Maps}_{\mathcal{A}}\left(\mathbf{1}_{\mathcal{A}}, \operatorname{mult}_{J} \circ \operatorname{mult}_{J}^{R}\left(\mathbf{1}_{\mathcal{A}}\right)\right)
$$

Recall that according to Proposition 1.7.2 the datum of a functor $\mathcal{S}_{Y}$ is equivalent to the datum of a collection of functors

$$
\mathcal{S}_{I}: \mathcal{A}^{\otimes I} \rightarrow \mathcal{C} \otimes \operatorname{LS}\left(Y^{I}\right)
$$

which depends functorially on $I \in$ fSet.

Define the excursion operator $\operatorname{Exc}_{\mathcal{S}_{Y}}\left(\gamma^{J}, \xi\right)$ to be the following endomorphism of $\mathcal{S}_{Y}\left(\mathbf{1}_{\mathcal{A} \otimes Y}\right)$

$$
\begin{gathered}
\mathcal{S}_{Y}\left(\mathbf{1}_{\mathcal{A} \otimes Y}\right) \longrightarrow \operatorname{ev}_{y_{1}}\left(\mathcal{S}_{\{*\}}\left(\mathbf{1}_{\mathcal{A}}\right)\right) \stackrel{\xi}{\sim} \operatorname{ev}_{y_{1}}\left(\mathcal{S}_{\{*\}}\left(\operatorname{mult}_{J} \circ \operatorname{mult}_{J}^{R}\left(\mathbf{1}_{\mathcal{A}}\right)\right)\right) \\
\operatorname{ev}_{y_{1}^{J}}\left(\mathcal{S}_{J}\left(\operatorname{mult}_{J}^{R}\left(\mathbf{1}_{\mathcal{A}}\right)\right)\right) \\
\downarrow \operatorname{mon}_{\gamma}^{J} \\
\operatorname{ev}_{y_{2}^{J}}\left(\mathcal{S}_{J}\left(\operatorname{mult}_{J}^{R}\left(\mathbf{1}_{\mathcal{A}}\right)\right)\right) \\
\downarrow \\
\mathcal{S}_{Y}\left(\mathbf{1}_{\mathcal{A} \otimes Y}\right) \longleftarrow \\
\sim \operatorname{ev}_{y_{2}}\left(\mathcal{S}_{\{*\}}\left(\mathbf{1}_{\mathcal{A}}\right)\right) \stackrel{\text { counit }}{\longleftarrow} \operatorname{ev}_{y_{2}}\left(\mathcal{S}_{\{*\}}\left(\operatorname{mult}_{J} \circ \operatorname{mult}_{J}^{R}\left(\mathbf{1}_{\mathcal{A}}\right)\right)\right),
\end{gathered}
$$

where: 
- the first and last isomorphisms are obtained by identifying $\mathbf{1}_{\mathcal{A} \otimes Y}$ with the image of $\mathbf{1}_{\mathcal{A}}$ under

$$
\mathcal{A} \simeq \mathcal{A}^{\{*\}} \rightarrow \mathcal{A}^{Y}
$$

where $\{*\} \rightarrow Y$ is $y_{i}, i=1,2$;

- the third and the fifth isomorphisms are obtained by functoriality with respect to the map $J \rightarrow\{*\}$ in fSet;

2.8.4. In the particular case when $\mathcal{C}=\mathcal{A}^{\otimes Y}$ and $\mathcal{S}_{Y}$ is the identity functor, we will denote the corresponding $\operatorname{Exc}_{\delta_{Y}}\left(\gamma^{J}, \xi\right)$ by

$$
\operatorname{Exc}_{\text {univ }}\left(\gamma^{J}, \xi\right) \in \mathcal{E} n d_{\mathcal{A} \otimes Y}\left(\mathbf{1}_{\mathcal{A} \otimes Y}\right) .
$$

Explicitly, $\operatorname{Exc}_{\text {univ }}\left(\gamma^{J}, \xi\right)$ is given by the composition

$$
\begin{aligned}
\mathbf{1}_{\mathcal{A} \otimes Y} \simeq \iota_{y_{1}}\left(\mathbf{1}_{\mathcal{A}}\right) \stackrel{\xi}{\rightarrow} \iota_{y_{1}} \circ \operatorname{mult}_{J} \circ \operatorname{mult}_{J}^{R}\left(\mathbf{1}_{\mathcal{A}}\right) \simeq \iota_{y_{1}^{J}} \circ \operatorname{mult}_{J}^{R}\left(\mathbf{1}_{\mathcal{A}}\right) \stackrel{\operatorname{mon}_{\gamma^{J}}}{\longrightarrow} \\
\rightarrow \iota_{y_{2}^{J}} \circ \operatorname{mult}_{J}^{R}\left(\mathbf{1}_{\mathcal{A}}\right) \simeq \iota_{y_{2}} \circ \operatorname{mult}_{J} \circ \operatorname{mult}_{J}^{R}\left(\mathbf{1}_{\mathcal{A}} \stackrel{\text { counit }_{\longrightarrow}}{\longrightarrow} \iota_{y_{2}}\left(\mathbf{1}_{\mathcal{A}}\right) \simeq \mathbf{1}_{\mathcal{A} \otimes Y},\right.
\end{aligned}
$$

where $y_{i}$ and $y_{i}^{J}$ denote the functors

$$
\mathcal{A} \rightarrow \mathcal{A}^{Y} \text { and } \mathcal{A}^{J} \rightarrow \mathcal{A}^{Y},
$$

corresponding to

$$
\{*\} \stackrel{y_{i}}{\rightarrow} Y \text { and } J \stackrel{y_{i}^{J}}{\rightarrow} Y,
$$

respectively.

By functoriality, for a general $\mathcal{C}$, the map $\operatorname{Exc}_{\mathcal{S}_{Y}}\left(\gamma^{J}, \xi\right)$, is the image of $\operatorname{Exc}_{\text {univ }}\left(\gamma^{J}, \xi\right)$ under $\mathcal{S}_{Y}$.

2.8.5. Example. Let e be a field of characteristic 0 . Take $\mathcal{A}=\operatorname{Rep}(\mathrm{G})$, and assume that $Y$ has finitely many connected components, so that we have an equivalence

see Theorem 1.5.5.

$$
\operatorname{Rep}(\mathrm{G})^{\otimes Y} \simeq \operatorname{QCoh}\left(\operatorname{LocSys}_{\mathrm{G}}(Y)\right),
$$

Then for $\left(\gamma^{J}, \xi\right)$ as above, we can think of $\operatorname{Exc}_{\text {univ }}\left(\gamma^{J}, \xi\right)$ as an element of

$$
\Gamma\left(\operatorname{LocSys}_{\mathrm{G}}(Y), \mathcal{O}_{\operatorname{LocSys}_{\mathrm{G}}(Y)}\right) \text {. }
$$

Let us describe this element explicitly for a particular (in fact, a generating family) of choices of $\xi$. Let $V_{J}$ be a representation of $G^{J}$. Fix an invariant vector and an invariant covector in $\operatorname{Res}_{G}^{G^{J}}\left(V_{J}\right)$, i.e.,

$$
v: \mathrm{e} \rightarrow \operatorname{Res}_{G}^{G^{J}}\left(V_{J}\right) \text { and } v^{*}: \operatorname{Res}_{G}^{G^{J}}\left(V_{J}\right) \rightarrow \mathrm{e} .
$$

The datum of $v^{*}$ defines by adjunction a map

$$
V_{J} \rightarrow \operatorname{coInd}_{G}^{G^{J}}(\mathrm{e})=\operatorname{mult}_{J}^{R}(\mathrm{e}) .
$$

Let $\xi_{v, v^{*}}$ denote the composite

$$
\mathrm{e} \stackrel{v}{\rightarrow} \operatorname{Res}_{G}^{G^{J}}\left(V_{J}\right)=\operatorname{mult}_{J}\left(V_{J}\right) \stackrel{v^{*}}{\rightarrow} \operatorname{mult}_{J} \circ \operatorname{mult}_{J}^{R}(\mathrm{e}) .
$$

Let us describe explicitly the element

$$
\operatorname{Exc}_{\text {univ }}\left(\gamma^{J}, \xi_{v, v^{*}}\right) \in \Gamma\left(\operatorname{LocSys}_{\mathrm{G}}(Y), \mathcal{O}_{\operatorname{LocSys}_{G}(Y)}\right)
$$

as a function on $\operatorname{LocSys}_{\mathrm{G}}(Y)$.

Namely, the value of this function at a point $\sigma$ of $\operatorname{LocSys}_{\mathrm{G}}(Y)$ is the composite

$$
\begin{gathered}
\mathrm{e} \stackrel{v}{\longrightarrow} \operatorname{ev}_{y_{1}}\left(\left(\operatorname{Res}_{G}^{G^{J}}\left(V_{J}\right)\right)_{\sigma}\right) \stackrel{\sim}{\longrightarrow} \operatorname{ev}_{y_{1}^{J}}\left(\left(V_{J}\right)_{\sigma}\right) \\
\downarrow \\
\mathrm{e} \longleftarrow \mathrm{mon}_{\gamma^{J}} \\
\operatorname{ev}_{y_{2}}\left(\left(\operatorname{Res}_{G}^{G^{J}}\left(V_{J}\right)\right)_{\sigma}\right) \longleftarrow \sim \\
\operatorname{ev}_{y_{2}^{J}}\left(\left(V_{J}\right)_{\sigma}\right),
\end{gathered}
$$


where for $W_{J^{\prime}} \in \operatorname{Rep}\left(G^{J^{\prime}}\right)$ we denote by $\left(W_{J^{\prime}}\right)_{\sigma}$ the corresponding object of $\operatorname{LS}\left(Y^{J^{\prime}}\right)$.

2.8.6. We are now ready to state the main result of this subsection:

Theorem 2.8.7. For $\left(y_{1}, y_{2}, \gamma^{J}\right)$ as above, consider the corresponding map

$$
\gamma^{J}: \Sigma(J) \rightarrow Y
$$

Then the excursion operator

$$
\operatorname{Exc}_{\mathcal{S}_{Y}}\left(\gamma^{J}, \xi\right) \in \operatorname{End}\left(\mathcal{S}_{Y}\left(\mathbf{1}_{\mathcal{A} \otimes Y}\right)\right)
$$

of Sect. 2.8.3 equals the action of the element of $\operatorname{End}_{\mathcal{A} \otimes Y}\left(\mathbf{1}_{\mathcal{A} \otimes Y}\right)$ obtained as the image of $E_{\xi}$ (see (2.14) under the map

$$
\operatorname{End}_{\mathcal{A} \otimes \Sigma(J)}\left(\mathbf{1}_{\mathcal{A} \otimes \Sigma(J)}\right) \stackrel{\gamma^{J}}{\rightarrow} \operatorname{End}_{\mathcal{A} \otimes Y}\left(\mathbf{1}_{\mathcal{A} \otimes Y}\right) .
$$

Remark 2.8.8. We emphasize that the assertion of Theorem 2.8.7 holds for any $\mathcal{A}$ : we do not need either $\mathcal{A}$ to have an affine diagonal. We only use the existence of the base change map

$$
\mathcal{H}_{o m_{\mathcal{A}}}\left(\mathbf{1}_{\mathcal{A}}, \text { mult }_{J} \circ \operatorname{mult}_{J}^{R}\left(\mathbf{1}_{\mathcal{A}}\right)\right) \rightarrow \mathcal{E} n d_{\mathcal{A} \otimes \Sigma(J)}\left(\mathbf{1}_{\mathcal{A} \otimes \Sigma(J)}\right),
$$

but we do not need this map to be an isomorphism.

\subsection{Proof of Theorem 2.8.7.}

2.9.1. First off, since the assertion is functorial in $\mathcal{C}$, it suffices to consider the universal case, namely, $\mathcal{C}=\mathcal{A}^{\otimes Y}$ and $\mathcal{S}_{Y}$ is the identity functor. Second, since the statement is functorial in $Y$, we can assume that

$$
Y=\Sigma(J)
$$

and $\gamma^{J}$ is the tautological $J$-tuple $\gamma_{\text {taut }}^{J}$ of paths

$$
*_{s} \rightarrow *_{t},
$$

where $*_{s}, *_{t}$ are the two points of $\Sigma(J)$.

Thus, we need to show that

$$
\operatorname{Exc}_{\text {univ }}\left(\gamma_{\text {taut }}^{J}, \xi\right)=E_{\xi}
$$

as objects in $\operatorname{End}_{\mathcal{A} \otimes \Sigma(J)}\left(\mathbf{1}_{\mathcal{A} \otimes \Sigma(J)}\right)$.

2.9.2. Let $\mathrm{q}$ denote the map

$$
\mathcal{A}^{\otimes J} \rightarrow \mathcal{A}^{\otimes \Sigma(J)}
$$

corresponding to either circuit in 2.11.

The path $\gamma_{\text {can }}^{J}$ defines a $J$-tuple of isomorphisms of functors

$$
\operatorname{mon}_{\text {can }}^{j}: \iota_{s} \rightarrow \iota_{t}, \quad j \in J
$$

so that the composite

$$
\mathrm{q} \simeq \iota_{s} \circ \text { mult }_{J} \stackrel{\operatorname{mon}_{\text {can }}^{j}}{\longrightarrow} \iota_{t} \circ \text { mult }_{J} \simeq \mathrm{q}
$$

is the identity map for all $j \in J$.

2.9.3. Unwinding the definitions, we obtain that the LHS of 2.17) is the endomorphism of $\mathbf{1}_{\mathcal{A} \otimes \Sigma(J)}$ given by

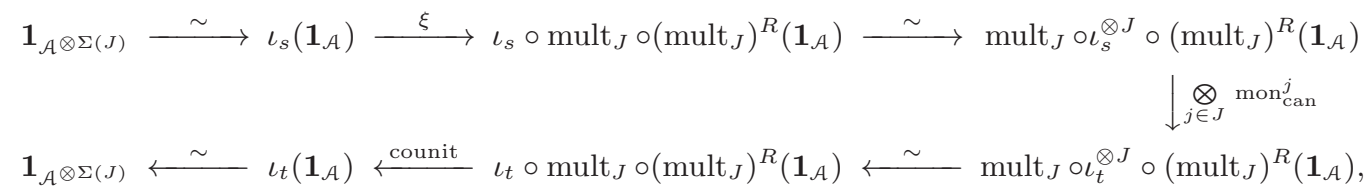

and the RHS is

$$
\mathbf{1}_{\mathcal{A} \otimes \Sigma(J)} \simeq \iota_{s}\left(\mathbf{1}_{\mathcal{A}}\right) \stackrel{\xi}{\rightarrow} \iota_{s} \circ \text { mult }_{J} \circ\left(\text { mult }_{J}\right)^{R}\left(\mathbf{1}_{\mathcal{A}}\right) \stackrel{\mathrm{BC}_{J}}{\longrightarrow} \iota_{s} \circ \iota_{s}^{R} \circ \iota_{t}\left(\mathbf{1}_{\mathcal{A}}\right) \stackrel{\text { counit }}{\longrightarrow} \iota_{t}\left(\mathbf{1}_{\mathcal{A}}\right) \simeq \mathbf{1}_{\mathcal{A} \otimes \Sigma(J)},
$$

where $\mathrm{BC}_{J}$ is an is 2.12). 
2.9.4. We obtain that it suffices to show that the following two natural transformations

$$
\iota_{s} \circ \text { mult }_{J} \circ\left(\text { mult }_{J}\right)^{R} \rightrightarrows \iota_{t}
$$

coincide:

One is the composite

$$
\begin{aligned}
& \iota_{s} \circ \text { mult }_{J} \circ\left(\text { mult }_{J}\right)^{R} \simeq \operatorname{mult}_{J} \circ \iota_{s}^{\otimes J} \circ\left(\text { mult }_{J}\right)^{R} \underset{j \in J}{\otimes} \operatorname{mon}_{\text {can }^{j}} \operatorname{mult}_{J} \circ \iota_{t}^{\otimes J} \circ\left(\text { mult }_{J}\right)^{R} \simeq \\
& \simeq \iota_{t} \circ \text { mult }_{J} \circ\left(\text { mult }_{J}\right)^{R} \rightarrow \iota_{t} .
\end{aligned}
$$

and the other is the composite

$$
\iota_{s} \circ \text { mult }_{J} \circ\left(\text { mult }_{J}\right) \stackrel{R}{\stackrel{\mathrm{BC}_{J}}{\longrightarrow}} \iota_{s} \circ \iota_{s}^{R} \circ \iota_{t} \rightarrow \iota_{t} .
$$

2.9.5. However, unwinding the definition of $\mathrm{BC}_{J}$, we obtain that both (2.18) and (2.19) identify with

$$
\iota_{s} \circ \text { mult }_{J} \circ\left(\text { mult }_{J}\right)^{R} \simeq \mathrm{q} \circ\left(\text { mult }_{J}\right)^{R} \simeq \iota_{t} \circ \text { mult }_{J} \circ\left(\text { mult }_{J}\right)^{R} \rightarrow \iota_{t} .
$$

[Theorem 2.8.7]

\section{TAKING THE TRACE}

In this section we will approach the central theme of this paper: the operation of taking the trace. The usual trace construction assigns to an endomorphism $F$ of a dualizable object $\mathbf{o}$ in a symmetric monoidal category $\mathbf{O}$ its trace $\operatorname{Tr}(F, \mathbf{o})$, which is an endomorphism of the unit object $\mathbf{1}_{\mathbf{O}}$, see Sect. 3.1.1

However, our primary interest will be the notion of higher trace, when $\mathbf{O}$ is actually a symmetric monoidal 2-category. In this case, the trace construction has an additional functoriality, see Sect. 3.2.1 We will apply this formalism in the following two contexts: $\mathbf{O}=$ DGCat, in which our traces will be vector spaces, and $\mathbf{O}=$ Morita(DGCat), in which our traces will be DG-categories.

The main result of this section is Theorem 3.8.5 which describes the interaction between the trace operations at different categorical levels.

\subsection{The usual trace.}

3.1.1. Let $\mathbf{O}$ be a symmetric monoidal category. Given a dualizable object $\mathbf{o} \in \mathbf{O}$ and a point $F \in \operatorname{Endo}(\mathbf{o})$, we define its trace

$$
\operatorname{Tr}(F, \mathbf{o}) \in \operatorname{End}_{\mathbf{O}}\left(\mathbf{1}_{\mathbf{O}}\right)
$$

to be the composite

$$
\mathbf{1}_{\mathbf{O}} \stackrel{\text { unit }}{\longrightarrow} \mathbf{o} \otimes \mathbf{o}^{\vee} \stackrel{F \otimes \mathrm{id}_{\mathrm{O}} \vee}{\longrightarrow} \mathbf{0} \otimes \mathbf{o}^{\vee} \stackrel{\text { counit }}{\longrightarrow} \mathbf{1}_{\mathbf{O}}
$$

3.1.2. The assignment

$$
(\mathbf{o}, F) \mapsto \operatorname{Tr}(F, \mathbf{o})
$$

is symmetric monoidal, i.e., we have a canonical isomorphism

$$
\operatorname{Tr}\left(F_{1} \otimes F_{2}, \mathbf{o}_{1} \otimes \mathbf{o}_{2}\right) \simeq \operatorname{Tr}\left(F_{1}, \mathbf{o}_{1}\right) \cdot \operatorname{Tr}\left(F_{2}, \mathbf{o}_{2}\right),
$$

where $\cdot$ denotes the structure of commutative monoid on $\operatorname{End}\left(\mathbf{1}_{\mathbf{O}}\right)$ induced by the symmetric monoidal structure on $\mathbf{O}$, see [TV, Sect. 2.5], along with higher compatibilities.

In particular

$$
\operatorname{Tr}\left(\operatorname{id}_{\mathbf{1}_{\mathbf{O}}}, \mathbf{1}_{\mathbf{O}}\right)=\operatorname{id}_{\mathbf{1}_{\mathbf{O}}}=\mathbf{1}_{\operatorname{End}_{\mathbf{O}}(\mathbf{o})}
$$


3.1.3. For a morphism $F: \mathbf{o}_{1} \rightarrow \mathbf{o}_{2}$ between dualizable objects let $F^{\vee}$ denote the dual morphism $\mathbf{o}_{2}^{\vee} \rightarrow \mathbf{o}_{1}^{\vee}$.

Let $q_{F}$ be the point in $\operatorname{Maps}\left(\mathbf{1}_{\mathbf{O}}, \mathbf{o}_{2} \otimes \mathbf{o}_{1}^{\vee}\right)$ that represents $F$, i.e., the composite

$$
\mathbf{1}_{\mathbf{O}} \stackrel{\text { unit }}{\longrightarrow} \mathbf{o}_{1} \otimes \mathbf{o}_{1}^{\vee} \stackrel{F \otimes \mathrm{id}_{\mathbf{o}}^{\vee}}{\longrightarrow} \mathbf{o}_{2} \otimes \mathbf{o}_{1}^{\vee}
$$

We have

$$
q_{F}=q_{F^{\vee}} .
$$

From here it follows that for every $\mathbf{o}$ and $F$ as in Sect. 3.1.1 we have

$$
\operatorname{Tr}(F, \mathbf{o})=\operatorname{Tr}\left(F^{\vee}, \mathbf{o}^{\vee}\right) .
$$

3.1.4. Similarly, the trace map has the following cyclicity property: we claim that for morphisms

$$
F_{1,2}: \mathbf{o}_{1} \rightarrow \mathbf{o}_{2} \text { and } F_{2,1}: \mathbf{o}_{2} \rightarrow \mathbf{o}_{1},
$$

there is a canonical isomorphism

$$
\operatorname{Tr}\left(F_{1,2} \circ F_{2,1}, \mathbf{o}_{2}\right) \simeq \operatorname{Tr}\left(F_{2,1} \circ F_{1,2}, \mathbf{o}_{1}\right) .
$$

Indeed, let $q_{i, j} \in \operatorname{Maps}\left(\mathbf{1}_{\mathbf{O}}, \mathbf{o}_{j} \otimes \mathbf{o}_{i}^{\vee}\right)$ be the point that represents $F_{i, j}$. Then $F_{i, j} \circ F_{j, i}$ is represented by the map

$$
\mathbf{1}_{\mathbf{O}} \simeq \mathbf{1}_{\mathbf{O}} \otimes \mathbf{1}_{\mathbf{O}} \stackrel{q_{i, j} \otimes q_{j, i}}{\longrightarrow} \mathbf{o}_{j} \otimes \mathbf{o}_{i}^{\vee} \otimes \mathbf{o}_{i} \otimes \mathbf{o}_{j}^{\vee} \stackrel{\mathrm{id}_{\mathbf{o}_{j}} \otimes{\text { counit } \otimes \mathrm{id}_{\mathbf{o}_{j}^{\vee}}}_{\longrightarrow}^{\longrightarrow}}{\longrightarrow} \mathbf{o}_{j} \otimes \mathbf{1}_{\mathbf{O}} \otimes \mathbf{o}_{j}^{\vee} \simeq \mathbf{o}_{j} \otimes \mathbf{o}_{j}^{\vee} .
$$

Hence,

is the composite

$$
\operatorname{Tr}\left(F_{i, j} \circ F_{j, i}, \mathbf{o}_{j}\right)
$$

$$
\mathbf{1}_{\mathbf{O}} \simeq \mathbf{1}_{\mathbf{O}} \otimes \mathbf{1}_{\mathbf{O}} \stackrel{q_{i, j} \otimes q_{j, i}}{\longrightarrow} \mathbf{o}_{j} \otimes \mathbf{o}_{i}^{\vee} \otimes \mathbf{o}_{i} \otimes \mathbf{o}_{j}^{\vee} \stackrel{\mathrm{id}_{\mathbf{o}_{j}} \otimes \operatorname{counit} \otimes \mathrm{id}_{\mathbf{o}_{j}^{\vee}}}{\longrightarrow} \mathbf{o}_{j} \otimes \mathbf{1}_{\mathbf{O}} \otimes \mathbf{o}_{j}^{\vee} \simeq \mathbf{o}_{j} \otimes \mathbf{o}_{j}^{\vee} \stackrel{\text { counit }}{\longrightarrow} \mathbf{1}_{\mathbf{O}},
$$

and the latter expression is manifestly symmetric in $i$ and $j$.

\subsection{Trace in a 2-category.}

3.2.1. Let now $\mathbf{O}$ be a symmetric monoidal 2-category (we will be assuming the formalism of $(\infty, 2)$ categories from [GR1, Chapter 10]).

Let $\mathbf{o}_{1}$ and $\mathbf{o}_{2}$ be a pair of dualizable objects, each endowed with an endomorphism $F_{i}$. Let $t$ : $\mathbf{o}_{1} \rightarrow \mathbf{o}_{2}$ be a 1-morphism that admits a right adjoint. This means that there exists a 1-morphism $t^{R}: \mathbf{o}_{2} \rightarrow \mathbf{o}_{1}$ and 2-morphisms

$$
\operatorname{id}_{\mathbf{o}_{1}} \rightarrow t^{R} \circ t \text { and } t \circ t^{R} \rightarrow \mathrm{id}_{\mathbf{o}_{2}}
$$

that satisfy the usual axioms, see GR1, Chapter 12, Sect. 1].

Let $F_{i}$ be a 1-endomorphism of $\mathbf{o}_{i}$. In addition, let us be given a 2-morphism

$$
\alpha: t \circ F_{1} \rightarrow F_{2} \circ t
$$

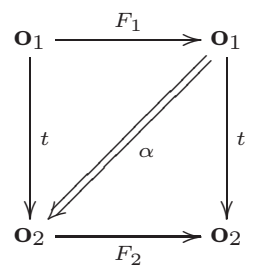

In this case, following [BN1 Definition 2.24] or [KP1, Example 1.2.5], we define the 2-morphism

$$
\operatorname{Tr}(t, \alpha): \operatorname{Tr}\left(F_{1}, \mathbf{o}_{1}\right) \rightarrow \operatorname{Tr}\left(F_{2}, \mathbf{o}_{2}\right)
$$


to be the composite

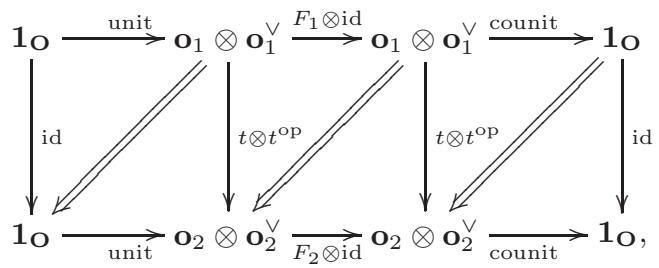

where:

$-t^{\mathrm{op}}$ denotes the 1-morphism $\mathbf{o}_{1}^{\vee} \rightarrow \mathbf{o}_{2}^{\vee}$ equal to $\left(t^{R}\right)^{\vee}$;

-the 2-morphism in the left square is given by the $\left(t, t^{R}\right)$-adjunction;

-the 2-morphism in the middle square is given by $\alpha$;

-the 2-morphism in the right square is given by the $\left(t, t^{R}\right)$-adjunction.

Remark 3.2.2. The above construction is equivalent to formula 0.3 given earlier.

3.2.3. The construction of Sect. 3.2.1 is compatible with compositions:

For a composition

$$
\mathbf{o}_{1} \stackrel{t_{1,2}}{\rightarrow} \mathbf{o}_{2} \stackrel{t_{2,3}}{\rightarrow} \mathbf{o}_{3}
$$

the 2-morphism

$$
\operatorname{Tr}\left(F_{1}, \mathbf{o}_{1}\right) \stackrel{\operatorname{Tr}\left(t_{1,2}, \alpha_{1,2}\right)}{\longrightarrow} \operatorname{Tr}\left(F_{2}, \mathbf{o}_{2}\right) \stackrel{\operatorname{Tr}\left(t_{2,3}, \alpha_{2,3}\right)}{\longrightarrow} \operatorname{Tr}\left(F_{3}, \mathbf{o}_{3}\right)
$$

identifies with $\operatorname{Tr}\left(t_{1,3}, \alpha_{1,3}\right)$, where

$$
t_{1,3}=t_{2,3} \circ t_{1,2},
$$

and $\alpha_{1,3}$ is obtained by composing $\alpha_{1,2}$ and $\alpha_{2,3}$.

3.2.4. The construction of Sect. 3.2.1 has the following cyclicity property:

Given a pair of diagrams of 2-morphisms
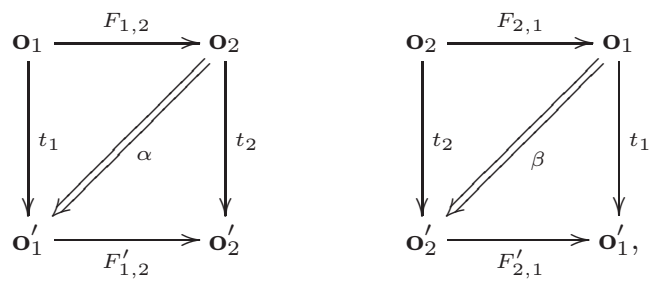

we can compose them horizontally in two ways, thus getting diagrams
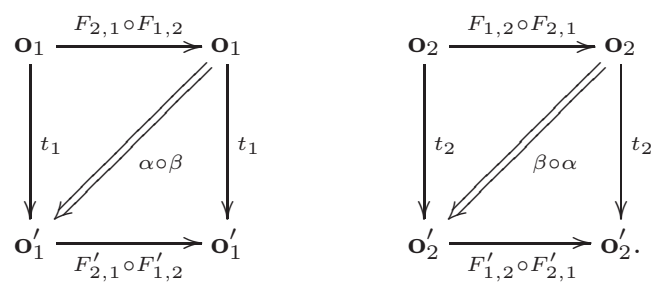

Assume now that objects $\mathbf{o}_{1}, \mathbf{o}_{2}, \mathbf{o}_{1}^{\prime}$ and $\mathbf{o}_{2}^{\prime}$ are dualizable, while 1-morphisms $t_{1}$ and $t_{2}$ admit right adjoints. Then we claim that the trace maps

$$
\operatorname{Tr}\left(t_{1}, \alpha \circ \beta\right): \operatorname{Tr}\left(F_{2,1} \circ F_{1,2}, \mathbf{o}_{1}\right) \rightarrow \operatorname{Tr}\left(F_{2,1}^{\prime} \circ F_{1,2}^{\prime}, \mathbf{o}_{1}^{\prime}\right)
$$

and

$$
\operatorname{Tr}\left(t_{2}, \beta \circ \alpha\right): \operatorname{Tr}\left(F_{1,2} \circ F_{2,1}, \mathbf{o}_{2}\right) \rightarrow \operatorname{Tr}\left(F_{1,2}^{\prime} \circ F_{2,1}^{\prime}, \mathbf{o}_{2}^{\prime}\right)
$$


match up under identifications

$$
\operatorname{Tr}\left(F_{1,2} \circ F_{2,1}, \mathbf{o}_{2}\right) \simeq \operatorname{Tr}\left(F_{2,1} \circ F_{1,2}, \mathbf{o}_{1}\right) \text { and } \operatorname{Tr}\left(F_{1,2}^{\prime} \circ F_{2,1}^{\prime}, \mathbf{o}_{2}^{\prime}\right) \simeq \operatorname{Tr}\left(F_{2,1}^{\prime} \circ F_{1,2}^{\prime}, \mathbf{o}_{1}^{\prime}\right)
$$

of 3.2 .

More precisely, we claim that there is a canonical isomorphism

$$
\operatorname{Tr}\left(t_{1}, \alpha \circ \beta\right) \simeq \operatorname{Tr}\left(t_{2}, \beta \circ \alpha\right)
$$

in

$$
\text { Funct }\left(\operatorname{Tr}\left(F_{2,1} \circ F_{1,2}, \mathbf{o}_{1}\right), \operatorname{Tr}\left(F_{2,1}^{\prime} \circ F_{1,2}^{\prime}, \mathbf{o}_{1}^{\prime}\right)\right) \simeq \operatorname{Funct}\left(\operatorname{Tr}\left(F_{1,2} \circ F_{2,1}, \mathbf{o}_{2}\right), \operatorname{Tr}\left(F_{1,2}^{\prime} \circ F_{2,1}^{\prime}, \mathbf{o}_{2}^{\prime}\right)\right) \text {. }
$$

Indeed, let $q_{i, j} \in \operatorname{Maps}\left(\mathbf{1}_{\mathbf{O}}, \mathbf{o}_{j} \otimes \mathbf{o}_{i}^{\vee}\right)$ and $q_{i, j}^{\prime} \in \operatorname{Maps}\left(\mathbf{1}_{\mathbf{O}}, \mathbf{o}_{j}^{\prime} \otimes \mathbf{o}_{i}^{\prime \vee}\right)$ be the points that represent $F_{i, j}$ and $F_{i, j}^{\prime}$, respectively. Then arguing as in Sect. 3.1.4 one sees that both maps

$$
\operatorname{Tr}\left(t_{1}, \alpha \circ \beta\right) \text { and } \operatorname{Tr}\left(t_{2}, \beta \circ \alpha\right)
$$

are canonically isomorphic to the composite

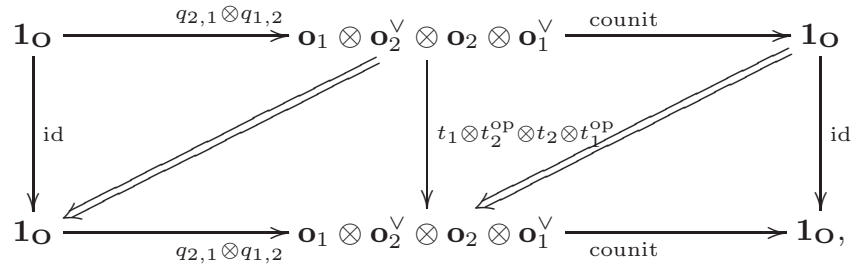

where:

$-t_{i}^{\text {op }}$ denotes the 1-morphism $\mathbf{o}_{i}^{\vee} \rightarrow \mathbf{o}_{i}^{\prime \vee}$ equal to $\left(t_{i}^{R}\right)^{\vee}$

-the 2-morphism in the left square is given by the $\left(t_{i}, t_{j}^{R}\right)$-adjunctions and 2-morphisms $\alpha$ and $\beta$;

-the 2-morphism in the right square is given by the $\left(t_{i}, t_{i}^{R}\right)$-adjunction.

3.2.5. By functoriality, isomorphisms (3.5) from Sect. 3.2.4 are compatible with vertical compositions:

Consider a pair of diagrams of 2-morphisms
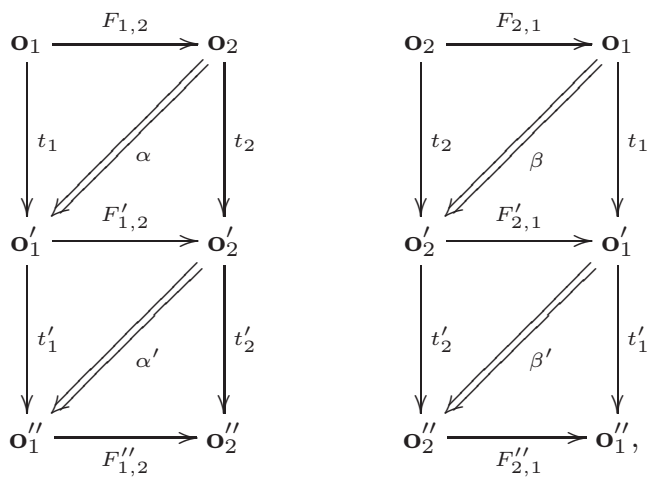

in which all objects are dualizable and all vertical 1-morphisms admit right adjoints.

Then isomorphisms (3.5), corresponding to the top part, the bottom part and the vertical composition of the diagrams (3.6), respectively, are

$$
\operatorname{Tr}\left(t_{1}, \alpha \circ \beta\right) \simeq \operatorname{Tr}\left(t_{2}, \beta \circ \alpha\right), \quad \operatorname{Tr}\left(t_{1}^{\prime}, \alpha^{\prime} \circ \beta^{\prime}\right) \simeq \operatorname{Tr}\left(t_{2}^{\prime}, \beta^{\prime} \circ \alpha^{\prime}\right)
$$

$$
\operatorname{Tr}\left(t_{1}^{\prime} \circ t_{1},\left(\alpha^{\prime} \circ \alpha\right) \circ\left(\beta^{\prime} \circ \beta\right)\right) \simeq \operatorname{Tr}\left(t_{2}^{\prime} \circ t_{2},\left(\beta^{\prime} \circ \beta\right) \circ\left(\alpha^{\prime} \circ \alpha\right)\right)
$$


Moreover, by the fuctoriality of (3.5) the following diagram is commutative:

$$
\begin{array}{cc}
\operatorname{Tr}\left(t_{1}^{\prime} \circ t_{1},\left(\alpha^{\prime} \circ \alpha\right) \circ\left(\beta^{\prime} \circ \beta\right)\right) \stackrel{\text { Sect. } 3.2 .3}{\sim} \operatorname{Tr}\left(t_{1}^{\prime}, \alpha^{\prime} \circ \beta^{\prime}\right) \circ \operatorname{Tr}\left(t_{1}, \alpha \circ \beta\right) \\
\downarrow \\
\operatorname{Tr.8} \mid \sim \\
\operatorname{Tr}\left(t_{2}^{\prime} \circ t_{2},\left(\beta^{\prime} \circ \beta\right) \circ\left(\alpha^{\prime} \circ \alpha\right)\right) \frac{\text { Sect. B.2.3 }}{\sim} \operatorname{Tr}\left(t_{2}^{\prime}, \beta^{\prime} \circ \alpha^{\prime}\right) \circ \operatorname{Tr}\left(t_{2}, \beta \circ \alpha\right) .
\end{array}
$$

3.3. Properties of the 2-categorical trace. In this subsection we will explore further functoriality properties of the construction of Sect. 3.2.1. We recommend the reader to skip this subsection and return to it when necessary.

3.3.1. The functoriality mentioned in Sect. 3.2.3 can be promoted to a symmetric monoidal functor between symmetric monoidal $(\infty, 1)$-categories:

Consider the category, to be denoted $L(\mathbf{O})$, whose objects are pairs $(\mathbf{o}, F)$, where $\mathbf{o} \in \mathbf{O}$ and $F \in \operatorname{End}_{\mathbf{O}}(\mathbf{o})$, and whose morphisms are given by diagrams (3.3), see [KP1, Sect. 1.2 10 .

The symmetric monoidial structure on $\mathbf{O}$ induces one on $L(\mathbf{O})$. Let $L(\mathbf{O})_{\mathrm{rgd}} \subset L(\mathbf{O})$ be the 1-full subcategory, where we allow as objects those $(\mathbf{o}, F)$ for which $\mathbf{o}$ is dualizable as an object of $\mathbf{O}$, and where we restrict 1-morphisms to those pairs $(t, \alpha)$, for which $t$ admits a right adjoint.

Then the assignment

$$
(\mathbf{o}, F) \mapsto \operatorname{Tr}(F, \mathbf{o})
$$

is a symmetric monoidal functor

$$
\operatorname{Tr}: L(\mathbf{O})_{\mathrm{rgd}} \rightarrow \operatorname{Endo}\left(\mathbf{1}_{\mathbf{O}}\right) .
$$

The construction of the functor (3.10) can be either performed directly using the definition of $\infty$ categorical symmetric monoidal structures as in GR1, Chapter 1, Sect. 3.3], or using the device of HSS, Theorem 1.7].

3.3.2. Let $\mathbf{a} \in \mathbf{O}$ be an associative/commutative algebra object in $\mathbf{O}$, so that the multiplication map

$$
\mathbf{a} \otimes \mathbf{a} \rightarrow \mathbf{a}
$$

admits a right adjoint. Assume also that $\mathbf{a}$ is dualizable as an object of $\mathbf{O}$.

Let $F_{\mathbf{a}}$ be a right-lax monoidal/symmetric monoidal endomorphism of a (see, e.g., Sect. 3.4.4). Then $\left(\mathbf{a}, F_{\mathbf{a}}\right)$ acquires a structure of associative/commutative algebra object in $L(\mathbf{O})_{\text {rgd }}$.

Hence, we obtain that $\operatorname{Tr}\left(F_{\mathbf{a}}, \mathbf{a}\right)$ acquires a structure of associative/commutative algebra object in $\operatorname{Endo}\left(\mathbf{1}_{0}\right)$.

3.3.3. Let $\mathbf{a}$ be as above, and let $\mathbf{m} \in \mathbf{O}$ be an $\mathbf{a}$-module object. Assume that the action map

$$
\mathbf{a} \otimes \mathbf{m} \rightarrow \mathbf{a}
$$

admits a right adjoint. Assume also that $\mathbf{m}$ is dualizable as an object of $\mathbf{O}$.

Let $F_{\mathbf{m}}$ be an endomorphism of $\mathbf{m}$ that is right-lax compatible with $F_{\mathbf{a}}$ (see, e.g., Sect. 3.4.4). Then $\left(\mathbf{m}, F_{\mathbf{m}}\right)$ acquires a structure of module over $\left(\mathbf{a}, F_{\mathbf{a}}\right)$ in $L(\mathbf{O})_{\mathrm{rgd}}$.

Hence, we obtain that $\operatorname{Tr}\left(F_{\mathbf{m}}, \mathbf{m}\right) \in \operatorname{End}_{\mathbf{O}}\left(\mathbf{1}_{\mathbf{O}}\right)$ acquires a structure of module over $\operatorname{Tr}\left(F_{\mathbf{a}}, \mathbf{a}\right)$.

\footnotetext{
${ }^{10}$ To see the full construction of $L(\mathbf{O})$ as an $\infty$-category see GR1 Chapter 10, Sect. 4.1].
} 
3.3.4. Let $\mathbf{a}$ and $\mathbf{a}^{\prime}$ be a pair of associative/commutative algebra objects in $\mathbf{O}$ as in Sect. 3.3 .2 each endowed with a right-lax monoidal/symmetric monoidal endomorphism. Let $\varphi$ be a right-lax monoidal/symmetric monoidal map $\mathbf{a} \rightarrow \mathbf{a}^{\prime}$, equipped with a 2-morphism in the diagram

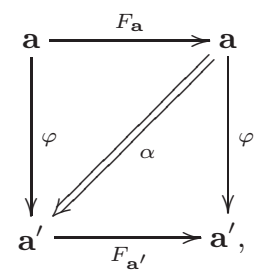

compatible with the right-lax monoidal/symmetric monoidal structures on the edges. Assume that $\varphi$ admits a right adjoint.

We can view the data of $(\varphi, \alpha)$ as a morphism of associative/commutative algebra objects $\left(\mathbf{a}, F_{\mathbf{a}}\right) \rightarrow$ $\left(\mathbf{a}^{\prime}, F_{\mathbf{a}^{\prime}}\right)$ in $L(\mathbf{O})_{\mathrm{rgd}}$. Hence, the above data induces a map

$$
\operatorname{Tr}\left(F_{\mathbf{a}}, \mathbf{a}\right) \rightarrow \operatorname{Tr}\left(F_{\mathbf{a}^{\prime}}, \mathbf{a}^{\prime}\right)
$$

which is a map of associative/commutative algebras, as follows from the functoriality of the trace construction, see Sect. 3.2 .3

3.3.5. Note that $\left(\mathbf{1}_{\mathbf{O}}, \mathrm{id}\right)$ is the unit in $L(\mathbf{O})_{\mathrm{rgd}}$ (see Sect. 3.3.1). Since $\mathrm{Tr}$ is a symmetric monoidal functor, we can identify

as objects of $\operatorname{Endo}\left(\mathbf{1}_{\mathbf{O}}\right)$.

$$
\operatorname{Tr}\left(\mathrm{id}, \mathbf{1}_{\mathbf{O}}\right)=\mathbf{1}_{\mathrm{End}_{\mathbf{O}}\left(\mathbf{1}_{\mathbf{O}}\right)}
$$

Let now $\mathbf{a}$ be as in Sect. 3.3.2 Consider the unit map

$$
1_{\mathrm{O}} \rightarrow \mathbf{a}
$$

equipped with the 2-morphism

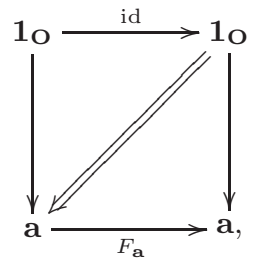

provided by the right-lax monoidal structure on $F_{\mathbf{a}}$. Assume that (3.11) admits a right adjoint.

It follows from Sect. 3.3.4 that the resulting map

$$
\mathbf{1}_{\mathrm{End}_{\mathbf{O}}\left(\mathbf{1}_{\mathbf{O}}\right)} \simeq \operatorname{Tr}\left(\mathrm{id}, \mathbf{1}_{\mathbf{O}}\right) \rightarrow \operatorname{Tr}\left(F_{\mathbf{a}}, \mathbf{a}\right)
$$

is the unit of $\operatorname{Tr}\left(F_{\mathbf{a}}, \mathbf{a}\right)$ as an associative/commutative algebra object in Endo(10).

\subsection{Trace on DG categories.}

3.4.1. The example of primary interest for us is $\mathbf{O}=$ DGCat, with its natural symmetric monoidal structure.

Note that $\mathbf{1}_{\mathrm{DGCat}}=$ Vect, so

$$
\operatorname{End}\left(\mathbf{1}_{\text {DGCat }}\right) \simeq \text { Vect }
$$

as a category, equipped with a symmetric monoidal structure.

Hence, for a dualizable DG category $\mathcal{C}$ equipped with an endofunctor $F$, we obtain an object

$$
\operatorname{Tr}(F, \mathcal{C}) \in \text { Vect } .
$$


Furthermore, if $T: \mathcal{C}_{1} \rightarrow \mathcal{C}_{2}$ is a morphism in DGCat (i.e., a colimit-preserving e-linear exact functor) between dualizable DG categories that admits a continuous right adjoint, and given a natural transformation

$$
\alpha: T \circ F_{1} \rightarrow F_{2} \circ T
$$

we obtain a map in Vect

$$
\operatorname{Tr}(T, \alpha): \operatorname{Tr}\left(F_{1}, \mathrm{C}_{1}\right) \rightarrow \operatorname{Tr}\left(F_{2}, \mathrm{C}_{2}\right)
$$

3.4.2. Take $\mathrm{C}=$ Vect and $F=\mathrm{Id}$. Then by (3.1), we have

$$
\operatorname{Tr}(\mathrm{Id}, \mathrm{Vect}) \simeq \mathrm{e},
$$

as an object in (3.13).

More generally, for $V \in$ Vect, the trace of the endofunctor of Vect, given by $-\otimes V$, identifies with $V$ as an object in (3.13).

3.4.3. Take $\mathcal{C}_{2}=\mathcal{C}$ and $\mathcal{C}_{1}=$ Vect with the functor $T$ corresponding to an object $c \in \mathcal{C}$, i.e., the (unique) colimit preserving functor satisfying

$$
\mathrm{e} \mapsto c
$$

Note that the condition that $T$ admit a continuous right adjoint is equivalent to the condition that $c$ be compact.

Let $F_{2}=F$ be some endofunctor of $\mathcal{C}$ and take $F_{1}=\mathrm{Id}$. Then the datum of $\alpha$ amounts to a morphism

$$
\alpha: c \rightarrow F(c)
$$

The resulting map

$$
\mathrm{e} \simeq \operatorname{Tr}(\mathrm{Id}, \mathrm{Vect}) \stackrel{\operatorname{Tr}(T, \alpha)}{\longrightarrow} \operatorname{Tr}(F, \mathrm{C})
$$

corresponds to a point in $\operatorname{Tr}(F, \mathcal{C})$, which we will denote by $\operatorname{cl}(c, \alpha)$.

3.4.4. Let $\mathcal{R}$ be a monoidal/symmetric monoidal DG category, and $\mathcal{M}$ an $\mathcal{R}$-module category. Assume that the functors

$$
\mathcal{R} \otimes \mathcal{R} \rightarrow \mathcal{R} \text { and } \mathcal{R} \otimes \mathcal{M} \rightarrow \mathcal{M}
$$

viewed as functors of plain DG categories admit right adjoints (if $\mathcal{R}$ and $\mathcal{M}$ are compactly generated, the condition of admitting a right adjoint is equivalent to preserving compactness).

Let $\mathcal{R}$ be endowed with a right-lax monoidal/symmetric monoidal endofunctor $F_{\mathcal{R}}$, i.e., we have a natural transformation

$$
F_{\mathcal{R}}\left(a_{1}\right) \otimes F_{\mathcal{R}}\left(a_{2}\right) \rightarrow F_{\mathcal{R}}\left(a_{1} \otimes a_{2}\right), \quad a_{1}, a_{2} \in \mathcal{R}
$$

equipped with higher compatibilities.

Assume also that $\mathcal{M}$ is dualizable and is endowed with an endofunctor $\phi_{\mathcal{M}}$ that is right-lax compatible with the $\mathcal{R}$-action. I.e., we have a natural transformation

$$
F_{\mathcal{R}}(a) \otimes F_{\mathcal{M}}(m) \rightarrow F_{\mathcal{X}}(a \otimes m), \quad a \in \mathcal{R}, m \in \mathcal{M},
$$

equipped with higher compatibilities.

In this case, applying the construction of Sects. 3.3 .2 and 3.3 .3 , we obtain that $\operatorname{Tr}\left(F_{\mathcal{R}}, \mathcal{R}\right)$ acquires a structure of associative/commutative algebra, and $\operatorname{Tr}\left(F_{\mathcal{M}}, \mathcal{M}\right)$ acquires a structure of $\operatorname{Tr}\left(F_{\mathcal{R}}, \mathcal{R}\right)$-module. 
3.4.5. Let $\mathcal{R}$ and $\mathcal{M}$ be as above. Let $r \in \mathcal{R}$ be a compact object equipped with a map $\alpha: r \rightarrow F_{\mathcal{R}}(r)$. On the one hand, we can consider

$$
\operatorname{cl}(r, \alpha) \in \operatorname{Tr}\left(F_{\mathcal{R}}, \mathcal{R}\right) .
$$

On the other hand, let $H_{r}$ denote the endofunctor of $\mathcal{M}$ given by the action of $r$ (here " $H$ " should be evocative of "Hecke"). The right-lax compatibility of $F_{\mathcal{M}}$ with the action defines a natural transformation

$$
H_{r} \circ F_{\mathcal{M}} \stackrel{\alpha}{\rightarrow} H_{F_{\mathcal{R}}(r)} \circ F_{\mathcal{M}} \rightarrow F_{\mathcal{M}} \circ H_{r}
$$

which we denote by $\alpha_{r, \mathcal{M}}$.

By Sect. 3.2.1 to the pair $\left(H_{r}, \alpha_{r, \mathcal{M}}\right)$ we can assign the map

$$
\operatorname{Tr}\left(H_{r}, \alpha_{r, \mathcal{M}}\right): \operatorname{Tr}\left(F_{\mathcal{M}}, \mathcal{M}\right) \rightarrow \operatorname{Tr}\left(F_{\mathcal{M}}, \mathcal{M}\right) .
$$

We claim:

Proposition 3.4.6. The action of $\operatorname{cl}(r, \alpha)$ on $\operatorname{Tr}\left(F_{\mathcal{M}}, \mathcal{M}\right)$ equals $\operatorname{Tr}\left(H_{r}, \alpha_{r, \mathcal{M}}\right)$.

Proof. Follows from the functoriality of the trace construction (see Sect. 3.2.3) corresponding to the composition

$$
\mathcal{M} \simeq \operatorname{Vect} \otimes \mathcal{M} \stackrel{r \otimes I d}{\longrightarrow} \mathcal{R} \otimes \mathcal{M} \stackrel{\text { act }}{\longrightarrow} \mathcal{M} .
$$

3.5. Examples. The results from this subsection will not be used in the rest of the paper. However, they are meant to provide an intuition for the behavior of the categorical trace construction.

3.5.1. Consider the example $\mathrm{C}=R$-mod, where $R \in \operatorname{AssocAlg}$ (Vect). We have

$$
R_{1}-\bmod \otimes R_{2}-\bmod \simeq\left(R_{1} \otimes R_{2}\right)-\bmod ,
$$

see [Lu2, Theorem 4.8.5.16].

In particular, $(R \text {-mod })^{\vee}$ identifies with $R^{\text {rev }}$-mod, where $R^{\text {rev }}$ is obtained from $R$ by reversing the multiplication. The unit and counit map are given by the functors

$$
\text { Vect } \rightarrow\left(R \otimes R^{\mathrm{rev}}\right) \text {-mod, } \mathrm{e} \mapsto R,
$$

and

$$
\left(R \otimes R^{\mathrm{rev}}\right) \text {-mod } \rightarrow \text { Vect, } \quad Q \mapsto R \underset{R \otimes R^{\mathrm{rev}}}{\otimes} Q
$$

respectively.

Identifying

$$
\operatorname{End}(R-\bmod ) \simeq R-\bmod \otimes(R-\bmod )^{\vee} \simeq\left(R \otimes R^{\mathrm{rev}}\right)-\bmod ,
$$

we obtain that every continuous endofunctor of $R$-mod is of the form

$$
M \mapsto F_{Q}(M):=\underset{R}{\otimes} \underset{R}{\otimes} M
$$

for $Q \in\left(R \otimes R^{\text {rev }}\right)$-mod.

The trace of such an endofunctor is given by

$$
\mathrm{HH}_{\bullet}(R, Q):=R \underset{R \otimes R^{\mathrm{rev}}}{\otimes} Q .
$$

The identity endofunctor of $R$-mod corresponds to $Q=R$. In this case we use a simplified notation

$$
\mathrm{HH}_{\bullet}(R):=\mathrm{HH}_{\bullet}(R, Q) \text {. }
$$

This is the vector space of Hochschild chains on $R$. 
3.5.2. Let $y$ be a prestack (see GR1, Chapter 2, Sect. 1]), such that:

-the category $\mathrm{QCoh}(\mathrm{y})$ is dualizable;

-the object $\mathcal{O}_{y} \in \mathrm{QCoh}(\mathrm{y})$ is compact;

-the diagonal morphism $\Delta: y \rightarrow y \times y$ is schematic and qsqc (quasi-separated and quasi-compact).

For example, these conditions are satisfied for a quasi-compact algebraic stack with affine diagonal, of finite type over a field of characteristic 0 (see [DrGa1, Theorem 1.4.2]).

The condition that $\mathcal{O}_{y} \in \mathrm{QCoh}(y)$ is compact is equivalent to one saying that the functor of global sections

$$
\Gamma(y,-): \mathrm{QCoh}(y) \rightarrow \text { Vect }
$$

is continuous. The condition that $\Delta$ is schematic and qsqc implies that the direct image functor

$$
\Delta_{*}: \mathrm{QCoh}(y) \rightarrow \mathrm{QCoh}(y \times y)
$$

is continuous and satisfies base change.

In this case, the functors

$$
\operatorname{Vect} \stackrel{\mathrm{e} \mapsto \mathrm{O}_{y}}{\rightarrow} \mathrm{QCoh}(y) \stackrel{\Delta_{*}}{\rightarrow} \mathrm{QCoh}(y \times y) \simeq \mathrm{QCoh}(y) \otimes \mathrm{QCoh}(y)
$$

and

$$
\mathrm{QCoh}(y) \otimes \mathrm{QCoh}(y) \simeq \mathrm{QCoh}(y \times y) \stackrel{\Delta^{*}}{\rightarrow} \mathrm{QCoh}(y) \stackrel{\Gamma\left(y_{-}-\right)}{\rightarrow} \mathrm{Vect}
$$

define an identification

$$
\mathrm{QCoh}(y)^{\vee} \simeq \mathrm{QCoh}(y)
$$

(the proof is a diagram chase using base change).

3.5.3. Let $\phi$ be an endomorphism of $y$ and consider the endofunctor of QCoh( $y)$ given by $\phi^{*}$.

We claim that $\operatorname{Tr}\left(\phi^{*}, \mathrm{QCoh}(y)\right)$ identifies canonically with

$$
\Gamma\left(y^{\phi}, \mathcal{O}_{y \phi}\right)
$$

where

$$
y^{\phi}:=y \underset{\operatorname{Graph}_{\phi}, y \times y, \Delta}{x} y
$$

is the fixed point locus of $\phi$.

Indeed, let $\iota$ denote the forgetful map $y^{\phi} \rightarrow y$, so that we have

$$
\iota \circ \phi \simeq \iota \text {. }
$$

We calculate $\operatorname{Tr}\left(\phi^{*}, \mathrm{QCoh}(y)\right)$ as the composite

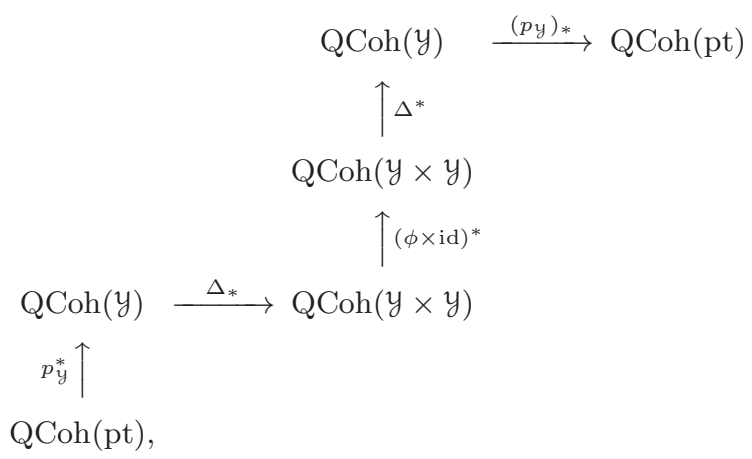

where $p_{y}: y \rightarrow$ pt is the projection map. 
By base change, we rewrite this functor as

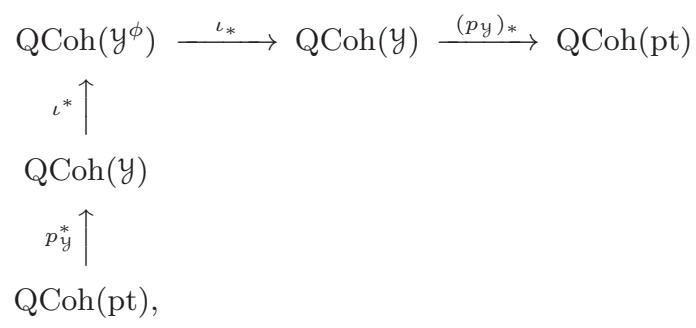

which sends e $\in$ Vect $=$ QCoh(pt) to $\Gamma\left(y^{\phi}, \mathcal{O}_{y \phi}\right)$, as desired.

3.5.4. Let now $\mathcal{F} \in \mathrm{QCoh}(y)$ be a compact object, equipped with a map

$$
\alpha: \mathcal{F} \rightarrow \phi^{*}(\mathcal{F}) \text {. }
$$

Consider the corresponding object

$$
\operatorname{cl}(\mathcal{F}, \alpha) \in \operatorname{Tr}\left(\phi^{*}, \mathrm{Q} \operatorname{Coh}(y)\right),
$$

see Sect. 3.4.3.

We will now describe explicitly the image of $\operatorname{cl}(\mathcal{F}, \alpha)$ under the identification

$$
\operatorname{Tr}\left(\phi^{*}, \mathrm{Q} \operatorname{Coh}(y)\right) \simeq \Gamma\left(y^{\phi}, \mathcal{O}_{y_{\phi}}\right) .
$$

First, we claim:

Lemma 3.5.5. Every compact object $\mathcal{F} \in \mathrm{QCoh}(\mathrm{y})$ is dualizable in the sense of the symmetric monoidal structure on $\mathrm{QCoh}(\mathrm{y})$.

Proof. The proof runs parallel to [BFN, Proposition 3.6]:

Since the diagonal morphism of $y$ is schematic and qsqc, any map

$$
f: S \rightarrow y,
$$

where $S$ an affine scheme, is itself schematic and qsqc. In particular, the functor $f_{*}$, right adjoint to $f^{*}$, is continuous. Hence, the functor $f^{*}$ preserves compactness.

This implies that the pullback of every compact object $\mathcal{F} \in \mathrm{QCoh}(\mathrm{y})$ to every affine scheme $S$ is perfect, and hence dualizable as an object of the symmetric monoidal category $\mathrm{QCoh}(S)$.

Since

$$
\mathrm{QCoh}(\mathrm{y}) \simeq \lim _{S \rightarrow y} \mathrm{QCoh}(S)
$$

we obtain that $\mathcal{F}$ is dualizable in $\mathrm{QCoh}(\mathrm{y})$.

3.5.6. Consider the pullback of $\alpha$ along the map

$$
\iota: y^{\phi} \rightarrow y \text {. }
$$

Using the fact that $\iota=\phi \circ \iota$, we obtain a map

$$
\iota^{*}(\mathcal{F}) \stackrel{\alpha}{\rightarrow} \iota^{*} \circ \phi^{*}(\mathcal{F})=(\phi \circ \iota)^{*}(\mathcal{F}) \simeq \iota^{*}(\mathcal{F}) .
$$

This is an endomorphism of $\iota^{*}(\mathcal{F})$, which we denote $\alpha^{\phi}$. We claim:

Proposition 3.5.7. The element

$$
\operatorname{cl}(\mathcal{F}, \alpha) \in \operatorname{Tr}\left(\phi^{*}, \operatorname{QCoh}(y)\right) \simeq \Gamma\left(y^{\phi}, \mathcal{O}_{y \phi}\right)
$$

identifies with

$$
\operatorname{Tr}\left(\alpha^{\phi}, \iota^{*}(\mathcal{F})\right)
$$

where the latter trace is taken in the symmetric monoidal category QCoh $\left(y^{\phi}\right)$, and where we identify

$$
\operatorname{End}_{\mathrm{QCoh}\left(y_{\phi}\right)}\left(\mathbf{1}_{\mathrm{QCoh}\left(y_{\phi}\right)}\right)=\operatorname{End}_{\mathrm{QCoh}\left(y_{\phi}\right)}\left(\mathcal{O}_{y \phi}\right) \simeq \Gamma\left(y^{\phi}, \mathcal{O}_{y_{\phi}}\right) .
$$


This is proved in KP1, Prop. 2.2.3]; see Sect. 4.2 below for a proof in a more general context.

3.5.8. Let now $y$ be a quasi-compact algebraic stack with an affine diagonal over a ground field of characteristic 0 . Consider the category $D-\bmod (y)$.

In this case, the functors

$$
\operatorname{Vect} \stackrel{e \mapsto \omega y}{\longrightarrow} \mathrm{D}-\bmod (y) \stackrel{\Delta_{\mathrm{dR}} *}{\longrightarrow} \mathrm{D}-\bmod (y \times y) \simeq \mathrm{D}-\bmod (y) \otimes \mathrm{D}-\bmod (y)
$$

and

$$
\mathrm{D}-\bmod (y) \otimes \mathrm{D}-\bmod (y) \simeq \mathrm{D}-\bmod (y \times y) \stackrel{\Delta^{!}}{\longrightarrow} \mathrm{D}-\bmod (y) \stackrel{\Gamma_{\mathrm{dR}}(y,-)}{\longrightarrow} \operatorname{Vect}
$$

define an identification

$$
\operatorname{D}-\bmod (y)^{\vee} \simeq \operatorname{D}-\bmod (y)
$$

Let $\phi$ be an endomorphism of $\mathcal{F}$. Consider the endofunctor of $\mathrm{D}-\bmod (y)$, given by $\phi_{\mathrm{dR}, *}$. Then as in Sect. 3.5.3 one shows that there exists a canonical identification

$$
\operatorname{Tr}\left(\phi_{\mathrm{dR}, *}, \mathrm{D}-\bmod (y)\right) \simeq \Gamma_{\mathrm{dR}}\left(y^{\phi}, \omega_{\mathrm{y} \phi}\right),
$$

where the latter object is usually called the Borel-Moore homology of $y^{\phi}$.

3.5.9. Let $\mathcal{F}$ be a compact object of $D-\bmod (y)$ equipped with a map

$$
\alpha: \mathcal{F} \rightarrow \phi_{\mathrm{dR}, *}(\mathcal{F}) .
$$

To this object there corresponds an element

$$
\operatorname{cl}(\mathcal{F}, \alpha) \in \operatorname{Tr}\left(\phi_{\mathrm{dR}, *}, \mathrm{D}-\bmod (y)\right) \simeq \Gamma_{\mathrm{dR}}\left(y^{\phi}, \omega_{y \phi}\right) .
$$

However, we do not at the moment know how to give an explicit formula for this element, which would be reminiscent of that of Proposition 3.5 .7

However, according to $\mathrm{Va}$, the following particular case is known:

Theorem 3.5.10. Assume that $y$ is a scheme, and let $y \in y^{\phi}$ be an isolated fixed point. Assume, moreover, that $\left.d \phi\right|_{T_{y}(Y)}$ does not have eigenvalue 1 (i.e., the derived fixed point locus $y \phi$ is smooth at $y)$. Assume that $\mathcal{F}$ is holonomic. Then the image of $\operatorname{cl}(\mathcal{F}, \alpha)$ under the projection on the direct summand corresponding to $y$

$$
\Gamma_{\mathrm{dR}}\left(y^{\phi}, \omega_{y \phi}\right) \rightarrow \text { Vect }
$$

equals

$$
\operatorname{Tr}\left(\alpha^{\phi}, \iota_{y}^{\mathrm{dR}, *}(\mathcal{F})\right),
$$

where $\iota_{y}$ denotes the embedding $\mathrm{pt} \stackrel{y}{\rightarrow} y$, and $\alpha^{\phi}$ denotes the induced endomorphism of $\iota_{y}^{\mathrm{dR}, *}(\mathcal{F})$ equal to

$$
\iota_{y}^{\mathrm{dR}, *}(\mathcal{F}) \simeq\left(\phi \circ \iota_{y}\right)^{\mathrm{dR}, *}(\mathcal{F})=\iota_{y}^{\mathrm{dR}, *} \circ \phi^{\mathrm{dR}, *}(\mathcal{F}) \stackrel{\iota_{y}^{\mathrm{dR}, *}\left(\alpha^{\prime}\right)}{\longrightarrow} \iota_{y}^{\mathrm{dR}, *}(\mathcal{F})
$$

and where

$$
\alpha^{\prime}: \phi^{\mathrm{dR}, *}(\mathcal{F}) \rightarrow \mathcal{F}
$$

is obtained from $\alpha$ by the $\left(\phi^{\mathrm{dR}, *}, \phi_{\mathrm{dR}, *}\right)$-adjunction. 
3.5.11. A prototype for the moduli of shtukas. Here we generalize some aspects of the above example and relate it to the construction of the moduli space of shtukas (in the setting of D-modules in characteristic 0 ). Let $y$ be a quasi-compact algebraic stack with affine diagonal over a field of characteristic 0 . Suppose we have a "Frobenius" endomorphism

$$
\phi: y \rightarrow y \text {. }
$$

Moreover, suppose that we have a correspondence

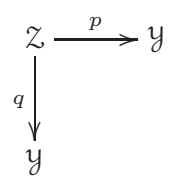

and a sheaf $K \in \mathrm{D}-\bmod (z)$. Let $H_{K}$ denote the functor

$$
H_{K}=q_{*}\left(p^{!}(-) \otimes K\right): \mathrm{D}-\bmod (y) \rightarrow \mathrm{D}-\bmod (y) .
$$

By the same considerations as above, we obtain

$$
\operatorname{Tr}\left(H_{K} \circ \phi_{*, \mathrm{dR}}, \mathrm{D}-\bmod (\mathrm{y})\right) \simeq \Gamma_{\mathrm{dR}}\left(z^{\phi}, i^{!}(K)\right)
$$

where $z^{\phi}$ is the pullback

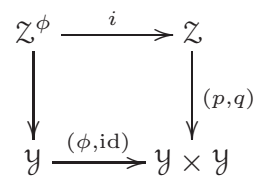

Analogously, consider $y=\operatorname{Bun}_{G}(X)$, where $X$ is an algebraic curve over a finite field $\mathbb{F}_{q}, \phi$ is the morphism induced by the Frobenius on $X, Z$ is the Hecke stack (of modifications at some legs $x^{I} \in X^{I}$ ) and $K$ is the Satake image of a representation of $G^{\vee}$. In this case, $Z^{\phi}$ is the moduli stack of shtukas and $\Gamma\left(Z^{\phi}, i^{!}(K)\right)$ are the corresponding cohomologies of interest on the moduli of shtukas.

\subsection{Trace on DG 2-categories.}

3.6.1. We will now study a different instance of the abstract formalism of Sect. 3.2.1 Namely, we take

$$
\mathbf{O}:=\operatorname{Morita}(\text { DGCat). }
$$

By definition, objects of this category are parameterized by $\mathcal{R} \in \mathrm{DGCat}^{\mathrm{Mon}}$. We will denote the corresponding object of Morita(DGCat) by $\underline{\mathcal{R}-\bmod }$.

For two objects $\underline{\mathcal{R}_{1}-\mathbf{m o d}}, \underline{\mathcal{R}_{2}-\bmod } \in \operatorname{Morita}(D G C a t)$, the $(\infty, 1)$-category of 1 -morphisms

$$
\operatorname{Maps}_{\text {Morita(DGCat) }}\left(\underline{\mathcal{R}_{1}-\mathbf{m o d}}, \underline{\mathcal{R}_{2}-\mathbf{m o d}}\right)
$$

is by definition

$$
\mathcal{R}_{2} \otimes \mathcal{R}_{1}^{\text {rev }}-\bmod \text {. }
$$

For an object $Q \in \mathcal{R}_{2} \otimes \mathcal{R}_{1}^{\text {rev }}$ - mod, we will denote the corresponding object of (3.16) by $\mathfrak{T}_{Q}$. The identity 1 -morphism is given by $\mathfrak{T}_{\mathcal{R}}$, where we view $\mathcal{R}$ as an $\mathcal{R} \otimes \mathcal{R}^{\text {rev }}$-module category.

The composition of two 1-morphisms

$$
\underline{\mathcal{R}_{1}-\bmod } \stackrel{\mathfrak{T}_{Q_{1,2}}}{\longrightarrow} \underline{\mathcal{R}_{2}-\bmod } \stackrel{\mathfrak{T}_{Q_{2,3}}}{\longrightarrow} \underline{\mathcal{R}_{3}-\bmod }
$$

is set to be $\mathfrak{T}_{Q_{1,3}}$, where

$$
\mathfrak{T}_{Q_{1,3}}:=\mathfrak{T}_{Q_{2,3}} \underset{\mathcal{R}_{2}}{\otimes} \mathfrak{T}_{Q_{1,2}} .
$$


3.6.2. Note that a 1-morphism

$$
\mathfrak{T}: \underline{\mathcal{R}_{1}-\bmod } \rightarrow \underline{\mathcal{R}_{2}-\bmod }
$$

defines a functor of 2-categories

$$
\mathcal{R}_{1}-\bmod \rightarrow \mathcal{R}_{2}-\bmod ,
$$

which, by a slight abuse of notation, we will denote by the same character $\mathfrak{T}$.

Namely, for $\mathfrak{T}=\mathfrak{T}_{Q}$, the functor 3.17) is given by

$$
\mathcal{M} \mapsto \mathcal{Q} \underset{\mathcal{R}_{1}}{\otimes} \mathcal{M} .
$$

Sometimes, we will refer to this construction as "evaluation of $\mathfrak{T}$ on $\mathcal{M}$ ".

Remark 3.6.3. We use Morita(DGCat) as defined above as a substitute for the $(\infty, 2)$-category of "2-DG categories", hence the title of this subsection.

Whatever the latter is, it contains Morita(DGCat) as a full subcategory, which consists of unigenerated 2-DG categories.

By way of analogy, Morita(Vect) is a full subcategory of DGCat that consists of DG categories that can be generated by a single compact object.

3.6.4. The symmetric monoidal structure on Morita(DGCat) is given by

$$
\underline{\mathcal{R}_{1}-\bmod } \otimes \underline{\mathcal{R}_{2}-\bmod }:=\underline{\left(\mathcal{R}_{1} \otimes \mathcal{R}_{2}\right)-\bmod } .
$$

Note that the unit object of Morita(DGCat) is

$$
\underline{\text { Vect }-\mathbf{m o d}}=\text { : DGCat. }
$$

3.6.5. Every object of Morita(DGCat) is dualizable. We have

$$
\underline{\mathcal{R}-\mathbf{m o d}^{\vee}} \simeq \underline{\mathcal{R}^{\text {rev }}-\bmod },
$$

where the unit and counit map are both given by

$$
\mathcal{R} \in\left(\mathcal{R} \otimes \mathcal{R}^{\text {rev }}\right)-\bmod \text {. }
$$

Under this identification, for a 1-morphism $\underline{\mathcal{R}_{1}-\mathbf{m o d}} \rightarrow \underline{\mathcal{R}_{2}-\bmod }$ corresponding to

$$
\mathcal{M} \in\left(\mathcal{R}_{1}^{\text {rev }} \otimes \mathcal{R}_{2}\right)-\bmod ,
$$

the dual 1-morphism $\underline{\mathcal{R}_{2}^{\text {rev }}-\bmod } \rightarrow \underline{\mathcal{R}_{1}^{\text {rev }}-\bmod }$ is given by the same $\mathcal{M}$.

3.6.6. We have

$$
\operatorname{End}_{\text {Morita(DGCat) }}\left(\mathbf{1}_{\text {Morita(DGCat) }}\right) \simeq \text { DGCat },
$$

as a symmetric monoidal category

Further,

$$
\operatorname{Maps}_{\text {Morita(DGCat) }}\left(\mathbf{1}_{\text {Morita(DGCat })}, \underline{\mathcal{R}-\mathbf{m o d}}\right) \simeq \mathcal{R}-\bmod
$$

3.6.7. Thus, we obtain that for every object $\mathfrak{C} \in$ Morita(DGCat) and its endomorphism $\mathfrak{F}$, we can attach

$$
\operatorname{Tr}(\mathfrak{F}, \mathfrak{C}) \in \text { DGCat }
$$

Furthermore, if

$$
\mathfrak{T}: \mathfrak{C}_{1} \rightarrow \mathfrak{C}_{2}
$$

is a 1-morphism that admits a right adjoint, and given

$$
\alpha: \mathfrak{T} \circ \mathfrak{F}_{1} \rightarrow \mathfrak{F}_{2} \circ \mathfrak{T},
$$

we obtain a functor

$$
\operatorname{Tr}(\mathfrak{T}, \alpha): \operatorname{Tr}\left(\mathfrak{F}_{1}, \mathfrak{C}_{1}\right) \rightarrow \operatorname{Tr}\left(\mathfrak{F}_{2}, \mathfrak{C}_{2}\right)
$$


3.6.8. Note that for $\mathcal{Q} \in \mathcal{R}_{2} \otimes \mathcal{R}_{1}^{\text {rev }}$ - $\bmod$ the condition that $\mathfrak{F}_{\mathcal{Q}}$ admit a right adjoint means that $\mathcal{Q}$ is right-dualizable as a bimodule category.

By definition, this means that there exists an object

$$
Q^{R} \in \mathcal{R}_{1} \otimes \mathcal{R}_{2}^{\text {rev }}-\mathbf{m o d}
$$

equipped the map

$$
\mathcal{R}_{1} \rightarrow \mathcal{Q}^{R} \underset{\mathcal{R}_{2}}{\otimes} \mathcal{Q}
$$

in $\left(\mathcal{R}_{1} \otimes \mathcal{R}_{1}^{\text {rev }}\right)$ - $\bmod$ and a map

$$
\mathcal{Q} \underset{\mathcal{R}_{1}}{\otimes} \mathcal{Q}^{R} \rightarrow \mathcal{R}_{2}
$$

in $\left(\mathcal{R}_{2} \otimes \mathcal{R}_{2}^{\text {rev }}\right)$ - $\bmod$ that satisfy the usual axioms.

3.6.9. The case of particular interest for us is when the 1-morphism

$$
\underline{\mathcal{R}_{2}-\bmod } \rightarrow \underline{\mathcal{R}_{1}-\bmod }
$$

is given by a monoidal functor

$$
\Psi: \mathcal{R}_{1} \rightarrow \mathcal{R}_{2},
$$

i.e., it is given by the $\left(\mathcal{R}_{1}, \mathcal{R}_{2}\right)$-bimodule $\mathcal{Q}_{\Psi}$, which is isomorphic to $\mathcal{R}_{2}$ as a DG category, on which $\mathcal{R}_{2}$ acts by right multiplication, and $\mathcal{R}_{1}$ acts by left multiplication via $\Psi$.

Denote this 1-morphism by $\operatorname{Res}_{\Psi}$. The corresponding functor

$$
\operatorname{Res}_{\Psi}: \mathcal{R}_{2}-\bmod \rightarrow \mathcal{R}_{1}-\bmod
$$

is given by restriction via $\Psi$.

The 1-morphism $\operatorname{Res}_{\Psi}$ tautologically admits a left adjoint, denoted $\operatorname{Ind}_{\Psi}$. It is given by the $\left(\mathcal{R}_{2}, \mathcal{R}_{1}\right)$ bimodule $\mathcal{R}_{2}$, on which $\mathcal{R}_{2}$ acts by left multiplication, and $\mathcal{R}_{1}$ acts by right multiplication via $\Psi$. The unit map for the adjunction is given by

$$
\mathcal{R}_{1} \underset{\Psi}{\rightarrow} \mathcal{R}_{2} \simeq \mathcal{R}_{2} \underset{\mathcal{R}_{2}}{\otimes} \mathcal{R}_{2}
$$

and the counit of the adjunction is given by the multiplication map

$$
\mathcal{R}_{2} \underset{\mathcal{R}_{1}}{\otimes} \mathcal{R}_{2} \rightarrow \mathcal{R}_{2}
$$

The corresponding functor

$$
\operatorname{Ind}_{\Psi}: \mathcal{R}_{1}-\bmod \rightarrow \mathcal{R}_{2}-\bmod
$$

is given by

$$
\mathcal{M} \mapsto \mathcal{R}_{2} \underset{\mathcal{R}_{1}}{\otimes} \mathcal{M}
$$

3.6.10. Assume now that $\mathcal{R}_{1}$ and $\mathcal{R}_{2}$ are rigid (see GR1, Chapter 1, Sect. 9.1]). In this case, the 1-morphism $\operatorname{Res}_{\Psi}$ admits also a right adjoint, denoted $\operatorname{coInd}_{\Psi}$.

The corresponding $\left(\mathcal{R}_{2}, \mathcal{R}_{1}\right)$-bimodule is again $\mathcal{R}_{2}$. The unit map for the adjunction.

$$
\mathcal{R}_{2} \rightarrow \mathcal{R}_{2} \underset{\mathcal{R}_{1}}{\otimes} \mathcal{R}_{2}
$$

is the right adjoint to the multiplication functor

$$
\mathcal{R}_{2} \underset{\mathcal{R}_{1}}{\otimes} \mathcal{R}_{2} \rightarrow \mathcal{R}_{2}
$$

(the right adjoint is a functor of $\mathcal{R}_{2}$-bimodule categories due to rigidity). The counit of the adjunction is the functor

$$
\mathcal{R}_{2} \underset{\mathcal{R}_{2}}{\otimes} \mathcal{R}_{2} \simeq \mathcal{R}_{2} \rightarrow \mathcal{R}_{1}
$$

right adjoint to $\Psi$ (again, this right adjoint is a functor of $\mathcal{R}_{1}$-bimodule categories due to rigidity).

Remark 3.6.11. Note that we obtain that in the situation when $\mathcal{R}_{1}$ and $\mathcal{R}_{2}$ are rigid, the left and right adjoints of $\operatorname{Res} \Psi$ are canonically isomorphic. 


\subsection{The 2-categorical trace and (categorical) Hochschild chains.}

3.7.1. For $\mathcal{R} \in \mathrm{DGCat}^{\mathrm{Mon}}$ and $\mathcal{Q} \in\left(\mathcal{R} \otimes \mathcal{R}^{\text {rev }}\right)$ - mod, denote

$$
\begin{gathered}
\mathrm{HH}_{\bullet}(\mathcal{R}, \mathcal{Q}):=\mathcal{R} \underset{\mathcal{R} \otimes \mathcal{R}^{\text {rev }}}{\otimes} \mathrm{Q} \in \text { DGCat; } \\
\mathrm{HH}_{\bullet}(\mathcal{R}):=\mathcal{R} \underset{\mathcal{R} \otimes \mathcal{R}^{\text {rev }}}{\otimes} \mathcal{R} .
\end{gathered}
$$

As in Sect. 3.5.1 we obtain formally that

$$
\operatorname{Tr}\left(\mathfrak{T}_{Q}, \underline{\mathcal{R}-\bmod }\right) \simeq \mathrm{HH}_{\bullet}(\mathcal{R}, Q) .
$$

3.7.2. Let $\mathcal{Q}$ be given by a monoidal endofunctor $F_{\mathcal{R}}$ of $\mathcal{R}$, see Sect. 3.6 .9 i.e., $\mathcal{Q}=Q_{F_{\mathcal{R}}}$. In this case, by a slight abuse of notation, we will write

$$
\mathrm{HH}_{\bullet}\left(\mathcal{R}, F_{\mathcal{R}}\right)
$$

instead of $\mathrm{HH}_{\bullet}\left(\mathcal{R}, \mathcal{Q}_{F_{\mathcal{R}}}\right)$.

3.7.3. Example. Let $\mathcal{R}=\mathrm{QCoh}(y)$ for $y$ as in Sect. 3.5 .2 and with affine diagona 11 , and let $F_{\mathcal{R}}$ be given by $\phi^{*}$ for an endomorphism $\phi$ of $y$.

By Corollary 1.6.5 we have:

$$
\mathrm{HH}_{\bullet}\left(\mathrm{QCoh}(y), \phi^{*}\right) \simeq \mathrm{QCoh}\left(y^{\phi}\right)
$$

3.7.4. Let us consider another example:

Let $\mathcal{A}$ be a symmetric monoidal category, and for a space $Y$ consider $\mathcal{A}^{\otimes Y}$. Let $\phi$ be an endomorphism of $Y$. By functoriality, it induces a symmetric monoidal functor

$$
\mathcal{A}^{\otimes \phi}: \mathcal{A}^{\otimes Y} \rightarrow \mathcal{A}^{\otimes Y} .
$$

Note that since the monoidal structures involved are symmetric, the category

$$
\mathrm{HH}_{\bullet}\left(\mathcal{A}^{\otimes Y}, \mathcal{A}^{\otimes \phi}\right)
$$

also acquires a symmetric monoidal structure.

Let $Y / \phi$ denote the quotient of $Y$ by $\phi$, i.e.,

$$
Y / \phi:=Y_{\text {id } \sqcup \mathrm{id}, Y \sqcup Y, \phi \sqcup \mathrm{id}}^{\sqcup} Y,
$$

where the subscripts indicate the morphisms with respect to which we form the pushout.

Note that when $\phi$ is an automorphism, $Y / \phi$ is isomorphic to the quotient $Y / \mathbb{Z}$, i.e., to the geometric realization of the bar simplicial space

$$
\ldots \mathbb{Z} \times Y \rightrightarrows Y
$$

We claim:

Proposition 3.7.5. There exists a canonical equivalence

$$
\mathrm{HH}_{\bullet}\left(\mathcal{A}^{\otimes Y}, \mathcal{A}^{\otimes \phi}\right) \simeq \mathcal{A}^{\otimes Y / \phi} .
$$

Proof. Follows from the commutation of the functor (1.3) with colimits in $Y$.

\footnotetext{
${ }^{11}$ One can show that the extra hypothesis of having an affine diagonal is not necessary here.
} 
3.7.6. Let us specialize further the example considered in Sect. 3.7.4 above.

Let e be a field of characteristic 0 , and take $\mathcal{A}=\operatorname{Rep}(\mathrm{G})$. Assume that $Y$ has finitely many connected components; then the same is true for $Y / \phi$.

Recall the identifications

$$
\left.\operatorname{Rep}(\mathrm{G})^{\otimes Y} \simeq \mathrm{QCoh} \operatorname{LocSys}_{\mathrm{G}}(Y)\right) \text { and } \operatorname{Rep}(\mathrm{G})^{\otimes Y / \phi} \simeq \mathrm{QCoh}\left(\operatorname{LocSys}_{\mathrm{G}}(Y / \phi)\right)
$$

of Theorem 1.5.5

With respect to the above identifications, the functor $\operatorname{Rep}(G)^{\otimes \phi}$ corresponds to the functor

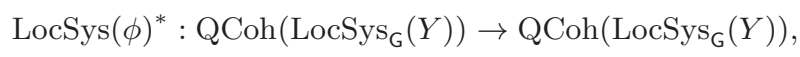

where

$$
\operatorname{LocSys}(\phi): \operatorname{LocSys}_{\mathrm{G}}(Y) \rightarrow \operatorname{LocSys}_{\mathrm{G}}(Y)
$$

is the map induced by $\phi$.

By Sect. 3.7.3. we have

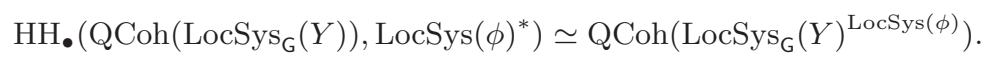

Now,

$$
\operatorname{LocSys}_{G}(Y)^{\operatorname{LocSys}(\phi)} \simeq \operatorname{LocSys}_{G}(Y / \phi) .
$$

To summarize, we obtain

$$
\begin{aligned}
\mathrm{HH}_{\bullet}\left(\operatorname{Rep}(\mathrm{G})^{\otimes Y}, \operatorname{Rep}(\mathrm{G})^{\otimes \phi}\right) & \simeq \mathrm{HH}_{\bullet}\left(\mathrm{QCoh}\left(\operatorname{LocSys}_{\mathrm{G}}(Y)\right), \operatorname{LocSys}(\phi)^{*}\right) \simeq \\
& \left.\simeq \mathrm{QCoh} \operatorname{LocSys}_{\mathrm{G}}(Y)^{\operatorname{LocSys}(\phi)}\right) \simeq \operatorname{QCoh}(\operatorname{LocSys}(Y / \phi)) \simeq \operatorname{Rep}(\mathrm{G})^{\otimes Y / \phi},
\end{aligned}
$$

which is what Proposition 3.7 .5 says in this case.

\subsection{The 2-categorical class map.}

3.8.1. Let $\mathcal{R}$ be a monoidal category, and let $\mathcal{M}$ be an $\mathcal{R}$-module. Assume that $\mathcal{M}$ is right-dualizable as an $\mathcal{R}$-module (see Sect. 3.6.8).

Then the corresponding functor

$$
\mathfrak{T}_{\mathcal{M}}: \underline{\mathrm{DGCat}} \rightarrow \underline{\mathcal{R}-\mathbf{m o d}}
$$

admits a right adjoint.

Let $\mathcal{Q}$ be an object of $\left(\mathcal{R} \otimes \mathcal{R}^{\text {rev }}\right)$ - mod, and let us be given a map

$$
\alpha: \mathcal{M} \rightarrow \underset{\mathcal{R}}{\otimes} \mathcal{M}
$$

in $\mathcal{R}-\bmod$.

Applying the functoriality of 2-categorical trace from 3.6.7 and repeating the construction of Sect. 3.4.3 to the above datum we can assign an object

$$
\operatorname{cl}(\mathcal{M}, \alpha) \in \operatorname{Tr}\left(\mathfrak{T}_{Q}, \underline{\mathcal{R}-\bmod }\right) \simeq \mathrm{HH}_{\bullet}(\mathcal{R}, \mathcal{Q}) .
$$


3.8.2. Note that for $\mathcal{M} \in \mathcal{R}-\mathbf{m o d}$ and $\mathcal{Q}=\mathcal{Q}_{F_{\mathcal{R}}}$ from Sect. 3.7 .2 the datum of $\alpha$ as in Sect. 3.8.1 amounts to an endofunctor $F_{\mathcal{M}}$, which is compatible with the action of $\mathcal{R}$ :

$$
F_{\mathcal{M}}(a \otimes m) \simeq F_{\mathcal{R}}(a) \otimes F_{\mathcal{M}}(m) .
$$

We denote this correspondence by

$$
F_{\mathcal{M}} \rightsquigarrow \alpha_{F_{\mathcal{M}}} .
$$

We will denote the corresponding object $\operatorname{cl}\left(\mathcal{M}, \alpha_{F_{\mathcal{M}}}\right) \in \mathrm{HH}_{\bullet}\left(\mathcal{R}, F_{\mathcal{R}}\right)$ also by

$$
\operatorname{Tr}_{\mathcal{R}}^{\mathrm{enh}}\left(F_{\mathcal{M}}, \mathcal{M}\right)
$$

The reason for the notation $\operatorname{Tr}_{\mathcal{R}}^{\text {enh }}$ will be explained in Remark 3.8.6 below.

Consider the particular case $\mathcal{M}=\mathcal{R}$ and $F_{\mathcal{M}}=F_{\mathcal{R}}$. We will denote

$$
\mathbf{1}_{\mathrm{HH} \bullet\left(\mathcal{R}, F_{\mathcal{R}}\right)}:=\operatorname{Tr}_{\mathcal{R}}^{\mathrm{enh}}\left(F_{\mathcal{R}}, \mathcal{R}\right) \in \mathrm{HH}_{\bullet}\left(\mathcal{R}, F_{\mathcal{R}}\right) .
$$

Remark 3.8.3. The reason for the notation $\mathbf{1}_{\mathrm{HH}}\left(\mathcal{R}, F_{\mathcal{R}}\right)$ is the following:

Assume for a moment that $\mathcal{R}$ is symmetric monoidal. The symmetric monoidal structure on $\mathcal{R}$

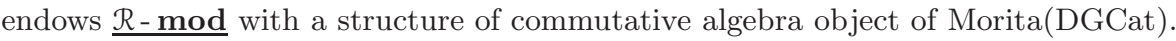

Assume that $F_{\mathcal{R}}$ is a symmetric monoidal endofunctor of $\mathcal{R}$. Consider the corresponding endomorphism $F_{\mathcal{R}}$ of $\underline{\mathcal{R}-\bmod }$ (see Sect. 3.7.2). This endomorphism is right-lax symmetric monoidal for the above commutative algebra structure on $\underline{\mathcal{R} \text { - mod. }}$. Hence, by Sect. 3.3 .2 , the category $\mathrm{HH}_{\bullet}\left(\mathcal{R}, F_{\mathcal{R}}\right)$ acquires a symmetric monoidal structure.

Now, it follows from (3.12) that the object that we have denoted

$$
\mathbf{1}_{\mathrm{HH} \bullet\left(\mathcal{R}, F_{\mathcal{R}}\right)} \in \mathrm{HH}_{\bullet}\left(\mathcal{R}, F_{\mathcal{R}}\right)
$$

is the monoidal unit.

3.8.4. We claim:

Theorem 3.8.5. Assume that $\mathcal{R}$ is rigid. Then there is a canonical isomorphism of associative algebras

$$
\operatorname{Tr}\left(F_{\mathcal{R}}, \mathcal{R}\right) \simeq \mathcal{E} n d_{\mathrm{HH}_{\bullet}\left(\mathcal{R}, F_{\mathcal{R}}\right)}\left(\mathbf{1}_{\mathrm{HH}_{\bullet}\left(\mathcal{R}, F_{\mathcal{R}}\right)}\right),
$$

and of modules over these algebras

$$
\operatorname{Tr}\left(F_{\mathcal{M}}, \mathcal{M}\right) \simeq \mathcal{H}^{\circ o m_{\mathrm{HH}}\left(\mathcal{R}, F_{\mathcal{R}}\right)}\left(\mathbf{1}_{\mathrm{HH}}\left(\mathcal{R}, F_{\mathcal{R}}\right), \operatorname{Tr}_{\mathcal{R}}^{\mathrm{enh}}\left(F_{\mathcal{M}}, \mathcal{M}\right)\right)
$$

Remark 3.8.6. The reason for the notation $\operatorname{Tr}_{\mathcal{R}}^{\mathrm{enh}}\left(F_{\mathcal{M}}, \mathcal{M}\right)$ is explained by Theorem 3.8.5 this theorem says that the object

$$
\operatorname{Tr}\left(F_{\mathcal{M}}, \mathcal{M}\right) \in \text { Vect }
$$

upgrades to an object of $\mathrm{HH}_{\bullet}\left(\mathcal{R}, F_{\mathcal{R}}\right)$ (namely, $\left.\operatorname{Tr}_{\mathcal{R}}^{\mathrm{enh}}\left(F_{\mathcal{M}}, \mathcal{M}\right)\right)$, where "upgrades" means that the former is the image of the latter under the functor

$$
\mathcal{H}^{\circ} m_{\mathrm{HH}_{\bullet}\left(\mathcal{R}, F_{\mathcal{R}}\right)}\left(\mathbf{1}_{\mathrm{HH}_{\bullet}\left(\mathcal{R}, F_{\mathcal{R}}\right)},-\right): \mathrm{HH}_{\bullet}\left(\mathcal{R}, F_{\mathcal{R}}\right) \rightarrow \text { Vect },
$$

which we can think of as a kind of forgetful functor.

Remark 3.8.7. It will follow from the proof that when $\mathcal{R}$ is symmetric monoidal, the isomorphism (3.20) that we will construct respects the commutative algebra structure on both sides. 
3.8.8. Example. Let $y$ be as in Sect. 3.5.2 and take $\mathcal{R}=\mathrm{QCoh}(y)$. Note the conditions on $y$ imply that $\mathrm{QCoh}(\mathrm{y})$ is rigid, see GR1, Chapter 3, Proposition 3.5.3]. We let $F_{\mathcal{R}}$ be given by $\phi^{*}$.

Let $\mathcal{M}$ be an QCoh(y)-module category, which is dualizable as a plain DG category. Let $F_{\mathcal{M}}$ be an endofunctor of $\mathcal{M}$ that is compatible with $\phi^{*}$, i.e., it makes the diagram

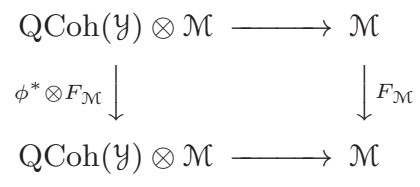

commute.

Then the construction of Sect. 3.8.1 produces an object

$$
\operatorname{Tr}_{\mathrm{QCoh}(y)}^{\mathrm{enh}}\left(F_{\mathcal{M}}, \mathcal{M}\right) \in \mathrm{QCoh}\left(y^{\phi}\right)
$$

and Theorem 3.8 .5 says that

$$
\Gamma\left(y^{\phi}, \operatorname{Tr}_{\mathrm{Q} \operatorname{Coh}(y)}^{\mathrm{enh}}\left(F_{\mathcal{M}}, \mathcal{M}\right)\right) \simeq \operatorname{Tr}\left(F_{\mathcal{M}}, \mathcal{M}\right)
$$

\subsection{A framework for the proof of Theorem 3.8.5.}

3.9.1. In order to prove Theorem 3.8.5 we will use the formalism of Sect. 3.3.1 applied to

$$
\mathbf{O}:=\operatorname{Morita}(\text { DGCat }), \operatorname{Endo}\left(\mathbf{1}_{\mathbf{O}}\right)=\text { DGCat } .
$$

However, we note that both sides in

$$
\operatorname{Tr}: L(\text { Morita(DGCat) })_{\text {rgd }} \rightarrow \text { DGCat }
$$

are naturally $(\infty, 2)$-categories, and functor (3.22) upgrades to a functor between $(\infty, 2)$-categories.

3.9.2. The structure of $(\infty, 2)$-category on $L$ (Morita(DGCat) $)_{\text {rgd }}$ comes from the structure of $(\infty, 3)$ category on Morita(DGCat).

Indeed, note that for a pair of objects $\underline{\mathcal{R}_{i}-\mathbf{m o d}} \in \operatorname{Morita}(\mathrm{DGCat})$, the $(\infty, 1)$-category

$$
\operatorname{Maps}_{\text {Morita (DGCat) }}\left(\underline{\mathcal{R}_{1}-\bmod }, \underline{\mathcal{R}_{2}-\bmod }\right):=\left(\mathcal{R}_{2} \otimes \mathcal{R}_{1}^{\text {op }}\right)-\bmod
$$

naturally upgrades to an $(\infty, 2)$-category. Namely, for

$$
Q^{\prime}, Q^{\prime \prime} \in\left(\mathcal{R}_{2} \otimes \mathcal{R}_{1}^{\text {op }}\right)-\bmod
$$

we can consider the $(\infty, 1)$-category

$$
\operatorname{Maps}_{\left(\mathcal{R}_{2} \otimes \mathcal{R}_{1}^{\text {op }}\right)-\bmod }\left(Q^{\prime}, Q^{\prime \prime}\right) .
$$

3.9.3. Let us specify what are the 2 -morphisms in $L$ (Morita(DGCat) $)_{\text {rgd }}$.

By definition, 1-morphisms in $L$ (Morita(DGCat) $)_{\text {rgd }}$ are diagrams

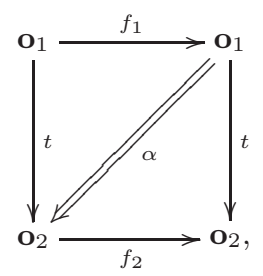

where $\mathbf{o}_{i} \in$ Morita(DGCat), and the edges in the above diagram are 1-morphisms in Morita(DGCat), where we require the 1 -morphism $t$ to admit a right adjoint. 
Given another 1-morphism represented by

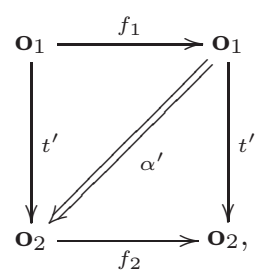

a 2-morphism between them is a diagram

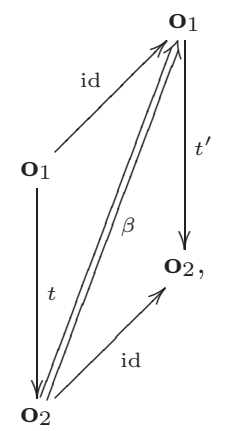

where $\beta: t \rightarrow t^{\prime}$ is a 2-morphism in Morita(DGCat) that admits a right adjoin 12, and which is equipped with a 3-morphism $\gamma$ for the cube:

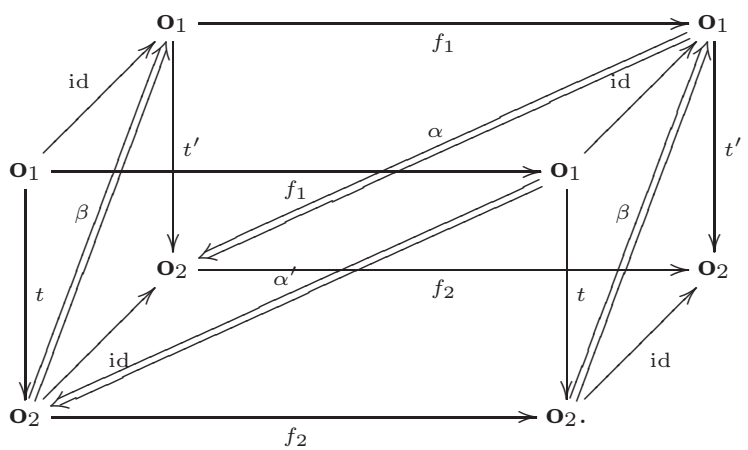

I.e., $\gamma$ is a 3 -morphism

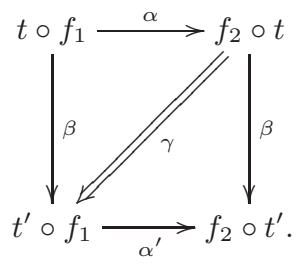

3.9.4. Let us show how the datum of $(\beta, \gamma)$ as above gives rise to a 2-morphism

$$
\operatorname{Tr}(t, \alpha) \rightarrow \operatorname{Tr}\left(t^{\prime}, \alpha^{\prime}\right)
$$

in

$$
\operatorname{End}_{\text {Morita(DGCat) }}(\underline{\text { DGCat }}) \simeq \text { DGCat },
$$

which is a 3-morphism in DGCat.

\footnotetext{
${ }^{12}$ This is a notion that exists in an $(\infty, 3)$-category.
} 
It is obtained from the diagram

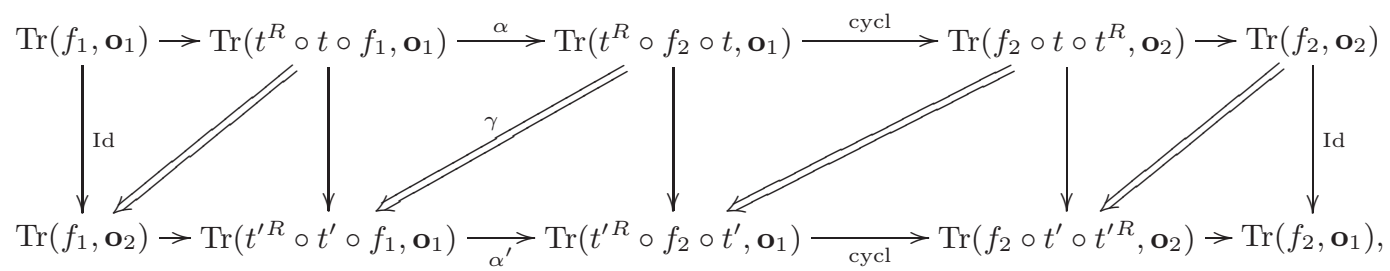

where the middle vertical arrows are induced by the 2-morphisms:

- $\beta: t \rightarrow t^{\prime}$

- The 2-morphism $t^{R} \rightarrow t^{\prime R}$, obtained by passing to right adjoints at the level of 1-morphisms from $\beta^{R}: t^{\prime} \rightarrow t$, i.e., we consider the partially defined functor "passing to the right adjoint"

$$
\operatorname{Maps}\left(\mathbf{o}_{2}, \mathbf{o}_{1}\right) \rightarrow \operatorname{Maps}\left(\mathbf{o}_{1}, \mathbf{o}_{2}\right), \quad t \mapsto t^{R}
$$

and apply it to

$$
\beta^{R} \in \operatorname{Maps}_{\operatorname{Maps}\left(\mathbf{o}_{2}, \mathbf{o}_{1}\right)}\left(t^{\prime}, t\right)
$$

to obtain an object of

$$
\operatorname{Maps}_{\operatorname{Maps}\left(\mathbf{o}_{1}, \mathbf{o}_{2}\right)}\left(t^{R}, t^{\prime R}\right) \text {. }
$$

\subsection{Proof of Theorem 3.8.5 isomorphism of the underlying objects of Vect.}

3.10.1. As a first step, in the setting of Theorem 3.8.5 we will show that we have a canonical isomorphism

$$
\operatorname{Tr}\left(F_{\mathcal{M}}, \mathcal{M}\right) \simeq \mathcal{H} \operatorname{som}_{\mathrm{HH}_{\bullet}\left(\mathcal{R}, F_{\mathcal{R}}\right)}\left(\mathbf{1}_{\mathrm{HH} \bullet}\left(\mathcal{R}, F_{\mathcal{R}}\right), \operatorname{Tr}_{\mathcal{R}}^{\mathrm{enh}}\left(F_{\mathcal{M}}, \mathcal{M}\right)\right)
$$

as objects of Vect.

We will do it in the following general framework.

3.10.2. Let $\mathcal{R}_{1}$ and $\mathcal{R}_{2}$ be a pair of monoidal DG categories, each equipped with a monoidal endofunctor, denoted $F_{\mathcal{R}_{1}}$ and $F_{\mathcal{R}_{2}}$, respectively. Let $\Psi: \mathcal{R}_{1} \rightarrow \mathcal{R}_{2}$ be a monoidal functor, equipped with an isomorphism

$$
F_{\mathcal{R}_{2}} \circ \Psi \simeq \Psi \circ F_{\mathcal{R}_{1}} .
$$

Restriction and induction along $\Psi$ define an adjoint pair of 1-morphisms in Morita(DGCat)

$$
\operatorname{Ind}_{\Psi}: \underline{\mathcal{R}_{1}-\bmod } \rightleftarrows \underline{\mathcal{R}_{2}-\bmod }: \operatorname{Res}_{\Psi},
$$

see Sect. 3.6.9.

The isomorphism (3.26) give rise to a commutative square

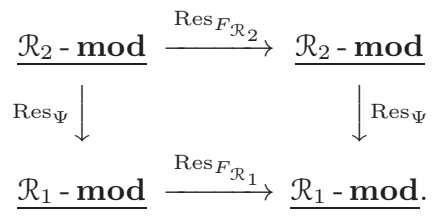


By passing to left adjoints along the vertical arrows in (3.28), we obtain a lax-commutative square: (i.e., a square that commutes up to a 2-morphism)

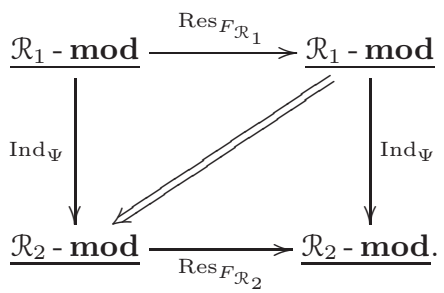

3.10.3. Assume now that $\mathcal{R}_{1}$ and $\mathcal{R}_{2}$ are rigid. In this case, by Sect. 3.6.10 the 1-morphism $\operatorname{Res}_{\Psi}$ admits also a right adjoint.

Furthermore, in this case the unit and the counit of the adjunction $\left(\operatorname{Ind}{ }_{\Psi}, \operatorname{Res} \Psi\right)$, which are 2morphisms

$$
\mathrm{Id} \rightarrow \operatorname{Res} \Psi_{\Psi} \circ \operatorname{Ind}_{\Psi} \text { and } \operatorname{Ind}_{\Psi} \circ \operatorname{Res}_{\Psi} \rightarrow \mathrm{Id}
$$

in Morita(DGCat), admit right adjoints.

Indeed, the above 2-morphisms are given by the functors

$$
\mathcal{R}_{1} \stackrel{\Psi}{\rightarrow} \mathcal{R}_{2} \text { and } \mathcal{R}_{2} \underset{\mathcal{R}_{1}}{\otimes} \mathcal{R}_{2} \stackrel{\text { mult }}{\rightarrow} \mathcal{R}_{2}
$$

(as $\left(\mathcal{R}_{1}, \mathcal{R}_{1}\right)$ - and $\left(\mathcal{R}_{2}, \mathcal{R}_{2}\right)$-bimodule categories-bimodule categories, respectively) and the rigidity assumption implies that these functors admit right adjoints that respect the bimodule structures.

From here it follows that the adjunction (3.27) and the diagrams (3.28) and (3.29) give rise to an adjunction

$$
\operatorname{Ind}_{\Psi}:\left(\underline{\mathcal{R}_{1}-\bmod }, \operatorname{Res}_{F_{\mathcal{R}_{1}}}\right) \rightleftarrows\left(\underline{\mathcal{R}_{2}-\bmod }, \operatorname{Res}_{F_{\mathcal{R}_{2}}}\right): \operatorname{Res}_{\Psi}
$$

in the $(\infty, 2)$-category $L$ (Morita(DGCat) $)_{\text {rgd }}$. In the unit and counit 2-morphisms that encode the adjunction (3.30), the corresponding 3-morphisms (denoted $\gamma$ in Sect. 3.9.3 see (3.24) ) are actually isomorphisms.

3.10.4. Hence, by Sect. 3.9, from the adjunction (3.30), by applying the functor Tr of (3.22), we obtain an adjunction

$$
\operatorname{Tr}\left(\operatorname{Ind}_{\Psi}\right): \mathrm{HH}_{\bullet}\left(\mathcal{R}_{1}, F_{\mathcal{R}_{1}}\right) \rightleftarrows \mathrm{HH}_{\bullet}\left(\mathcal{R}_{2}, F_{\mathcal{R}_{2}}\right): \operatorname{Tr}\left(\operatorname{Res}_{\Psi}\right)
$$

3.10.5. Let $\mathcal{M}$ be an object of $\mathcal{R}_{2}$ - mod; assume that it is dualizable as a DG category. Since $\mathcal{R}_{2}$ was assumed rigid, we obtain that $\mathcal{M}$ is dualizable as an object of $\mathcal{R}_{2}$ - mod.

Let $F_{\mathcal{M}}$ be its endofunctor as in Sect. 3.8.2 Then to it there corresponds an object

$$
\operatorname{Tr}_{\mathcal{R}_{2}}^{\mathrm{enh}}\left(F_{\mathcal{M}}, \mathcal{M}\right) \in \mathrm{HH}_{\bullet}\left(\mathcal{R}_{2}, F_{\mathcal{R}_{2}}\right) \text {. }
$$

Consider now the object $\operatorname{Res}_{\Psi}(\mathcal{M}) \in \mathcal{R}_{1}$ - mod. Since $\mathcal{R}_{1}$ is rigid and $\mathcal{M}$ is dualizable as a $\mathrm{DG}$ category, we obtain that $\operatorname{Res} \Psi(\mathcal{M})$ is dualizable as an object of $\mathcal{R}_{1}-\bmod$.

The datum of $F_{\mathcal{M}}$ defines the corresponding datum for $\operatorname{Res}_{\Psi}(\mathcal{M}) \in \mathcal{R}_{1}-\bmod$.

We will deduce Theorem 3.8.5 (at the level of the underlying objects of Vect) from the following more general relative statement:

Theorem 3.10.6. In the situation above, there is a canonical isomorphism

$$
\operatorname{Tr}(\operatorname{Ind} \Psi)^{R}\left(\operatorname{Tr}_{\mathcal{R}_{2}}^{\mathrm{enh}}\left(F_{\mathcal{M}}, \mathcal{M}\right)\right) \simeq \operatorname{Tr}_{\mathcal{R}_{1}}^{\mathrm{enh}}\left(F_{\mathcal{M}}, \operatorname{Res} \Psi(\mathcal{M})\right) .
$$


3.10.7. Proof of Theorem 3.10.6. We can view the pair $\left(\mathcal{M}, F_{\mathcal{M}}\right)$ as a 1 -morphism

$$
(\underline{\mathrm{DGCat}}, \mathrm{Id}) \rightarrow\left(\underline{\mathcal{R}_{2}-\bmod }, \operatorname{Res}_{F_{\mathcal{R}_{2}}}\right)
$$

in $L$ (Morita(DGCat) $)_{\mathrm{rgd}}$.

By construction,

$$
\operatorname{Tr}_{\mathcal{R}_{2}}^{\mathrm{enh}}\left(F_{\mathcal{M}}, \mathcal{M}\right) \in \operatorname{Tr}\left(\operatorname{Res}_{F_{\mathcal{R}_{2}}}, \underline{\mathcal{R}_{2}-\mathbf{m o d}}\right):=\mathrm{HH}_{\bullet}\left(\mathcal{R}_{2}, F_{\mathcal{R}_{2}}\right)
$$

is obtained by applying the functor $\operatorname{Tr}$ of 3.22 to (3.32).

The pair $\left(\operatorname{Res}_{\Psi}(\mathcal{M}), F_{\mathcal{M}}\right)$ is obtained from (3.32) as the composition

$$
(\underline{\mathrm{DGCat}}, \mathrm{Id}) \stackrel{\left(\mathcal{N}, F_{\Re}\right)}{\longrightarrow}\left(\underline{\mathcal{R}_{2}-\bmod }, \operatorname{Res}_{F_{\mathcal{R}_{2}}}\right) \stackrel{\operatorname{Res}_{\Psi}}{\longrightarrow}\left(\underline{\mathcal{R}_{1}-\bmod }, \operatorname{Res}_{F_{\mathcal{R}_{1}}}\right) .
$$

Hence,

$$
\operatorname{Tr}_{\mathcal{R}_{1}}^{\mathrm{enh}}\left(F_{\mathcal{M}}, \operatorname{Res} \Psi(\mathcal{M})\right) \in \operatorname{Tr}\left(\operatorname{Res}_{F_{\mathcal{R}_{1}}}, \underline{\mathcal{R}_{1}-\bmod }\right):=\mathrm{HH}_{\bullet}\left(\mathcal{R}_{1}, F_{\mathcal{R}_{1}}\right)
$$

is obtained by applying the functor $\operatorname{Tr}$ of 3.22 to (3.33).

3.10.8. From here we obtain a canonical identification

$$
\operatorname{Tr}\left(\operatorname{Res}_{\Psi}\right)\left(\operatorname{Tr}_{\mathcal{R}_{2}}^{\mathrm{enh}}\left(F_{\mathcal{M}}, \mathcal{M}\right)\right) \simeq \operatorname{Tr}_{\mathcal{R}_{1}}^{\mathrm{enh}}\left(F_{\mathcal{M}}, \operatorname{Res} \Psi(\mathcal{M})\right) .
$$

Note that by that by (3.31), we have:

$$
\operatorname{Tr}\left(\operatorname{Res}_{\Psi}\right) \simeq \operatorname{Tr}\left(\operatorname{Ind}_{\Psi}\right)^{R}
$$

Hence, we can rewrite 3.34 as:

$$
\operatorname{Tr}\left(\operatorname{Ind}_{\Psi}\right)^{R}\left(\operatorname{Tr}_{\mathcal{R}_{2}}^{\mathrm{enh}}\left(F_{\mathcal{M}}, \mathcal{M}\right)\right) \simeq \operatorname{Tr}_{\mathcal{R}_{1}}^{\mathrm{enh}}\left(F_{\mathcal{M}}, \operatorname{Res}_{\Psi}(\mathcal{M})\right)
$$

$\square[$ Theorem 3.10 .6

Remark 3.10.9. Note that for the construction of the equivalence 3.35 we use less than the full force of the assumption that both $\mathcal{R}_{1}$ and $\mathcal{R}_{2}$ be rigid. What we actually use is that the symmetric monoidal functor $\Psi: \mathcal{R}_{1} \rightarrow \mathcal{R}_{2}$ is rigid (we leave it to the reader to work out what this means).

For example, when $\mathcal{R}_{1}$ is symmetric monoidal and $\Psi$ makes $\mathcal{R}_{2}$ into a $\mathcal{R}_{1}$-algebra object in DGCat, the assumption we need is that $\mathcal{R}_{2}$ be rigid over $\mathcal{R}_{1}$. For $\mathcal{R}_{1}=$ Vect and $\mathcal{R}_{2}=\mathcal{R}$, this just means that $\mathcal{R}$ is rigid.

3.10.10. Let us now deduce the isomorphism (3.25). Take

$$
\left(\mathcal{R}_{2}, F_{\mathcal{R}_{2}}\right)=\left(\mathcal{R}, F_{\mathcal{R}}\right) \text { and }\left(\mathcal{R}_{1}, F_{\mathcal{R}_{1}}\right)=(\text { Vect }, \text { Id })
$$

with $\Psi$ being the unit functor Vect $\rightarrow \mathcal{R}$.

Note that by construction, the functor

$$
\operatorname{Tr}\left(\operatorname{Ind}_{\Psi}\right): \operatorname{Vect} \simeq \operatorname{Tr}(\operatorname{Id}, \underline{\mathrm{DGCat}}) \rightarrow \operatorname{Tr}\left(\operatorname{Res}_{F_{\mathcal{R}}}, \underline{\mathcal{R}-\bmod }\right):=\mathrm{HH}_{\bullet}\left(\mathcal{R}, F_{\mathcal{R}}\right)
$$

sends

see Remark 3.8 .3

$$
\mathrm{e} \mapsto \mathbf{1}_{\mathrm{HH}_{\bullet}\left(\mathcal{R}, F_{\mathcal{R}}\right)},
$$

Hence, $\operatorname{Tr}\left(\operatorname{Ind}_{\Psi}\right)^{R}$ is given by

$$
\left.\mathcal{H}^{\circ o m_{\mathrm{HH}}\left(\mathcal{R}, F_{\mathcal{R}}\right)}{ }_{\mathbf{1}_{\mathrm{HH}}\left(\mathcal{R}, F_{\mathcal{R}}\right)},-\right) .
$$

Finally, apply 3.35.

\subsection{Proof of Theorem 3.8.5; algebra and module structure.}


3.11.1. The functor $\operatorname{Tr}$ of 3.22 induces a functor

$$
\operatorname{Tr}: \operatorname{End}_{L(\operatorname{Morita}(\text { DGCat }))_{\mathrm{rgd}}}(\underline{\text { DGCat }}, \mathrm{Id}) \rightarrow \operatorname{End}_{\text {DGCat }}(\text { Vect }) \simeq \text { Vect } .
$$

We note that we have a canonical identification of symmetric monoidal categories

$$
L(\text { DGCat })_{\mathrm{rgd}} \simeq \operatorname{End}_{L(\operatorname{Morita}(\text { DGCat }))_{\mathrm{rgd}}}(\underline{\text { DGCat }}, \mathrm{Id}),
$$

and the resulting functor

$$
L(\text { DGCat })_{\text {rgd }} \rightarrow \text { Vect }
$$

is the functor (3.10) for $\mathbf{O}=$ DGCat.

3.11.2. Let $\Psi$ be the unit functor

$$
\text { DGCat } \rightarrow \mathcal{R}
$$

The adjunction

$$
\operatorname{Ind}_{\Psi}:(\underline{\text { DGCat }}, \mathrm{Id}) \leftrightarrows\left(\underline{\mathcal{R}-\bmod }, \operatorname{Res}_{F_{\mathcal{R}}}\right): \operatorname{Res} \Psi
$$

defines a monad on $(\underline{\text { DGCat }}, \mathrm{Id}) \in L(\text { Morita(DGCat) })_{\text {rgd }}$, i.e., an associative algebra in the (symmetric) monoidal category

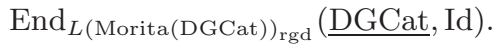

Under the identification (3.37) this algebra is given by

$$
\left(\mathcal{R}, F_{\mathcal{R}}\right) \in L(\text { DGCat })_{\text {rgd }} \text {. }
$$

Applying the functor $\operatorname{Tr}$ of (3.36) (which, by the above, is the same as the functor $\operatorname{Tr}$ of (3.10) for $\mathbf{O}=$ DGCat) we recover

with its associative algebra structure.

$$
\operatorname{Tr}\left(F_{\mathcal{R}}, \mathcal{R}\right) \in \text { Vect }
$$

3.11.3. Now, by the functoriality of (3.22), this associative algebra, regarded as a monad on Vect, identifies with the monad corresponding to the adjunction

$$
\operatorname{Tr}\left(\operatorname{Ind}_{\Psi}\right): \operatorname{Tr}((\underline{\mathrm{DGCat}}, \mathrm{Id})) \leftrightarrows \operatorname{Tr}\left(\left(\underline{\mathcal{R}-\bmod }, \operatorname{Res}_{F_{\mathcal{R}}}\right)\right): \operatorname{Tr}\left(\operatorname{Res}_{\Psi}\right)
$$

i.e., the adjunction obtained from (3.38) by applying the functor Tr.

We identify

$$
\operatorname{Tr}((\underline{\mathrm{DGCat}}, \mathrm{Id})) \simeq \text { Vect and } \operatorname{Tr}\left(\left(\underline{\mathcal{R}-\bmod }, \operatorname{Res}_{F_{\mathcal{R}}}\right)\right) \simeq \mathrm{HH}_{\bullet}\left(\mathcal{R}, F_{\mathcal{R}}\right),
$$

where the 1-morphism $\operatorname{Tr}\left(\operatorname{Ind}_{\Psi}\right)$ identifies with $\mathrm{e} \mapsto \mathbf{1}_{\mathrm{HH}_{\bullet}\left(\mathcal{R}, F_{\mathcal{R}}\right)}$.

Hence, we obtain that the associative algebra $\operatorname{Tr}\left(F_{\mathcal{R}}, \mathcal{R}\right) \in$ Vect corresponds to the adjunction

$$
\text { Vect } \leftrightarrows \mathrm{HH}_{\bullet}\left(\mathcal{R}, F_{\mathcal{R}}\right), \quad \mathrm{e} \mapsto \mathbf{1}_{\mathrm{HH}_{\bullet}\left(\mathcal{R}, F_{\mathcal{R}}\right)}
$$

This establishes the isomorphism (3.20) as associative algebras.

3.11.4. Similarly, a pair $\left(\mathcal{M}, F_{\mathcal{X}}\right)$ can be viewed as a 1-morphism

$$
(\underline{\text { DGCat }}, \mathrm{Id}) \rightarrow\left(\underline{\mathcal{R}-\bmod }, \operatorname{Res}_{F_{\mathcal{R}}}\right) \text {. }
$$

Composing with $\operatorname{Res}_{\Psi}$ we obtain an object in $\operatorname{End}_{L(\text { Morita(DGCat) })_{\mathrm{rgd}}}($ DGCat, Id), which is a module over the monad $\operatorname{Res}_{\Psi} \circ \operatorname{Ind}_{\Psi}$, and which under the identification (3.37) corresponds to

$$
\left(\mathcal{M}, F_{\mathcal{M}}\right) \in\left(\mathcal{R}, F_{\mathcal{R}}\right)-\bmod \left(L(\text { DGCat })_{\text {rgd }}\right) .
$$

Applying the functor $\operatorname{Tr}$ of (3.10) for $\mathbf{O}=$ DGCat, we recover $\operatorname{Tr}\left(F_{\mathcal{M}}, \mathcal{M}\right)$ as a module over $\operatorname{Tr}\left(F_{\mathcal{R}}, \mathcal{R}\right)$.

Now, by the functoriality of (3.22), the pair

$$
\operatorname{Tr}\left(F_{\mathcal{R}}, \mathcal{R}\right), \operatorname{Tr}\left(F_{\mathcal{M}}, \mathcal{M}\right) \in \operatorname{Tr}\left(F_{\mathcal{R}}, \mathcal{R}\right)-\bmod
$$

is the same as one obtained from

$$
\left(\mathcal{R}, F_{\mathcal{R}}\right),\left(\mathcal{M}, F_{\mathcal{M}}\right) \in\left(\mathcal{R}, F_{\mathcal{R}}\right)-\bmod \left(L(\text { DGCat })_{\mathrm{rgd}}\right)
$$

by applying the functor $\operatorname{Tr}$ of 3.22 . 
This establishes the isomorphism (3.21) as modules over the two sides of (3.20).

3.12. A more elementary proof of Theorem 3.8.5. For the convenience of the reader and future reference, in this subsection we will outline a more elementary proof (of a particular case) of Theorem 3.8.5 which does not use the machinery of $(\infty, 3)$-categories.

We first establish the stated isomorphism for the underlying objects of Vect.

3.12.1. Consider the following composition of 1-morphisms in Morita(DGCat)

$$
\underline{\text { DGCat }} \stackrel{\mathcal{M}}{\longrightarrow} \underline{\mathcal{R}-\bmod } \stackrel{\text { oblv }}{\longrightarrow} \underline{\text { DGCat }},
$$

where the second arrow is the forgetful map, i.e., given by $\mathfrak{T}_{\mathcal{R}}$. The composite is the map

$$
\underline{\text { DGCat }} \rightarrow \underline{\text { DGCat }}
$$

corresponding to oblv $(\mathcal{M}) \in$ DGCat, i.e., $\mathcal{M}$, viewed as a plain DG category.

We have the following diagram of 2-morphisms

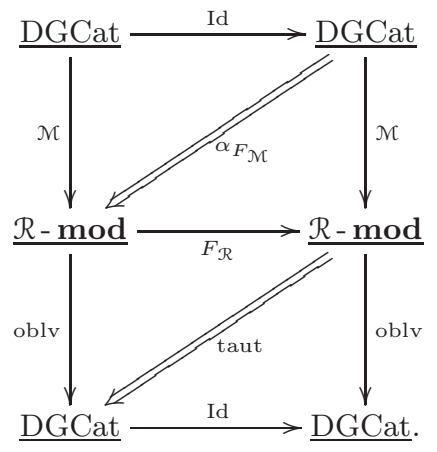

The composite 2-morphism identifies with

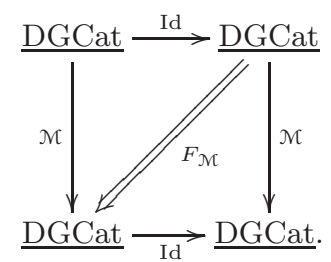

By unwinding the definitions, it is easy to see that the resulting map

$$
\operatorname{Tr}(\mathrm{Id}, \underline{\mathrm{DGCat}}) \rightarrow \operatorname{Tr}(\mathrm{Id}, \underline{\mathrm{DGCat}}),
$$

viewed as a functor

$$
\text { DGCat } \rightarrow \text { DGCat }
$$

is given by $\operatorname{Tr}\left(F_{\mathcal{M}}, \mathcal{M}\right) \in$ DGCat.

3.12.2. Hence, to prove the isomorphism (3.21) as vector spaces, it suffices to show that the map

$$
\operatorname{Tr}\left(F_{\mathcal{R}}, \underline{\mathcal{R}-\mathbf{m o d}}\right) \rightarrow \operatorname{Tr}(\mathrm{Id}, \underline{\text { DGCat }}),
$$


corresponding to the diagram

viewed as a functor

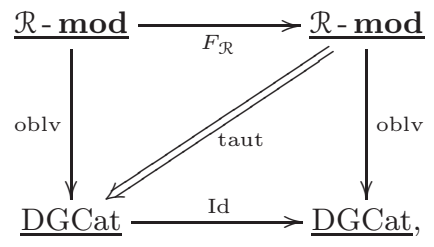

$$
\mathrm{HH}_{\bullet}\left(\mathcal{R}, F_{\mathcal{R}}\right) \rightarrow \text { Vect }
$$

is given by

$$
\mathcal{H}^{\circ o m_{\mathrm{HH}}\left(\mathcal{R}, F_{\mathcal{R}}\right)}\left(\mathbf{1}_{\mathrm{HH} \bullet\left(\mathcal{R}, F_{\mathcal{R}}\right)},-\right) .
$$

3.12.3. Consider the corresponding diagram

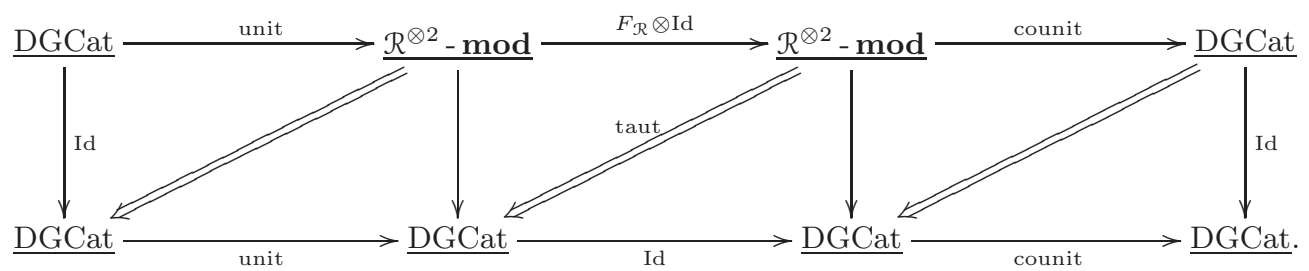

We need to calculate the 2-morphism from the clockwise circuit, which corresponds to

$$
\mathrm{HH}_{\bullet}\left(\mathcal{R}, F_{\mathcal{R}}\right) \in \mathrm{DGCat} \simeq \operatorname{End}(\underline{\text { DGCat }}),
$$

to the counterclockwise circuit, which corresponds to

$$
\text { Vect } \in \text { DGCat } \simeq \operatorname{End}(\underline{\text { DGCat }}) \text {. }
$$

3.12.4. We now recall that for a rigid symmetric monoidal category $\mathcal{R}^{\prime}$, the right adjoint to

$$
\underline{\mathcal{R}^{\prime}-\bmod } \stackrel{\text { oblv }}{\longrightarrow} \underline{\text { DGCat }}
$$

identifies with

$$
\underline{\text { DGCat }} \stackrel{\mathcal{R}^{\prime}}{\longrightarrow} \underline{\mathcal{R}^{\prime}-\mathbf{m o d}},
$$

see Sect. 3.6 .10

Thus, we obtain that the two middle vertical arrows in diagram (3.42) are given by the forgetful map

$$
\underline{(\mathcal{R} \otimes \mathcal{R})-\mathbf{m o d}} \stackrel{\text { oblv } \otimes \text { oblv }}{\longrightarrow} \underline{\text { DGCat }} .
$$

Furthermore, we obtain that the 2-morphism in the left square, viewed as a functor

$$
\mathcal{R} \rightarrow \text { Vect, }
$$

is given by

$$
\mathcal{H}_{\mathrm{om}}\left(\mathbf{1}_{\mathcal{R}},-\right) \text {. }
$$

The 2-morphism in the right square, evaluated on $\mathcal{Q} \in(\mathcal{R} \otimes \mathcal{R})$ - $\bmod$ (see Sect. 3.6.2 for what we mean by "evaluate"), is the map

$$
\mathrm{HH}_{\bullet}(\mathcal{R}, \mathcal{Q})=\mathcal{R} \underset{\mathcal{R} \otimes \mathcal{R}}{\otimes} \stackrel{\operatorname{mult}^{R} \otimes \operatorname{Id}_{\mathcal{Q}}}{\longrightarrow}(\mathcal{R} \otimes \mathcal{R}) \underset{\mathcal{R} \otimes \mathcal{R}}{\otimes} \mathrm{Q} \simeq \mathcal{Q},
$$


which is the right adjoint to the map

$$
\mathcal{Q} \simeq(\mathcal{R} \otimes \mathcal{R}) \underset{\mathcal{R} \otimes \mathcal{R}}{\otimes} \stackrel{\operatorname{mult} \operatorname{Id}_{\mathcal{Q}}}{\longrightarrow} \mathcal{R} \underset{\mathcal{R} \otimes \mathcal{R}}{\otimes} \mathrm{Q}=\mathrm{HH}_{\bullet}(\mathcal{R}, \mathcal{Q})
$$

3.12.5. We obtain that the functor in (3.40) equals the composite

$$
\mathrm{HH}_{\bullet}\left(\mathcal{R}, F_{\mathcal{R}}\right)=\mathcal{R} \underset{\text { mult }, \mathcal{R} \otimes \mathcal{R}, \text { mult } \circ\left(F_{\mathcal{R}} \otimes \mathrm{Id}\right)}{\otimes} \mathcal{R} \stackrel{(\text { mult })^{R} \otimes \mathrm{Id}}{\longrightarrow}(\mathcal{R} \otimes \mathcal{R}) \underset{\mathcal{R} \otimes \mathcal{R}, \text { mult } \circ\left(F_{\mathcal{R}} \otimes \mathrm{Id}\right)}{\otimes} \mathcal{R} \simeq \mathcal{R} \stackrel{\mathcal{H o m}_{\mathcal{R}}\left(\mathbf{1}_{\mathcal{R}},-\right)}{\longrightarrow} \text { Vect }
$$

By adjunction, this composite is the same as

i.e., 3.41).

$$
\mathrm{HH} \bullet\left(\mathcal{R}, F_{\mathcal{R}}\right) \stackrel{\mathcal{H}^{\circ} m_{\mathrm{HH}}\left(\mathcal{R}, F_{\mathcal{R}}\right)}{\longrightarrow} \stackrel{\left((\operatorname{mult} \otimes \mathrm{Id})\left(\mathbf{1}_{\mathcal{R}}\right),-\right)}{\longrightarrow} \text { Vect }
$$

Remark 3.12.6. Let us contrast the computation of the map (3.40) with the computation of the map

$$
\operatorname{Tr}(\mathrm{Id}, \mathrm{QCoh}(y)) \rightarrow \mathrm{e}
$$

corresponding to the functor

$$
\Gamma(y,-): \operatorname{QCoh}(y) \rightarrow \operatorname{Vect}
$$

where $y$ is a smooth proper scheme.

We identity

see Sect. 4.3 .3 .

$$
\operatorname{Tr}(\mathrm{Id}, \mathrm{QCoh}(\mathrm{y})) \simeq \underset{i}{\oplus} \Gamma\left(y, \Omega^{i}(y)\right)[i]
$$

The computation performed in [KP2] amounts to saying that the resulting map

$$
\underset{i}{\oplus} \Gamma\left(y, \Omega^{i}(y)\right)[i] \rightarrow \mathrm{e}
$$

is the projection

$$
\underset{i}{\oplus} \Gamma\left(y, \Omega^{i}(y)\right)[i] \rightarrow \Gamma\left(y, \Omega^{\text {top }}(y)\right)[\text { top }] \stackrel{\text { Serre duality }}{\longrightarrow} \text { e, }
$$

precomposed with the operation of multiplication by the Todd class. So, it is highly non-trivial. (3.41).

By contrast, in the setting of Sect. 3.12.2 the map 3.40 is something very simple, namely, the map

This my be viewed as an incarnation of the fact that for a rigid symmetric monoidal category, the 2 -category $\mathcal{R}$ - $\bmod$ is 0 -Calabi-Yau, in the sense that the left and right adjoints to the functor

$$
\underline{\text { DGCat }} \rightarrow \underline{\mathcal{R}-\mathbf{m o d}}, \quad \mathcal{C} \mapsto \mathcal{R} \otimes \mathcal{C}
$$

are canonically isomorphic, see Remark 3.6.11

3.12.7. Thus, we have established the isomorphism between the two sides of (3.21) as objects of Vect. In particular, we obtain an isomorphism between the two sides of (3.20), also as objects of Vect.

We will now assume that $\mathcal{R}$ is symmetric monoidal, and upgrade these isomorphisms to isomorphisms of algebras (resp., modules over them). This will be achieved by an Eckmann-Hilton argument.

Let $\mathbf{Q}$ denote the category, whose objects are quadruples

$$
\left(\mathcal{R}, \mathcal{M}, F_{\mathcal{R}}, F_{\mathcal{M}}\right)
$$

where $\mathcal{R}$ is a rigid symmetric monoidal DG category, and $\mathcal{M}$ is an $\mathcal{R}$-module, dualizable as a plain DG category.

For a pair of objects $\left(\mathcal{R}, \mathcal{M}, F_{\mathcal{R}}, F_{\mathcal{M}}\right)$ and $\left(\mathcal{R}^{\prime}, \mathcal{M}^{\prime}, F_{\mathcal{R}^{\prime}}, F_{\mathcal{M}^{\prime}}\right)$, the space of morphisms between them consists of a symmetric monoidal functor $\varphi_{\mathcal{R}}: \mathcal{R} \rightarrow \mathcal{R}^{\prime}$, intertwining $F_{\mathcal{R}}$ with $F_{\mathcal{R}^{\prime}}$, and a functor of $\mathcal{R}$-module categories $\varphi_{\mathcal{X}}: \mathcal{M} \rightarrow \mathcal{M}^{\prime}$, intertwining $F_{\mathcal{M}}$ with $F_{\mathcal{M}^{\prime}}$, such that the induced functor

$$
\mathcal{R}^{\prime} \underset{\mathcal{R}}{\otimes} \mathcal{M} \rightarrow \mathcal{M}^{\prime}
$$

is an equivalence. 
The assignments

$$
\left(\mathcal{R}, \mathcal{M}, F_{\mathcal{R}}, F_{\mathcal{X}}\right) \mapsto \operatorname{Tr}\left(F_{\mathcal{M}}, \mathcal{M}\right)
$$

and

$$
\left(\mathcal{R}, \mathcal{M}, F_{\mathcal{R}}, F_{\mathcal{M}}\right) \mapsto \mathcal{H} \operatorname{Hom}_{\mathrm{HC}}\left(\mathcal{R}, F_{\mathcal{R}}\right)\left(\mathbf{1}_{\mathrm{HC}}\left(\mathcal{R}, F_{\mathcal{R}}\right), \operatorname{Tr}_{\mathcal{R}}^{\mathrm{enh}}\left(F_{\mathcal{M}}, \mathcal{M}\right)\right)
$$

are both functors $\mathbf{Q} \rightarrow$ Vect.

Moreover, the category $\mathbf{Q}$ carries a naturally defined symmetric monoidal structure:

$$
\left(\mathcal{R}^{1}, \mathcal{M}^{1}, F_{\mathcal{R}^{1}}, F_{\mathcal{M}^{1}}\right) \otimes\left(\mathcal{R}^{2}, \mathcal{M}^{2}, F_{\mathcal{R}^{2}}, F_{\mathcal{M}^{2}}\right):=\left(\mathcal{R}^{1} \otimes \mathcal{R}^{2}, \mathcal{M}^{1} \otimes \mathcal{M}^{2}, F_{\mathcal{R}^{1}} \otimes F_{\mathcal{R}^{2}}, F_{\mathcal{M}^{1}} \otimes F_{\mathcal{M}^{2}}\right),
$$

and functors (3.46) and (3.47) are symmetric monoidal.

Furthermore, by the construction of the isomorphism of (3.21), it upgrades to an isomorphism of symmetric monoidal functors (3.46) and (3.47), as symmetric monoidal functors.

Note now that

$$
\left(\mathcal{R}, \mathcal{R}, F_{\mathcal{R}}, F_{\mathcal{R}}\right)
$$

is naturally a commutative algebra in Q. Hence, both sides of 3.20 have a structure of associative algebras, and (3.20) respects these structures.

By construction, the above commutative algebra structure on $\operatorname{Tr}\left(F_{\mathcal{R}}, \mathcal{R}\right)$ is the same one as given by the construction of Sect.3.4.4 Furthermore, by the Eckmann-Hilton argument, the above commutative algebra structure on

goes over under the forgetful functor

$$
\mathcal{E} n d_{\mathrm{HH}_{\bullet}\left(\mathcal{R}, F_{\mathcal{R}}\right)}\left(\mathbf{1}_{\mathrm{HH}_{\bullet}\left(\mathcal{R}, F_{\mathcal{R}}\right)}\right)
$$

$$
\text { ComAlg }(\text { Vect }) \rightarrow \text { AssocAlg(Vect), }
$$

to the structure of associative algebra on $\mathcal{E} n d$.

This implies the assertion that (3.20) is an algebra isomorphism.

3.12.8. Finally, for $\left(\mathcal{M}, F_{\mathcal{M}}\right)$ as in Sect. 3.8 .2 the object

$$
\left(\mathcal{R}, \mathcal{M}, F_{\mathcal{R}}, F_{\mathcal{M}}\right) \in \mathbf{Q}
$$

is a module over the algebra object $\left(\mathcal{R}, \mathcal{R}, F_{\mathcal{R}}, F_{\mathcal{R}}\right)$. The construction in Sect. 3.12.7 gives each side of (3.21) a structure of module over the corresponding side of (3.20), and (3.21) respects these structures.

The resulting action of $\operatorname{Tr}\left(F_{\mathcal{R}}, \mathcal{R}\right)$ on $\operatorname{Tr}\left(F_{\mathcal{N}}, \mathcal{M}\right)$ is the same one as given by the construction of Sect. 3.4.4 Again, by the Eckmann-Hilton argument, the resulting action of

$$
\mathcal{E} n d_{\mathrm{HH}_{\bullet}\left(\mathcal{R}, F_{\mathcal{R}}\right)}\left(\mathbf{1}_{\mathrm{HH}_{\bullet}\left(\mathcal{R}, F_{\mathcal{R}}\right)}\right)
$$

on

$$
\mathcal{H}_{o m_{\mathrm{HH}}\left(\mathcal{R}, F_{\mathcal{R}}\right)}\left(\mathbf{1}_{\mathrm{HH} \bullet}\left(\mathcal{R}, F_{\mathcal{R}}\right), \operatorname{Tr}_{\mathcal{R}}^{\mathrm{enh}}\left(F_{\mathcal{M}}, \mathcal{M}\right)\right)
$$

coincides with one coming from the action of $\mathcal{E}$ nd on $\mathcal{H}$ om.

This implies that (3.21) is an isomorphism of modules, as desired.

\section{A FEW MIND-TWISTERS}

In this section we will study some particular cases and generalizations of Theorem 3.8.5 We recommend the reader to skip this section on the first pass, because the assertions contained therein may appear abstract and un-motivated, and return to it when necessary.

That said, the results discussed in this section will all acquire a transparent meaning in the context of shtukas, which will be introduced in Sect. 5.

\subsection{The class of a class.}


4.1.1. Let $\mathcal{R}$ be a rigid symmetric monoidal category, and let $F_{\mathcal{R}}: \mathcal{R} \rightarrow \mathcal{R}$ be a symmetric monoidal endofunctor.

Consider the category

$$
\mathrm{HH}_{\bullet}\left(\mathcal{R}, F_{\mathcal{R}}\right):=\mathcal{R} \underset{\text { mult }, \mathcal{R} \otimes \mathcal{R}, \text { mult o }\left(F_{\mathcal{R}} \otimes \mathrm{Id}\right)}{\otimes} \mathcal{R} .
$$

Since the tensor product in the right-hand side of (4.1) involves symmetric monoidal categories and functors, we obtain that $\mathrm{HH}_{\bullet}\left(\mathcal{R}, F_{\mathcal{R}}\right)$ acquires a symmetric monoidal structure.

On the other hand, by (3.18), we have

$$
\mathrm{HH}_{\bullet}\left(\mathcal{R}, F_{\mathcal{R}}\right) \simeq \operatorname{Tr}\left(\operatorname{Res}_{F_{\mathcal{R}}}, \underline{\mathcal{R}-\mathbf{m o d}}\right)
$$

and hence it acquires a symmetric monoidal structure by Sect. 3.3 .2 .

However, it is easy to see that these two ways of defining a symmetric monoidal structure on $\mathrm{HH}_{\bullet}\left(\mathcal{R}, F_{\mathcal{R}}\right)$ are equivalent.

4.1.2. Denote by $\iota$ the functor

$$
\mathcal{R} \rightarrow \mathcal{R} \underset{\text { mult }, \mathcal{R} \otimes \mathcal{R}, \text { mult } \circ\left(F_{\mathcal{R}} \otimes \mathrm{Id}\right)}{\otimes} \mathcal{R} \simeq \mathrm{HH}_{\bullet}\left(\mathcal{R}, F_{\mathcal{R}}\right),
$$

corresponding to the left copy of $\mathcal{R}$. By (4.1), the functor $\iota$ is symmetric monoidal.

Note that by construction

$$
\iota \circ F_{\mathcal{R}} \simeq \iota
$$

4.1.3. Let $r \in \mathcal{R}$ be a compact object equipped with a map

$$
\alpha_{r}: r \rightarrow F_{\mathcal{R}}(r)
$$

On the one hand, to the pair $\left(r, \alpha_{r}\right)$ we attach its class

$$
\operatorname{cl}\left(r, \alpha_{r}\right) \in \operatorname{Tr}\left(F_{\mathcal{R}}, \mathcal{R}\right) .
$$

4.1.4. The data of $\alpha_{r}$ gives rise to a map

$$
\iota(r) \stackrel{\alpha_{r}}{\longrightarrow} \iota \circ F_{\mathcal{R}}(r) \stackrel{4.2}{\simeq} \iota(r)
$$

denote this map by $a_{r}^{F_{\mathcal{R}}}$.

Since $r \in \mathcal{R}$ is compact and $\mathcal{R}$ is rigid, we obtain that $r$ is dualizable as an object of $\mathcal{R}$ as a monoidal category. Since $\iota$ is symmetric monoidal, we obtain that $\iota(r)$ is dualizable as an object of $\mathrm{HH}_{\bullet}\left(\mathcal{R}, F_{\mathcal{R}}\right)$.

So, on the other hand, we can consider the element

$$
\operatorname{Tr}\left(a_{r}^{F_{\mathcal{R}}}, \iota(r)\right) \in \mathcal{E} n d_{\mathrm{HH}_{\bullet}\left(\mathcal{R}, F_{\mathcal{R}}\right)}\left(\mathbf{1}_{\mathrm{HH}}\left(\mathcal{R}, F_{\mathcal{R}}\right)\right) .
$$

4.1.5. We claim:

Proposition 4.1.6. The elements $\mathrm{cl}\left(r, \alpha_{r}\right)$ and $\operatorname{Tr}\left(a_{r}^{F_{\mathcal{R}}}, \iota(r)\right)$ coincide under the identification

$$
\operatorname{Tr}\left(F_{\mathcal{R}}, \mathcal{R}\right) \simeq \mathcal{E} n d_{\mathrm{HH}_{\bullet}\left(\mathcal{R}, F_{\mathcal{R}}\right)}\left(\mathbf{1}_{\mathrm{HH} \bullet\left(\mathcal{R}, F_{\mathcal{R}}\right)}\right)
$$

of Theorem 3.8 .5

Remark 4.1.7. Note that in the particular case of $\mathcal{R}=\mathrm{QCoh}(y)$ for a prestack $y$ as in Sect. 3.5.2 and $F_{\mathcal{R}}$ given by $\phi^{*}$ for an endomorphism $\phi$ of $y$, the assertion of Proposition 4.1.6. coincides with that of Proposition 3.5 .7

4.2. Proof of Proposition 4.1.6. The proof is a word-for-word repetition of the proof of KP1, Proposition 2.2.3]. We include it for the sake of completeness. 
4.2.1. First, let us make the isomorphism (4.3) explicit (this will imitate the manipulation in Sect. 3.5.3).

Recall that $\mathcal{R}$ is self-dual as a DG category, with the duality datum given by

$$
\text { Vect } \stackrel{\mathbf{1}_{\mathcal{R}}}{\rightarrow} \mathcal{R} \stackrel{\operatorname{mult}^{R}}{\longrightarrow} \mathcal{R} \otimes \mathcal{R}
$$

and

$$
\mathcal{R} \otimes \mathcal{R} \stackrel{\text { mult }}{\longrightarrow} \mathcal{R} \stackrel{\mathcal{H o m}_{\mathcal{R}}\left(\mathbf{1}_{\mathcal{R}},-\right)}{\longrightarrow} \text { Vect . }
$$

Hence, on the one hand, $\operatorname{Tr}\left(F_{\mathcal{R}}, \mathcal{R}\right)$ is the composition

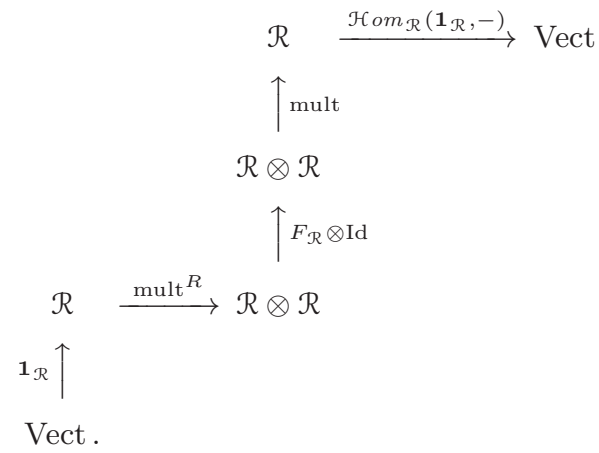

Consider the commutative diagram

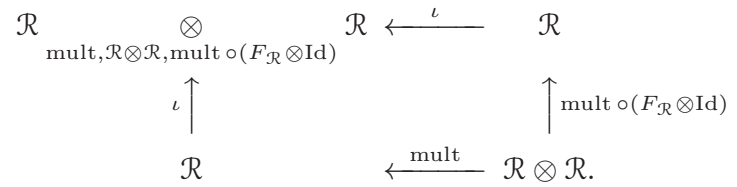

By rigidity, the diagram obtained by passing to right adjoints along the horizontal arrows is also commutative:

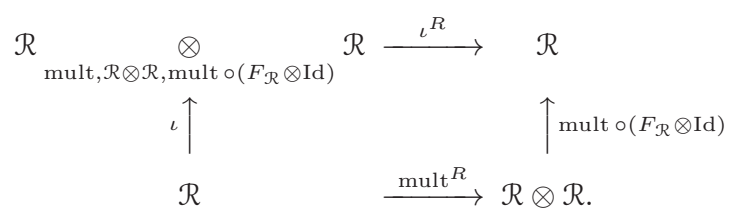

Hence, the composite in (4.4) identifies with

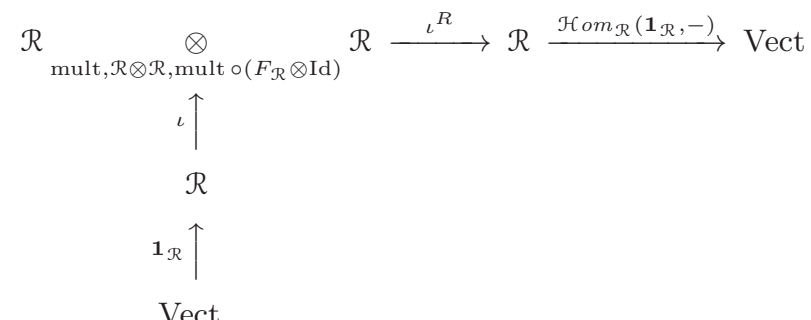

In the latter diagram, the composite horizontal arrow is the right adjoint of the composite vertical arrow, and the latter is

$$
\mathrm{e} \mapsto \mathbf{1}_{\mathrm{HH}_{\bullet}\left(\mathcal{R}, F_{\mathcal{R}}\right)} \in \mathrm{HH}_{\bullet}\left(\mathcal{R}, F_{\mathcal{R}}\right) \simeq \mathcal{R} \underset{\text { mult }, \mathcal{R} \otimes \mathcal{R}, \text { mult } \circ\left(F_{\mathcal{R}} \otimes \mathrm{Id}\right)}{\otimes} \mathcal{R} .
$$

Hence, the resulting functor Vect $\rightarrow$ Vect is given by $\mathcal{E} n d_{\mathrm{HH}\left(\mathcal{R}, F_{\mathcal{R}}\right)}\left(\mathbf{1}_{\mathrm{HH}\left(\mathcal{R}, F_{\mathcal{R}}\right)}\right)$. 
By unwinding the constructions (see Sect. 3.12), one shows that the identification

$$
\operatorname{Tr}\left(F_{\mathcal{R}}, \mathcal{R}\right) \simeq \mathcal{E} n d_{\mathrm{HH}_{\bullet}\left(\mathcal{R}, F_{\mathcal{R}}\right)}\left(\mathbf{1}_{\mathrm{HH}_{\bullet}\left(\mathcal{R}, F_{\mathcal{R}}\right)}\right)
$$

just constructed is equivalent to one in Theorem 3.8 .5

4.2.2. For a compact object $r \in \mathcal{R}$, let $r^{\vee} \in \mathcal{R}$ be its monoidal dual; this is also its formal dual with respect to the identification $\mathcal{R}^{\vee} \simeq \mathcal{R}$.

The class $\operatorname{cl}\left(r, \alpha_{r}\right) \in \operatorname{Tr}\left(F_{\mathcal{R}}, \mathcal{R}\right)$ corresponds to the 2-morphism from the clockwise circuit to the counter-clockwise circuit in the following diagram

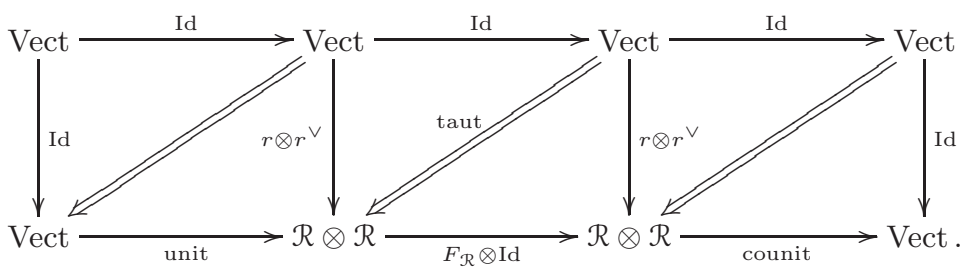

In this diagram, the 2-morphism in the left square is the map

$$
r \otimes r^{\vee} \rightarrow \operatorname{unit}(\mathrm{e})=\operatorname{mult}^{R}\left(\mathbf{1}_{\mathcal{R}}\right)
$$

equal to

$$
r \otimes r^{\vee} \rightarrow \operatorname{mult}{ }^{R} \circ \operatorname{mult}\left(r \otimes r^{\vee}\right)=\operatorname{mult}^{R}\left(r \otimes r^{\vee}\right) \rightarrow \operatorname{mult}^{R}\left(\mathbf{1}_{\mathcal{R}}\right)
$$

The 2-morphism in the middle square is obtained from the map

$$
\alpha_{r}: r \rightarrow F_{\mathcal{R}}(r) .
$$

The 2-morphism in the right square is map

$$
\mathrm{e} \rightarrow \operatorname{counit}\left(r \otimes r^{\vee}\right)=\mathcal{H}_{o} m_{\mathcal{R}}\left(\mathbf{1}_{\mathcal{R}}, \operatorname{mult}\left(r \otimes r^{\vee}\right)\right)=\mathcal{H}_{o} m_{\mathcal{R}}\left(\mathbf{1}_{\mathcal{R}}, r \otimes r^{\vee}\right),
$$

corresponding to the canonical map

$$
\mathbf{1}_{\mathcal{R}} \rightarrow r \otimes r^{\vee}
$$

4.2.3. Hence, $\operatorname{cl}\left(r, \alpha_{r}\right) \in \operatorname{Tr}\left(F_{\mathcal{R}}, \mathcal{R}\right)$ is the composite

$$
\begin{aligned}
\mathrm{e} \stackrel{\text { unit }}{\rightarrow} \mathcal{H} \operatorname{com}_{\mathcal{R}}\left(\mathbf{1}_{\mathcal{R}}, r \otimes r^{\vee}\right) \stackrel{\alpha_{r} \otimes \text { id }}{\longrightarrow} \mathcal{H} \operatorname{Com}_{\mathcal{R}}\left(\mathbf{1}_{\mathcal{R}}, F_{\mathcal{R}}(r) \otimes r^{\vee}\right) \simeq \\
\quad \simeq \mathcal{H} \operatorname{com}_{\mathcal{R}}\left(\mathbf{1}_{\mathcal{R}}, \operatorname{mult} \circ\left(F_{\mathcal{R}} \otimes \operatorname{Id}\right)\left(r \otimes r^{\vee}\right)\right) \rightarrow \mathcal{H} \operatorname{com}_{\mathcal{R}}\left(\mathbf{1}_{\mathcal{R}}, \text { mult } \circ\left(F_{\mathcal{R}} \otimes \text { Id }\right) \circ \text { mult }^{R}\left(\mathbf{1}_{\mathcal{R}}\right)\right) .
\end{aligned}
$$

Using the commutative diagram (4.6), we identify

$$
\text { mult } \circ\left(F_{\mathcal{R}} \otimes \mathrm{Id}\right) \circ \text { mult }^{R} \simeq \iota^{R} \circ \iota .
$$

Hence, we can rewrite the composition in (4.9) as

$$
\begin{aligned}
& \mathrm{e} \stackrel{\text { unit }}{\rightarrow} \mathcal{H} o m_{\mathcal{R}}\left(\mathbf{1}_{\mathcal{R}}, r \otimes r^{\vee}\right) \stackrel{\alpha_{r} \otimes \text { id }}{\longrightarrow} \mathcal{H} o m_{\mathcal{R}}\left(\mathbf{1}_{\mathcal{R}}, F_{\mathcal{R}}(r) \otimes r^{\vee}\right) \simeq \\
& \simeq \mathcal{H}_{o m_{\mathcal{R}}}\left(\mathbf{1}_{\mathcal{R}}, \text { mult } \circ\left(F_{\mathcal{R}} \otimes \mathrm{Id}\right)\left(r \otimes r^{\vee}\right)\right) \rightarrow \mathcal{H}_{o m_{\mathcal{R}}}\left(\mathbf{1}_{\mathcal{R}}, \text { mult } \circ\left(F_{\mathcal{R}} \otimes \mathrm{Id}\right) \circ \operatorname{mult}^{R}\left(r \otimes r^{\vee}\right)\right) \simeq \\
& \simeq \mathcal{H}_{\circ} \circ m_{\mathcal{R}}\left(\mathbf{1}_{\mathcal{R}}, \iota^{R} \circ \iota\left(r \otimes r^{\vee}\right)\right) \rightarrow \mathcal{H}^{\circ} \circ m_{\mathcal{R}}\left(\mathbf{1}_{\mathcal{R}}, \iota^{R} \circ \iota\left(\mathbf{1}_{\mathcal{R}}\right)\right) \text {. }
\end{aligned}
$$


4.2.4. We have a commutative diagram

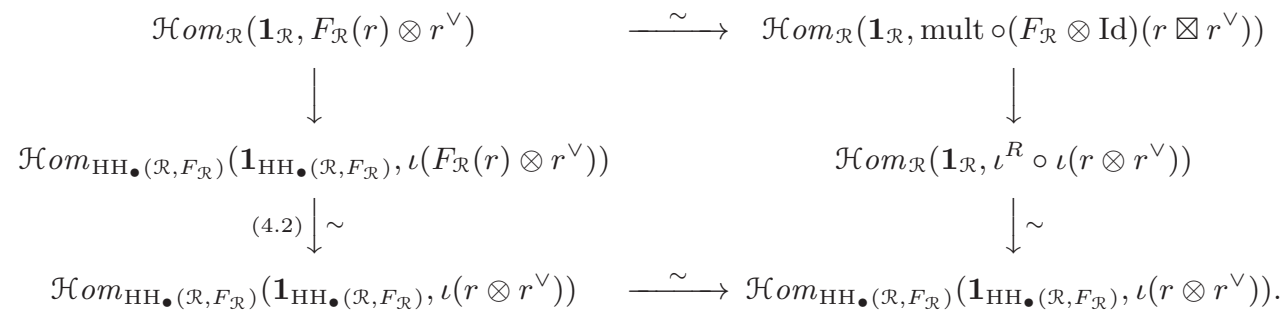

Hence, the composition in (4.10) can be rewritten as

$$
\begin{aligned}
& \mathrm{e} \stackrel{\text { unit }}{\rightarrow} \mathcal{H} o m_{\mathcal{R}}\left(\mathbf{1}_{\mathcal{R}}, r \otimes r^{\vee}\right) \stackrel{\alpha_{r} \otimes \text { id }}{\longrightarrow} \mathcal{H} o m_{\mathcal{R}}\left(\mathbf{1}_{\mathcal{R}}, F_{\mathcal{R}}(r) \otimes r^{\vee}\right) \rightarrow \\
& \rightarrow \mathcal{H}^{\circ o m_{\mathrm{HH}}\left(\mathcal{R}, F_{\mathcal{R}}\right)}\left(\mathbf{1}_{\mathrm{HH}}\left(\mathcal{R}, F_{\mathcal{R}}\right), \iota\left(F_{\mathcal{R}}(r) \otimes r^{\vee}\right)\right) \stackrel{[4.2}{\simeq} \mathcal{H}_{o m_{\mathrm{HH}}\left(\mathcal{R}, F_{\mathcal{R}}\right)}\left(\mathbf{1}_{\mathrm{HH}\left(\mathcal{R}, F_{\mathcal{R}}\right)}, \iota\left(r \otimes r^{\vee}\right)\right) \stackrel{\text { counit }}{\rightarrow}
\end{aligned}
$$

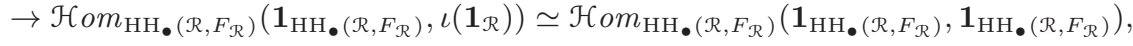

and further as

$$
\begin{aligned}
& \mathrm{e} \stackrel{\text { unit }}{\rightarrow} \mathcal{H} \mathcal{H}_{\mathrm{HH}_{\bullet}\left(\mathcal{R}, F_{\mathcal{R}}\right)}\left(\mathbf{1}_{\mathrm{HH}_{\bullet}\left(\mathcal{R}, F_{\mathcal{R}}\right)}, \iota(r) \otimes \iota\left(r^{\vee}\right)\right) \stackrel{\alpha_{r} \otimes \mathrm{id}}{\longrightarrow} \mathcal{H} \mathcal{H}_{\mathrm{HH}_{\bullet}\left(\mathcal{R}, F_{\mathcal{R}}\right)}\left(\mathbf{1}_{\mathrm{HH}_{\bullet}\left(\mathcal{R}, F_{\mathcal{R}}\right)}, \iota\left(F_{\mathcal{R}}(r)\right) \otimes \iota\left(r^{\vee}\right)\right) \stackrel{4.2}{\simeq} \\
& \simeq \mathcal{H}_{\operatorname{Com}_{\mathrm{HH}_{\bullet}\left(\mathcal{R}, F_{\mathcal{R}}\right)}}\left(\mathbf{1}_{\mathrm{HH}_{\bullet}\left(\mathcal{R}, F_{\mathcal{R}}\right)}, \iota(r) \otimes \iota\left(r^{\vee}\right)\right) \stackrel{\text { counit }}{\rightarrow} \mathcal{H}_{o m_{\mathrm{HH}_{\bullet}\left(\mathcal{R}, F_{\mathcal{R}}\right)}}\left(\mathbf{1}_{\mathrm{HH}_{\bullet}\left(\mathcal{R}, F_{\mathcal{R}}\right)}, \mathbf{1}_{\mathrm{HH}_{\bullet}\left(\mathcal{R}, F_{\mathcal{R}}\right)}\right),
\end{aligned}
$$

while the latter is the right-hand side is by definition $\operatorname{Tr}\left(a_{r}^{F_{\mathcal{R}}}, \iota(r)\right)$.

4.3. The "trivial" case and excursions. We will now specialize further to the case when $F_{\mathcal{R}}$ is the identity map.

4.3.1. Let us take $\alpha$ to be the identity endomorphism of $r$. Consider the corresponding endomorphism

$$
\operatorname{id}_{r}^{\operatorname{Id}_{\mathcal{R}}} \in \mathcal{E} n d_{\mathrm{HH}_{\bullet}(\mathcal{R})}(\iota(r)),
$$

see Sect. 4.1.4

Denote

$$
\operatorname{ch}(r):=\operatorname{Tr}\left(\operatorname{id}_{r}^{\operatorname{Id}_{\mathcal{R}}}, \iota(r)\right) \in \mathcal{E} n d_{\mathrm{HH}_{\bullet}(\mathcal{R})}\left(\mathbf{1}_{\mathrm{HH}_{\bullet}(\mathcal{R})}\right) .
$$

Note that according to Proposition 4.1.6 we have

$$
\operatorname{cl}\left(r, \mathrm{id}_{r}\right)=\operatorname{ch}(r)
$$

under the identification

$$
\operatorname{Tr}\left(\operatorname{Id}_{\mathcal{R}}, \mathcal{R}\right) \simeq \mathcal{E} n d_{\mathrm{HH}_{\bullet}(\mathcal{R})}\left(\mathbf{1}_{\mathrm{HH}_{\bullet}(\mathcal{R})}\right)
$$

of Theorem 3.8 .5

Remark 4.3.2. We emphasize that despite the fact that we plugged in the identity map everywhere, the endomorphism $\operatorname{id}_{r}^{\operatorname{Id}_{\mathcal{R}}}$ of $\iota(r)$ is not the identity map (for one thing, if it were the identity, formula (4.12) would fail).

See also the last line in the proof of Proposition 4.3.5 for another interpretation of the element $\mathrm{id}_{r}^{\mathrm{Id}_{\mathcal{R}}}$. 
4.3.3. An example. Let $\mathcal{R}=\mathrm{QCoh}(y)$, where $y$ is as in Sect. 3.5.2 In KP2 Sect. 1.2] it is explained that for $r=\mathcal{F} \in \mathrm{QCoh}(\mathrm{y})$, the map $\operatorname{id}_{r}^{\mathrm{Id}_{\mathcal{R}}}$ can be interpreted as the action of

$$
\mathcal{L}(y):=y \underset{y \times y}{\times} y
$$

thought of as the group object over $y$, on $\mathcal{F}$. Furthermore,

$$
\operatorname{ch}(r) \in \Gamma\left(\mathcal{L}(y), \mathcal{O}_{\mathcal{L}(y)}\right)
$$

can be identified with the character of this action.

Let now $y$ be a smooth scheme over a field of characteristic zero. Consider the derivative of the above action of $\mathcal{L}(y)$ on $\mathcal{F}$, which is an action of the Lie algebra

$$
\operatorname{Lie}(\mathcal{L}(Y)) \simeq T_{y}[-1]
$$

on $\mathcal{F}$. It is shown in $\mathrm{KP} 2$, Sect. 1.3] that the resulting map

$$
T y[-1] \otimes \mathcal{F} \rightarrow \mathcal{F}
$$

is the Atiyah class of $\mathcal{F}$.

Further, the Hochschild-Kostant-Rosenberg theorem identifies

$$
\Gamma\left(\mathcal{L}(y), \mathcal{O}_{\mathcal{L}(y)}\right) \simeq \oplus_{i} \Gamma\left(y, \Omega^{i}(y)\right)[i] .
$$

Under this identification, the element $\operatorname{ch}(r)$ corresponds to the classical Chern character

$$
\operatorname{ch}(\mathcal{F}) \in \underset{i}{\oplus} \Gamma\left(y, \Omega^{i}(y)\right)[i] .
$$

4.3.4. We will now give one more interpretation of the above element

$$
\operatorname{ch}(r) \in \mathcal{E} n d_{\mathrm{HH}_{\bullet}(\mathcal{R})}\left(\mathbf{1}_{\mathrm{HH}_{\bullet}(\mathcal{R})}\right),
$$

in terms of excursion operators.

Take

$$
Y=S^{1}=\{*\} \underset{\{*\} \sqcup\{*\}}{\sqcup}\{*\}
$$

so that

$$
\mathcal{R}^{\otimes S^{1}} \simeq \mathrm{HH}_{\bullet}(\mathcal{R})
$$

Consider the tautological point $\gamma_{\text {taut }} \in \Omega\left(S^{1}, *\right)$. Let $\gamma_{\text {taut }}^{+}$denote the pair $\left(\gamma_{\text {taut }}, \gamma_{\text {triv }}\right)$.

Let $\xi_{r}$ be the map

$$
\mathbf{1}_{\mathcal{R}} \rightarrow \text { mult } \circ \operatorname{mult}^{R}\left(\mathbf{1}_{\mathcal{R}}\right)
$$

equal to the composition

$$
\mathbf{1}_{\mathcal{R}} \rightarrow r \otimes r^{\vee} \simeq \operatorname{mult}\left(r \otimes r^{\vee}\right) \rightarrow \operatorname{mult} \circ \operatorname{mult}^{R}\left(\mathbf{1}_{\mathcal{R}}\right),
$$

where:

-the map $\mathbf{1}_{\mathcal{R}} \rightarrow r \otimes r^{\vee}$ is the unit of the duality;

$-r \otimes r^{\vee}$ denotes the corresponding object of $\mathcal{R} \otimes \mathcal{R}$;

-the map $r \otimes r^{\vee} \rightarrow \operatorname{mult}^{R}\left(\mathbf{1}_{\mathcal{R}}\right)$ is obtained by adjunction from the map

$$
\operatorname{mult}\left(r \otimes r^{\vee}\right)=: r \otimes r^{\vee} \stackrel{\text { counit }}{\longrightarrow} \mathbf{1}_{\mathcal{R}}
$$

We claim:

Proposition 4.3.5. The element

$$
\operatorname{ch}(r) \in \mathcal{E n d}_{\mathrm{HH}_{\bullet}(\mathcal{R})}\left(\mathbf{1}_{\mathrm{HH}(\mathcal{R})}\right)
$$

corresponds to the universal excursion element (see Sect. 2.8.4)

$$
\operatorname{Exc}_{\text {univ }}\left(\gamma_{\text {taut }}^{+}, \xi_{r}\right) \in \mathcal{E} n d_{\mathcal{R} \otimes S^{1}}\left(\mathbf{1}_{\mathcal{R} \otimes S^{1}}\right)
$$

under the identification 4.13). 
Proof. The proof is essentially an application of the definitions:

By formula (2.16), the element $\operatorname{Exc}_{\text {univ }}\left(\gamma_{\text {taut }}^{+}, \xi_{r}\right)$ is the composition

$$
\mathbf{1}_{\mathrm{HH}}(\mathcal{R}) \stackrel{\text { unit }}{\longrightarrow} \iota(r) \otimes \iota\left(r^{\vee}\right) \stackrel{\text { mon }_{\gamma_{\text {taut }}} \otimes \mathrm{id}}{\longrightarrow} \iota(r) \otimes \iota\left(r^{\vee}\right) \stackrel{\text { counit }}{\longrightarrow} \mathbf{1}_{\mathrm{HH}_{\bullet}(\mathcal{R})},
$$

where mon $_{\gamma_{\text {taut }}}$ denotes the automorphism of the functor $\iota$, i.e.,

$$
\mathcal{R} \simeq \mathcal{R}^{\otimes\{*\}} \rightarrow \mathcal{R}^{\otimes S^{1}},
$$

corresponding to the loop $\gamma_{\text {taut }}$.

However, by definition, the above automorphism $\operatorname{mon}_{\gamma_{\text {taut }}}$ of $\iota$, when evaluated on $r \in \mathcal{R}$, identifies with $\operatorname{id}_{r}^{\operatorname{Id}_{\mathcal{R}}}$.

Combining Propositions 4.1.6 and 4.3.5. we obtain:

Corollary 4.3.6. Under the identification

$$
\operatorname{Tr}(\mathrm{Id}, \mathcal{R}) \simeq \mathcal{E} n d_{\mathrm{HH}_{\bullet}(\mathcal{R})}\left(\mathbf{1}_{\mathrm{HH}(\mathcal{R})}\right) \simeq \mathcal{E} n d_{\mathcal{R}_{S^{1}}}\left(\mathbf{1}_{\mathcal{R} \otimes S^{1}}\right)
$$

of Theorem 3.8.5, the element

$$
\operatorname{cl}(r, \mathrm{id}) \in \operatorname{Tr}(\mathrm{Id}, \mathcal{R})
$$

goes over to

$$
\operatorname{Exc}_{\text {univ }}\left(\gamma_{\text {taut }}^{+}, \xi_{r}\right) \in \mathcal{E} n d_{\mathcal{R} \otimes S^{1}}\left(\mathbf{1}_{\mathcal{R} \otimes S^{1}}\right) .
$$

Remark 4.3.7. Note that on the one hand, $\operatorname{Tr}\left(\operatorname{Id}_{\mathcal{R}}, \mathcal{R}\right)$ is explicitly given by the composition

$$
\text { Vect } \stackrel{1_{\mathcal{R}}}{\rightarrow} \mathcal{R} \stackrel{\text { mult }^{R}}{\longrightarrow} \mathcal{R} \otimes \mathcal{R} \stackrel{\text { mult }}{\longrightarrow} \mathcal{R} \stackrel{\mathcal{H}^{\circ} m_{\mathcal{R}}\left(\mathbf{1}_{\mathcal{R}},-\right)}{\rightarrow} \text { Vect }
$$

i.e., the resulting vector space is

$$
\left.\mathcal{H}_{\mathrm{om}} \boldsymbol{1}_{\mathcal{R}}, \text { mult } \circ \text { mult }^{R}\left(\mathbf{1}_{\mathcal{R}}\right)\right) .
$$

On the other hand, by Lemma 2.7.4 we have

$$
\begin{aligned}
& \mathcal{E} n d_{\mathrm{HH}_{\bullet}(\mathcal{R})}\left(\mathbf{1}_{\mathrm{HH}_{\bullet}(\mathcal{R})}\right) \simeq \mathcal{E} n d_{\mathcal{R} \otimes S^{1}}\left(\mathbf{1}_{\mathcal{R} \otimes S^{1}}\right)=\mathcal{H}_{\mathcal{R}} m_{\mathcal{R} \otimes S^{1}}\left(\iota\left(\mathbf{1}_{\mathcal{R}}\right), \iota\left(\mathbf{1}_{\mathcal{R}}\right)\right) \simeq \\
& \simeq \mathcal{H}_{\circ} m_{\mathcal{R}}\left(\mathbf{1}_{\mathcal{R}}, \iota^{R} \circ \iota\left(\mathbf{1}_{\mathcal{R}}\right)\right) \simeq \mathcal{H} \circ m_{\mathcal{R}}\left(\mathbf{1}_{\mathcal{R}}, \text { mult } \circ \text { mult }^{R}\left(\mathbf{1}_{\mathcal{R}}\right)\right) .
\end{aligned}
$$

Thus, we obtain an identification

$$
\operatorname{Tr}\left(\operatorname{Id}_{\mathcal{R}}, \mathcal{R}\right) \simeq \mathcal{H} \operatorname{om}_{\mathcal{R}}\left(\mathbf{1}_{\mathcal{R}}, \text { mult } \circ \operatorname{mult}^{R}\left(\mathbf{1}_{\mathcal{R}}\right)\right) \simeq \mathcal{E} n d_{\mathrm{HH}_{\bullet}(\mathcal{R})}\left(\mathbf{1}_{\mathrm{HH}}(\mathcal{R})\right)
$$

By unwinding the definitions, one can show that this is the same identification as one given by Theorem 3.8.5

Assuming this, one can obtain the assertion of Corollary 4.3.6 by combining 4.12 and Theorem 2.8.7. Indeed, this follows from the fact that the element $\operatorname{cl}\left(r, \mathrm{id}_{r}\right)$, thought of as an element in

$$
\operatorname{Tr}\left(\operatorname{Id}_{\mathcal{R}}, \mathcal{R}\right) \simeq \mathcal{H}_{\mathcal{R}} m_{\mathcal{R}}\left(\mathbf{1}_{\mathcal{R}}, \text { mult } \circ \operatorname{mult}^{R}\left(\mathbf{1}_{\mathcal{R}}\right)\right),
$$

equals $\xi_{r}$.

4.4. Introducing observables. In this subsection we will study a certain generalization of Theorem 3.8.5 where we modify both sides by inserting an object $r \in \mathcal{R}$.

4.4.1. Let $\mathcal{R}$ be a rigid symmetric monoidal category. Let $\mathcal{M}$ be a dualizable $\mathcal{R}$-module category.

On the one hand, we consider the endofunctor of $\mathcal{M}$, given by $H_{r} \circ F_{\mathcal{M}}$, where

$$
H_{r}(m):=r \otimes m
$$

denotes the action of the object $r$ on $\mathcal{M}$ as an $\mathcal{R}$-module category. 
4.4.2. On the other hand, consider

$$
\iota(r) \in \mathrm{HH}_{\bullet}\left(\mathcal{R}, F_{\mathcal{R}}\right),
$$

where we recall that $\iota$ denotes the functor

$$
\mathcal{R} \rightarrow \mathcal{R} \underset{\text { mult }, \mathcal{R} \otimes \mathcal{R}, \text { mult } \circ\left(F_{\mathcal{R}} \otimes \mathrm{Id}\right)}{\otimes} \mathcal{R}=: \mathrm{HH}_{\bullet}\left(\mathcal{R}, F_{\mathcal{R}}\right),
$$

corresponding to the left copy of $\mathcal{R}$ in the tensor product.

Recall also that $\mathrm{HH}_{\bullet}\left(\mathcal{R}, F_{\mathcal{R}}\right)$ is itself a symmetric monoidal category, so for any $\mathcal{F} \in \mathrm{HH}_{\bullet}\left(\mathcal{R}, F_{\mathcal{R}}\right)$ it makes sense to consider

$$
\iota(r) \otimes \mathcal{F} \in \mathrm{HH}_{\bullet}\left(\mathcal{R}, F_{\mathcal{R}}\right)
$$

4.4.3. We claim:

Theorem 4.4.4. There exists a canonical isomorphism

$$
\operatorname{Tr}\left(H_{r} \circ F_{\mathcal{M}}, \mathcal{M}\right) \simeq \mathcal{H}_{o m_{\mathrm{HH}}\left(\mathcal{R}, F_{\mathcal{R}}\right)}\left(\mathbf{1}_{\mathrm{HH}}\left(\mathcal{R}, F_{\mathcal{R}}\right) \iota(r) \otimes \operatorname{Tr}_{\mathcal{R}}^{\mathrm{enh}}\left(F_{\mathcal{M}}, \mathcal{M}\right)\right),
$$

functorial in $r \in \mathcal{R}$. This isomorphism is compatible with the actions of the two sides of (3.20).

The rest of this subsection is devoted to the proof of this theorem.

4.4.5. For $\mathcal{M}$ as in the theorem, denote by $F_{\mathcal{N}, r}$ the composite $H_{r} \circ F_{\mathcal{X}}$. Note that since $\mathcal{R}$ is symmetric monoidal, $F_{\mathcal{M}, r}$ is also compatible with the action of $F_{\mathcal{R}}$ on $\mathcal{R}$. By Theorem 3.8.5, we have

$$
\operatorname{Tr}\left(H_{r} \circ F_{\mathcal{M}}, \mathcal{M}\right) \simeq \mathcal{H}^{\circ o m_{\mathrm{HH}}\left(\mathcal{R}, F_{\mathcal{R}}\right)}\left(\mathbf{1}_{\mathrm{HH} \bullet\left(\mathcal{R}, F_{\mathcal{R}}\right)}, \operatorname{Tr}_{\mathcal{R}}^{\mathrm{enh}}\left(F_{\mathcal{M}, r}, \mathcal{M}\right)\right)
$$

Hence, in order to prove Theorem 4.4.4, it suffices to establish the following:

Theorem 4.4.6. There exists a canonical isomorphism

$$
\iota(r) \otimes \operatorname{Tr}_{\mathcal{R}}^{\mathrm{enh}}\left(F_{\mathcal{M}}, \mathcal{M}\right) \simeq \operatorname{Tr}_{\mathcal{R}}^{\mathrm{enh}}\left(F_{\mathcal{M}, r}, \mathcal{M}\right)
$$

as objects of $\mathrm{HH}_{\bullet}\left(\mathcal{R}, F_{\mathcal{R}}\right)$.

$\square[$ Theorem 4.4 .4

4.4.7. Proof of Theorem 4.4.6. Consider the diagram

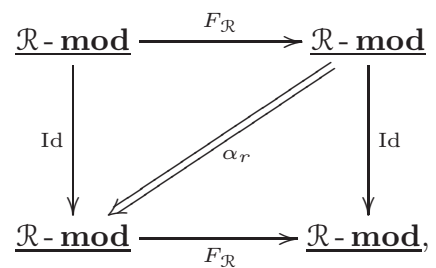

where $\alpha_{r}$ is the 2-morphism, which, when evaluated on $\mathcal{M}^{\prime} \in \mathcal{R}$ - $\bmod$ (see Sect. 3.6.2 for what evaluation means), acts as

$$
H_{r}: \mathcal{M}^{\prime} \rightarrow \mathcal{M}^{\prime},
$$

viewed as a map of $\mathcal{R}$-module categories.

Concatenating with the diagram

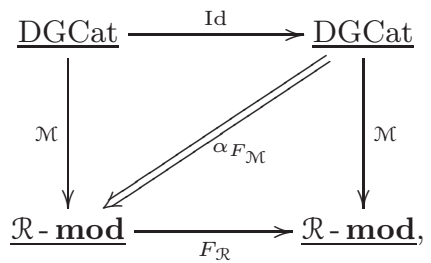


which produces $\operatorname{Tr}_{\mathcal{R}}^{\mathrm{enh}}\left(F_{\mathcal{M}}, \mathcal{M}\right)$, we obtain the diagram

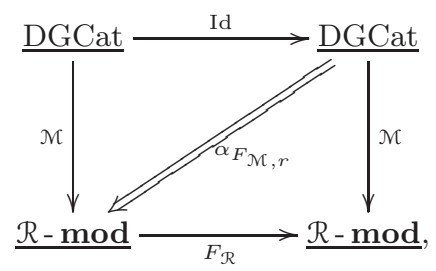

which produces $\operatorname{Tr}_{\mathcal{R}}^{\mathrm{enh}}\left(F_{\mathcal{M}, r}, \mathcal{M}\right)$.

Since the formation of trace is compatible with compositions, it suffices to prove the following:

Proposition 4.4.8. The map

$$
\mathrm{HH}_{\bullet}\left(\mathcal{R}, F_{\mathcal{R}}\right) \rightarrow \mathrm{HH}_{\bullet}\left(\mathcal{R}, F_{\mathcal{R}}\right),
$$

induced by 4.17), is given by $\iota(r) \otimes-$.

$\square[$ Theorem 4.4.6

Proof of Proposition 4.4.8. Consider the corresponding diagram

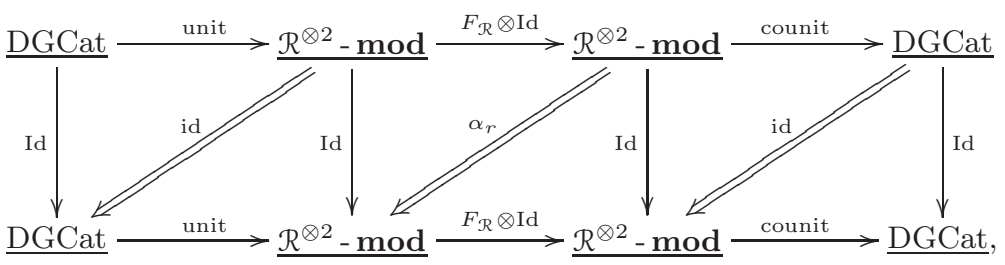

which gives rise to 4.18).

The 2-morphism in the inner square, when evaluated on $\mathcal{Q} \in(\mathcal{R} \otimes \mathcal{R})$ - $\bmod$, acts as

$$
H_{r} \otimes \mathrm{Id} .
$$

This makes the assertion concerning (4.18) manifest.

Remark 4.4.9. The proof of Theorem 4.4.6 can be reformulated as the combination of the following two assertions. Consider the $\mathcal{R}$-module which is $\mathcal{R}$ itself, equipped with the endofunctor $F_{\mathcal{R}, r}:=H_{r} \circ F_{\mathcal{R}}$.

The first assertion is that there is a canonical isomorphism in $\mathrm{HH}_{\bullet}\left(\mathcal{R}, F_{\mathcal{R}}\right)$

$$
\operatorname{Tr}_{\mathcal{R}}^{\mathrm{enh}}\left(F_{\mathcal{R}, r}, \mathcal{R}\right) \simeq \iota(r) .
$$

This is a particular case of Proposition 4.4.8

For the second assertion, consider $\left(\underline{\mathcal{R}-\mathbf{m o d}}, \operatorname{Res}_{F_{\mathcal{R}}}\right)$ as a commutative algebra object in the symmetric monoidal category $L(\text { Morita(DGCat) })_{\text {rgd }}$, and the monoid

$$
\operatorname{Maps}_{L(\text { Morita }(\text { DGCat }))_{\text {rgd }}}\left((\underline{\text { DGCat }}, I d),\left(\underline{\mathcal{R}-\mathbf{m o d}}, \operatorname{Res}_{F_{\mathcal{R}}}\right)\right) \text {, }
$$

where we recall that $(\underline{\text { DGCat }}, I d)$ is the unit in $L(\text { Morita(DGCat) })_{\text {rgd }}$.

The second assertion is that $\operatorname{Tr}_{\mathcal{R}}^{\text {enh }}$ is a symmetric monoidal functor

$$
\operatorname{Maps}_{L(\operatorname{Morita}(\text { DGCat }))_{\text {rgd }}}\left((\underline{\text { DGCat }}, I d),\left(\underline{\mathcal{R}-\bmod }, \operatorname{Res}_{F_{\mathcal{R}}}\right)\right) \rightarrow \mathrm{HH}_{\bullet}\left(\mathcal{R}, F_{\mathcal{R}}\right) .
$$

This follows from the fact that the functor Tr of (3.10) is symmetric monoidal.

To deduce Theorem 4.4.6 we note that for any $\left(\mathcal{M}, F_{\mathcal{M}}\right)$ we have

$$
\left(\mathcal{M}, F_{\mathcal{M}, r}\right) \simeq\left(\mathcal{R}, F_{\mathcal{R}, r}\right) \otimes\left(\mathcal{M}, F_{\mathcal{M}}\right)
$$

as objects in 4.19). 
Note also that for this manipulation, we regarded $L$ (Morita(DGCat) $)_{\text {rgd }}$ as an $(\infty, 1)$-category (i.e., we did not need to consider non-invertible 3-morphisms, as in Sect. 3.9). More generally, $\operatorname{Tr}_{\mathcal{R}}^{\text {enh }}$ can be considered as a functor of symmetric monoidal categories

$$
\operatorname{Maps}_{L(\text { Morita(DGCat }))_{\text {rgd }}}\left((\underline{\text { DGCat }}, \mathrm{Id}),\left(\underline{\mathcal{R}-\mathbf{m o d}}, \operatorname{Res}_{F_{\mathcal{R}}}\right)\right) \rightarrow \mathrm{HH}_{\bullet}\left(\mathcal{R}, F_{\mathcal{R}}\right),
$$

whose source is the symmetric monoidal category

$$
\operatorname{Maps}_{L(\operatorname{Morita}(\text { DGCat }))_{\text {rgd }}}\left((\underline{\text { DGCat }}, I d),\left(\underline{\mathcal{R}-\mathbf{m o d}}, \operatorname{Res}_{F_{\mathcal{R}}}\right)\right),
$$

where $\operatorname{Maps}(-,-)$ denotes the $(\infty, 1)$-category of maps between objects in a given $(\infty, 2)$-category.

4.5. Cyclicity and observables. We return to the setting of Theorem 4.4.4. Let us now fix two objects $r_{1}, r_{2} \in \mathcal{R}$.

4.5.1. On the one hand, consider the following two modules over $\operatorname{Tr}\left(F_{\mathcal{R}}, \mathcal{R}\right)$ :

$$
\operatorname{Tr}\left(H_{r_{1}} \circ H_{r_{2}} \circ F_{\mathcal{M}}, \mathcal{M}\right) \text { and } \operatorname{Tr}\left(H_{F_{\mathcal{R}}\left(r_{1}\right)} \circ H_{r_{2}} \circ F_{\mathcal{M}}, \mathcal{M}\right) \text {. }
$$

We claim that there exists a canonical isomorphism

$$
\operatorname{Tr}\left(H_{r_{1}} \circ H_{r_{2}} \circ F_{\mathcal{M}}, \mathcal{M}\right) \simeq \operatorname{Tr}\left(H_{F_{\mathcal{R}}\left(r_{1}\right)} \circ H_{r_{2}} \circ F_{\mathcal{M}}, \mathcal{M}\right)
$$

Indeed, it is obtained as the composition

$$
\begin{array}{ccc}
\operatorname{Tr}\left(H_{r_{1}} \circ H_{r_{2}} \circ F_{\mathcal{M}}, \mathcal{M}\right) \quad \stackrel{\text { cyclicity of trace }}{\sim} \operatorname{Tr}\left(H_{r_{2}} \circ F_{\mathcal{M}} \circ H_{r_{1}}, \mathcal{M}\right) & \downarrow \sim \\
\operatorname{Tr}\left(H_{F_{\mathcal{R}}\left(r_{1}\right)} \circ H_{r_{2}} \circ F_{\mathcal{M}}, \mathcal{M}\right) & \longleftarrow \sim & \operatorname{Tr}\left(H_{r_{2}} \circ H_{F_{\mathcal{R}}\left(r_{1}\right)} \circ F_{\mathcal{M}}, \mathcal{M}\right),
\end{array}
$$

where the second arrow uses the isomorphism

$$
F_{\mathcal{M}} \circ H_{r_{1}} \simeq H_{F_{\mathcal{R}}\left(r_{1}\right)} \circ F_{\mathcal{M}}
$$

and the last arrow uses the fact that the product on $\mathcal{R}$ is commutative.

4.5.2. On the other hand, consider the following two modules over $\mathcal{E} n d_{\mathrm{HH}\left(\mathcal{R}, F_{\mathcal{R}}\right)}\left(\mathbf{1}_{\mathrm{HH}_{\bullet}\left(\mathcal{R}, F_{\mathcal{R}}\right)}\right)$ :

$$
\mathcal{H}_{o m_{\mathrm{HH}}\left(\mathcal{R}, F_{\mathcal{R}}\right)}\left(\mathbf{1}_{\mathrm{HH} \cdot\left(\mathcal{R}, F_{\mathcal{R}}\right)}, \iota\left(r_{1} \otimes r_{2}\right) \otimes \operatorname{Tr}_{\mathcal{R}}^{\mathrm{enh}}\left(F_{\mathcal{M}}, \mathcal{M}\right)\right)
$$

and

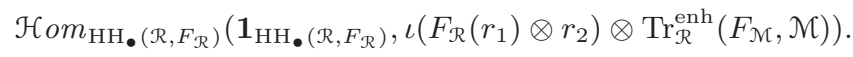

We claim that there is a canonical isomorphism

$$
\begin{aligned}
& \mathcal{H}_{o m_{\mathrm{HH}}\left(\mathcal{R}, F_{\mathcal{R}}\right)}\left(\mathbf{1}_{\mathrm{HH}_{\bullet}\left(\mathcal{R}, F_{\mathcal{R}}\right)}, \iota\left(r_{1} \otimes r_{2}\right) \otimes \operatorname{Tr}_{\mathcal{R}}^{\mathrm{enh}}\left(F_{\mathcal{M}}, \mathcal{M}\right)\right)
\end{aligned}
$$

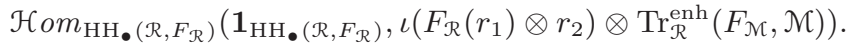

Indeed, this follows from the fact that

$$
\iota(r) \simeq \iota\left(F_{\mathcal{R}}(r)\right) .
$$

4.5.3. We now claim:

Lemma 4.5.4. The isomorphisms (4.23) and (4.26) match up under the isomorphisms

$$
\operatorname{Tr}\left(H_{r_{1}} \circ H_{r_{2}} \circ F_{\mathcal{M}}, \mathcal{M}\right) \simeq \mathcal{H}^{\circ o m_{\mathrm{HH}}\left(\mathcal{R}, F_{\mathcal{R}}\right)}\left(\mathbf{1}_{\mathrm{HH}}\left(\mathcal{R}, F_{\mathcal{R}}\right), \iota\left(r_{1} \otimes r_{2}\right) \otimes \operatorname{Tr}_{\mathcal{R}}^{\mathrm{enh}}\left(F_{\mathcal{M}}, \mathcal{M}\right)\right)
$$

and

$$
\operatorname{Tr}\left(H_{F_{\mathcal{R}}\left(r_{1}\right)} \circ H_{r_{2}} \circ F_{\mathcal{M}}, \mathcal{M}\right) \simeq \mathcal{H} \mathcal{H}_{\mathrm{HH}_{\bullet}\left(\mathcal{R}, F_{\mathcal{R}}\right)}\left(\mathbf{1}_{\mathrm{HH}}\left(\mathcal{R}, F_{\mathcal{R}}\right), \iota\left(F_{\mathcal{R}}\left(r_{1}\right) \otimes r_{2}\right) \otimes \operatorname{Tr}_{\mathcal{R}}^{\mathrm{enh}}\left(F_{\mathcal{M}}, \mathcal{M}\right)\right)
$$

of Theorem 4.4 .4$. 
Proof. Note first that it suffices to show the assertion in the particular case $r_{2}=\mathbf{1}_{\mathcal{R}}$ and $r_{1}=r$. (Namely, the general case would follow from this particular case by replacing $F_{\mathcal{M}}$ by $F_{\mathcal{M}, r_{2}}$ ).

Thus, we have to show that under the isomorphisms

$$
\operatorname{Tr}\left(H_{r} \circ F_{\mathcal{M}}, \mathcal{M}\right) \simeq \mathcal{H}^{\circ o m_{\mathrm{HH}}\left(\mathcal{R}, F_{\mathcal{R}}\right)}\left(\mathbf{1}_{\mathrm{HH}\left(\mathcal{R}, F_{\mathcal{R}}\right)}, \iota(r) \otimes \operatorname{Tr}_{\mathcal{R}}^{\mathrm{enh}}\left(F_{\mathcal{M}}, \mathcal{M}\right)\right)
$$

and

$$
\operatorname{Tr}\left(H_{F_{\mathcal{R}}(r)} \circ F_{\mathcal{M}}, \mathcal{M}\right) \simeq \mathcal{H} \operatorname{com}_{\mathrm{HH}_{\bullet}\left(\mathcal{R}, F_{\mathcal{R}}\right)}\left(\mathbf{1}_{\mathrm{HH}_{\bullet}\left(\mathcal{R}, F_{\mathcal{R}}\right)}, \iota\left(F_{\mathcal{R}}(r)\right) \otimes \operatorname{Tr}_{\mathcal{R}}^{\mathrm{enh}}\left(F_{\mathcal{M}}, \mathcal{M}\right)\right)
$$

of Theorem 4.4.4 the composition

$$
\operatorname{Tr}\left(H_{r} \circ F_{\mathcal{M}}, \mathcal{M}\right) \stackrel{\text { cyclicity of trace }}{\longrightarrow} \operatorname{Tr}\left(F_{\mathcal{M}} \circ H_{r}, \mathcal{M}\right) \stackrel{4.25}{\sim} \operatorname{Tr}\left(H_{F_{\mathcal{R}}(r)} \circ F_{\mathcal{M}}, \mathcal{M}\right),
$$

of the left hand sides corresponds to the isomorphism induced by (4.27) of the right hand side.

Note that isomorphism (3.5), corresponding to the pair of diagrams
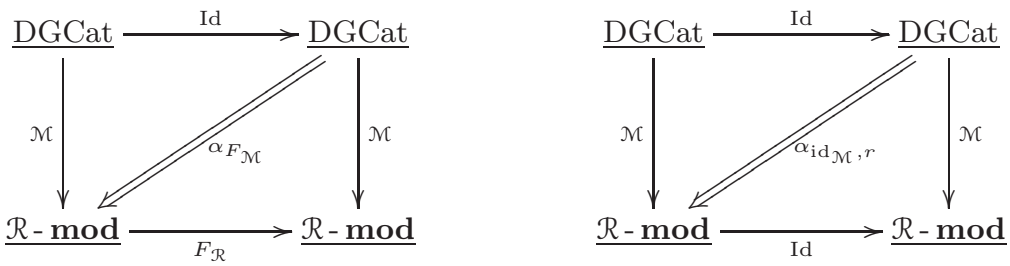

induces an isomorphism

$$
\operatorname{Tr}_{\mathcal{R}}^{\mathrm{enh}}\left(H_{r} \circ F_{\mathcal{M}}, \mathcal{M}\right) \simeq \operatorname{Tr}_{\mathcal{R}}^{\mathrm{enh}}\left(F_{\mathcal{M}} \circ H_{r}, \mathcal{M}\right)
$$

of objects of $\mathrm{HH}_{\bullet}\left(\mathcal{R}, F_{\mathcal{R}}\right)$.

To finish the proof, it suffices to show that diagrams

$$
\begin{aligned}
& \operatorname{Tr}\left(H_{r} \circ F_{\mathcal{M}}, \mathcal{M}\right) \stackrel{3.21}{\sim} \mathcal{H}_{o} m_{\mathrm{HH}_{\bullet}\left(\mathcal{R}, F_{\mathcal{R}}\right)}\left(\mathbf{1}_{\mathrm{HH}}\left(\mathcal{R}, F_{\mathcal{R}}\right), \operatorname{Tr}_{\mathcal{R}}^{\mathrm{enh}}\left(H_{r} \circ F_{\mathcal{M}}, \mathcal{M}\right)\right) \\
& \sim \text { cyclicity of trace } \quad \sim \downarrow \sqrt{4.30} \\
& \operatorname{Tr}\left(F_{\mathcal{M}} \circ H_{r}, \mathcal{M}\right) \stackrel{3.21}{\sim} \mathcal{H} \mathcal{H}_{\mathrm{HH}_{\bullet}\left(\mathcal{R}, F_{\mathcal{R}}\right)}\left(\mathbf{1}_{\mathrm{HH}_{\bullet}\left(\mathcal{R}, F_{\mathcal{R}}\right)}, \operatorname{Tr}_{\mathcal{R}}^{\mathrm{enh}}\left(F_{\mathcal{M}} \circ H_{r}, \mathcal{M}\right)\right)
\end{aligned}
$$

and

$$
\begin{array}{ccc}
\operatorname{Tr}_{\mathcal{R}}^{\mathrm{enh}}\left(H_{r} \circ F_{\mathcal{M}}, \mathcal{M}\right) & \stackrel{4.16}{\longrightarrow} & \left.\iota(r) \otimes \operatorname{Tr}_{\mathcal{R}}^{\mathrm{enh}}\left(F_{\mathcal{M}}, \mathcal{M}\right)\right) \\
& \sim \downarrow 4.25 \\
\operatorname{Tr}_{\mathcal{R}}^{\mathrm{enh}}\left(H_{F_{\mathcal{R}}(r)} \circ F_{\mathcal{M}}, \mathcal{M}\right) & \left.\stackrel{4.30}{\stackrel{\sim 4.16}{\longrightarrow}} \iota\left(F_{\mathcal{R}}(r)\right) \otimes \operatorname{Tr}_{\mathcal{R}}^{\mathrm{enh}}\left(F_{\mathcal{M}}, \mathcal{M}\right)\right)
\end{array}
$$

are commutative.

We will deduce both assertions from the commutativity of the diagram (3.9).

To show the commutativity of (4.31), consider the following pair of diagrams:
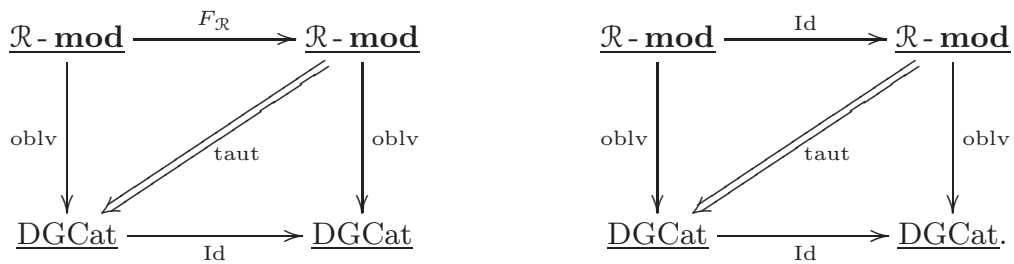

Arguing as in Sect. 3.10.10 or Sect. 3.12.2 the isomorphism (3.5) corresponding to the diagrams in (4.33) can be identified with the identity endomorphism on $\mathcal{H}_{0} m_{\mathrm{HH}}\left(\mathcal{R}, F_{\mathcal{R}}\right)\left(\mathbf{1}_{\mathrm{HH}_{\bullet}\left(\mathcal{R}, F_{\mathcal{R}}\right)},-\right)$. 
Since the vertical compositions of the diagrams appearing in 44.29) and (4.33) are isomorphic, respectively, to
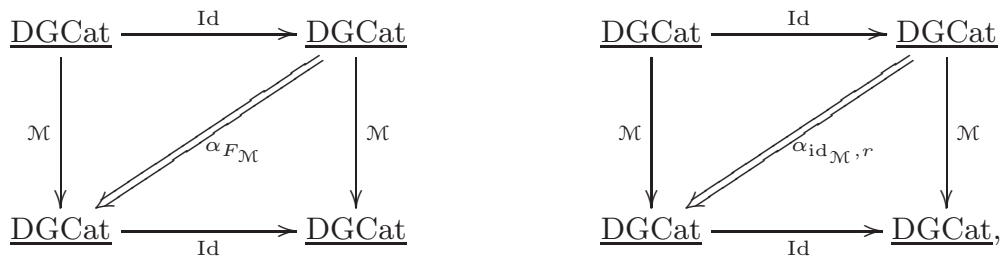

the commutative diagram (3.9), corresponding to (4.29) and (4.33), naturally identifies with (4.31).

To show the commutativity of (4.32), note that the diagrams in (4.29) can be written as vertical compositions of the diagrams
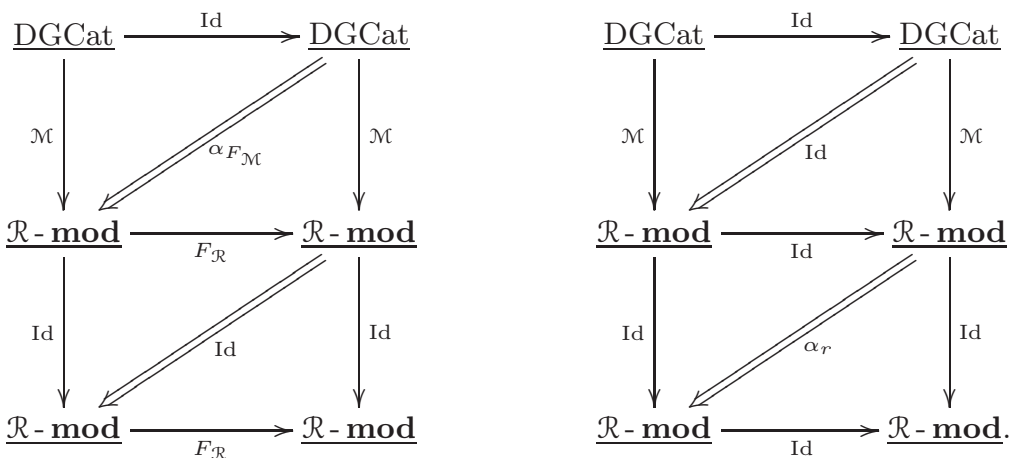

Arguing as in the proof of Proposition 4.4.8 the isomorphism (3.5) corresponding to the bottom part of (4.35) can be identified with the isomorphism of functors

$$
\iota(r) \otimes-\simeq \iota\left(F_{\mathcal{R}}(r)\right) \otimes-,
$$

induced by isomorphism 4.27.

Since the isomorphism (3.5) corresponding to the top part of 4.35) can be identified with the identity endomorphism of $\operatorname{Tr}_{\mathcal{R}}^{\text {enh }}\left(F_{\mathcal{M}}, \mathcal{M}\right)$, the commutative diagram (3.9), corresponding to the diagrams in (4.35), naturally identifies with (4.32).

\section{The "ShtukA" COnstruction}

In this section we combine all the ingredients developed in the previous sections to obtain our the toy model for the shtuka construction.

\subsection{The universal shtuka.}

5.1.1. Let $\mathcal{A}$ be a rigid symmetric monoidal category and $Y$ an object of Spc. Consider the symmetric monoidal category $\mathcal{A}^{\otimes Y}$.

Let $\phi$ be an endomorphism of $Y$, and let $\mathcal{A}^{\otimes \phi}$ be the induced (symmetric monoidal) endofunctor of $\mathcal{A}^{\otimes Y}$.

Let $\mathcal{M}$ be a dualizable DG category, equipped with an action of $\mathcal{A}^{\otimes Y}$. Let $F_{\mathcal{M}}$ be an endofunctor of $\mathcal{M}$ compatible with the action of $\mathcal{A}^{\otimes \phi}$ on $\mathcal{A}^{\otimes Y}$. 
5.1.2. According to Sect. 3.8 .2 to this data we can attach an object

$$
\operatorname{Tr}_{\mathcal{A}}^{\mathrm{enh}}\left(F_{\mathcal{X}}, \mathcal{M}\right) \in \mathrm{HH}_{\bullet}\left(\mathcal{A}^{\otimes Y}, \mathcal{A}^{\otimes \phi}\right) .
$$

We identify

by Proposition 3.7 .5

$$
\mathrm{HH}_{\bullet}\left(\mathcal{A}^{\otimes Y}, \mathcal{A}^{\otimes \phi}\right) \simeq \mathcal{A}^{\otimes Y / \phi}
$$

We will use yet another notation for $\operatorname{Tr}_{\mathcal{A} \otimes Y}^{\text {enh }}\left(F_{\mathcal{M}}, \mathcal{M}\right)$, namely

$$
\operatorname{Sht}_{\mathcal{M}, F_{\mathcal{M}}, \text { univ }} \in \mathcal{A}^{\otimes Y / \phi} \text {, }
$$

and call it "the universal shtuka".

5.1.3. According to Theorem 3.8.5 we have an identification

$$
\mathcal{E} n d_{\mathcal{A} \otimes Y / \phi}\left(\mathbf{1}_{\mathcal{A} \otimes Y / \phi}\right) \simeq \operatorname{Tr}\left(\mathcal{A}^{\otimes \phi}, \mathcal{A}^{\otimes Y}\right)
$$

and

$$
\mathcal{H}_{\mathcal{A}} \mathrm{m}_{\mathcal{A} \otimes Y / \phi}\left(\mathbf{1}_{\mathcal{A} \otimes Y / \phi}, \operatorname{Sht}_{\mathcal{M}, F_{\mathcal{M}}, \text { univ }}\right) \simeq \operatorname{Tr}\left(F_{\mathcal{M}}, \mathcal{M}\right) .
$$

The isomorphisms (5.1) and (5.2) are compatible with the action of $\mathcal{E} n d_{\mathcal{A}^{\otimes Y / \phi}}\left(\mathbf{1}_{\mathcal{A} \otimes Y / \phi}\right)$ on $\mathcal{H}_{o m_{\mathcal{A}} \otimes Y / \phi}\left(\mathbf{1}_{\mathcal{A} \otimes Y / \phi}, \operatorname{Sht}_{\mathcal{M}, F_{\mathcal{M}} \text {, univ }}\right)$ and the action of $\operatorname{Tr}\left(\mathcal{A}^{\otimes \phi}, \mathcal{A}^{\otimes Y}\right)$ on $\operatorname{Tr}\left(F_{\mathcal{M}}, \mathcal{M}\right)$.

In particular, $\operatorname{Tr}\left(F_{\mathcal{M}}, \mathcal{M}\right)$ carries an action of $\mathcal{E} n d_{\mathcal{A} \otimes Y / \phi}\left(\mathbf{1}_{\mathcal{A} \otimes Y / \phi}\right)$.

5.1.4. Example. Let e be a field of characteristic zero. Let $\mathcal{A}=\operatorname{Rep}(\mathrm{G})$ and assume that $Y$ has finitely many connected components. Then according to Theorem 1.5.5.

$$
\left.\mathcal{A}^{\otimes Y / \phi} \simeq \mathrm{QCoh} \operatorname{LocSys}_{\mathrm{G}}(Y / \phi)\right),
$$

and hence

$$
\mathcal{E} n d_{\mathcal{A} \otimes Y / \phi}\left(\mathbf{1}_{\mathcal{A} \otimes Y / \phi}\right) \simeq \Gamma\left(\operatorname{LocSys}_{\mathrm{G}}(Y / \phi), \mathcal{O}_{\operatorname{LocSys}_{\mathrm{G}}(Y / \phi)}\right)
$$

We obtain that in this case

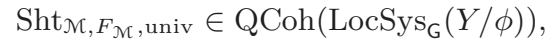

and the vector space

$$
\operatorname{Tr}\left(F_{\mathcal{M}}, \mathcal{M}\right) \simeq \Gamma\left(\operatorname{LocSys}_{G}(Y / \phi), \operatorname{Sht}_{\mathcal{M}}, F_{\mathcal{M}}, \text { univ }\right)
$$

carries an action of the algebra

$$
\Gamma\left(\operatorname{LocSys}_{\mathrm{G}}(Y / \phi), \mathcal{O}_{\operatorname{LocSys}_{\mathrm{G}}(Y / \phi)}\right) .
$$

5.1.5. According to Sect. 1.8.5 we can think of an object of $\mathcal{A}^{\otimes Y / \phi}$ as a compatible family of functors

$$
\mathcal{A}^{\otimes I} \rightarrow \operatorname{LS}\left((Y / \phi)^{I}\right), \quad I \in \text { fSet } .
$$

Applying this to the object

$$
\operatorname{Sht}_{\mathcal{M}, F_{\mathcal{M}}, \text { univ }} \in \mathcal{A}^{\otimes Y / \phi}
$$

we obtain a family of functors denoted

$$
\operatorname{Sht}_{\mathcal{M}, F_{\mathcal{M}}, Y / \phi, I}: \mathcal{A}^{\otimes I} \rightarrow \operatorname{LS}\left((Y / \phi)^{I}\right) .
$$

We will denote by $\operatorname{Sht}_{\mathcal{M}, F_{\mathcal{M}}, Y, I}$ the composite of $\operatorname{Sht}_{\mathcal{M}, F_{\mathcal{M}}, Y / \phi, I}$ with the pullback functor

$$
\operatorname{LS}\left((Y / \phi)^{I}\right) \rightarrow \operatorname{LS}\left(Y^{I}\right)
$$

corresponding to the projection $Y \rightarrow Y / \phi$.

By Sect. 1.8.6, the object

can be canonically identified with

$$
\operatorname{Sht}_{\mathcal{M}, F_{\mathcal{M}}, Y / \phi, \emptyset} \in \text { Vect }
$$

$$
\mathcal{H}_{\text {oom }}{ }_{\mathcal{A} \otimes Y / \phi}\left(\mathbf{1}_{\mathcal{A} \otimes Y / \phi}, \operatorname{Sht}_{\mathcal{M}, F_{\mathcal{M}}, \text { univ }}\right) \simeq \operatorname{Tr}\left(F_{\mathcal{M}}, \mathcal{M}\right) .
$$


5.1.6. The goals of the present section are the following:

-Describe the functors $\operatorname{Sht}_{\mathcal{M}, F_{\mathcal{M}}, Y, I}$ explicitly via the usual (i.e., 1-categorical) trace construction;

-Describe the descent of Sht $\operatorname{S}_{\mathcal{M}, F_{\mathcal{M}}, Y, I}$ to $\mathrm{Sht}_{\mathcal{M}, F_{\mathcal{M}}, Y / \phi, I}$ via the action of "partial Frobeniuses";

-Describe the action of $\mathcal{E} n d_{\mathcal{A} \otimes Y / \phi}\left(\mathbf{1}_{\mathcal{A} \otimes Y / \phi}\right)$ on $\operatorname{Tr}\left(F_{\mathcal{M}}, \mathcal{M}\right)$ in terms of the functors $\operatorname{Sht}_{\mathcal{M}, F_{\mathcal{M}}, Y / \phi, I}$ via the "excursion operators";

-Prove the "S=T" identity (see Sect. 5.5 for what this means).

All of the above will amount to an application of the constructions of the previous subsections.

5.1.7. Before we proceed further, let us note the following functoriality property of the shtuka construction in $Y$ :

Let us be given another space $Y^{\prime}$, equipped with an endomorphism $\phi^{\prime}$, and a map of spaces $\psi$ : $Y^{\prime} \rightarrow Y$ that intertwines $\phi^{\prime}$ and $\phi$. Let $\operatorname{Res}_{\psi}(\mathcal{M})$ denote the $\mathcal{A}^{\otimes Y^{\prime}}$-module category, obtained from $\mathcal{M}$ by restricting along $\psi$.

Consider the resulting objects

$$
\operatorname{Sht}_{\mathcal{M}, F_{\mathcal{X}}, \text { univ }} \in \mathcal{A}^{\otimes Y / \phi} \text { and } \operatorname{Sht}_{\operatorname{Res}_{\psi}(\mathcal{M}), F_{\mathcal{M}}, \text { univ }} \in \mathcal{A}^{\otimes Y^{\prime} / \phi^{\prime}} .
$$

From 3.35 and we obtain:

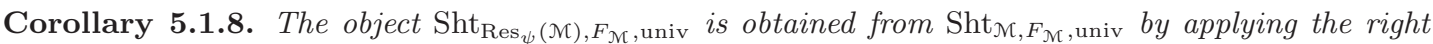
adjoint of the functor

$$
\mathcal{A}^{\otimes \psi}: \mathcal{A}^{\otimes Y^{\prime} / \phi^{\prime}} \rightarrow \mathcal{A}^{\otimes Y / \phi} .
$$

Remark 5.1.9. Note that the equivalences

$$
\mathcal{A}^{\otimes Y / \phi} \simeq\left(\mathcal{A}^{\otimes Y / \phi}\right)^{\vee} \text { and } \mathcal{A}^{\otimes Y^{\prime} / \phi^{\prime}} \simeq\left(\mathcal{A}^{\otimes Y^{\prime} / \phi^{\prime}}\right)^{\vee}
$$

from Sect. 1.8.5 identify the right adjoint $\left(\mathcal{A}^{\otimes \psi}\right)^{R}$ of $\mathcal{A}^{\otimes \psi}$ with the dual $\left(\mathcal{A}^{\otimes \psi}\right)^{\vee}$ (see [GR1, Section 1, Lemma 9.2.6]).

Therefore it follows from Corollary 5.1.8 that the functor

$$
\mathcal{S}_{Y^{\prime} / \phi^{\prime}}: \mathcal{A}^{\otimes Y^{\prime} / \phi^{\prime}} \rightarrow \text { Vect }
$$

corresponding to $\operatorname{Sht}_{\operatorname{Res}_{\psi}(\mathcal{M}), F_{\mathcal{M}} \text {, univ }} \in \mathcal{A}^{\otimes Y^{\prime} / \phi^{\prime}}$, is obtained from the functor

$$
\mathcal{S}_{Y / \phi}: \mathcal{A}^{\otimes Y / \phi} \rightarrow \text { Vect }
$$

corresponding to $\operatorname{Sht}_{\mathcal{M}, F_{\mathcal{M}} \text {, univ }} \in \mathcal{A}^{\otimes Y / \phi}$, by precomposition with $\mathcal{A}^{\otimes \psi}$.

Remark 5.1.10. Note that in the example of Sect. 5.1.4 the map $\psi$ induces a map

$$
\operatorname{LocSys}_{\mathrm{G}}(\psi): \operatorname{LocSys}_{\mathrm{G}}(Y / \phi) \rightarrow \operatorname{LocSys}_{\mathrm{G}}\left(Y^{\prime} / \phi^{\prime}\right),
$$

and the functors $\mathcal{A}^{\otimes \psi}$ and $\left(\mathcal{A}^{\otimes \psi}\right)^{R}$ identify with $\operatorname{LocSys}_{\mathrm{G}}(\psi)^{*}$ and $\operatorname{LocSys}_{\mathrm{G}}(\psi)_{*}$, respectively.

5.2. Explicit description of $I$-legged shtukas. We retain the setting of Sect. 5.1.1.

5.2.1. Fix a finite set $I$. The evaluation map

$$
Y^{I} \times I \rightarrow Y
$$

defines a map from $Y^{I}$ to the space of symmetric monoidal functors $\mathcal{A}^{\otimes I} \rightarrow \mathcal{A}^{\otimes Y}$, and hence to the space of actions of $\mathcal{A}^{\otimes I}$ on $\mathcal{M}$.

Define the functor

as follows:

$$
\operatorname{Sht}_{\mathcal{M}, F_{\mathcal{M}}, Y, I}^{\prime}: Y^{I} \times \mathcal{A}^{\otimes I} \rightarrow \text { Vect }
$$

It sends

$$
\underline{y} \in Y^{I}, r \in \mathcal{A}^{\otimes I} \mapsto \operatorname{Tr}\left(H_{r_{\underline{y}}} \circ F_{\mathcal{M}}, \mathcal{M}\right),
$$


where $r_{y}$ denotes the image of $r$ along the functor

$$
\mathcal{A}^{\otimes I} \stackrel{\underline{y}}{\rightarrow} \mathcal{A}^{\otimes Y} .
$$

See Sect. 3.5.11 for an explanation of how this relates to the usual notion of shtukas.

Remark 5.2.2. We can tautologically rewrite the functor

$$
Y^{I} \times \mathcal{A}^{\otimes I} \rightarrow \operatorname{End}(\mathcal{M}), \quad(\underline{y}, r) \mapsto H_{r_{\underline{y}}},
$$

which appears in the definition of $\operatorname{Sht}_{\mathcal{M}, F_{\mathcal{M}}, Y, I}^{\prime}$, as follows:

Recall that according to Sect. 1.7.3, a datum of action of $\mathcal{A}^{\otimes Y}$ on $\mathcal{M}$ gives rise to a map

$$
\mathcal{A}^{\otimes I} \rightarrow \operatorname{End}(\mathcal{M}) \otimes \operatorname{LS}\left(Y^{I}\right)
$$

or, equivalently by Proposition 1.4.10 to a map

$$
Y^{I} \rightarrow \operatorname{Maps}\left(\mathcal{A}^{\otimes I}, \operatorname{End}(\mathcal{M})\right) .
$$

The map (5.3) is the one corresponding to (5.4).

5.2.3. We claim:

Proposition 5.2.4. The functor

$$
\mathcal{A}^{\otimes I} \rightarrow \operatorname{LS}\left(Y^{I}\right)
$$

corresponding to $\operatorname{Sht}_{\mathcal{M}, F_{\mathcal{M}}, Y, I}^{\prime}$, identifies canonically with $\operatorname{Sht}_{\mathcal{M}, F_{\mathcal{M}}, Y, I}$.

Proof. Taking into account Sect. 1.8.5 we have to establish the isomorphism

$$
\mathcal{H}^{\circ o m_{\mathcal{A} \otimes Y / \phi}}\left(\mathbf{1}_{\mathcal{A} \otimes Y / \phi}, r_{\underline{\bar{y}}} \otimes \operatorname{Sht}_{\mathcal{M}, F_{\mathcal{M}}, \text { univ }}\right) \simeq \operatorname{Tr}\left(H_{r_{\underline{y}}} \circ F_{\mathcal{M}}, \mathcal{M}\right),
$$

functorial in $\underline{y} \in Y^{I}$, where $\underline{\bar{y}}$ is the image of $\underline{y}$ under the projection

$$
Y \rightarrow Y / \phi \text {. }
$$

Now the assertion follows from Theorem 4.4.4 using the fact that

$$
r_{\underline{\bar{y}}} \simeq \iota\left(r_{\underline{y}}\right) \text {. }
$$

\subsection{Partial Frobeniuses.}

5.3.1. Since the object

is the image of the object

$$
\operatorname{Sht}_{\mathcal{M}, F_{\mathcal{M}}, Y, I} \in \operatorname{Funct}\left(\mathcal{A}^{\otimes I}, \operatorname{LS}\left(Y^{I}\right)\right)
$$

$$
\operatorname{Sht}_{\mathcal{M}, F_{\mathcal{M}}, Y / \phi, I} \in \operatorname{Funct}\left(\mathcal{A}^{\otimes I}, \operatorname{LS}\left((Y / \phi)^{I}\right)\right)
$$

under the pullback functor

$$
\mathrm{LS}\left((Y / \phi)^{I}\right) \rightarrow \operatorname{LS}\left(Y^{I}\right)
$$

the former should carry the structure of equivariance with respect to the endomorphisms that act as $\phi$ along each of the $Y$ factors in $Y^{I}$.

Fix an element $i^{1} \in I$ and write $I=\left\{i^{1}\right\} \sqcup I^{\prime}$. We will now write down explicitly the structure of equivariance corresponding to this endomorphism of $Y^{I}$, to be denoted $\phi_{i^{1}}$.

Using the identification of Proposition 5.2.4 this amounts to describing the isomorphism

$$
\operatorname{Sht}_{\mathcal{M}, F_{\mathcal{M}}, Y, I}^{\prime} \circ\left(\phi_{i^{1}} \times \operatorname{Id}_{\mathcal{A} \otimes I}\right) \simeq \operatorname{Sht}_{\mathcal{M}, F_{\mathcal{M}}, Y, I}^{\prime}
$$

as functors

$$
Y^{I} \times \mathcal{A}^{\otimes I} \rightarrow \text { Vect }
$$


5.3.2. By definition, the datum of (5.5) amounts to a system of isomorphisms

$$
\operatorname{Tr}\left(H_{r_{\phi_{i}(\underline{y})}} \circ F_{\mathcal{M}}, \mathcal{M}\right) \simeq \operatorname{Tr}\left(H_{r_{\underline{y}}} \circ F_{\mathcal{M}}, \mathcal{M}\right), \quad r \in \mathcal{A}^{\otimes I} .
$$

Write $\underline{y}$ as

$$
\underline{y}=y^{1} \sqcup \underline{y^{\prime}}, \quad y^{1} \in Y, \underline{y^{\prime}} \in Y^{I^{\prime}} .
$$

It is enough to establish (5.6) for $r$ of the form

$$
r^{1} \otimes r^{\prime}, \quad r^{1} \in \mathcal{A}, r^{\prime} \in \mathcal{A}^{\otimes I^{\prime}} .
$$

Proposition 5.3.3. The map (5.6) is given by the composition

$$
\begin{aligned}
& \operatorname{Tr}\left(H_{r_{y^{1}}} \circ H_{r_{\underline{y^{\prime}}}} \circ F_{\mathcal{M}}, \mathcal{M}\right) \longleftarrow \sim \operatorname{Tr}\left(H_{r_{\underline{y}}} \circ F_{\mathcal{M}}, \mathcal{M}\right) \\
& \sim \text { cyclicity of trace } \\
& \operatorname{Tr}\left(H_{\underline{r^{\prime}}} \circ F_{\mathcal{M}} \circ H_{r_{y^{1}}^{1}}, \mathcal{M}\right) \longrightarrow \operatorname{Tr}\left(H_{\underline{r^{\prime}}} \circ H_{r_{\phi\left(y^{1}\right)}^{1}} \circ F_{\mathcal{M}}, \mathcal{M}\right) \\
& \downarrow \sim \\
& \operatorname{Tr}\left(H_{r_{\phi_{i}(\underline{y})}} \circ F_{\mathcal{M}}, \mathcal{M}\right) \longleftarrow \sim \sim \sim T\left(H_{r_{\phi\left(y^{1}\right)}^{1}} \circ H_{{\underline{r^{\prime}}}^{\prime}} \circ F_{\mathcal{M}}, \mathcal{M}\right) .
\end{aligned}
$$

The proof follows from Lemma 4.5.4

\subsection{Description of the action via excursions.}

5.4.1. Choose a pair of points $\bar{y}_{1}, \bar{y}_{2}$ in $Y / \phi$. Fix finite set $J$ and a $J$-tuple $\gamma^{J}$ of paths $\gamma^{i}$ from $\bar{y}_{1}$ to $\bar{y}_{2}$. Choose an element

$$
\xi \in \mathcal{H} \operatorname{com}\left(\mathbf{1}_{\mathcal{A}}, \text { mult }_{J} \circ \operatorname{mult}_{J}^{R}\left(\mathbf{1}_{\mathcal{A}}\right)\right) .
$$

Let $\operatorname{Exc}_{\text {univ }}\left(\gamma^{J}, \xi\right)$ denote the resulting endomorphism of $\mathbf{1}_{\mathcal{A} \otimes Y / \phi}$, see Sect. 2.8.4

5.4.2. The next assertion follows from the definition of the excursion operators in Sect. 2.8.3

Proposition 5.4.3. The action of $\operatorname{Exc}_{\text {univ }}\left(\gamma^{J}, \xi\right)$ on

$$
\operatorname{Tr}\left(F_{\mathcal{M}}, \mathcal{M}\right) \simeq \mathcal{H}_{\mathcal{A}} m_{\mathcal{A}^{\otimes Y / \phi}}\left(\mathbf{1}_{\mathcal{A} \otimes Y / \phi}, \operatorname{Sht}_{\mathcal{M}, F_{\mathcal{M}}, \text { univ }}\right) \simeq \operatorname{Sht}_{\mathcal{M}, F_{\mathcal{M}}, Y / \phi, \emptyset}
$$

is given by the excursion

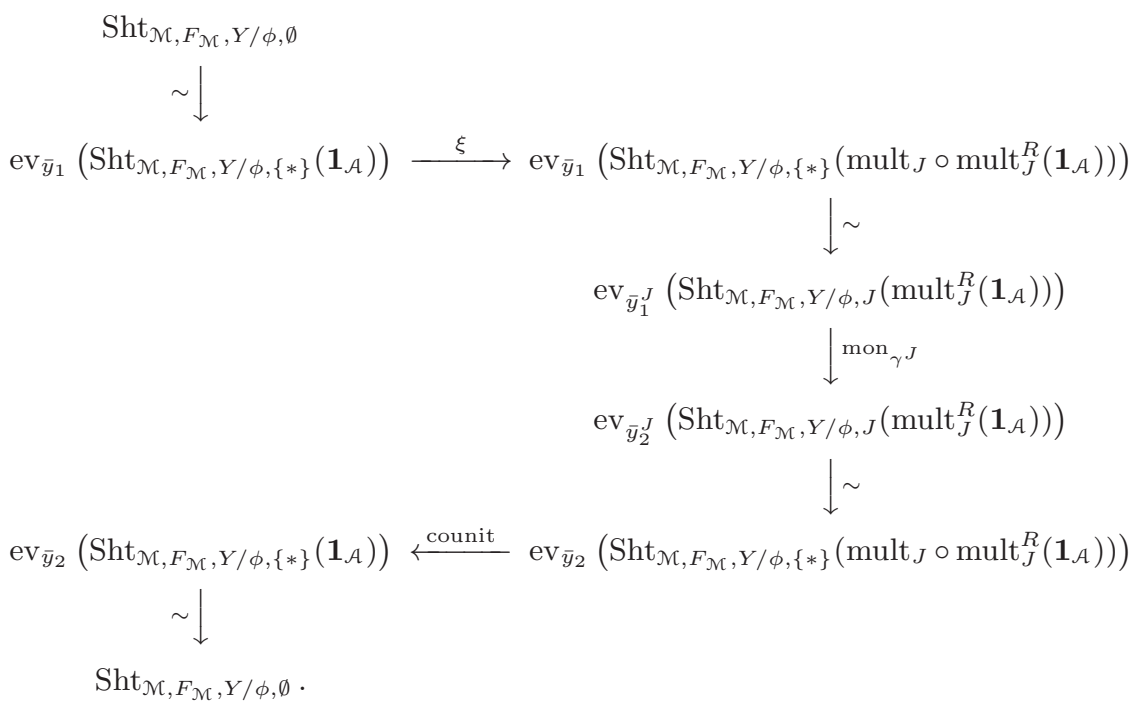


5.5. The "S=T" identity, vacuum case. We are now coming to what is perhaps in the most interesting part in the entire story.

5.5.1. Let $y_{0} \in Y$ be a $\phi$-fixed point. Fix a compact object $a \in \mathcal{A}$. Note that the object

$$
a_{y_{0}} \in \mathcal{A}^{\otimes Y}
$$

is equipped with a natural isomorphism

$$
\alpha_{\text {taut }}: a_{y_{0}} \stackrel{\sim}{\rightarrow} \phi_{*}\left(a_{y_{0}}\right) .
$$

Consider the corresponding natural transformation (in fact, an isomorphism)

$$
\alpha_{a_{y_{0}}, \mathcal{M}, F_{\mathcal{M}}, \text { taut }}: H_{a_{y_{0}}} \circ F_{\mathcal{M}} \stackrel{\sim}{\rightarrow} F_{\mathcal{M}} \circ H_{a_{y_{0}}}
$$

see 3.15).

Hence we can consider the endomorphism

$$
\operatorname{Tr}\left(H_{a_{y_{0}}}, \alpha_{a_{y_{0}}, \mathcal{M}, F_{\mathcal{M}}, \text { taut }}\right): \operatorname{Tr}\left(F_{\mathcal{M}}, \mathcal{M}\right) \rightarrow \operatorname{Tr}\left(F_{\mathcal{M}}, \mathcal{M}\right) .
$$

Remark 5.5.2. We should think of

$$
\operatorname{Tr}\left(H_{a_{y_{0}}}, \alpha_{a_{y_{0}}, \mathcal{M}, F_{\mathcal{M}}, \text { taut }}\right)
$$

as an analogue of the Hecke operator acting on the space of automorphic functions, corresponding to a finite-dimensional representation of $\breve{G}$ (thought of as $a \in \operatorname{Rep}(\breve{G})$ ) applied at a rational point of the curve (thought of as $y_{0}$ ). So, this is V. Lafforgue's "T" operator.

5.5.3. Let $\bar{y}_{0}$ be the projection of $y_{0}$ to $Y / \phi$. The fact that $y_{0}$ was $\phi$-invariant defines a tautological point

Let $\xi_{a}$ be the map

$$
\gamma_{\text {taut }} \in \Omega\left(Y / \phi, \bar{y}_{0}\right)
$$

$$
\mathbf{1}_{\mathcal{A}} \rightarrow \text { mult o mult }{ }^{R}\left(\mathbf{1}_{\mathcal{A}}\right)
$$

defined by $a$, see (4.14).

5.5.4. We claim:

Theorem 5.5.5. The endomorphism $\operatorname{Tr}\left(H_{a_{y_{0}}}, \alpha_{a_{y_{0}}, \mathcal{M}, F_{\mathcal{M}}, \text { taut }}\right)$ of

$$
\operatorname{Tr}\left(F_{\mathcal{M}}, \mathcal{M}\right) \simeq \operatorname{Sht}_{\mathcal{M}, F_{\mathcal{M}}, Y / \phi, \emptyset}
$$

equals the operator (5.8) for $\left(\bar{y}_{0}, J=\{*\} \sqcup\{*\}, \gamma_{\text {taut }}^{+}, \xi_{a}\right)$, where

$$
\gamma_{\text {taut }}^{+}=\left(\gamma_{\text {taut }}, \gamma_{\text {triv }}\right) \text {. }
$$

Remark 5.5.6. We observe that by Proposition 5.3.3. the operator (5.8) for $\left(\bar{y}_{0}, J=\{*\} \sqcup\{*\}, \gamma_{\text {taut }}^{+}, \xi_{a}\right)$, appearing in Theorem 5.5.5 is given explicitly by

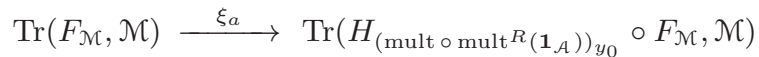

$$
\begin{aligned}
& \downarrow \sim \\
& \operatorname{Tr}\left(H_{\left(\operatorname{mult}^{R}\left(\mathbf{1}_{\mathcal{A}}\right)\right)_{y_{0}, y_{0}}} \circ F_{\mathcal{M}}, \mathcal{M}\right) \\
& \downarrow \text { partial Frobenius, i.e., isomorphism } 5.7
\end{aligned}
$$

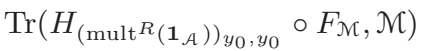

$$
\begin{aligned}
& \downarrow \sim \\
& \operatorname{Tr}\left(F_{\mathcal{M}}, \mathcal{M}\right) \stackrel{\text { counit }}{\longleftarrow} \operatorname{Tr}\left(H_{\left(\text {mult } \circ \operatorname{mult}^{R}\left(\mathbf{1}_{\mathcal{A}}\right)\right)_{y_{0}}} \circ F_{\mathcal{M}}, \mathcal{M}\right) .
\end{aligned}
$$

Remark 5.5.7. Note that the operator (5.10) is an analog of V. Lafforgue's "S" operator. For this reason, we view Theorem 5.5.5 as the toy model for V. Lafforgue's "S=T" statement, i.e., Laf, Proposition $6.2]$. 
Proof of Theorem 5.5.5. First, we note that when considering the endomorphism

$$
\operatorname{Tr}\left(H_{a_{y_{0}}}, \alpha_{a_{y_{0}}, \mathcal{M}, F_{\mathcal{M}}, \text { taut }}\right)
$$

of $\operatorname{Tr}\left(F_{\mathcal{M}}, \mathcal{M}\right)$, we can replace the original $Y$ by $\{*\}$ (with the necessarily trivial endomorphism $\phi$ ) via

$$
\{*\} \stackrel{y_{0}}{\longrightarrow} Y
$$

Next, we claim that when considering the operator (5.8) for

$$
\left(\bar{y}_{0}, J=\{*\} \sqcup\{*\}, \gamma_{\text {taut }}^{+}, \xi_{a}\right),
$$

we can also replace $Y$ by $\{*\}$. Indeed, this immediately follows either from Remark 5.1.9] or from Remark 5.5.6

Hence, in proving the theorem, we can assume that $Y=\{*\}$. Note that

$$
\{*\} / \mathrm{id} \simeq S^{1} .
$$

On the one hand, by Proposition 3.4.6 the endomorphism $\operatorname{Tr}\left(H_{a_{y_{0}}}, \alpha_{a_{y_{0}}, \mathcal{M}, F_{\mathcal{M}}, \text { taut }}\right)$ of $\operatorname{Tr}\left(F_{\mathcal{M}}, \mathcal{M}\right)$ equals the action of the element

$$
\operatorname{cl}\left(a_{y_{0}}, \alpha_{a, \text { taut }}\right) \in \operatorname{Tr}\left(\mathcal{A}^{\otimes \phi}, \mathcal{A}^{\otimes Y}\right),
$$

which in the case $Y=\{*\}$ amounts to the element

$$
\operatorname{cl}(a, \mathrm{id}) \in \operatorname{Tr}(\operatorname{Id}, \mathcal{A})
$$

On the other hand, by Proposition 5.4.3 the operator (5.8) for $\left(\bar{y}_{0}, J=\{*\} \sqcup\{*\}, \gamma_{\text {taut }}^{+}, \xi_{a}\right)$ is given by the action of

$$
\operatorname{Exc}_{\text {univ }}\left(\gamma_{\text {taut }}^{+}, \xi_{a}\right) \in \mathcal{E} n d_{\mathcal{A} \otimes Y / \phi}\left(\mathbf{1}_{\mathcal{A} \otimes Y / \phi}\right),
$$

which in our case is the element

$$
\operatorname{Exc}_{\text {univ }}\left(\gamma_{\text {taut }}^{+}, \xi_{a}\right) \in \mathcal{E} n d_{\mathcal{A} \otimes S^{1}}\left(\mathbf{1}_{\mathcal{A} \otimes S^{1}}\right)
$$

in the notations of Proposition 4.3.5.

Hence, we need to show to show that the above two elements match under the isomorphism

$$
\operatorname{Tr}\left(\mathcal{A}^{\otimes \phi}, \mathcal{A}^{\otimes Y}\right) \simeq \mathcal{E} n d_{\mathcal{A} \otimes Y / \phi}\left(\mathbf{1}_{\mathcal{A} \otimes Y / \phi}\right)
$$

of Theorem 3.8.5 where we identify

$$
\mathcal{A}^{\otimes Y / \phi} \simeq \mathrm{HH}_{\bullet}\left(\mathcal{A}^{\otimes Y}, \mathcal{A}^{\otimes \phi}\right)
$$

Thus, in our case, the identification (5.11) amounts to

$$
\operatorname{Tr}(\operatorname{Id}, \mathcal{A}) \simeq \mathcal{E} n d_{\mathcal{A} \otimes S^{1}}\left(\mathbf{1}_{\mathcal{A} \otimes S^{1}}\right)
$$

Now, the desired identity of elements follows from Corollary 4.3.6.

5.6. The "S=T" identity, general case. As was explained in Remark 5.5.7 the assertion of Theorem 5.5.5 is an analog of V. Lafforgue's $S=T$ identity as operators acting on the space of automorphic functions, i.e., empty-legged shtukas. We will now discuss its generalization, which extends the $S=T$ identity as operators on $I$-legged shtukas. 
5.6.1. Let $y_{0}$ and $a$ be as in Sect. 5.5.1 above. Fix a finite set $J$, a point $\underline{y} \in Y^{J}$ and an object $r \in \mathcal{A}^{\otimes J}$. Denote

$$
J_{+}:=\{*\} \sqcup J, \underline{y}_{+}:=y_{0} \sqcup \underline{y}, J_{++}:=\{*\} \sqcup\{*\} \sqcup J, \underline{y}_{++}:=y_{0} \sqcup y_{0} \sqcup \underline{y} .
$$

The natural transformation (5.9) induces a natural transformation

$$
H_{a_{y_{0}}} \circ H_{r_{\underline{y}}} \circ F_{\mathcal{M}} \simeq H_{r_{\underline{y}}} \circ H_{a_{y_{0}}} \circ F_{\mathcal{M}} \rightarrow H_{r_{\underline{y}}} \circ F_{\mathcal{M}} \circ H_{a_{y_{0}}}
$$

to be denoted $\alpha_{a, \mathcal{M}, F_{\mathcal{M}}, \text { taut }, r_{\underline{y}}}$.

Then we can consider the resulting endomorphism

$$
\operatorname{Tr}\left(H_{a_{y_{0}}}, \alpha_{a, \mathcal{M}, F_{\mathcal{M}}, \text { taut }, r_{\underline{y}}}\right): \operatorname{Tr}\left(H_{r_{\underline{y}}} \circ F_{\mathcal{M}}, \mathcal{M}\right) \rightarrow \operatorname{Tr}\left(H_{r_{\underline{y}}} \circ F_{\mathcal{M}}, \mathcal{M}\right) .
$$

Remark 5.6.2. The map (5.12) is an analog of V. Lafforgue's " $T$ " operator acting on the cohomology of shtukas.

5.6.3. Let $\underline{y}$ be the projection of $\underline{y}$ to $(Y / \phi)^{J}$. By Proposition 5.2.4 we have an identification

$$
\operatorname{Tr}\left(H_{r_{\underline{y}}} \circ F_{\mathcal{M}}, \mathcal{M}\right) \simeq \operatorname{ev}_{\underline{y}}\left(\operatorname{Sht}_{\mathcal{M}, F_{\mathcal{M}}, Y, J}^{\prime}(r)\right) \simeq \operatorname{ev}_{\underline{\underline{y}}}\left(\operatorname{Sht}_{\mathcal{M}, F_{\mathcal{M}}, Y / \phi, J}(r)\right) .
$$

5.6.4. Set $\underline{\bar{y}}_{+}:=\bar{y}_{0} \sqcup \underline{\bar{y}}$ and $\underline{\bar{y}}_{++}:=\bar{y}_{0} \sqcup \bar{y}_{0} \sqcup \underline{\bar{y}}$. Then we can consider the endomorphism of $\mathrm{ev}_{\underline{\underline{y}}}\left(\operatorname{Sht}_{\mathcal{M}, F_{\mathcal{M}}, Y, J}(r)\right)$ equal to the composite

$$
\begin{aligned}
& \operatorname{ev}_{\underline{\bar{y}}}\left(\operatorname{Sht}_{\mathcal{M}, F_{\mathcal{M}}, Y / \phi, J}(r)\right) \longrightarrow \quad \operatorname{ev}_{\underline{\bar{y}}_{+}}\left(\operatorname{Sht}_{\mathcal{M}, F_{\mathcal{M}}, Y / \phi, J_{+}}\left(r \otimes \mathbf{1}_{\mathcal{A}}\right)\right) \\
& \xi_{a} \downarrow \\
& \operatorname{ev}_{\underline{y}_{+}}\left(\operatorname{Sht}_{\mathcal{M}, F_{\mathcal{M}}, Y / \phi, J_{+}}\left(r \otimes \text { mult } \circ \operatorname{mult}^{R}\left(\mathbf{1}_{\mathcal{A}}\right)\right)\right) \\
& \downarrow \sim \\
& \operatorname{ev}_{\underline{\bar{y}}_{++}}\left(\operatorname{Sht}_{\mathcal{M}, F_{\mathcal{X}}, Y / \phi, J_{++}}\left(r \otimes \operatorname{mult}^{R}\left(\mathbf{1}_{\mathcal{A}}\right)\right)\right) \\
& \downarrow \text { mon }_{\gamma_{\text {taut }}, \gamma_{\text {triv }}, \gamma_{\text {triv }}^{J}}^{J} \\
& \operatorname{ev}_{\underline{\bar{y}}_{++}}\left(\operatorname{Sht}_{\mathcal{M}, F_{\mathcal{M}}, Y / \phi J_{++}}\left(r \otimes \operatorname{mult}^{R}\left(\mathbf{1}_{\mathcal{A}}\right)\right)\right) \\
& \downarrow \sim \\
& \operatorname{ev}_{\underline{\bar{y}}_{+}}\left(\operatorname{Sht}_{\mathcal{M}, F_{\mathcal{X}}, Y / \phi, J_{+}}\left(r \otimes \text { mult } \circ \operatorname{mult}^{R}\left(\mathbf{1}_{\mathcal{A}}\right)\right)\right) \\
& \text { counit } \downarrow \\
& \operatorname{ev}_{\underline{\underline{y}}}\left(\operatorname{Sht}_{\mathcal{M}, F_{\mathcal{M}}, Y / \phi, J}(r)\right) \longleftarrow \quad \operatorname{ev}_{\underline{\bar{y}}_{+}}\left(\operatorname{Sht}_{\mathcal{M}, F_{\mathcal{M}}, Y / \phi, J_{+}}\left(r \otimes \mathbf{1}_{\mathcal{A}}\right)\right)
\end{aligned}
$$

Remark 5.6.5. Note that by Propositions 5.2.4 and 5.3.3 the operator (5.13) identifies with

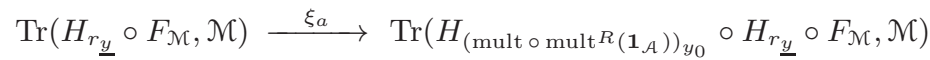

$$
\begin{aligned}
& \downarrow \sim
\end{aligned}
$$

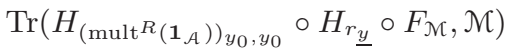

$$
\begin{aligned}
& \downarrow \text { partial Frobenius, i.e., isomorphism } 5.7
\end{aligned}
$$

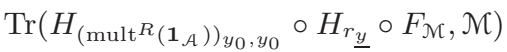

$$
\begin{aligned}
& \downarrow \sim
\end{aligned}
$$

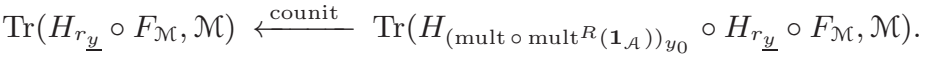

So the operator (5.13) is indeed an analog of V. Lafforgue's "S" operator in the presence of other Hecke functors. 
5.6.6. We claim:

Theorem 5.6.7. The endomorphism $\operatorname{Tr}\left(H_{a_{y_{0}}}, \alpha_{a, \mathcal{M}, F_{\mathcal{M}}, \text { taut }, r_{\underline{y}}}\right)$ of

$$
\operatorname{Tr}\left(H_{r_{\underline{y}}} \circ F_{\mathcal{M}}, \mathcal{M}\right) \simeq \operatorname{ev}_{\underline{y}}\left(\operatorname{Sht}_{\mathcal{M}, F_{\mathcal{M}}, Y / \phi, J}(r)\right)
$$

equals the operator (5.13).

Proof. We claim that Theorem 5.6.7 is in fact a formal corollary of Theorem 5.5.5. Namely, for our given $\mathcal{M}$ set

$$
F_{\mathcal{M}, r_{\underline{y}}}:=H_{r_{\underline{y}}} \circ F_{\mathcal{M}} .
$$

We claim that the identity stated in Theorem 5.6.7 specializes to the identity of Theorem 5.5.5 for the endomorphism $F_{\mathcal{M}, r_{\underline{y}}}$.

Indeed, we have an isomorphism

$$
\operatorname{Tr}\left(H_{r_{\underline{y}}} \circ F_{\mathcal{M}}, \mathcal{M}\right) \simeq \operatorname{Tr}\left(F_{\mathcal{M}, r_{\underline{y}}}, \mathcal{M}\right),
$$

and under this identification, the endomorphism $\operatorname{Tr}\left(H_{a_{y_{0}}}, \alpha_{a, \mathcal{M}}, F_{\mathcal{M}}\right.$, taut, $\left.r_{\underline{y}}\right)$ of the LHS corresponds to

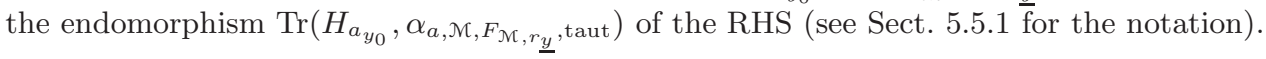

It therefore suffices to show that there exists an isomorphism

$$
\operatorname{ev}_{\underline{\bar{y}}}\left(\operatorname{Sht}_{\mathcal{M}, F_{\mathcal{M}}, Y / \phi, J}(r)\right) \simeq \operatorname{Sht}_{\mathcal{M}, F_{\mathcal{M}, r_{\underline{y}}}, Y / \phi, \emptyset}
$$

such that the endomorphism (5.13) of the LHS corresponds to the endomorphism (5.8) with

$$
\left(\bar{y}_{0}, J=\{*\} \sqcup\{*\}, \gamma_{\text {taut }}^{+}, \xi_{a}\right)
$$

of the RHS.

It follows from Theorem 4.4.6 that there exists a canonical isomorphism

$$
\iota\left(r_{\underline{y}}\right) \otimes \operatorname{Sht}_{\mathcal{M}, F_{\mathcal{M}}, \text { univ }} \simeq \operatorname{Sht}_{\mathcal{M}, F_{\mathcal{M}, r_{\underline{y}}}, \text { univ }}
$$

of objects of $\mathcal{A}^{\otimes Y / \phi}$. Therefore using explicit formulas of Sect. 1.8.5 for every finite set $I$, a point $\underline{\bar{y}}^{\prime} \in Y^{I}$ and an object $r^{\prime} \in \mathcal{A}^{\otimes I}$, we have an isomorphism

$$
\operatorname{ev}_{\underline{\bar{y}} \sqcup \underline{\bar{y}}^{\prime}}\left(\operatorname{Sht}_{\mathcal{M}, F_{\mathcal{M}}, Y / \phi, J \sqcup I}\left(r \otimes r^{\prime}\right)\right) \simeq \operatorname{ev}_{\underline{\bar{y}}^{\prime}}\left(\operatorname{Sht}_{\mathcal{M}, F_{\mathcal{M}, r_{\underline{y}}}, Y / \phi, I}\left(r^{\prime}\right)\right),
$$

functorial in $\underline{y}^{\prime}$ and $r^{\prime}$.

Applying this isomorphism in the particular case $I=\emptyset$ and $r^{\prime}=\mathbf{1}_{\text {Vect }}$, we get the isomorphism (5.15) we need.

\section{Appendix A. Sheaves And singular support}

This appendix is included for the sake of completeness. We will review the notion of singular support in different sheaf-theoretic contexts.

The main result of this section is Theorem A.3.9 which says the following: In the product situation $y \times X$, where $y$ is an algebraic stack and $X$ a proper scheme, the category of sheaves on $y \times X$ whose singular support is of the form

$$
\mathcal{N}^{\prime}:=\mathcal{N} \times\{\text { zero section }\} \subset T^{*}(y) \times T^{*}(X)=T^{*}(y \times X),
$$

is equivalent to the tensor product category

$$
\operatorname{Shv\mathcal {N}}_{(y)}\left(\operatorname{Shv}_{\text {lisse }}(X) .\right.
$$

\section{A.1. Sheaf-theoretic contexts.}


A.1.1. In this section and the next, we will take $\operatorname{Shv}(-)$ to be any of the following sheaf-theoretic contexts:

(a) $\operatorname{Shv}_{\mathrm{cl}}(S)$, the category of all sheaves in the classical topology with coefficients in e, for $S$ an affine scheme over $\mathbb{C}$;

(a') $\operatorname{Shv}(S)=\operatorname{Ind}\left(\operatorname{Shv}_{\mathrm{cl} \text {, constr }}(S)\right)$, where $\operatorname{Shv}_{\mathrm{cl} \text {,constr }}(S) \subset \operatorname{Shv}_{\mathrm{cl}}(S)$ is the (small) subcategory consisting of constructible sheaves;

(b) $\operatorname{Shv}(S)=\mathrm{D}-\bmod (S)$, for $S$ an affine scheme over a ground field $k$ of characteristic 0 ;

(b') $\operatorname{Shv}(S)=\operatorname{Ind}\left(\mathrm{D}-\bmod _{\text {hol }}(S)\right)$, where D- $\bmod _{\text {hol }}(S) \subset \mathrm{D}$-mod$(S)$ is the (small) subcategory consisting of holonomic D-modules;

(b") $\operatorname{Shv}(S)=\operatorname{Ind}\left(\mathrm{D}-\bmod _{\text {hol, RS }}(S)\right.$ ), where $\mathrm{D}-\bmod _{\text {hol, RS }}(S) \subset \mathrm{D}-\bmod (S)$ is the (small) subcategory consisting of holonomic D-modules with regular singularities;

(c) $\operatorname{Shv}(S):=\operatorname{Shv}_{\mathbb{Z} / \ell^{n} \text {,et }}(S)$, the category of all étale sheaves on $S$ with coefficients in $\mathbb{Z} / \ell^{n} \mathbb{Z}$, for $S$ an affine scheme over any ground field of characteristic prime to $\ell$. Note that we have

$$
\operatorname{Shv}_{\mathbb{Z} / \ell^{n}, \text { et }}(S) \simeq \operatorname{Ind}\left(\operatorname{Shv}_{\mathbb{Z} / \ell^{n}, \text { et,constr }}(S)\right),
$$

where $\operatorname{Shv}_{\mathbb{Z} / \ell^{n}, \text { et,constr }}(S) \subset \operatorname{Shv}_{\mathbb{Z} / \ell^{n}, \text { et }}(S)$ is the full subcategory of constructible sheaves, see GaLu Proposition 2.2.6.2];

(d) $\operatorname{Shv}(S):=\operatorname{Ind}\left(\operatorname{Shv}_{\mathbb{Z}_{\ell}, \text { et,constr }}(S)\right)$, where $\operatorname{Shv}_{\mathbb{Z}_{\ell}, \text { et,constr }}(S)$ is the category of constructible $\ell$-adic sheaves on $S$ (see GaLu, Defn. 2.3.2.1]), which is equivalent to

$$
\lim _{n} \operatorname{Shv}_{\mathbb{Z} / \ell^{n}, \text { et,constr }}(S)
$$

(d') $\operatorname{Shv}(S):=\operatorname{Ind}\left(\operatorname{Shv}_{\mathbb{Q}_{\ell}, \text { et,constr }}(S)\right)$, where $\operatorname{Shv}_{\mathbb{Q}_{\ell}, \text { et,constr }}(S)$ is obtained from $\operatorname{Shv}_{\mathbb{Z}_{\ell}, \text { et,constr }}(S)$ by inverting $\ell$. Note that

$$
\operatorname{Ind}\left(\operatorname{Shv}_{\mathbb{Q}_{\ell}, \text { et }, \text { constr }}(S)\right) \simeq \operatorname{Ind}\left(\operatorname{Shv}_{\mathbb{Z}_{\ell}, \text { et }, \text { constr }}(S)\right) \underset{\mathbb{Z}_{\ell}}{\otimes} \mathbb{Q}_{\ell}
$$

A.1.2. In what follows, we will refer to the cases (a') and (b')-(d') as "ind-constructible".

Note that, by definition, in these cases, the category $\operatorname{Shv}(S)$ is compactly generated. In particular, it is dualizable.

A.1.3. Let $y$ be a prestack. In all cases apart from (a), we define $\operatorname{Shv}(y)$ as

$$
\lim _{S \in\left(\operatorname{Sch}_{/ y}^{\text {aff }}\right) \text { op }} \operatorname{Shv}(S)
$$

where for $S_{1} \stackrel{f}{\rightarrow} S_{2}$, the corresponding functor $\operatorname{Shv}\left(S_{2}\right) \rightarrow \operatorname{Shv}\left(S_{1}\right)$ is $f^{!}$. In the above formula, $\operatorname{Sch}_{/ y}^{\text {aff }}$ is the category of affine schemes over $y$.

Note that in all cases apart from (b), the functor $f^{!}$admits a left adjoint, namely $f_{!}$. Hence, from Lemma 1.4.8 (b), we obtain that in all of these cases, we can write $\operatorname{Shv}(y)$ also as

$$
\underset{S \in \operatorname{Sch}_{/ y}^{\text {aff }}}{\operatorname{colim}} \operatorname{Shv}(S)
$$

where for $S_{1} \stackrel{f}{\rightarrow} S_{2}$, the corresponding functor $\operatorname{Shv}\left(S_{1}\right) \rightarrow \operatorname{Shv}\left(S_{2}\right)$ is $f_{!}$.

Note also that in the ind-constructible contexts (i.e., cases (a') and (b')-(d')), we obtain from (A.2) that the category $\operatorname{Shv}(y)$ is compactly generated (since each $\operatorname{Shv}(S)$ is). In particular, $\operatorname{Shv}(y)$ is dualizable. 
A.1.4. Let us now consider case (a). (The slight glitch here is that in this case the functors $f^{!}$are no longer continuous.) We define $\operatorname{Shv}(y)$ by formula (A.2), where the colimit is taken DGCat.

Let DGCat ${ }^{\text {discont }}$ denote the category whose objects are not necessarily cocomplete DG categories, and we allow all (exact) functors between such categories. Specifically, DGCat ${ }^{\text {discont }}$ is the category of

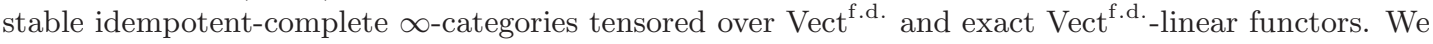
have a tautological functor

$$
\text { DGCat } \rightarrow \text { DGCat }{ }^{\text {discont }}
$$

We can also form the limit A.1, taking place in DGCat ${ }^{\text {discont }}$. Now, according to GR1 Corollary 5.3.4(b)] (which is a generalization of Lemma 1.4.8 to the case when the right adjoints are not necessarily continuous), the image of $\operatorname{Shv}(y)$, which is defined by formula (A.2), under the forgetful functor (A.3) identifies with the limit A.10.

In other words, we have a canonical isomorphism

$$
\operatorname{Shv}_{\mathrm{cl}}(y) \simeq \lim _{S \in \operatorname{Sch}_{/ y}^{\mathrm{aff}}} \operatorname{Shv}_{\mathrm{cl}}(S)
$$

as objects of DGCat ${ }^{\text {discont }}$.

A.1.5. Let now $y$ be an algebraic stack. In this case, we can consider the category $\operatorname{Sch}_{/ y, s m}^{\text {aff }}$, consisting of affine schemes equipped with a smooth map to $y$, and whose morphisms are smooth maps between affine schemes over $y$.

A smooth descent argument shows that the restriction functor

$$
\lim _{S \in \operatorname{Sch}_{/ y}^{\text {aff }}} \operatorname{Shv}(S) \rightarrow \lim _{S \in \operatorname{Sch}_{/ y, s m}^{\text {aff }}} \operatorname{Shv}(S)
$$

is an equivalence.

Hence, for an algebraic stack $y$, we have

$$
\operatorname{Shv}(y) \simeq \lim _{S \in \operatorname{Sch}_{/ y}^{\text {aff }}, \mathrm{sm}} \operatorname{Shv}(S)
$$

where this isomorphism takes place in DGCat ${ }^{\text {discont }}$ in context (a) and in DGCat in other contexts.

Remark A.1.6. The presentation of $\operatorname{Shv}(y)$ given by (A.4) as an object of DGCat is valid also in case (a): indeed, since smooth pullbacks $f^{!}$are continuous also in case (a), the assertion follows from (A.4) and the fact that the tautological functor DGCat $\rightarrow$ DGCat ${ }^{\text {discont }}$ preserves limits, and for $\mathcal{C}_{1}, \mathcal{C}_{2} \in$ DGCat, the map induced by forgetful functor

$$
\operatorname{Maps}_{\text {DGCat }}\left(\mathcal{C}_{1}, \mathcal{C}_{2}\right) \rightarrow \operatorname{Maps}_{\text {DGCat }}{ }^{\text {discont }}\left(\mathfrak{C}_{1}, \varrho_{2}\right)
$$

induces an isomorphism on the connected components corresponding to equivalences.

A.1.7. Let Corr(PreStk)ind-sch,all be the category of correspondences as in GR2, Chapter 3, Sect. 5.4], whose objects are prestacks $y$, and where morphisms from $y_{1}$ to $y_{2}$ are diagrams

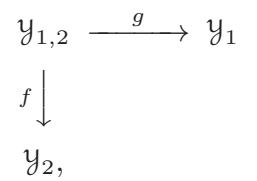

where $g$ any map, and $f$ is required to be ind-schematic. The composition of A.5 and

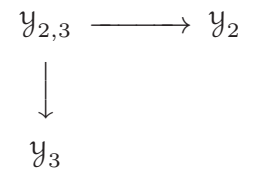


is given by

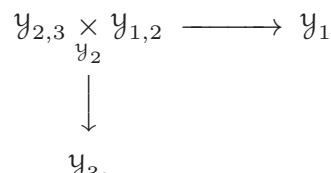

Let us first exclude the context (a). Then the construction of loc.cit. applies, and we can extend $\operatorname{Shv}(-)$ to a functor

$$
\text { Shveorr : Corr(PreStk) })_{\text {ind-sch,all }} \rightarrow \text { DGCat . }
$$

At the level of objects this functor sends $y \mapsto \operatorname{Shv}(y)$. At the level of morphisms, this functor sends a morphism (A.5) to the functor

$$
f_{*} \circ g^{!}: \operatorname{Shv}\left(y_{1}\right) \rightarrow \operatorname{Shv}\left(y_{2}\right)
$$

Compatibility with compositions is insured by base change.

Furthermore, the functor $\mathrm{Sh}_{\text {Corr }}$ possesses a natural right-lax symmetric monoidal structure, see GR2, Chapter 3, Sect. 6.1], where Corr(PreStk)ind-sch,all is a symmetric monoidal category with respect to the level-wise product.

A.1.8. Let us now consider the context (a). In this case we will consider Shvcorr as taking values in DGCat ${ }^{\text {discont. }}$. We will regard Shv ${ }_{\text {Corr }}$ as equipped with the right-lax symmetric monoidal structure, with respect to the following symmetric monoidal structure on DGCat ${ }^{\text {discont}}$ :

For $\mathcal{C} \in$ DGCat $^{\text {discont }}$, we let

$$
\text { Funct }_{\text {DGCatdiscont }}\left(\mathcal{C}_{1} \stackrel{\text { discont }}{\otimes} \mathcal{C}_{2}, \mathcal{C}\right)
$$

consist of all bi-exact bi-e-linear (but not necessarily bi-continuous) functors

$$
\mathcal{C}_{1} \times \mathcal{C}_{2} \rightarrow \mathcal{C}
$$

More formally, we apply the construction of [GR1, Chapter 1, Sect. 6.1.1], but dropping the continuity condition.

Remark A.1.9. Note that given an object $\mathcal{C} \in$ DGCat, the space of structures of associative (resp., commutative) algebras on it within DGCat embeds fully faithfully into the space of such structures within DGCat ${ }^{\text {discont }}$.

The same applies to actions of a given monoidal DG category on another DG category.

\section{A.2. Sheaves on a product.}

A.2.1. Note that for a pair of affine schemes, we have a naturally defined functor, given by external tensor product

$$
\operatorname{Shv}\left(S_{1}\right) \otimes \operatorname{Shv}\left(S_{2}\right) \rightarrow \operatorname{Shv}\left(S_{1} \times S_{2}\right), \quad \mathcal{F}_{1}, \mathcal{F}_{2} \mapsto \mathcal{F}_{1} \otimes \mathcal{F}_{2}
$$

This functor is an equivalence in case (b): for a pair of associative algebras $A_{1}$ and $A_{2}$, the functor

$$
A_{1}-\bmod \otimes A_{2}-\bmod \rightarrow\left(A_{1} \otimes A_{2}\right)-\bmod , \quad M_{1}, M_{2} \mapsto M_{1} \otimes M_{2}
$$

is an equivalence (see Lu2, Theorem 4.8.5.16]). 
A.2.2. The following is known (the assertion is valid for any pair of locally compact Hausdorff topological spaces, see [Lu1, Theorem 7.3.3.9, Prop. 7.3.1.11] and [Lu2, Prop. 4.8.1.17]):

Theorem A.2.3. The functor (A.6) is an equivalence in case (a).

Remark A.2.4. It follows from Theorem A.2.3 that for a locally compact topological space $M$, the functors

$$
\operatorname{Vect} \stackrel{\mathrm{e}_{M}}{\rightarrow} \operatorname{Shv}(M) \stackrel{\Delta_{1}}{\rightarrow} \operatorname{Shv}(M \times M) \simeq \operatorname{Shv}(M) \otimes \operatorname{Shv}(M)
$$

and

$$
\operatorname{Shv}(M) \otimes \operatorname{Shv}(M) \rightarrow \operatorname{Shv}(M \times M) \stackrel{\Delta^{*}}{\rightarrow} \operatorname{Shv}(M) \stackrel{\mathrm{C}_{c}^{\bullet}(M,-)}{\longrightarrow} \text { Vect }
$$

define an identification

$$
\operatorname{Shv}(M)^{\vee} \simeq \operatorname{Shv}(M)
$$

Note, however, that as was shown by A. Neeman (see [Ne1]), for a topological manifold $M$, the category $\operatorname{Shv}(M)$ is not compactly generated, unless $M$ is discrete. $\operatorname{So} \operatorname{Shv}(M)$ is an example of a dualizable but not compactly generated category.

A.2.5. In the ind-constructible contexts, the functor A.6 fails to be an equivalence. However, we have:

Lemma A.2.6. In the constructible contexts the functor A.6 is fully faithful.

Proof. For a pair of DG categories $\mathcal{C}_{1}, \mathcal{C}_{2}$ and

$$
c_{i}^{\prime} \in \mathcal{C}_{i}^{c}, c_{i}^{\prime \prime} \in \mathcal{C}_{i}, i=1,2,
$$

the map

$$
\mathcal{H}_{o m} \mathrm{e}_{1}\left(c_{1}^{\prime}, c_{1}^{\prime \prime}\right) \otimes \mathcal{H}_{o m} \mathrm{e}_{2}\left(c_{2}^{\prime}, c_{2}^{\prime \prime}\right) \rightarrow \mathcal{H}_{o m} \mathrm{e}_{1} \otimes \mathfrak{e}_{2}\left(c_{1}^{\prime} \otimes c_{2}^{\prime}, c_{1}^{\prime \prime} \otimes c_{2}^{\prime \prime}\right)
$$

is an isomorphism (see GR1, Chapter 1, Proposition 7.4.2]).

Since the tensor product of compactly generated categories is compactly generated by tensor products of compact objects (see GR1, Chapter 1, Proposition 7.4.2]), in order to prove the lemma, it suffices to show that for

$$
\mathcal{F}_{i}^{\prime}, \mathcal{F}_{i}^{\prime \prime} \in \operatorname{Shv}\left(S_{i}\right)^{c}, \quad i=1,2
$$

the map

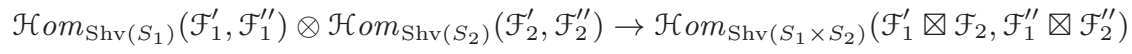

is an isomorphism. However, this follows from Kunneth's formula.

A.2.7. We now consider the case of prestacks. Again, external tensor product gives rise to a functor

$$
\operatorname{Shv}\left(y_{1}\right) \otimes \operatorname{Shv}\left(y_{2}\right) \rightarrow \operatorname{Shv}\left(y_{1} \times y_{2}\right), \quad \mathcal{F}_{1}, \mathcal{F}_{2} \mapsto \mathcal{F}_{1} \otimes \mathcal{F}_{2}
$$

The argument in GR1, Chapter 3, Theorem 3.1.7] shows:

Lemma A.2.8. In case (b), if one of the categories $\operatorname{Shv}\left(y_{i}\right)$ is dualizable, then the functor (A.7) is an equivalence.

In addition, from Theorem A.2.3 and GR1, Chapter 3, Equation (3.4)], we obtain:

Corollary A.2.9. The functor (A.7) is an equivalence in case (a).

Finally, we claim:

Proposition A.2.10. The functor A.7 is fully faithful in the ind-constructible contexts. 
Proof. Since Shv $\left(y_{i}\right)$ are dualizable (see Sect. A.1.3), the argument in GR1, Chapter 3, Theorem 3.1.7] shows that we can write

$$
\operatorname{Shv}\left(y_{1}\right) \otimes \operatorname{Shv}\left(y_{2}\right)
$$

as

$$
\lim _{S_{1} \rightarrow y_{1}, S_{2} \rightarrow y_{2}} \operatorname{Shv}\left(S_{1}\right) \otimes \operatorname{Shv}\left(S_{2}\right)
$$

and by GR1, Chapter 3, Equation (3.4)], we have

$$
\operatorname{Shv}\left(y_{1} \times y_{2}\right) \simeq \lim _{S_{1} \rightarrow y_{1}, S_{2} \rightarrow y_{2}} \operatorname{Shv}\left(S_{1} \times S_{2}\right) .
$$

Hence, the assertion follows from Lemma A.2.6

\section{A.3. Singular support.}

A.3.1. Let $S$ be an affine scheme. First, we assume that $S$ is smooth. Let $\mathcal{N} \subset T^{*}(S)$ be a conical Zariski-closed subset. In each of our sheaf-theoretic contexts we can single out a full subcategory

$$
\operatorname{Shv\mathcal {N}}_{\mathcal{N}}(S) \subset \operatorname{Shv}(S)
$$

consisting of objects with singular support contained in $\mathcal{N}$ :

-In case (a), we require that each cohomology sheaf belongs to $\operatorname{Shv}_{\mathrm{cl}, \mathfrak{N}}(S)$, where the latter is defined in [KS, Sect. 8];

-In case (a'), we inherit the definition from case (a) for $\operatorname{Shv}_{\mathrm{cl} \text {, constr }}(S)$, and then ind-extend (alternatively, transfer the definition via the Riemann-Hilbert from case (b")).

-In case (b), we take the ind-completion of the category D- $\bmod _{\mathcal{N}}(S)^{\mathrm{f} \cdot \mathrm{g}} \subset \mathrm{D}-\bmod (S)^{\mathrm{f} \cdot \mathrm{g}}$, obtained by requiring that each cohomology belong to $\mathrm{D}-\bmod _{\mathcal{N}}(S)^{\text {f.g., } \mathcal{O}}$, where the latter is the standard D-module notion;

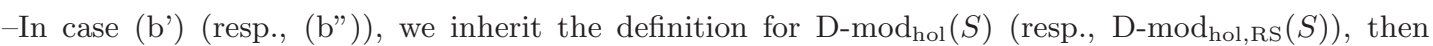
ind-extend.

-In cases (c), we give the definition for $\operatorname{Shv}_{\mathbb{Z} / \ell^{n} \text {,et,constr }}(S)$ following $[\mathrm{Be}$, then ind-extend.

-In case (d) we will say that an object of $\operatorname{Shv}_{\mathbb{Z}_{\ell}, \text { et,constr }}(S)$ has singular support in $\mathcal{N}$ if its projection $\bmod \ell$ does (alternatively, we apply the definition of $[\mathrm{Be}]$ directly to $\operatorname{Shv}_{\mathbb{Z}_{\ell} \text {, et,constr }}(S)$ ), then ind-extend;

-In case (d') we apply the definition of $[\mathrm{Be}]$ to $\left.\operatorname{Shv}_{\mathbb{Q}_{\ell}, \text { et,constr }}(S)\right)$, then ind-extend.

Remark A.3.2. Note that in all cases apart from (a), there exists another (a priori, larger) full subcategory of $\operatorname{Shv}(S)$, to be denoted $\operatorname{Shv}_{\mathcal{N}}(S)$, that one could call "sheaves with singular support in $\mathcal{N}$ ". Namely, an object belongs to $\operatorname{Shv}_{\hat{\mathcal{N}}}(S)$ if its cohomology sheaves (with respect to the perverse t-structure) belong to $\operatorname{Shv}_{\mathcal{N}}(S)$.

One can show that $\operatorname{Shv}_{\mathcal{N}}(S)$ identifies with the left completion of $\operatorname{Shv\mathcal {N}}(S)$ with respect to its tstructure. What is not clear, however, is whether the category $\operatorname{Shv}_{\mathcal{N}}(S)$ is compactly generated.

Note that the embedding $\operatorname{Shv\mathcal {N}}(S) \hookrightarrow \operatorname{Shv}_{\mathcal{N}}(S)$ is not always an equivalence (equivalently, $\operatorname{Shv} \hat{\mathcal{N}}(S)$ is not always generated by objects that are compact in $\operatorname{Shv}(S)$ ). For example, this occurs in the example of $S=\mathbb{P}^{1}$ and $\mathcal{N}$ being the zero-section. 
A.3.3. We will now show how to extend the above definition to the case when $S$ is not necessarily smooth. Let $\mathcal{F}$ be a coherent sheaf on $S$ (in cohomological degree 0 ). Represent $\mathcal{F}$ as

$$
\operatorname{coker}\left(\mathcal{E}_{1} \rightarrow \mathcal{E}_{0}\right),
$$

where $\mathcal{E}_{1}$ and $\mathcal{E}_{0}$ are locally free. Consider the total spaces of $\mathcal{E}_{i}$ as group-schemes over $S$,

$$
\operatorname{Tot}\left(\varepsilon_{i}\right):=\operatorname{Spec}_{S}\left(\operatorname{Sym}_{\mathcal{O}_{S}}\left(\varepsilon_{i}^{\vee}\right)\right) \text {. }
$$

Consider the algebraic stack

$$
\operatorname{Tot}(\mathcal{F}):=\operatorname{Tot}\left(\mathcal{E}_{0}\right) / \operatorname{Tot}\left(\mathcal{E}_{1}\right) \text {. }
$$

The object $\operatorname{Tot}(\mathcal{F})$, viewed as an algebraic stack, depends on the presentation (A.8). But it is well-defined in the localization of the category of algebraic stacks, where we invert morphisms that are smooth, surjective and whose fibers are of the form pt $H$ where $H$ is a vector group.

We have a well-defined notion of a Zariski-closed subset of $\operatorname{Tot}(\mathcal{F})$. For a choice of a presentation (A.8), they bijectively correspond to Zariski-closed subsets of $\operatorname{Tot}\left(\mathcal{E}_{0}\right)$ that are invariant under the action of $\operatorname{Tot}\left(\varepsilon_{1}\right)$. This notion does not depend on the presentation (A.8).

A.3.4. Taking $\mathcal{F}:=\Omega^{1}(S)$, we thus obtain an object $T^{*}(S):=\operatorname{Tot}\left(\Omega^{1}(S)\right)$ in the above localization of the category of algebraic stacks. For a closed embedding

$$
f: S \hookrightarrow S^{\prime}
$$

with $S^{\prime}$ smooth, the codifferential map

$$
S \underset{S^{\prime}}{\times} T^{*}\left(S^{\prime}\right) \rightarrow T^{*}(S)
$$

realizes $T^{*}(S)$ as a quotient of $S \underset{S^{\prime}}{\times} T^{*}\left(S^{\prime}\right)$ by a vector group.

A.3.5. Thus, we have a well-defined notion of (conical) Zariski-closed subset of $T^{*}(S)$. We emphasize that although $T^{*}(S)$ as an algebraic stack depends on some choices, the set of its Zariski-closed subsets does not.

Note also that the cotangent fibers, i.e., the sets $T_{s}^{*}(S)$ for $s \in S(k)$, underlying the fibers of $T^{*}(S)$, are the classical cotangent spaces, and as such do not depend on any choices.

For $\mathcal{N} \subset T^{*}(S)$ let

$$
\mathcal{N}^{\prime} \subset S \underset{S^{\prime}}{\times} T^{*}\left(S^{\prime}\right) \subset T^{*}\left(S^{\prime}\right)
$$

be the preimage of $\mathcal{N}$ under the map A.10. A subset $\mathcal{N}$ as above is completely determined by its fibers

$$
\mathcal{N}_{s}:=\mathcal{N} \cap T_{s}^{*}(S) \subset T_{s}^{*}(S), \quad s \in S(k) .
$$

We say that an object $\mathcal{F} \in \operatorname{Shv}(S)$ has singular support in $\mathcal{N}$ if $f_{*}(\mathcal{F})$ has singular support in $\mathcal{N}^{\prime}$. It is not difficult to verify that this definition does not depend in the choice of the embedding $f: S \rightarrow S^{\prime}$.

We shall say that $\mathcal{N} \subset T^{*}(S)$ is half-dimensional if $\mathcal{N}^{\prime} \subset T^{*}\left(S^{\prime}\right)$ is such for some/any smooth $S^{\prime}$.

A.3.6. Let $y$ be an algebraic stack. For a coherent sheaf $\mathcal{F}$ on $y$ we can talk about Zariski-closed subsets of $\operatorname{Tot}(\mathcal{F})$. Namely, they correspond bijectively to compatible families of Zariski-closed subsets of $\operatorname{Tot}\left(\left.\mathcal{F}\right|_{S}\right)$ for $S \in \operatorname{Sch}_{/ y}^{\text {aff }}$ (or, equivalently, $S \in \operatorname{Sch}_{/ y, s m}^{\text {aff }}$ ).

Taking $\mathcal{F}:=\Omega^{1}(y)$, we thus obtain a well-defined notion of (conical) Zariski-closed subset of $T^{*}(y)$ (note that we are not even trying to define $T^{*}(y)$ itself; that said, as in the case of schemes, the cotangent fibers $T_{y}^{*}(y), y \in y(k)$, are the classical cotangent spaces, and thus are well-defined).

Thus, we can talk about Zariski-closed subsets $\mathcal{N} \subset T^{*}(y)$. As in the case of schemes, such $\mathcal{N}$ is completely determined by the subsets

$$
\mathcal{N}_{y}:=\mathcal{N} \cap T_{y}^{*}(y) \subset T_{y}^{*}(y), \quad y \in y(k) .
$$

To a conical Zariski-closed subset $\mathcal{N} \subset T^{*}(y)$ we associate a full subcategory

$$
\operatorname{Shv\mathcal {N}}(y) \subset \operatorname{Shv}(y) \text {. }
$$


Namely, an object belongs to $\operatorname{Shv}_{\mathcal{N}}(y)$ if for any smooth map $S \rightarrow y$ (for $S$ an affine scheme), its pullback to $S$ belongs to

$$
\operatorname{Shv}_{\mathcal{N}_{S}}(S) \subset \operatorname{Shv}(S)
$$

where $\mathcal{N}_{S}$ is the image of $\underset{y}{\mathcal{N}} \underset{y}{ } S$ under the co-differential

$$
T^{*}(y) \underset{y}{x} S \rightarrow T^{*}(S)
$$

Here we are using the presentation of $\operatorname{Shv}(y)$ as in Sect. A.1.5 (see also Remark A.1.6 in case (a)).

In what follows we shall say that $\mathcal{N} \subset T^{*}(y)$ is half-dimensional if its image is such under (A.11) for some/any smooth cover $S \rightarrow y$.

A.3.7. Let $y$ be a smooth. In this case we define a full subcategory

$$
\operatorname{Shv}_{\text {lisse }}(y) \subset \operatorname{Shv}(y) \text {. }
$$

We first give the definition for affine schemes; for stacks, lisse would mean that the pullback to affine schemes under smooth maps is lisse.

For an affine scheme, we set:

-In case (a), lisse means that each cohomology sheaf is locally constant;

-In case (a'), lisse means a colimit of constructible locally constant objects;

-In cases (b), (b') and (b"), lisse means a colimit of $\mathcal{O}$-coherent objects;

-In case (c), lisse means a colimit of constructible locally constant objects;

-In case (d), lisse means a colimit of objects that are constructible and locally constant (i.e., ones whose reduction $\bmod \ell$ is constructible and locally constant);

-In case (d'), lisse means a colimit of objects that are constructible and locally constant.

We note that in all of the above case, being lisse is equivalent to belonging to $\operatorname{Shv\mathcal {N}}(y)$, where $\mathcal{N}$ is the zero-section.

A.3.8. We are going to prove:

Theorem A.3.9. Let $\mathcal{N} \subset T^{*}(y)$ be half-dimensional. Let $X$ be a smooth scheme, assumed proper in all cases apart from (a), ( $a$ ') and (b"). Set

$$
\mathcal{N}^{\prime}:=\mathcal{N} \times\{\text { zero-section }\} \subset T^{*}(y \times X) .
$$

Then the functor

$$
\operatorname{Shv}_{\mathcal{N}}(y) \otimes \operatorname{Shv}_{\text {lisse }}(X) \rightarrow \operatorname{Shv}_{\mathcal{N}^{\prime}}(y \times X)
$$

is an equivalence.

Remark A.3.10. Note that if our sheaf-theoretic context is $(\mathrm{b})$, the assumption that $\mathcal{N} \subset T^{*}(y)$ is half-dimensional implies that objects from $\mathrm{D}-\bmod _{\mathcal{N}}(y)$ are automatically holonomic, i.e., this puts us into context (b').

\section{A.4. Proof of Theorem A.3.9 in case (a).}


A.4.1. The initial observation is the following:

Let

$$
Z \mapsto Z^{\text {top }}, \quad \mathrm{Sch} \rightarrow \mathrm{Top}
$$

denote the functor that associates to a scheme over $\mathbb{C}$ the topological space underlying the corresponding analytic space.

Let

$$
\mathrm{Y} \rightarrow \mathrm{Y}^{\text {sing }}, \quad \mathrm{Top} \rightarrow \mathrm{Spc}
$$

denote the functor of singular chains.

We will denote the composite functor Sch $\rightarrow$ Spc by

$$
Z \mapsto Z^{\text {top }, \text { sing }}
$$

Taking fibers at the points of $Z^{\text {top }}$ defines a functor

$$
Z^{\text {top,sing }} \times \operatorname{Shv}_{\text {lisse }}(Z) \rightarrow \text { Vect },
$$

i.e., a functor

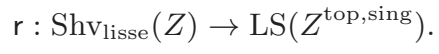

We have:

Lemma A.4.2. The functor A.12 is an equivalence.

Proof. Both categories are equipped with t-structures, in which they are left and right complete, and the functor $r$ is t-exact. Hence, it is enough to show that the functor A.12 is fully faithful on the bounded subcategories and essentially surjective on the hearts.

The former is the expression of the fact that sheaf cohomology of a complex with locally constant cohomology sheaves can be computed via singular cochains.

The latter follows from the fact that both abelian categories in question identify with modules over the fundamental groupoid of $Z^{\text {top }}$.

A.4.3. We proceed with the proof of Theorem A.3.9

Since $\operatorname{Shv}_{\text {lisse }}(X)$ is dualizable (e.g., by Lemma A.4.2 and Proposition 1.4.5(b)), by the argument of GR1, Chapter 3, Theorem 3.1.7], we have:

$$
\operatorname{Shv\mathcal {N}}_{\mathcal{N}}(y) \otimes \operatorname{Shv}_{\text {lisse }}(X) \simeq \lim _{S \in\left(\operatorname{Sch}_{/ y, \mathrm{am}}^{\mathrm{aff}}\right)^{\mathrm{op}}} \operatorname{Shv}_{\mathcal{N}_{S}}(S) \otimes \operatorname{Shv}_{\text {lisse }}(X)
$$

Similarly, a smooth descent argument shows that the functor

$$
\operatorname{Shv}_{\mathcal{N}^{\prime}}(y \times X) \rightarrow \lim _{S \in\left(\operatorname{Sch}_{/ y, s m}^{\text {aff }},{ }^{\text {op }}\right.} \operatorname{Shv}_{\mathcal{N}_{S}^{\prime}}(S \times X)
$$

is an equivalence.

Hence, the assertion of the theorem reduces to the case when $y$ is an (affine) scheme. 
A.4.4. Note that if $\mathcal{C}_{1}$ is dualizable, and $\mathcal{C}_{2}^{\prime} \hookrightarrow \mathcal{C}_{2}$ is a fully faithful embedding, then

$$
\mathrm{C}_{1} \otimes \mathrm{C}_{2}^{\prime} \rightarrow \mathrm{C}_{1} \otimes \mathrm{C}_{2}
$$

is also fully faithful (indeed, interpret $\mathcal{C}_{1} \otimes-$ as $\operatorname{Funct}_{\text {cont }}\left(\mathcal{C}_{1}^{\vee},-\right)$ ).

Hence, the functors

$$
\operatorname{Shv\mathcal {N}}(y) \otimes \operatorname{Shv}_{\text {lisse }}(X) \rightarrow \operatorname{Shv}(y) \otimes \operatorname{Shv}_{\text {lisse }}(X) \rightarrow \operatorname{Shv}(y) \otimes \operatorname{Shv}(X)
$$

are both fully faithful. Combined with Theorem A.2.3 we obtain that the functor

$$
\operatorname{Shv}_{\mathcal{N}}(y) \otimes \operatorname{Shv}_{\text {lisse }}(X) \rightarrow \operatorname{Shv}_{\mathcal{N}^{\prime}}(y \times X)
$$

is also fully faithful. Hence, to prove Theorem A.3.9 it remains to prove that A.13 is essentially surjective.

A.4.5. From this point, the proof is essentially borrowed from [NY] Page 20].

According to $\underline{\mathrm{KS}}$, Corollary 8.3.22], we can choose a $\mu$-stratification of $y=\cup_{\alpha} y_{\alpha}$, such that $\mathcal{N}$ is contained in the union of the conormals to the strata. Consider the corresponding stratification $y_{\alpha} \times X$ of $y \times X$. This is still a $\mu$-stratification.

Let

$$
\operatorname{Shv}_{\text {str }}(y) \subset \operatorname{Shv}(y) \text { and } \operatorname{Shv}_{\operatorname{str}^{\prime}}(y \times X) \subset \operatorname{Shv}(y \times X)
$$

denote the full subcategories consisting of objects locally constant along the strata. By [KS] Proposition 8.4.1], we have

$$
\operatorname{Shv\mathcal {N}}(y) \subset \operatorname{Shv}_{\operatorname{str}}(y) \text { and } \operatorname{Shv}_{\mathcal{N}^{\prime}}(y \times X) \subset \operatorname{Shv}_{\operatorname{str}}(y \times X) .
$$

A.4.6. First, we claim that the functor

$$
\operatorname{Shv}_{\text {str }}(y) \otimes \operatorname{Shv}_{\text {lisse }}(X) \rightarrow \operatorname{Shv}_{\text {str }^{\prime}}(y \times X)
$$

is an equivalence.

By Sect. A.4.4 this functor is fully faithful. To prove that it is essentially surjective, by a Cousin argument, it reduces to the assertion that for each $\alpha$, the functor

$$
\operatorname{Shv}_{\text {lisse }}\left(y_{\alpha}\right) \otimes \operatorname{Shv}_{\text {lisse }}(X) \rightarrow \operatorname{Shvlisse}\left(y_{\alpha} \times X\right)
$$

is an equivalence.

However, this follows from Lemma A.4.2 and the fact that for any two $Y_{1}, Y_{2} \in \mathrm{Spc}$, the functor

$$
\mathrm{LS}\left(Y_{1}\right) \otimes \operatorname{LS}\left(Y_{2}\right) \rightarrow \operatorname{LS}\left(Y_{1} \times Y_{2}\right)
$$

is an equivalence (say, by Proposition 1.4.10).

A.4.7. Thus, it remains to show that if $\mathcal{F} \in \operatorname{Shv}_{\text {str }}(y) \otimes \operatorname{Shvlisse}_{(X)}$ is an object whose image along (A.14) belongs to $\operatorname{Shv}_{\mathcal{N}^{\prime}}(y \times X)$, then $\mathcal{F}$ itself belongs to $\operatorname{Shv\mathcal {N}}(y) \otimes \operatorname{Shv}_{\text {lisse }}(X)$.

With no restriction of generality, we can assume that $X$ is connected, and let us choose a base point $x \in X$. Interpreting $\operatorname{Shv}_{\text {lisse }}(X)$ via Lemma A.4.2 we obtain that if

$$
\mathrm{e}^{\prime} \hookrightarrow \mathcal{C}
$$

is a fully faithful map in DGCat, then

$$
\mathrm{e}^{\prime} \otimes \operatorname{Shv}_{\text {lisse }}(X) \rightarrow \mathcal{e} \otimes \operatorname{Shv}_{\text {lisse }}(X)
$$

is also fully faithful, and an object $c_{X} \in \mathcal{C} \otimes \operatorname{Shv}_{\text {lisse }}(X)$ belongs to $\mathcal{C}^{\prime} \otimes \operatorname{Shv}_{\text {lisse }}(X)$ if and only if its essential image under the evaluation functor

$$
\mathcal{C} \otimes \operatorname{Shv}_{\text {lisse }}(X) \stackrel{\operatorname{Id} \otimes \text { ev }_{x}}{\longrightarrow} \mathcal{C}
$$

belongs to $\mathrm{C}^{\prime}$. 
Consider the commutative diagram

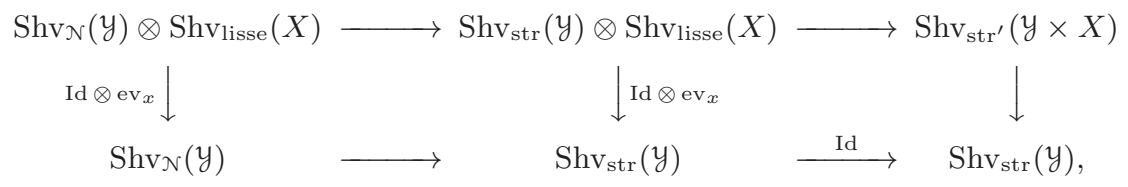

where the right vertical arrow is given by ${ }^{*}$-restriction along $\{x\} \hookrightarrow X$.

We obtain that it suffices to show that the functor

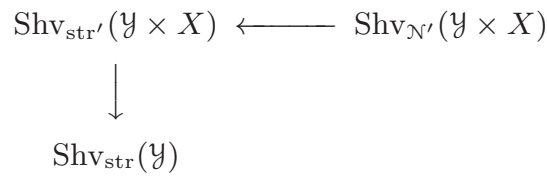

takes values in $\operatorname{Shv}_{\mathcal{N}}(y)$. This is a standard fact, but let us prove it for completeness.

A.4.8. Let $\mathcal{D}_{x}$ be an open disc around $x$. We have a commutative diagram

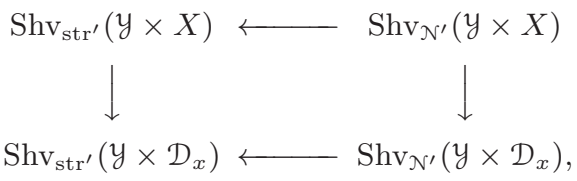

so it suffices to show that the functor

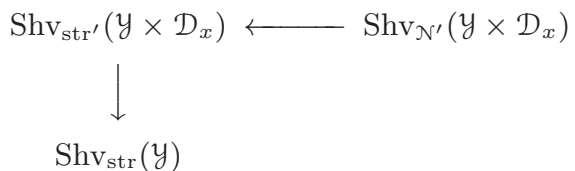

takes values in $\operatorname{Shv\mathcal {N}}(y)$.

However, by (A.14), the functor $\operatorname{Shv}_{\text {str }^{\prime}}\left(y \times \mathcal{D}_{x}\right) \rightarrow \operatorname{Shv}_{\text {str }}(y)$ is an equivalence, whose inverse is given by pullback. In particular, this inverse functor preserves singular support and hence defines an equivalence

$$
\operatorname{Shv\mathcal {N}}_{\mathcal{N}}(y) \rightarrow \operatorname{Shv}_{\mathcal{N}^{\prime}}\left(y \times \mathcal{D}_{x}\right)
$$

as desired.

\section{A.5. Proof of Theorem A.3.9 in the ind-constructible contexts.}

A.5.1. The proof of Theorem A.3.9 in case (a') follows verbatim the argument in case (a). Case (b") follows from case (a') by Lefschetz principle and Riemann-Hilbert.

We will now prove Theorem A.3.9 in cases (a') and (b')-(d') assuming that $X$ is proper.

With no restriction of generality, we can assume that $X$ is connected.

A.5.2. As in Sect. A.4.3 we reduce the assertion to the case when $y$ is an affine scheme.

By Proposition A.2.10 (e.g., using the argument in Sect. A.4.4), we know that the functor in question is fully faithful. Hence, it remains to show that it is essentially surjective.

Let $\mathcal{F}$ be an object in $\operatorname{Shv}_{\mathcal{N}^{\prime}}(y \times X)$. Since our functor preserves colimits, we can assume that $\mathcal{F}$ is constructible.

We will argue by Noetherian induction on $y$, so we will assume that the support of $\mathcal{F}$ is dominant over $y$ (otherwise, replace $y$ by the closure of the image of the support of $\mathcal{F}$ ). 
A.5.3. We claim that in the constructible case, $\mathcal{F}$ belongs to $\operatorname{Shv}_{\mathcal{N}^{\prime}}(y \times X)$ if and only if this holds for all of its perverse cohomology sheaves. Indeed:

-In case (a') this follows from the fact that SingSupp is measured by the functor of vanishing cycles, which is t-exact for the perverse t-structure.

-In cases (b'), (b") this follows from the definition.

-In cases (c), (d) and (d'), this follows from the definition of SingSupp in [Be] and the corresponding fact for the ULA property, see [Ga6].

For the same reason, for a short exact sequence of perverse sheaves

$$
0 \rightarrow \mathcal{F}_{1} \rightarrow \mathcal{F} \rightarrow \mathcal{F}_{2} \rightarrow 0
$$

we have

$$
\operatorname{SingSupp}(\mathcal{F})=\operatorname{SingSupp}\left(\mathcal{F}_{1}\right) \cup \operatorname{SingSupp}\left(\mathcal{F}_{2}\right)
$$

A.5.4. Hence, we can assume that $\mathcal{F}$ is of the form

$$
j_{! *}^{\prime}\left(\mathcal{F}_{U}\right)
$$

for a smooth locally closed

$$
\mathcal{U} \stackrel{j^{\prime}}{\rightarrow} y \times X
$$

and $\mathcal{F}_{\mathfrak{U}} \in \mathrm{Shv}_{\text {lisse }}(\mathcal{U})$ is perverse and lisse. With no restriction of generality we can assume that $\mathcal{U}$ is connected.

By the assumption in Sect. A.5.2, our $\mathcal{U}$ is dominant over $\mathcal{y}$. We claim that we can assume that $\mathcal{U}$ is of the form $\stackrel{0}{y} \times X$, where $\stackrel{\circ}{y} \stackrel{j}{\hookrightarrow} y$ is an open subset. This reduction will be carried out in Sects. A.5.5 A.5.6

A.5.5. By the transitivity property of $j_{! *}$, we can replace $\mathcal{U}$ by any of its non-empty open subsets. Let $y_{1} \subset y$ be a non-empty smooth open subset contained in the image of $\mathcal{U}$. Let $\mathcal{U}_{1}$ be the preimage of $y_{1}$ in $\mathcal{U}$.

We claim that $\mathcal{U}_{1}$ is dense in $y_{1} \times X$. Indeed, let $\bar{U}_{1}$ denote the closure of $\mathfrak{U}_{1}$ in $y_{1} \times X$. If $\mathfrak{U}_{1}$ were not dense in $y_{1} \times X$, the object $\mathcal{F} \mid y_{1} \times X$ would be the direct image under the closed embedding

$$
\bar{u}_{1} \hookrightarrow y_{1} \times X
$$

and hence $\operatorname{SingSupp}(\mathcal{F})$ would contain the conormals to $\bar{U}_{1}$ at each of its generic points. However, since $\mathcal{U}_{1} \rightarrow y_{1}$ is surjective, these conormals are not contained in

$$
T^{*}\left(y_{1}\right) \times\{\text { zero-section }\},
$$

contradicting the assumption on $\operatorname{SingSupp}(\mathcal{F})$.

A.5.6. Let $\stackrel{\circ}{\mathcal{U}}$ be an open subset

$$
\mathcal{U}_{1} \subseteq \stackrel{\circ}{\mathrm{U}} \subseteq y_{1} \times X
$$

maximal with respect to the property that the restriction to it of $\left.\mathcal{F}\right|_{y_{1} \times X}$ is lisse. We claim that $\stackrel{\circ}{\mathcal{U}}_{2}$ is all of $y_{1} \times X$. Once we prove this, we will be able to take $\stackrel{\circ}{y}:=y_{1}$, and thus achieve the reduction claimed in Sect. A.5.4

To prove the desired form of $\mathfrak{U}_{2}$ we argue as follows. By purity,

$$
D^{\prime}:=y_{1} \times X-\stackrel{\circ}{\mathcal{U}}
$$

is a divisor. We want to show that each irreducible component $D_{\alpha}^{\prime}$ of $D^{\prime}$ is the preimage of a divisor in $y_{1}$. Since $\stackrel{\circ}{\mathcal{U}} \rightarrow y_{1}$ is surjective, this would imply that $D^{\prime}$ is empty. 
Since $\left.\mathcal{F}\right|_{y_{1} \times X}$ is ramified around $D^{\prime}$, its singular support is not contained in the zero-section of $T^{*}\left(y_{1} \times X\right)$ near the generic point of each irreducible component $D_{\alpha}^{\prime}$ of $D^{\prime}$. Hence, for every such $D_{\alpha}^{\prime}$, there exists an irreducible component of $\mathcal{N}_{\alpha}^{\prime} \subset \operatorname{SingSupp}(\mathcal{F})$ such that the projection

$$
\mathcal{N}_{\alpha}^{\prime} \rightarrow T^{*}\left(y_{1} \times X\right) \rightarrow y_{1} \times X
$$

maps to $D_{\alpha}^{\prime}$ with positive-dimensional fibers.

We now use the assumption that

$$
\operatorname{SingSupp}(\mathcal{F}) \subset \mathcal{N} \times\{\text { zero-section }\} .
$$

We obtain that for each $\alpha$ there exists an irreducible component $\mathcal{N}_{\alpha}$ of $\mathcal{N}$ such that

$$
\mathcal{N}_{\alpha}^{\prime} \subset \mathcal{N}_{\alpha} \times X
$$

Let $D_{\alpha}$ be the (closure of the) image of $\mathcal{N}_{\alpha}$ along the map

$$
\mathcal{N}_{\alpha} \hookrightarrow T^{*}\left(y_{1}\right) \rightarrow y_{1} .
$$

We obtain a commutative diagram

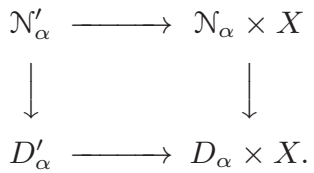

In particular, we have an inclusion

$$
D_{\alpha}^{\prime} \subseteq D_{\alpha} \times X
$$

Thus, it suffices to show that $D_{\alpha}$ is a divisor.

Suppose not. Then $D_{\alpha}$ would be all of $y_{1}$. Since $\mathcal{N}$ was assumed half-dimensional, we would obtain that $\mathcal{N}_{\alpha}$ is the zero-section. However, this would imply that $\mathcal{N}_{\alpha}^{\prime}$ is contained in the zero-section of $y_{1} \times X$. However, this contradicts the fact that the fibers of $\mathcal{N}_{\alpha}^{\prime} \rightarrow D_{\alpha}^{\prime}$ are positive-dimensional.

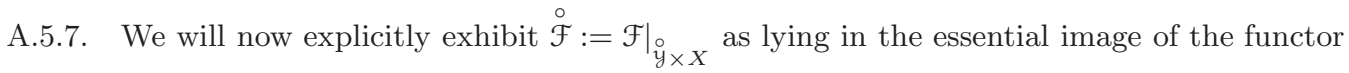

$$
\operatorname{Sh}_{\text {lisse }}(\stackrel{\circ}{y}) \otimes \operatorname{Shv}_{\text {lisse }}(X) \rightarrow \operatorname{Shv}_{\text {lisse }}(\stackrel{\circ}{y} \times X) .
$$

Pick a point $y \in \stackrel{\circ}{y}$ and let $\mathcal{F}_{X}$ be the !-restriction of $\stackrel{\circ}{\mathcal{F}}$ to $y \times X \subset y \times X$. Let $\pi_{y}$ and $\pi_{X}$ denote the projections from $y \times X$ to $y$ and $X$; let $\stackrel{\circ}{\pi}_{y}$ and $\stackrel{\circ}{\pi}_{X}$ denote their respective restrictions to $\stackrel{\circ}{y} \times X$.

Consider the object

$$
\stackrel{\circ}{\mathcal{F}}_{y}:=(\stackrel{\circ}{\pi})_{*}\left(\stackrel{\circ}{\mathcal{F}} \stackrel{!}{\otimes} \stackrel{\circ}{\pi}_{X}^{!}\left(\mathbb{D}\left(\mathcal{F}_{X}\right)\right)\right) \in \operatorname{Shv}(\stackrel{\circ}{y}) .
$$

Since $X$ is proper, $\stackrel{\circ}{\mathcal{F}} y$ is also lisse. The object $\stackrel{\circ}{\mathcal{F}} y$ is acted on the left by the associative algebra

$$
\mathcal{E} n d\left(\mathcal{F}_{X}^{\vee}\right) \simeq \mathcal{E} n d\left(\mathcal{F}_{X}\right)^{\mathrm{rev}} \text {. }
$$

By adjunction, we have a map

$$
\stackrel{\circ}{\pi} !\left(\stackrel{\circ}{\mathcal{F}}_{y}\right) \otimes \stackrel{\circ}{\pi}_{X}^{!}\left(\mathcal{F}_{X}\right) \simeq \stackrel{\circ}{\mathcal{F}}_{Y} \otimes \mathcal{F}_{X} \simeq \stackrel{\circ}{\pi}_{y}^{*}\left(\stackrel{\circ}{\mathcal{F}_{y}}\right) \otimes \stackrel{\circ}{\pi}_{X}^{*}\left(\mathcal{F}_{X}\right) \rightarrow \stackrel{\circ}{\mathcal{F}}
$$

Moreover, this map factors via a map

$$
\stackrel{\circ}{\mathcal{F}}_{Y} \underset{\mathcal{E} n d\left(\mathcal{F}_{X}\right)}{\otimes} \mathcal{F}_{X}:=\stackrel{\circ}{\pi} !\left(\stackrel{\circ}{\mathcal{F}_{y}}\right) \underset{\varepsilon n d\left(\mathcal{F}_{X}\right)}{\otimes} \stackrel{\circ}{\pi}_{X}^{\prime}\left(\mathcal{F}_{X}\right) \rightarrow \stackrel{\circ}{\mathcal{F}}
$$

We claim that A.18 is an isomorphism. Indeed, the !-fiber the map A.18) over y identifies with

$$
\mathrm{C}^{\bullet}\left(X, \mathcal{F}_{X} \stackrel{!}{\otimes} \mathbb{D}\left(\mathcal{F}_{X}\right)\right) \underset{\mathcal{E} n d\left(\mathcal{F}_{X}\right)}{\otimes} \mathcal{F}_{X} \simeq \mathcal{E} n d\left(\mathcal{F}_{X}\right) \underset{\mathcal{E} n d\left(\mathcal{F}_{X}\right)}{\otimes} \mathcal{F}_{X} \rightarrow \mathcal{F}_{X}
$$

and hence is an isomorphism (in the above formula $\mathrm{C}^{\bullet}(X,-)$ denotes the functor of global sections, i.e., sheaf cohomology at the cochain level). 
Since both sides in A.18 are lisse sheaves and $\stackrel{\circ}{y} \times X$ is connected, we obtain that A.18 is an isomorphism.

A.5.8. Set

$$
\mathcal{F}_{y}:=(\pi y)_{*}\left(\mathcal{F} \stackrel{!}{\otimes} \pi_{X}^{!}\left(\mathbb{D}\left(\mathcal{F}_{X}\right)\right)\right) \in \operatorname{Shv}(y) .
$$

Since singular support is preserved under direct images along proper maps, we have:

$$
\mathcal{F}_{y} \in \operatorname{Shv\mathcal {N}}(y) \text {. }
$$

Consider the object

$$
\mathcal{F}^{\prime}:=\mathcal{F}_{y} \underset{\mathcal{E} n d\left(\mathcal{F}_{X}\right)}{\bigotimes} \mathcal{F}_{X} \in \operatorname{Shv}_{\mathcal{N}^{\prime}}(y \times X):=\pi_{y}^{*}\left(\mathcal{F}_{y}\right) \underset{\mathcal{E} n d\left(\mathcal{F}_{X}\right)}{\otimes} \pi_{X}^{*}\left(\mathcal{F}_{X}\right) \in \operatorname{Shv}_{\mathcal{N}^{\prime}}(y \times X) .
$$

By construction, it belongs to the essential image of the functor

$$
\operatorname{Shv}_{\mathcal{N}}(y) \otimes \operatorname{Shv}_{\text {lisse }}(X) \rightarrow \operatorname{Shv}_{\mathcal{N}^{\prime}}(y \times X) .
$$

Moreover, by adjunction, we have a map

$$
\mathcal{F}^{\prime} \rightarrow \mathcal{F}
$$

which becomes an isomorphism when restricted to $\stackrel{\circ}{y} \times X$. Passing to the fiber of this map we accomplish the induction step (in our Noetherian induction on $y$ ).

\section{Appendix B. Spectral action in the context of Geometric Langlands (After [NY])}

In this section we will reprove a result from $\left[\mathrm{NY}\right.$ that says that the subcategory of $\operatorname{Shv}\left(\mathrm{Bun}_{G}\right)$ consisting of objects whose singular support belongs to the nilpotent cone carries a canonical action of $\operatorname{Rep}(\check{G})^{\otimes X^{\text {top,sing }}}$, where $X^{\text {top,sing }}$ is the object of Spc corresponding to $X$.

The proof we present will apply to any sheaf-theoretic context (see Theorem B.4.2).

\section{B.1. The players.}

B.1.1. For the duration of this section we let $X$ be a smooth projective curve over $k$. Let $G$ be a reductive group (over $k$ ).

Let $\operatorname{Bun}_{G}$ denote the moduli stack of $G$-bundles on $X$.

B.1.2. Recall that $T^{*}\left(\operatorname{Bun}_{G}\right)$ is the moduli space of pairs $(\mathcal{P}, \xi)$, where $\mathcal{P}$ is a $G$-bundle on $X$, and $\xi$ is an element of $\Gamma\left(X, \mathfrak{g}_{\mathcal{P}}^{*} \otimes \omega_{X}\right)$ where $\mathfrak{g}_{\mathcal{P}}^{*}$ is the vector bundle on $X$ associated to $\mathcal{P}$ and the co-adjoint representation $\mathfrak{g}^{*}$ of $G$.

Let

$$
\text { Nilp } \subset T^{*}\left(\operatorname{Bun}_{G}\right)
$$

be the nilpotent cone, defined to be the locus of pairs $(\mathcal{P}, \xi)$, where $\xi$ is nilpotent, i.e., maps to zero under the Chevalley map

$$
\mathfrak{g}^{*} \rightarrow \mathfrak{g}^{*} / / \operatorname{Ad}(G) \simeq \mathfrak{t}^{*} / / W
$$

B.1.3. Let $\operatorname{Shv}(-)$ be any of the sheaf-theoretic contexts from Sect. A.1.1 in which the ring e of coefficients is a field of characteristic 13 . Our interest in this section is the category $\operatorname{Shv}\left(\operatorname{Bun}_{G}\right)$ and its full subcategory

$$
\operatorname{Shv}_{\mathrm{Nilp}}\left(\operatorname{Bun}_{G}\right) \subset \operatorname{Shv}\left(\operatorname{Bun}_{G}\right) .
$$

Let $\check{G}$ be the Langlands dual of $G$, thought of as an algebraic group over e. We let Rep $(\check{G})$ denote the symmetric monoidal category of representations of $\check{G}$.

\footnotetext{
${ }^{13}$ The results of this section can be applied to any sheaf theoretic from Sect. A.1.1 but one will need to modify the category $\operatorname{Rep}(\breve{G})$ appropriately.
} 
B.2. The Hecke action. In this subsection we will discuss the general formalism of Hecke action.

Convention: for the duration of this subsection, when working in the sheaf-theoretic context (a), when we write DGCat we actually mean DGCat ${ }^{\text {discont}}$, and when we write $\otimes$, we actually mean $\stackrel{\text { discont }}{\otimes}$.

B.2.1. Let $A$ be an index category. Let

$$
a \mapsto \mathcal{M}_{a} \text { and } a \mapsto \mathcal{C}_{a}
$$

be functors

$$
\mathcal{M}_{A}: A \rightarrow \text { DGCat and } \mathcal{C}_{A}: A \rightarrow \text { DGCat }^{\text {Mon }} \text {, }
$$

respectively.

Then we can talk about an action of $\mathcal{C}_{A}$ on $\mathcal{M}_{A}$. Indeed, we can view $\mathcal{C}_{A}$ as an associative algebra object in the category Funct( $A$, DGCat), equipped with the level-wise (symmetric) monoidal structure.

Let $\operatorname{Act}\left(\mathcal{C}_{A}, \mathcal{M}_{A}\right)$ denote the space of such actions. In other words,

$$
\operatorname{Act}\left(\mathcal{C}_{A}, \mathcal{M}_{A}\right)=\operatorname{Maps}_{\mathbb{E}_{1}(\operatorname{Funct}(A, \text { DGCat }))}\left(\mathcal{C}_{A}, \underline{\operatorname{End}}\left(\mathcal{M}_{A}\right)\right) \text {. }
$$

Suppose now that we are given yet another functor

$$
\mathcal{C}_{A}^{\prime}: A \rightarrow \text { DGCat }^{\text {Mon }}, \quad a \mapsto \mathcal{C}_{a}^{\prime},
$$

equipped with a natural transformation

$$
\mathcal{C}_{A}^{\prime} \rightarrow \mathcal{C}_{A} .
$$

Given an action of $\mathcal{C}_{A}^{\prime}$ on $\mathcal{M}_{A}$ we can talk about an extension of this action to an action of $\mathcal{C}_{A}$. The space of such extensions is by definition

$$
\operatorname{Act}\left(\mathcal{C}_{A}, \mathcal{M}_{A}\right) \underset{\operatorname{Act}\left(\mathcal{C}_{A}^{\prime}, \mathcal{M}_{A}\right)}{\times}\{*\},
$$

where $\{*\} \rightarrow \operatorname{Act}\left(\mathfrak{C}_{A}^{\prime}, \mathcal{M}_{A}\right)$ is the initial action.

B.2.2. We take $A:=$ fSet. Take $\mathrm{C}_{A}^{\prime}$ to be the functor

$$
I \mapsto \operatorname{Shv}\left(X^{I}\right)
$$

where $\operatorname{Shv}\left(X^{I}\right)$ is viewed as a (symmetric) monoidal category with respect to the $\stackrel{!}{\otimes}$ operation.

Take $\mathcal{C}_{A}$ to be the functor

$$
I \mapsto \operatorname{Rep}(\check{G})^{\otimes I} \otimes \operatorname{Shv}\left(X^{I}\right)
$$

Take $\mathcal{M}_{A}$ to be the functor

$$
I \mapsto \operatorname{Shv}\left(\operatorname{Bun}_{G} \times X^{I}\right),
$$

equipped with a natural action of $\mathrm{C}_{A}^{\prime}$ (see Sect. B.2.5).

We will prove the following:

Proposition-Construction B.2.3. There exists a canonical extension of the action of (B.1) on (B.3) to an action of (B.2).

Remark B.2.4. It would follow from the construction, that in the sheaf-theoretic context (a), the restriction of the action in Proposition B.2.3 along

$$
\operatorname{Rep}(\breve{G})^{\otimes I} \otimes \operatorname{Shv}_{\text {lisse }}\left(X^{I}\right) \hookrightarrow \operatorname{Rep}(\breve{G})^{\otimes I} \otimes \operatorname{Shv}\left(X^{I}\right)
$$

is given by continuous functors, see Remark A.1.9

The rest of this subsection is devoted to the proof of Proposition B.2.3 It will be carried out using a certain formalism explained below. 
B.2.5. Let PreStk / Sch be the category of pairs

where $Z$ is a scheme, $y$ is prestack over $Z$.

Take $A=\operatorname{PreStk}^{\text {op }} / \mathrm{Sch}^{\mathrm{op}}$. Take $\mathcal{M}_{A}$ to be the functor

$$
(y, Z) \mapsto \operatorname{Shv}(y) .
$$

Take $\mathrm{C}_{A}^{\prime}$ to be the functor

$$
(y, Z) \mapsto \operatorname{Shv}(Z) .
$$

Then we have a natural action of $\mathcal{C}_{A}^{\prime}$ on $\mathcal{M}_{A}$, given by !-pullback along $y \rightarrow Z$.

B.2.6. Let Grpds / PreStk / Sch be the category of triples

$$
(\mathcal{H}, y, Z) \text {, }
$$

where $(y, Z)$ are as above, and where $\mathcal{H} \rightarrow \underset{Z}{\underset{Z}{x}} \underset{y}{ }$ is a groupoid acting on $y$ over $Z$, such that the projections $\mathcal{H} \rightrightarrows y$ are ind-schematic.

Note that $\operatorname{Shv}(\mathcal{H})$ is a monoidal category with respect to a convolution, defined to be the indextension of the $*$-convolution from compact objects.

Take $A=$ Grpds $^{\text {op }} /$ PreStk $^{\text {op }} / \mathrm{Sch}^{\text {op }}$. We take $\mathcal{M}_{A}$ to be the composition of the forgetful functor

$$
\text { Grpds / PreStk / Sch } \rightarrow \text { PreStk / Sch, } \quad(\mathcal{H}, y, Z) \mapsto(y, Z)
$$

and the functor B.4

We take $\mathcal{C}_{A}^{\prime}$ to be the composition of the forgetful functor (B.6) with (B.5).

We take $\mathcal{C}_{A}$ to be the functor that sends

$$
(\mathcal{H}, y, Z) \mapsto \operatorname{Shv}(\mathcal{H}) .
$$

Note that we have a canonical action of $\mathcal{C}_{A}$ on $\mathcal{M}_{A}$. We also have a canonical natural transformation

$$
\mathrm{C}_{A}^{\prime} \rightarrow \mathrm{C}_{A},
$$

given, level-wise, by the unit section of $\mathcal{H}$.

The induced action of $\mathcal{C}_{A}^{\prime}$ on $\mathcal{M}_{A}$ is one from Sect. B.2.5

Remark B.2.7. More formally, the above constructions should be spelled out as follows: we have the functors

that map Grpds / PreStk / Sch to

$$
(\mathcal{H}, y, Z) \mapsto y,(\mathcal{H}, y, Z) \mapsto Z,(\mathcal{H}, y, Z) \mapsto \mathcal{H}
$$

Corr(PreStk) $)_{\text {ind-sch,all, ComAlg(Corr(PreStk) }}$ ind-sch,all), AssocAlg(Corr(PreStk) $)_{\text {ind-sch,all }}$,

respectively, and

$$
\left(\mathcal{M}_{A}, \mathcal{C}_{A}^{\prime}, \mathcal{C}_{A}\right)
$$

are obtained by composing these functors with the functor $S_{\text {h }}$ Corr of Sect. A.1.7

B.2.8. We have a canonically defined functor

$$
\mathrm{fSet} \rightarrow \text { Grpds } / \text { PreStk } / \mathrm{Sch}, \quad I \mapsto \operatorname{Hecke}_{I} / \operatorname{Bun}_{G} \times X^{I} / X^{I},
$$

where Hecke $_{I}$ is the $I$-legged Hecke stack. (Note that Hecke $I$ and Bun ${ }_{G}$ are ordinary prestacks, so the construction of the functor (B.7) takes place in $(2,1)$-categories, i.e., involves finitely many pieces of data.)

Hence, in order to perform the construction in Proposition B.2.3, it suffices to construct a map (in the category Funct(fSet, DGCat $\left.{ }^{\text {Mon }}\right)$ ) from (B.2) to

$$
I \mapsto \operatorname{Shv}\left(\operatorname{Hecke}_{I}\right),
$$

extending the map from (B.1). 
B.2.9. Note, however, that in the context of Sect. B.2.6 the natural transformation

$$
(\mathcal{H}, y, Z) \mapsto(\operatorname{Shv}(Z) \rightarrow \operatorname{Shv}(\mathcal{H}))
$$

as functors Grpds / PreStk / Sch $\rightarrow$ DGCat $^{\text {Mon }}$, extends naturally to a natural transformation

$$
(\mathcal{H}, y, Z) \mapsto(\operatorname{Shv}(\mathcal{H}) \otimes \operatorname{Shv}(Z) \rightarrow \operatorname{Shv}(\mathcal{H})),
$$

expressing the fact that $\operatorname{Shv}(Z)$ maps to the center of $\operatorname{Shv}(\mathcal{H})$.

Hence, it suffices to construct a map from the functor

$$
I \mapsto \operatorname{Rep}(\check{G})^{\otimes I} .
$$

to $\mathrm{B} .8$.

B.2.10. The required natural transformation

$$
\operatorname{Rep}(\check{G})^{\otimes I} \rightarrow \operatorname{Shv}\left(\operatorname{Hecke}_{I}\right), \quad I \in \mathrm{fSet}
$$

is given by what is known as the naive geometric Satake functor. For completeness, we will recall its construction.

\section{B.3. Digression: naive geometric Satake.}

B.3.1. Recall that $S$-points of the Hecke stack Hecke ${ }_{I}$ are quadruples

$$
\left(x^{I}, \mathcal{P}^{\prime}, \mathcal{P}^{\prime \prime}, \alpha\right)
$$

where

$-x^{I}=\left\{x^{i}, i \in I\right\}$ is an $I$-tuple of $S$-points of $X$;

$-\mathcal{P}^{\prime}$ and $\mathcal{P}^{\prime \prime}$ are $G$-bundles on $S \times X$;

$-\alpha$ is an identification between $\mathcal{P}^{\prime}$ and $\mathcal{P}^{\prime \prime}$ on $S \times X-\cup_{i} \operatorname{Graph}_{x^{i}}$.

B.3.2. We introduce the local Hecke stack Hecke ${ }_{I}^{\text {loc }}$ as follows. Its $S$-points are quadruples

$$
\left(x^{I}, \mathcal{P}^{\prime}, \mathcal{P}^{\prime \prime}, \alpha\right)
$$

where:

$-x^{I}$ is an $I$-tuple of $S$-points of $X$;

$-\mathcal{P}^{\prime}$ and $\mathcal{P}^{\prime \prime}$ are $G$-bundles on $\mathcal{D}_{x^{I}}$-the parameterized formal disc around $x^{I}$ (i.e., the completion of the graph of $S \times X$ along $\left.\cup_{i} \operatorname{Graph}_{x^{i}}\right)$;

$-\alpha$ is an identification between $\mathcal{P}^{\prime}$ and $\mathcal{P}^{\prime \prime}$ on $\stackrel{\circ}{\mathcal{D}}_{x}$-the parameterized formal punctured disc around $x^{I}$ (see Ga4, Sect. 6.4.3]).

B.3.3. Convolution defines on $\operatorname{Shv}\left(\mathrm{Hecke}_{I}^{\mathrm{loc}}\right)$ a structure of monoidal category, and the assignment

$$
I \mapsto \operatorname{Shv}\left(\operatorname{Hecke}_{I}^{\text {loc }}\right)
$$

is a functor fSet $\rightarrow$ DGCat $^{\text {Mon }}$.

Restriction along $\mathcal{D}_{x^{I}} \rightarrow S \times X$ defines a map

$$
\mathfrak{r}_{I}: \operatorname{Hecke}_{I} \rightarrow \operatorname{Hecke}_{I}^{\text {loc }}
$$

The functors $\mathfrak{r}_{I}$ give rise to a natural transformation from (B.11) to (B.8). Thus, it is sufficient to construct a natural transformation

$$
\operatorname{Rep}(\check{G})^{\otimes I} \rightarrow \operatorname{Shv}\left(\operatorname{Hecke}_{I}^{\text {loc }}\right), \quad I \in \text { fSet } .
$$


Remark B.3.4. For the reader unwilling to consider the category Shv(Hecke ${ }_{I}^{\text {loc }}$ ) "as-is" (for reasons that the prestack Hecke ${ }_{I}^{\text {loc }}$ is not locally of finite type), it can be equivalently defined as

$$
\operatorname{Shv}\left(\operatorname{Gr}_{G, I}\right)^{\mathfrak{L}^{+}(G)_{I}}
$$

where

$-\mathrm{Gr}_{G, I}$ is the $I$-legged version of the affine Grassmannian;

$-\mathfrak{L}^{+}(G)_{I}$ is the group-scheme over $X^{I}$ of arcs into $G$.

B.3.5. Each of the categories $\operatorname{Rep}(\check{G})^{\otimes I}$ is endowed with a t-structure, for which the monoidal structure is t-exact. For a map $I_{1} \rightarrow I_{2}$ in fSet, the corresponding functor $\operatorname{Rep}(\breve{G})^{\otimes I_{1}} \rightarrow \operatorname{Rep}(\breve{G})^{\otimes I_{2}}$ is t-exact.

Each of the categories $\operatorname{Shv}\left(\mathrm{Hecke}_{I}^{\text {loc }}\right)$ is also endowed with a t-structure: this is the perverse tstructure shifted so that the dualizaing sheaf on the unit section

$$
X^{I} \rightarrow \operatorname{Hecke}_{I}^{\text {loc }}
$$

lies in the heart.

The monoidal operation on $\operatorname{Shv}\left(\operatorname{Hecke}_{I}^{\text {loc }}\right)$ is right t-exact, making $\left(\operatorname{Shv}\left(\operatorname{Hecke}_{I}^{\text {loc }}\right)\right)^{\ominus}$ into a monoidal abelian category. For a map $I_{1} \rightarrow I_{2}$ in fSet, the corresponding functor

$$
\operatorname{Shv}\left(\operatorname{Hecke}_{I_{1}}^{\text {loc }}\right) \rightarrow \operatorname{Shv}\left(\operatorname{Hecke}_{I_{2}}^{\text {loc }}\right)
$$

which is given by !-pullback, is right t-exact.

B.3.6. The starting point for the construction of the natural transformation (B.12) is a natural transformation

$$
\left(\operatorname{Rep}(\check{G})^{\otimes I}\right)^{\curvearrowright} \simeq \operatorname{Rep}\left(\check{G}^{I}\right)^{\curvearrowright} \rightarrow\left(\operatorname{Shv}\left(\operatorname{Hecke}_{I}^{\text {loc }}\right)\right)^{\curvearrowright}
$$

as functors fSet $\rightarrow \mathrm{AbCat}^{\mathrm{Mon}}$.

The natural transformation (B.13) is given by the geometric Satake functor of [MV].

Remark B.3.7. Although MV was stated in the context of (a'), it applies in any (ind)-constructible context. The construction in cases (a) and (b) follows from that in cases (a') and (b'), respectively.

B.3.8. Let us now explain how to use (B.13) to obtain the desired natural transformation (B.10).

The first key property of $(\mathrm{B} .13$ is that for each $I \in$ fSet, the corresponding functor is t-exact. Hence, B.13 gives rise to a system of t-exact functors

$$
\left(\operatorname{Rep}(\check{G})^{\otimes I}\right)^{b} \simeq D\left(\left(\operatorname{Rep}(\check{G})^{\otimes I}\right)^{\oslash}\right)^{b} \rightarrow \operatorname{Shv}\left(\text { Hecke }_{I}^{\text {loc }}\right),
$$

equipped with a right-lax monoidal structure (here $D(-)^{b}$ stands for the bounded derived category of a given abelian category, equipped with its natural DG structure, see [Lu2, Sect. 1.3.3]; we are using its universal property, which is a variant of [Lu2, Theorem 1.3.3.2] for the bounded derived category).

The second key property of (B.13) is that the right-lax monoidal structure on (B.14) is strict. Hence, precomposing with

$$
\left(\operatorname{Rep}(\check{G})^{\otimes I}\right)^{c} \hookrightarrow\left(\operatorname{Rep}(\check{G})^{\otimes I}\right)^{b}
$$

and ind-extending, we obtain the desired natural transformation (B.12).

\section{B.4. Hecke action on the subcategory with nilpotent singular support.}


B.4.1. Let Nilp $\subset T^{*}\left(\operatorname{Bun}_{G}\right)$ be the nilpotent cone. Consider the full subcategory

$$
\operatorname{Shv}_{N i l p}\left(\operatorname{Bun}_{G}\right) \subset \operatorname{Shv}\left(\operatorname{Bun}_{G}\right) \text {. }
$$

Our goal is to prove the following:

Theorem-Construction B.4.2. There exists a natural transformation between the following two functors

from the functor

$$
\text { fSet } \rightarrow \text { DGCat }^{\text {Mon }}:
$$

to the functor

$$
I \mapsto \operatorname{Rep}(\check{G})^{\otimes I}
$$

$$
I \mapsto \operatorname{End}\left(\operatorname{Shv}_{\text {Nilp }}\left(\operatorname{Bun}_{G}\right)\right) \otimes \operatorname{Shvlisse}\left(X^{I}\right) .
$$

B.4.3. Before we prove Theorem B.4.2 let us explain how it recovers the result of $\mathrm{NY}$.

Let us specialize to the sheaf-theoretic context (a) from Sect. A.1.1

Recall (see Lemma A.4.2) that in this case, we have a functorial identification

$$
\operatorname{Shv}_{\text {lisse }}\left(X^{I}\right) \simeq \operatorname{LS}\left(\left(X^{\text {top,sing }}\right)^{I}\right) .
$$

Hence, combining with Sect. 1.7.3 we obtain:

Corollary B.4.4. We have a canonically defined action of $\operatorname{Rep}(\check{G})^{\otimes X^{\mathrm{top}, \operatorname{sing}}}$ on $\operatorname{Shv}_{\mathrm{Nilp}}\left(\operatorname{Bun}_{G}\right)$.

Finally, combining with Theorem 1.5.5 we obtain:

Corollary B.4.5. There is a canonically defined action of the (symmetric) monoidal category $\left.\mathrm{QCoh}_{\left(\operatorname{LocSys}_{\bar{G}}\right.}\left(X^{\text {top,sing }}\right)\right)$ on $\operatorname{Shv}_{\mathrm{Nil}_{\mathrm{p}}}\left(\operatorname{Bun}_{G}\right)$.

B.4.6. Note that by Lemma A.4.2 the stack $\operatorname{LocSys}_{\breve{G}}\left(X^{\text {top,sing }}\right)$ that appears in Corollary B.4.5 identifies canonically with the Betti version of the stack of $\breve{G}$-local systems on $X$.

\section{B.5. Proof of Theorem B.4.2.}

B.5.1. Consider the full subcategory

$$
\operatorname{Shv}_{\mathrm{Nilp}^{\prime}}\left(\operatorname{Bun}_{G} \times X^{I}\right) \subset \operatorname{Shv}\left(\operatorname{Bun}_{G} \times X^{I}\right),
$$

where

$$
\text { Nilp }^{\prime}:=\operatorname{Nilp} \times\{\text { zero-section }\} \subset T^{*}\left(\operatorname{Bun}_{G} \times X^{I}\right) .
$$

The crucial ingredient for the proof is the following geometric assertion:

Theorem B.5.2 ([NY). For every $I \in \mathrm{fSet}$, the action of $\operatorname{Rep}(\check{G})^{\otimes I}$ on $\operatorname{Shv}\left(\operatorname{Bun}_{G} \times X^{I}\right)$ preserves the full subcategory

$$
\operatorname{Shv}_{\mathrm{Nilp}^{\prime}}\left(\operatorname{Bun}_{G} \times X^{I}\right) \subset \operatorname{Shv}\left(\operatorname{Bun}_{G} \times X^{I}\right) .
$$

We will prove Theorem B.5.2 in Sect. B.6 below.

Let us show how Theorem B.5.2 leads to the construction of the natural transformation in Theorem B.4.2

B.5.3. Let us return to the situation of Sect. B.2.1 Let us be given an action of $\mathcal{C}_{A}$ on $\mathcal{M}_{A}$, and let

$$
\mathcal{M}_{A}^{\prime}: A \rightarrow \text { DGCat, } \quad a \mapsto \mathcal{M}_{a}^{\prime}
$$

be another functor. Let $\mathcal{M}_{A}^{\prime}$ be equipped with a natural transformation to $\mathcal{M}_{A}$, such that for every $a \in A$, the corresponding functor

is fully faithful.

$$
\mathcal{M}_{a}^{\prime} \rightarrow \mathcal{M}_{a}
$$

Assume being given an action of $\mathcal{C}_{A}$ on $\mathcal{M}_{A}$ so that for every $a \in A$, the action of $\mathcal{C}_{a}$ on $\mathcal{M}_{a}$ preserves the subcategory $\mathcal{M}_{a}^{\prime}$. In this case we obtain an action of $\mathcal{C}_{A}$ on $\mathcal{M}_{A}^{\prime}$. 
B.5.4. Let us take $A=\mathrm{fSet}$, and $\mathrm{C}_{A}$ be the functor

$$
I \mapsto \operatorname{Rep}(\check{G})^{\otimes I} \otimes \operatorname{Shv}_{\text {lisse }}\left(X^{I}\right) .
$$

We take $\mathcal{M}_{A}$ to be the functor $\mathrm{B.3}$. We let $\mathcal{C}_{A}$ act on $\mathcal{M}_{A}$ by precomposing the action of Proposition B.2.3 with the natural transformation

$$
\operatorname{Rep}(\check{G})^{\otimes I} \otimes \operatorname{Shv}_{\text {lisse }}\left(X^{I}\right) \rightarrow \operatorname{Rep}(\check{G})^{\otimes I} \otimes \operatorname{Shv}\left(X^{I}\right), \quad I \in \text { fSet } .
$$

We take $\mathcal{M}_{A}^{\prime}$ to be the family of full subcategories

$$
\operatorname{Shv}_{\mathrm{Nilp}^{\prime}}\left(\operatorname{Bun}_{G} \times X^{I}\right) \subset \operatorname{Shv}\left(\operatorname{Bun}_{G} \times X^{I}\right), \quad I \in \mathrm{fSet} .
$$

Thus, by Sect. B.5.3 and Theorem B.5.2 we obtain an action of (B.15) on

$$
I \mapsto \operatorname{Shv}_{\mathrm{Nilp}^{\prime}}\left(\operatorname{Bun}_{G} \times X^{I}\right)
$$

B.5.5. Let us be again in the situation of Sect. B.2.1 and let us be given an action of $\mathcal{C}_{A}$ on $\mathcal{M}_{A}$. Assume now that $\mathcal{C}_{A}$ arises as a tensor product

$$
\mathcal{C}_{A}^{1} \otimes \mathcal{C}_{A}^{2},
$$

Assume also that $\mathcal{M}_{A}$ arises as as

$$
\mathcal{M}^{1} \otimes \mathcal{C}_{A}^{2}
$$

for some fixed DG category $\mathcal{M}^{1}$.

Consider the action of $\mathcal{C}_{A}^{2}$ on $\mathcal{M}$ given by right multiplication along the second factor. Then the datum of extension of this action of $\mathcal{C}_{A}^{2}$ to an action of $\mathcal{C}_{A}$ is equivalent to that of a natural transformation

$$
\mathcal{C}_{A}^{1} \rightarrow \operatorname{End}\left(\mathcal{M}^{1}\right) \otimes\left(\mathcal{C}_{A}^{2}\right)^{\mathrm{rev}}
$$

of functors $A \rightarrow$ DGCat $^{\text {Mon }}$.

B.5.6. We apply this to $\mathcal{C}_{A}^{1}$ being the functor $(\mathrm{B.9})$ and $\mathcal{C}_{A}^{2}$ being the functor

$$
I \mapsto \operatorname{Shv}_{\text {lisse }}\left(X^{I}\right) .
$$

Take $\mathcal{M}^{1}=\operatorname{Shv}_{\text {Nilp }}\left(\operatorname{Bun}_{G}\right)$. Now, by Theorem A.3.9 the natural transformation

$$
\operatorname{Shv}_{\text {Nilp }}\left(\operatorname{Bun}_{G}\right) \otimes \operatorname{Shv}_{\text {lisse }}\left(X^{I}\right) \rightarrow \operatorname{Shv}_{\mathrm{Nilp}^{\prime}}\left(\operatorname{Bun}_{G} \times X^{I}\right)
$$

is an isomorphism.

Hence, by Sect. B.5.5, the action of (B.15) on (B.16) gives rise to a sought-for natural transformation

$$
\operatorname{Rep}(\check{G})^{\otimes I} \rightarrow \operatorname{End}\left(\operatorname{Shv}_{\text {Nilp }}\left(\operatorname{Bun}_{G}\right)\right) \otimes \operatorname{Shv}_{\text {lisse }}\left(X^{I}\right), \quad I \in \text { fSet . }
$$

$\square[$ Theorem B.4.2

B.6. Preservation of nilpotence of singular support. The goal of this subsection is to prove Theorem B.5.2. The proof is essentially a paraphrase of [NY].

B.6.1. By Theorem A.3.9 we have to show that for $\mathcal{F} \in \operatorname{Shv}_{\text {Nilp }}\left(\operatorname{Bun}_{G}\right)$ and any $V \in \operatorname{Rep}(\check{G})^{\otimes I}$, we have

$$
\mathrm{H}(V, \mathcal{F}) \in \operatorname{Shv}_{\mathrm{Nilp}^{\prime}}\left(\operatorname{Bun}_{G} \times X^{I}\right) \subset \operatorname{Shv}\left(\operatorname{Bun}_{G} \times X^{I}\right),
$$

where $H(-,-)$ denotes the Hecke action

$$
\operatorname{Rep}(\check{G})^{\otimes I} \otimes \operatorname{Shv}\left(\operatorname{Bun}_{G}\right) \rightarrow \operatorname{Shv}\left(\operatorname{Bun}_{G} \times X^{I}\right) .
$$

By the associativity of the Hecke action, we can assume that $I=\{*\}$. 
B.6.2. Let Hecke ${ }_{X}$ denote the 1-legged global Hecke stack. Let Heckeloc denote the 1-legged local Hecke stack, defined in Sect. B.3.2 Let $\mathfrak{r}$ denote the restriction map

$$
\operatorname{Hecke}_{X} \rightarrow \operatorname{Hecke}_{X}^{\text {loc }} \text {. }
$$

We have the diagram

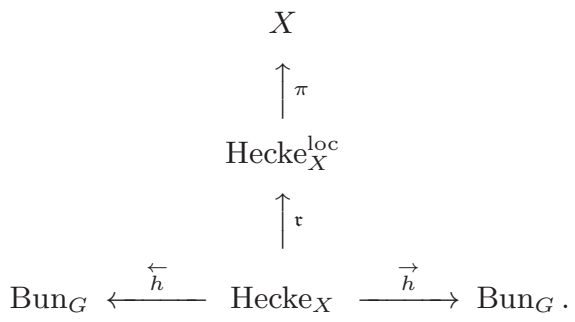

Denote also

$$
\mathfrak{s}:=\pi \circ \mathfrak{r}: \operatorname{Hecke}_{X} \rightarrow X .
$$

With these notations, we have:

$$
\mathrm{H}(V, \mathcal{F})=(\vec{h} \times \mathfrak{s})_{*} \circ(\overleftarrow{h} \times \mathfrak{r})^{!}\left(\mathcal{F} \otimes \mathcal{S}_{V}\right)
$$

where $\mathcal{S}_{V} \in \operatorname{Shv}\left(\right.$ Hecke $\left._{X}^{\text {loc }}\right)$ corresponds to $V \in \operatorname{Rep}(\check{G})$ by geometric Satake.

B.6.3. Note that the map

$$
\overleftarrow{h} \times \mathfrak{s}: \operatorname{Hecke}_{X} \rightarrow \operatorname{Bun}_{G} \times \operatorname{Hecke}_{X}^{\text {loc }}
$$

is (pro)-smooth, while the map

$$
\vec{h} \times \mathfrak{s}: \operatorname{Hecke}_{X} \rightarrow \operatorname{Bun}_{G} \times X
$$

is (ind)-proper.

For a given point $\left(x, \mathcal{P}^{\prime}, \mathcal{P}^{\prime \prime}, \alpha\right) \in$ Hecke $_{X}$ consider the corresponding diagram of cotangent spaces

$$
\begin{aligned}
& \begin{array}{l}
T_{x}^{*}(X) \\
\downarrow \downarrow(d \pi)^{*}
\end{array} \\
& \begin{array}{c}
T_{x}^{*}(X) \\
\downarrow \downarrow(d \pi)^{*}
\end{array}
\end{aligned}
$$

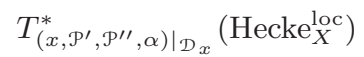

$$
\begin{aligned}
& \downarrow(d \mathfrak{r})^{*} \\
& T_{\mathcal{P}}^{*}\left(\operatorname{Bun}_{G}\right) \stackrel{(d \overleftarrow{h})^{*}}{\longrightarrow} \quad T_{\left(x, \mathcal{P}^{\prime}, \mathcal{P}^{\prime \prime}, \alpha\right)}^{*}\left(\operatorname{Hecke}_{X}\right) \stackrel{(d \vec{h})^{*}}{\longleftarrow} T_{\mathcal{P}^{\prime \prime}}^{*}\left(\operatorname{Bun}_{G}\right)
\end{aligned}
$$

Hence, in order to prove (B.17), it suffices to show the following. Consider a quadruple of elements

$$
\xi^{\prime} \in T_{\mathcal{P}^{\prime}}^{*}\left(\operatorname{Bun}_{G}\right), \xi^{\prime \prime} \in T_{\mathcal{P}^{\prime \prime}}^{*}\left(\operatorname{Bun}_{G}\right), \xi_{H} \in T_{\left.\left(x, \mathcal{P}^{\prime}, \mathcal{P}^{\prime \prime}, \alpha\right)\right|_{\mathcal{D}_{x}}}\left(\operatorname{Hecke}_{X}^{\text {loc }}\right), \xi_{X} \in T_{x}^{*}(X),
$$

so that

- $\xi^{\prime}$ is nilpotent;

- $\xi_{H} \in \operatorname{SingSupp}\left(\mathcal{S}_{V}\right)$;

- $(d \overleftarrow{h})^{*}\left(\xi^{\prime}\right)+(d \mathfrak{r})^{*}\left(\xi_{H}\right)=(d \vec{h})^{*}\left(\xi^{\prime \prime}\right)+(d \mathfrak{s})^{*}\left(\xi_{X}\right)$

We need to prove that in this case:

- $\xi^{\prime \prime}$ is also nilpotent;

- $\xi_{X}=0$. 
Indeed, once we show this, B.17) would follow by combining the following two assertions:

-For a smooth map $f: y_{1} \rightarrow y_{2}$ and $\mathcal{F} \in \operatorname{Shv}\left(y_{2}\right)$, the subset $\operatorname{SingSupp}\left(f^{!}(\mathcal{F})\right) \subset T^{*}\left(y_{1}\right)$ equals the image of

$$
\operatorname{SingSupp}(\mathcal{F}) \underset{y_{2}}{\times} y_{1} \subset T^{*}\left(y_{2}\right) \underset{y_{2}}{\times} y_{1}
$$

along the codifferential map

$$
T^{*}\left(y_{2}\right) \underset{y_{2}}{\times} y_{1} \rightarrow T^{*}\left(y_{1}\right)
$$

-For a proper map $f: y_{1} \rightarrow y_{2}$ and $\mathcal{F} \in \operatorname{Shv}\left(y_{1}\right)$, the $\operatorname{subset} \operatorname{SingSupp}\left(f_{*}(\mathcal{F})\right) \subset T^{*}\left(y_{2}\right)$ is contained in the image along the projection

$$
T^{*}\left(y_{2}\right) \underset{y_{2}}{\times} y_{1} \rightarrow T^{*}\left(y_{2}\right)
$$

of the preimage of $\operatorname{SingSupp}(\mathcal{F})$ along (B.20).

B.6.4. Let $\operatorname{Hecke}_{x}$ (resp., Hecke $e_{x}^{\text {loc }}$ ) be the fiber of Hecke $X$ (resp., Hecke ${ }_{X}^{\text {loc }}$ ) over $x$. Along with the diagrams (B.18) and B.19) consider the diagrams

$\operatorname{Hecke}_{x}^{\text {loc }}$

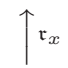

and

$$
\operatorname{Bun}_{G} \stackrel{\overleftarrow{h}_{x}}{\longleftarrow} \operatorname{Hecke}_{x} \stackrel{\vec{h}_{x}}{\longrightarrow} \operatorname{Bun}_{G}
$$

$$
\begin{gathered}
T_{\left.\left(\mathcal{P}^{\prime}, \mathcal{P}^{\prime \prime}, \alpha\right)\right|_{\mathcal{D} x}}^{*}\left(\text { Hecke }_{x}^{\text {loc }}\right) \\
\downarrow\left(d \mathfrak{r}_{x}\right)^{*} \\
T_{\mathcal{P}^{\prime}}^{*}\left(\operatorname{Bun}_{G}\right) \stackrel{\left(d \overleftarrow{h}_{x}\right)^{*}}{\longrightarrow} T_{\left(\mathcal{P}^{\prime}, \mathcal{P}^{\prime \prime}, \alpha\right)}^{*}\left(\operatorname{Hecke}_{x}\right) \stackrel{\left(d \vec{h}_{x}\right)^{*}}{\longleftarrow} T_{\mathcal{P}^{\prime \prime}}^{*}\left(\operatorname{Bun}_{G}\right) .
\end{gathered}
$$

Let $\xi_{H_{x}}$ denote the image of $\xi_{H}$ under the restriction map

$$
T_{\left(x, \mathcal{P}^{\prime}, \mathcal{P}^{\prime \prime}, \alpha\right)}^{*}\left(\text { Hecke }_{X}^{\text {loc }}\right) \rightarrow T_{\left(\mathcal{P}^{\prime}, \mathcal{P}^{\prime \prime}, \alpha\right)}^{*}\left(\text { Hecke }_{x}^{\text {loc }}\right) .
$$

We have a commutative diagram

$$
\begin{gathered}
T_{\left.\left(\mathcal{P}^{\prime}, \mathcal{P}^{\prime \prime}, \alpha\right)\right|_{\mathcal{D}_{x}}\left(\operatorname{Hecke}_{x}^{\text {loc }}\right) \stackrel{\left(d \mathfrak{r}_{x}\right)^{*}}{\longrightarrow}} T_{\left(\mathcal{P}^{\prime}, \mathcal{P}^{\prime \prime}, \alpha\right)}^{*}\left(\text { Hecke }_{x}\right) \\
T_{\left.\left(x, \mathcal{P}^{\prime}, \mathcal{P}^{\prime \prime}, \alpha\right)\right|_{\mathcal{D}_{x}}}^{*}\left(\text { Hecke }_{X}^{\text {loc }}\right) \stackrel{(d \mathfrak{r})^{*}}{\longrightarrow} T_{\left(x, \mathcal{P}^{\prime}, \mathcal{P}^{\prime \prime}, \alpha\right)}^{*}\left(\text { Hecke }_{X}\right) .
\end{gathered}
$$

Hence, the assumption on $\left(\xi^{\prime}, \xi^{\prime \prime}, \xi_{H}\right)$ implies

$$
\left(d \overleftarrow{h}_{x}\right)^{*}\left(\xi^{\prime}\right)+\left(d \mathfrak{r}_{x}\right)^{*}\left(\xi_{H_{x}}\right)=\left(d \vec{h}_{x}\right)^{*}\left(\xi^{\prime \prime}\right)
$$

B.6.5. We identify

$$
T_{\mathcal{P}^{\prime}}^{*}\left(\operatorname{Bun}_{G}\right):=\Gamma\left(X, \mathfrak{g}_{\mathcal{P}^{\prime}}^{*} \otimes \omega_{X}\right) \text { and } T_{\mathcal{P}^{\prime \prime}}^{*}\left(\operatorname{Bun}_{G}\right):=\Gamma\left(X, \mathfrak{g}_{\mathcal{P}^{\prime \prime}}^{*} \otimes \omega_{X}\right)
$$

Further, we identify $T_{\left.\left(\mathcal{P}^{\prime}, \mathcal{P}^{\prime \prime}, \alpha\right)\right|_{\mathcal{D}_{x}} ^{*}}^{*}\left(\operatorname{Hecke}_{x}\right)$ with the dual of $R^{1} \Gamma(X, \mathrm{~K})$ where $\mathrm{K}$ is the complex

$$
\mathrm{K}:=\text { Cone }\left(\mathfrak{g}_{\mathcal{P}^{\prime}} \oplus \mathfrak{g}_{\mathcal{P}^{\prime \prime}} \rightarrow j_{*}\left(j^{*}\left(\mathfrak{g}_{\mathcal{P}^{\prime}}\right) \stackrel{\alpha}{\simeq} j^{*}\left(\mathfrak{g}_{\mathcal{P}^{\prime \prime}}\right)\right)\right),
$$

where $j$ denotes the embedding $X-x \hookrightarrow X$.

Finally, we identify $T_{\left.\left(\mathcal{P}^{\prime}, \mathcal{P}^{\prime \prime}, \alpha\right)\right|_{\mathcal{D}_{x}} ^{*}}\left(\right.$ Hecke $\left._{x}^{\text {loc }}\right)$ with the dual of $R^{1} \Gamma\left(\mathcal{D}_{x}, \mathrm{~K}\right)$ where $\mathcal{D}_{x}$ (resp., $\left.\stackrel{\circ}{\mathcal{D}}_{x}\right)$ is the formal (resp., formal punctured disc) around $x$ (see Sect. B.3.2). 
Since $\mathcal{D}_{x}$ is affine, the above $R^{1} \Gamma\left(\mathcal{D}_{x}, \mathrm{~K}\right)$ identifies with

$$
\Gamma\left(\mathcal{D}_{x}, H^{1}(\mathrm{~K})\right)
$$

which is the same as $\Gamma\left(X, H^{1}(\mathrm{~K})\right)$, since $H^{1}(\mathrm{~K})$ is set-theoretically supported at $x \in X$.

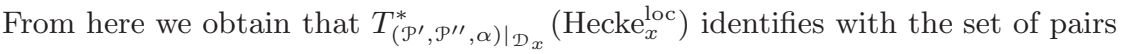

$$
\left(\xi_{\text {loc }}^{\prime} \in \Gamma\left(\mathcal{D}_{x}, \mathfrak{g}_{\mathcal{P}^{\prime}}^{*} \otimes \omega_{X}\right), \xi_{\text {loc }}^{\prime \prime} \in \Gamma\left(\mathcal{D}_{x}, \mathfrak{g}_{\mathcal{P}^{\prime \prime}}^{*} \otimes \omega_{X}\right)\right),
$$

such that

as elements of $\Gamma\left(\stackrel{\circ}{\mathcal{D}}_{x}, \mathfrak{g}_{\mathcal{P}^{\prime \prime}}^{*} \otimes \omega_{X}\right)$.

$$
\alpha\left(\xi_{\text {loc }}^{\prime}\right)=\xi_{\text {loc }}^{\prime \prime}
$$

B.6.6. Equation (B.21) translates into

$$
\alpha\left(\left.\xi^{\prime}\right|_{\mathcal{D}_{x}}\right)=\left.\xi^{\prime \prime}\right|_{\mathcal{D}_{x}}
$$

In particular, we obtain:

$$
\xi^{\prime} \text { is nilpotent }\left.\Rightarrow \xi^{\prime}\right|_{\mathcal{D}_{x}} \text { is nilpotent }\left.\Rightarrow \xi^{\prime \prime}\right|_{\mathcal{D}_{x}} \text { is nilpotent } \Rightarrow \xi^{\prime \prime} \text { is nilpotent. }
$$

Thus, it remains to show that $\xi_{X}=0$.

B.6.7. Note that the short exact sequences

$$
\begin{aligned}
& 0 \rightarrow T_{x}^{*}(X) \rightarrow T_{\left(x, \mathcal{P}^{\prime}, \mathcal{P}^{\prime \prime}, \alpha\right)}^{*}\left(\operatorname{Hecke}_{X}\right) \rightarrow T_{\left(\mathcal{P}^{\prime}, \mathcal{P}^{\prime \prime}, \alpha\right)}^{* \text { Hecke }) \rightarrow 0} \\
& 0 \rightarrow T_{x}^{*}(X) \rightarrow T_{\left.\left(x, \mathcal{P}^{\prime}, \mathcal{P}^{\prime \prime}, \alpha\right)\right|_{\mathcal{D}_{x}}}^{*}\left(\text { Hecke }_{X}^{\text {loc }}\right) \rightarrow T_{\left.\left(\mathcal{P}^{\prime}, \mathcal{P}^{\prime \prime}, \alpha\right)\right|_{\mathcal{D}_{x}}}^{*}\left(\text { Hecke }_{x}^{\text {loc }}\right) \rightarrow 0
\end{aligned}
$$

admit a canonical splittings (compatible with the map between these short exact sequences induced by $\mathfrak{r})$ :

These splittings are a consequence of the fact that the prestacks

$$
\text { Hecke }_{X} \text { and Hecke } \text { loc }_{X}
$$

each carries a canonical structure of crystal along $X$. Indeed, for a test-scheme $S$ and two points

$$
x_{1}, x_{2}: S \rightrightarrows X
$$

such that that

$$
\left.x_{1}\right|_{S_{\text {red }}}=\left.x_{2}\right|_{S_{\text {red }}}
$$

the data of lifting of these points to points of Hecke $_{X}$ (resp., Hecke ${ }_{X}^{\text {loc }}$ ) coincide. This is because (B.26) implies

$$
S \times X-\mathrm{Graph}_{x_{1}}=S \times X-\mathrm{Graph}_{x_{2}} \text { and } \mathcal{D}_{x_{1}}=\mathcal{D}_{x_{2}}, \stackrel{\circ}{\mathcal{D}}_{x_{1}}=\stackrel{\circ}{\mathcal{D}}_{x_{2}} .
$$

B.6.8. Consider the resulting maps

$$
T_{\left(x, \mathcal{P}^{\prime}, \mathcal{P}^{\prime \prime}, \alpha\right)}^{*}\left(\operatorname{Hecke}_{X}\right) \rightarrow T_{x}^{*}(X)
$$

and

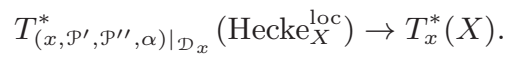

It follows from the definition of the above crystal structure that the composition of (B.27) with the maps

vanishes.

$$
T_{\mathcal{P}^{\prime}}^{*}\left(\operatorname{Bun}_{G}\right) \stackrel{(d \overleftarrow{h})^{*}}{\longrightarrow} T_{\left(x, \mathcal{P}^{\prime}, \mathcal{P}^{\prime \prime}, \alpha\right)}^{*}\left(\operatorname{Hecke}_{X}\right) \stackrel{(d \vec{h})^{*}}{\longleftarrow} T_{\mathcal{P}^{\prime \prime}}^{*}\left(\operatorname{Bun}_{G}\right)
$$

Therefore, to prove that $\xi_{X}=0$, it suffices to show the following. Let $\xi_{H} \in T_{\left.\left(x, \mathcal{P}^{\prime}, \mathcal{P}^{\prime \prime}, \alpha\right)\right|_{\mathcal{D}_{x}}}\left(\operatorname{Hecke}_{X}^{\text {loc }}\right)$ be a vector that belongs to $\operatorname{SingSupp}\left(\mathcal{S}_{V}\right)$. Suppose that

$$
\xi_{H_{x}} \in T_{\left.\left(\mathcal{P}^{\prime}, \mathcal{P}^{\prime \prime}, \alpha\right)\right|_{\mathcal{D}_{x}}}\left(\mathrm{Hecke}_{x}^{\text {loc }}\right)
$$

is nilpotent, i.e., the corresponding pair $\left(\xi_{\text {loc }}^{\prime}, \xi_{\text {loc }}^{\prime \prime}\right)$ in $\underline{B .23}$ consists of nilpotent elements. 
Then we need to prove that the image of $\xi_{H}$ along $\mathrm{B.28}$ is zero.

B.6.9. To prove the latter assertion, we can assume that $X=\mathbb{A}^{1}$ and $x=0$. In this case, we identify

$$
\text { Hecke }_{\mathbb{A}^{1}}^{\text {loc }} \simeq \operatorname{Hecke}_{0}^{\text {loc }} \times \mathbb{A}^{1} .
$$

With respect to (B.29), the object $\mathcal{S}_{V}$ is the pullback along the projection

$$
\text { Hecke }_{\mathbb{A}^{1}}^{\text {loc }} \rightarrow \text { Hecke }_{0}^{\text {loc }} \text {. }
$$

In addition, from (B.29), we obtain a different splitting of B.25). The discrepancy between the two different splittings of (B.25) is a map

$$
T_{\left(\mathcal{P}^{\prime}, \mathfrak{P}^{\prime \prime}, \alpha\right)}^{*}\left(\text { Hecke }_{0}^{\text {loc }}\right) \rightarrow T_{0}^{*}\left(\mathbb{A}^{1}\right) \simeq k .
$$

It suffices to show that this map vanishes on nilpotent elements (we refer to the description of $T_{\left(\mathcal{P}^{\prime}, \mathfrak{P}^{\prime \prime}, \alpha\right)}^{*}\left(\mathrm{Hecke}_{x}^{\text {loc }}\right)$ given by (B.23); when we say "nilpotent", we mean that the corresponding elements $\xi_{\text {loc }}^{\prime}$ and $\xi_{\text {loc }}^{\prime \prime}$ are nilpotent).

B.6.10. Let us calculate the map (B.30). Let $\mathfrak{L}(G)_{X}$ be the version of the loop group spread over $X$, and let $\mathfrak{L}(G)_{x}$ be its fiber at $x \in X$. The group ind-scheme $\mathfrak{L}(G)_{X}$ also has a crystal structure along $X$, which gives rise to a splitting of the short exact sequence

$$
0 \rightarrow T_{g}\left(\mathfrak{L}(G)_{x}\right) \rightarrow T_{g}\left(\mathfrak{L}(G)_{X}\right) \rightarrow T_{x}(X) \rightarrow 0, \quad g \in \mathfrak{L}(G)_{x} .
$$

For $X=\mathbb{A}^{1}$, we have an identification

$$
\mathfrak{L}(G)_{\mathbb{A}^{1}} \simeq \mathfrak{L}(G)_{0} \times \mathbb{A}^{1},
$$

and hence a different splitting of the short exact sequence (B.31). The discrepancy between these two splittings is a map

$$
k \simeq T_{0}\left(\mathbb{A}^{1}\right) \rightarrow T_{g}\left(\mathfrak{L}(G)_{0}\right) .
$$

Let $u$ denote the coordinate on $\mathbb{A}^{1}$. We will identify the tangent space $T_{g}\left(\mathfrak{L}(G)_{0}\right)$ with $\mathfrak{g}((u))$ by means of the left translation by $g$. Under this identification the map (B.32), thought of as an element of $\mathfrak{g}((u))$ is

$$
g^{-1} \cdot \frac{d g}{d u}
$$

i.e., the (left) logarithmic derivative of $g$ with respect to the coordinate $u$.

B.6.11. Let $\left(\mathcal{P}^{\prime}, \mathcal{P}^{\prime \prime}, \alpha\right)$ be a point of

$$
\operatorname{Hecke}_{0}^{\text {loc }} \simeq \mathfrak{L}(G)_{0} \backslash \mathfrak{L}(G) / \mathfrak{L}(G)_{0},
$$

represented by an element $g \in \mathfrak{L}(G)_{0}$. By the Cartan decomposition, we can assume that $g=u^{\lambda}$ for a dominant coweight $\lambda$.

Note that the corresponding element

$$
\lambda \cdot u^{-1} \subset \mathfrak{t}((u)) \subset \mathfrak{g}((u))
$$

We need to show that for a nilpotent element

$$
\xi \in T_{\left(x, \mathcal{P}^{\prime}, \mathfrak{P}^{\prime \prime}, \alpha\right)}^{*}\left(\operatorname{Hecke}_{0}^{\text {loc }}\right) \simeq \mathfrak{g}^{*} \llbracket u \rrbracket d u \cap \operatorname{Ad}_{u^{\lambda}}\left(\mathfrak{g}^{*} \llbracket u \rrbracket d u\right) \subset \mathfrak{g}^{*}((u)) d u,
$$

the residue pairing

against $\lambda \cdot u^{-1}$ evaluates to 0 .

$$
\mathfrak{g}((u)) \times \mathfrak{g}^{*}((u)) d u \rightarrow k
$$

Set

$$
\xi_{0}:=\xi \bmod u \in \mathfrak{g}^{*} d u \simeq \mathfrak{g}^{*} .
$$

This is a nilpotent element of $\mathfrak{g}^{*}$. We need to show that its pairing with $\lambda \in \mathfrak{t} \subset \mathfrak{g}$ gives zero. 
B.6.12. Let

$$
\mathfrak{g}=\mathfrak{n}_{\lambda}^{+} \oplus \mathfrak{m}_{\lambda} \oplus \mathfrak{n}_{\lambda}^{-}
$$

be the triangular decomposition of $\mathfrak{g}$ corresponding to $\lambda$, i.e., $\mathfrak{m}_{\lambda}$ is the centralizer of $\lambda$ and $\mathfrak{n}_{\lambda}^{+}$(resp., $\mathfrak{n}_{\lambda}^{-}$) corresponds to positive (resp., negative) eigenvalues of $\lambda$ for the adjoint action.

Consider the corresponding decomposition

$$
\xi_{0}=\xi_{0}^{+}+\xi_{0}^{0}+\xi_{0}^{-}
$$

of $\xi_{0}$.

Note, however, that the condition that $\xi \in \operatorname{Ad}_{u^{\lambda}}\left(\mathfrak{g}^{*} \llbracket u \rrbracket d u\right)$ implies that $\xi_{0}^{-}$is zero. Knowing that, the fact that $\xi_{0}$ is nilpotent implies that $\xi_{0}^{0}$ is a nilpotent element of $\mathfrak{m}_{\lambda}$.

We have

$$
\left\langle\lambda, \xi_{0}\right\rangle=\left\langle\lambda, \xi_{0}^{+}\right\rangle+\left\langle\lambda, \xi_{0}^{0}\right\rangle=\left\langle\lambda, \xi_{0}^{0}\right\rangle .
$$

However, the latter is zero, being the pairing of a central element (in the reductive Lie algebra $\mathfrak{m}_{\lambda}$ ) with a nilpotent one.

\section{Appendix C. Integrated actions in the context of D-Modules}

In Proposition 1.7.2 we described what it takes to have an action of $\mathcal{A}^{\otimes Y}$ on a DG category $\mathcal{M}$ for $Y \in$ Spc. In this section we will use the RHS of Proposition 1.7 .2 to give a definition of integrated action in the context of D-modules.

We will then study how our definition plays out in the context of geometric Langlands.

\section{C.1. Definition of integrated action.}

C.1.1. Let us place ourselves in the sheaf-theoretic context (b) from Sect. A.1.1 In this case our ring of coefficients e is the same as the ground field $k$. Thus, we will work with $k$-linear DG categories.

C.1.2. Let $X$ be a scheme over $k$ (in the applications to geometric Langlands, $X$ will be a smooth projective curve).

For a symmetric monoidal DG category $\mathcal{A}$ and a DG category $\mathcal{M}$, an action of $\mathcal{A}^{\otimes X}$ on $\mathcal{M}$ is by definition a natural transformation between the following two functors fSet $\rightarrow$ DGCat $^{\text {mon }}$ :

From the functor

$$
I \mapsto \mathcal{A}^{\otimes I},
$$

to the functor

$$
I \mapsto \operatorname{End}(\mathcal{M}) \otimes \mathrm{D}-\bmod \left(X^{I}\right) .
$$

The totality of categories equipped with an action of $\mathcal{A}^{\otimes X}$ forms a 2-category, which we will denote

$$
\mathcal{A}^{\otimes X}-\bmod .
$$

Remark C.1.3. It is easy to see that the datum of action of $\mathcal{A}^{\otimes X}$ on $\mathcal{M}$ is equivalent to that of action on $\mathcal{M}$ of the (non-unital) symmetric monoidal category $\operatorname{Fact}(\mathcal{A})_{\operatorname{Ran}(X)}$, defined as in Ga3, Sect. 2.5].

C.1.4. Example. Let $X$ be a curve and let $G$ be a reductive group.

Take $\mathcal{A}=\operatorname{Rep}(\check{G})$ and $\mathcal{M}=\mathrm{D}-\bmod \left(\operatorname{Bun}_{G}\right)$. Then Proposition B.2.3 says that we have an action of $\operatorname{Rep}(\check{G})^{\otimes X}$ on D-mod($\left(\operatorname{Bun}_{G}\right)$. 
C.1.5. Let us be given an action of $\mathcal{A}^{\otimes X}$ on $\mathcal{M}$. For a finite set $I$ and $r \in \mathcal{A}^{\otimes I}$, let $\mathcal{S}_{I}(r)$ denote the resulting functor

$$
\mathcal{M} \rightarrow \mathcal{M} \otimes \mathrm{D}-\bmod \left(X^{I}\right) .
$$

Lemma C.1.6. Let $r$ be dualizable. Then the functor $\mathcal{S}_{I}(r)$ admits a continuous right adjoint.

Proof. The adjoint in question is explicitly given by

$$
\begin{aligned}
\mathcal{M} \otimes \mathrm{D}-\bmod \left(X^{I}\right) \stackrel{\mathcal{S}_{I}\left(r^{\vee}\right) \otimes \operatorname{Id}_{\mathrm{D}-\bmod \left(X^{I}\right)}}{\longrightarrow} \mathcal{M} \otimes \mathrm{D}-\bmod \left(X^{I}\right) \otimes \mathrm{D}-\bmod \left(X^{I}\right) \stackrel{\operatorname{Id}_{\mathcal{M}} \otimes \Delta^{!}}{\longrightarrow} & \\
& \rightarrow \mathcal{M} \otimes \mathrm{D}-\bmod \left(X^{I}\right) \stackrel{\mathrm{Id}_{\mathcal{M}} \otimes \Gamma_{\mathrm{dR}}\left(X^{I},-\right)}{\longrightarrow} \mathcal{M} .
\end{aligned}
$$

C.1.7. The ULA property. Let us assume that $\mathcal{A}$ is compactly generated and rigid, and that $\mathcal{M}$ is compactly generated.

For a pair of compact objects $r \in \mathcal{A}^{\otimes I}$ and $m \in \mathcal{M}$ consider again the object

$$
\mathcal{S}_{I}(r)(m) \in \mathcal{M} \otimes \mathrm{D}-\bmod \left(X^{I}\right)
$$

Note that by Lemma C.1.6 this object is automatically compact. We claim:

Proposition C.1.8. The object $\mathcal{S}_{I}(r)(m)$ is $U L A$ over $X^{I}$.

We refer the reader to Sect. D] where the notion of ULA is reviewed.

Proof. Note that the dual of $\mathcal{M} \otimes \mathrm{D}-\bmod \left(X^{I}\right)$ as a $\mathrm{D}-\bmod \left(X^{I}\right)$-module category identifies with

$$
\mathcal{M}^{\vee} \otimes \operatorname{D}-\bmod \left(X^{I}\right) .
$$

Let $m^{\vee} \in \mathcal{M}^{\vee}$ be the abstract Verdier dual of $m \in \mathcal{M}$. Let $r^{\vee}$ be the monoidal dual of $r$. Then the object

$$
\mathcal{S}_{I}\left(r^{\vee}\right)\left(m^{\vee}\right) \in \mathcal{M}^{\vee} \otimes \mathrm{D}-\bmod \left(X^{I}\right)
$$

satisfies the requirements from Sect. D.1.4.

\section{C.2. Lisse actions.}

C.2.1. Note that for a smooth scheme $Y$, the functor

$$
\mathrm{D}-\bmod _{\text {lisse }}(Y) \rightarrow \mathrm{D}-\bmod (Y)
$$

is a fully faithful embedding that admits a continuous right adjoint.

Hence, for another DG category $\mathcal{M}$, the functor

$$
\mathcal{M} \otimes \mathrm{D}-\bmod _{\text {lisse }}(Y) \rightarrow \mathcal{M} \otimes \mathrm{D}-\bmod (Y)
$$

is also fully faithful and admits a continuous right adjoint given by tensoring the right adjoint to (C.3) with $\operatorname{Id}_{\mathcal{M}}$.

Further, if $\mathcal{M} \rightarrow \mathcal{M}^{\prime}$ is conservative, an object of $\mathcal{M} \otimes \mathrm{D}-\bmod (Y)$ belongs to $\mathcal{M} \otimes \mathrm{D}$-mod $\operatorname{misse}_{\text {lise }}(Y)$ if and only if its image in $\mathcal{M}^{\prime} \otimes \mathrm{D}-\bmod (Y)$ belongs to $\mathcal{M}^{\prime} \otimes \mathrm{D}-\bmod _{\text {lisse }}(Y)$. 
C.2.2. For a pair of objects $r \in \mathcal{A}^{\otimes I}$ and $m \in \mathcal{M}$ consider the object

$$
\mathcal{S}_{I}(r)(m) \in \mathcal{M} \otimes \mathrm{D}-\bmod \left(X^{I}\right)
$$

We shall say that the action of $\mathcal{A}^{\otimes X}$ on $\mathcal{M}$ is lisse if for every $r$ and $m$ as above, the object (C.4) belongs to the full subcategory.

$$
\mathcal{M} \otimes \mathrm{D}-\bmod _{\text {lisse }}\left(X^{I}\right) \subset \mathcal{M} \otimes \mathrm{D}-\bmod \left(X^{I}\right) .
$$

Note also that by associativity, it is enough to check this condition for $I=\{*\}$.

Let

$$
\mathcal{A}^{\otimes X}-\bmod ^{\text {lisse }} \subset \mathcal{A}^{\otimes X}-\bmod
$$

denote the corresponding full subcategory.

C.2.3. Example. The statement of Theorem B.5.2 is equivalent to the assertion that the action of $\operatorname{Rep}(\check{G})^{\otimes X}$ on D-mod $\operatorname{Nilp}_{\text {p }}\left(\operatorname{Bun}_{G}\right)$ is lisse.

C.2.4. Given an action of $\mathcal{A}^{\otimes X}$ on $\mathcal{M}$, let

$$
\mathcal{M}^{\text {lisse }} \subset \mathcal{M}
$$

be the full subcategory consisting of objects $m$, for which C.4 with $I=\{*\}$ belongs to the subcategory $\mathcal{M} \otimes \mathrm{D}$-mod $\operatorname{misse}(X)$ for all $r$.

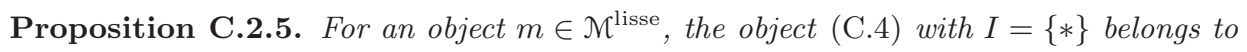

$$
\mathcal{M}^{\text {lisse }} \otimes \mathrm{D}-\bmod _{\text {lisse }}(X) \subset \mathcal{M} \otimes \mathrm{D}-\bmod _{\text {lisse }}(X) .
$$

Proof. By the last remark in Sect. C.2.1 it suffices to show that the functors $\mathcal{S}_{\{*\}}(r)$ send $\mathcal{M}^{\text {lisse }}$ to $\mathcal{M}^{\text {lisse }} \otimes \mathrm{D}-\bmod (X)$.

For any DG category $\mathcal{C}$, consider the full subcategory

$$
(\mathcal{M} \otimes \mathcal{C})^{\text {lisse }} \subset \mathcal{M} \otimes \mathcal{C} .
$$

I.e., we apply the definition of $(-)^{\text {lisse }}$ to $\mathcal{M} \otimes \mathcal{C}$ on which $\mathcal{A}^{\otimes X}$ acts via the first factor.

Explicitly, this subcategory consists of objects $m^{\prime}$ for which

$$
\left(\mathcal{S}_{\{*\}}\left(r^{\prime}\right) \otimes \operatorname{Id}_{\mathcal{C}}\right)\left(m^{\prime}\right) \in \mathcal{M} \otimes \mathrm{D}-\bmod _{\text {lisse }}(X) \otimes \mathcal{C} \subset \mathcal{M} \otimes \mathrm{D}-\bmod (X) \otimes \mathcal{C} \text { for all } r^{\prime} \in \mathcal{A} .
$$

The functor

factors via a functor

$$
\mathcal{M}^{\text {lisse }} \otimes \mathcal{C} \rightarrow \mathcal{M} \otimes \mathcal{C}
$$

$$
\mathcal{M}^{\text {lisse }} \otimes \mathcal{C} \rightarrow(\mathcal{M} \otimes \mathcal{C})^{\text {lisse }} .
$$

We claim that if $\mathcal{C}$ is dualizable, then $\mathrm{C.5}$ is an equivalence. Indeed, this follows by interpreting $\mathcal{M} \otimes \mathcal{C}$ as

$$
\text { Funct }_{\text {cont }}\left(\varrho^{\vee}, \mathcal{M}\right) \text {. }
$$

Hence, applying this to $\mathrm{C}=\mathrm{D}$ - $\bmod (X)$, it suffices to show that for any $r$ and $r^{\prime}$, we have

$$
\left(\mathcal{S}_{\{*\}}\left(r^{\prime}\right) \otimes \operatorname{Id}_{\mathcal{C}}\right) \circ \mathcal{S}_{\{*\}}(r)(m) \in \mathcal{M} \otimes \mathrm{D}-\bmod _{\text {lisse }}(X) \otimes \mathrm{D}-\bmod (X) .
$$

We have

$$
\left(\mathcal{S}_{\{*\}}\left(r^{\prime}\right) \otimes \operatorname{Ide}\right) \circ \mathcal{S}_{\{*\}}(r)(m)=\mathcal{S}_{\{*\} \sqcup\{*\}}\left(r^{\prime} \otimes r\right)(m) \simeq \sigma\left(\mathcal{S}_{\{*\} \sqcup\{*\}}\left(r \otimes r^{\prime}\right)(m)\right),
$$

where $\sigma$ denotes the transposition acting on $X \times X$.

Hence, it suffices to show that

$$
\mathcal{S}_{\{*\} \sqcup\{*\}}\left(r \otimes r^{\prime}\right)(m) \subset \mathcal{M} \otimes \mathrm{D}-\bmod (X) \otimes \mathrm{D}-\bmod _{\text {lisse }}(X) .
$$

However,

$$
\mathcal{S}_{\{*\} \sqcup\{*\}}\left(r \otimes r^{\prime}\right)(m) \simeq\left(\mathcal{S}_{\{*\}}(r) \otimes \operatorname{Id}_{\mathcal{C}}\right) \circ \mathcal{S}_{\{*\}}\left(r^{\prime}\right)(m)
$$


and the assertion follows from the fact that

$$
\mathcal{S}_{\{*\}}\left(r^{\prime}\right)(m) \subset \mathcal{M} \otimes \mathrm{D}-\bmod _{\text {lisse }}(X),
$$

by the assumption on $m$.

Corollary C.2.6. The subcategory $\mathcal{M}^{\text {lisse }}$ carries an action of $\mathcal{A}^{\otimes X}$, and this action is lisse.

C.2.7. Thus, Theorem B.5.2 says that we have an inclusion:

$$
\mathrm{D}-\bmod _{\mathrm{Nil}}\left(\operatorname{Bun}_{G}\right) \subset\left(\mathrm{D}-\bmod \left(\operatorname{Bun}_{G}\right)\right)^{\text {lisse }}
$$

We propose the following:

Conjecture C.2.8. The inclusion C.6 is an equality.

C.2.9. Example. Let us call an object $\mathcal{F} \in \mathrm{D}-\bmod \left(\operatorname{Bun}_{G}\right)$ a loose Hecke eigensheaf, if for every $V \in$ $\operatorname{Rep}(\check{G})$ we have an isomorphism

$$
H(V, \mathcal{F}) \simeq \mathcal{F}^{\prime} \otimes E_{V},
$$

where $E_{V}$ is some object of $\mathrm{D}-\bmod _{\text {lisse }}(X)$ and $\mathcal{F}^{\prime} \in \mathrm{D}-\bmod \left(\operatorname{Bun}_{G}\right)$.

Clearly, a loose Hecke eigensheaf belongs to $\left(\mathrm{D}-\bmod \left(\mathrm{Bun}_{G}\right)\right)^{\text {lisse }}$. Hence, Conjecture C.2.8 contains as a special case the following conjecture, first proposed by G. Laumon (for actual Hecke eigensheaves, rather than loose ones):

Conjecture C.2.10. A loose Hecke eigensheaf has a nilpotent singular support.

C.3. A spectral characterization of lisse actions. In this subsection we specialize to the case when $\mathcal{A}=\operatorname{Rep}(\mathrm{G})$ for an algebraic group $\mathrm{G}$, and $X$ is a smooth and proper curve.

C.3.1. Let $\operatorname{LocSys}_{\mathrm{G}}(X)$ be the stack of de Rham G-local systems on $X$, defined as in AG, Sect. 10.1].

It is easy to see that one can write $\operatorname{LocSys}_{\mathrm{G}}(X)$ as a quotient of a quasi-compact (derived) scheme by an action of the algebraic group. From this it follows that $\mathrm{QCoh}\left(\operatorname{LocSys}_{\mathrm{G}}(X)\right)$ is compactly generated and rigid.

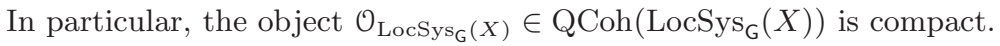

C.3.2. Recall (see Ga1, Sect. 4.3]) that there exists a canonically defined symmetric monoidal functor

$$
\operatorname{Fact}(\operatorname{Rep}(\mathrm{G}))_{\operatorname{Ran}(X)} \rightarrow \mathrm{QCoh}\left(\operatorname{LocSys}_{\mathrm{G}}(X)\right),
$$

which admits a continuous and fully faithful right adjoint.

The functor (C.7) defines a fully faithful embedding

$$
\mathrm{QCoh}\left(\operatorname{LocSys}_{\mathrm{G}}(X)\right)-\bmod \rightarrow \mathcal{A}^{\otimes X}-\bmod .
$$

C.3.3. Let $\mathcal{M}$ be a compactly generated category equipped with an action of $\mathrm{QCoh}\left(\operatorname{LocSys}_{\mathrm{G}}(X)\right)$.

To a compact object $m \in \mathcal{M}$ one can attach its set-theoretic support

$$
\operatorname{supp}(m) \subset \operatorname{LocSys}_{\mathrm{G}}(X) \text {. }
$$

This is the smallest among closed subsets $Y \subset \operatorname{LocSys}_{\mathrm{G}}(X)$ such that the image of $m$ under the functor

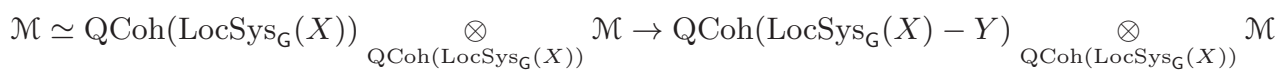

is zero. 
C.3.4. Let $\mathrm{P}$ be a parabolic in $\mathrm{G}$ with Levi quotient $\mathrm{M}$, and consider the diagram

$$
\operatorname{LocSys}_{\mathrm{G}}(X) \stackrel{\mathrm{p}_{\mathrm{P}}}{\longleftarrow} \operatorname{LocSys}_{\mathrm{P}}(X) \stackrel{\mathrm{qP}_{\mathrm{P}}}{\longrightarrow} \operatorname{LocSys}_{\mathrm{M}}(X) \text {. }
$$

Let $\sigma$ be an irreducible M-local system on $X$. Note that we have a closed embedding

$$
i_{\sigma}: \mathrm{pt} / \operatorname{Aut}(\sigma) \hookrightarrow \operatorname{LocSys}_{\mathrm{M}}(X) \text {. }
$$

In the above formula, $\operatorname{Aut}(\sigma)$ is the group of automorphisms of $\sigma$. It contains the $Z(\mathrm{M})$ (the center of $\mathrm{M})$, and by the irreducibility assumption, $\operatorname{Aut}(\sigma) / \mathrm{M}$ is finite.

Denote

$$
\operatorname{LocSys}_{\mathrm{P}, \sigma}(X):=\operatorname{LocSys}_{\mathrm{P}}(X) \underset{\operatorname{LocSys}_{\mathrm{M}}(X)}{\times} \operatorname{pt} / \operatorname{Aut}(\sigma) .
$$

Let $\mathrm{p}_{\mathrm{P}, \sigma}$ denote the projection

$$
\operatorname{LocSys}_{\mathrm{P}, \sigma}(X) \rightarrow \operatorname{LocSys}_{\mathrm{G}}(X) .
$$

Let

$$
Y_{\mathrm{P}, \sigma} \subset \operatorname{LocSys}_{\mathrm{G}}(X)
$$

be a closed subset equal to the image of $\mathrm{p}_{\mathrm{P}, \sigma}$.

Proposition C.3.5. Let $m \in \mathcal{M}$ be a compact object such that $\operatorname{supp}(m)$ is contained in some $Y_{\mathrm{P}, \sigma}$. Then $m \in \mathcal{M}^{\text {lisse }}$.

Proof. It is enough to prove the proposition for

$$
\mathcal{M}:=\mathrm{QCoh}\left(\operatorname{LocSys}_{\mathrm{G}}(X)\right)_{Y_{\mathrm{P}, \sigma}},
$$

where the latter is the full subcategory of $\mathrm{QCoh}\left(\operatorname{LocSys}_{\mathrm{G}}(X)\right)$ consisting of objects with set-theoretic support on $Y_{\mathrm{P}, \sigma}$.

Note that the functor

$$
\mathrm{p}_{\mathrm{P}, \sigma}^{*}: \mathrm{QCoh}\left(\operatorname{LocSys}_{\mathrm{G}}(X)\right)_{Y_{\mathrm{P}, \sigma}} \rightarrow \mathrm{QCoh}_{\left(\operatorname{LocSys}_{\mathrm{P}, \sigma}(X)\right)}
$$

is conservative. Hence, by the last remark in Sect. C.2.1 we can further replace the category

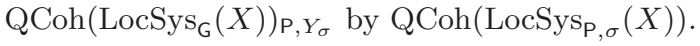

The action of $\operatorname{Rep}(\mathrm{G})^{\otimes X}$ on $\mathrm{QCoh}\left(\operatorname{LocSys}_{\mathrm{P}, \sigma}(X)\right)$ factors through the restriction

$$
\operatorname{Rep}(\mathrm{G})^{\otimes X} \rightarrow \operatorname{Rep}(\mathrm{P})^{\otimes X} \text {. }
$$

Hence, it is enough to show that the action of $\operatorname{Rep}(\mathrm{P})^{\otimes X}$ on $\mathrm{QCoh}\left(\operatorname{LocSys}_{\mathrm{P}, \sigma}(X)\right)$ is lisse.

Next, we note that the essential image of the restriction functor

$$
\operatorname{Rep}(\mathrm{M}) \rightarrow \operatorname{Rep}(\mathrm{P})
$$

generates $\operatorname{Rep}(\mathrm{P})$. Hence, it is enough to show that the action of $\operatorname{Rep}(\mathrm{M})^{\otimes X}$ on $\mathrm{QCoh}\left(\operatorname{LocSys}_{\mathrm{P}, \sigma}(X)\right)$ is lisse.

Next, we note that the map

$$
\mathrm{qP}, \sigma: \operatorname{LocSys}_{\mathrm{P}, \sigma}(X) \rightarrow \mathrm{pt} / \operatorname{Aut}(\sigma)
$$

has the property that its base change with respect to $\mathrm{pt} \rightarrow \mathrm{pt} / \operatorname{Aut}(\sigma)$ yields an algebraic stack of the form

derived affine scheme $/ \mathbb{A}^{n}$

for some $n$. Hence, the direct image functor

$$
\mathrm{QCoh}\left(\operatorname{LocSys}_{\mathrm{P}, \sigma}(X)\right) \rightarrow \mathrm{QCoh}(\mathrm{pt} / \operatorname{Aut}(\sigma))
$$

is conservative. Hence, it is enough to show that the action of $\operatorname{Rep}(\mathrm{M})^{\otimes X}$ on $\mathrm{QCoh}(\mathrm{pt} / \operatorname{Aut}(\sigma))$ is lisse.

Finally, applying the (conservative) pullback functor along pt $\rightarrow \mathrm{pt} / \operatorname{Aut}(\sigma)$, we obtain that it is enough to show that the action of $\operatorname{Rep}(\mathrm{M})^{\otimes X}$ on Vect, corresponding to the M-local system $\sigma$, is lisse. 
However, the latter is evident: for $V \in \operatorname{Rep}(\mathrm{M})^{c}$ and $k \in$ Vect, the corresponding object

$$
\mathcal{S}_{\{*\}}(V)(k) \in \operatorname{Vect} \otimes \mathrm{D}-\bmod (X) \simeq \mathrm{D}-\bmod (X)
$$

is just $V_{\sigma}$, the twist of $V$ by $\sigma$.

C.3.6. The next conjecture proposes to describe the subcategory $\mathcal{M}^{\text {lisse }} \subset \mathcal{M}$ in terms of set-theoretic support:

Conjecture C.3.7. A compact object $m \in \mathcal{M}$ belongs to $\mathcal{M}^{\text {lisse }}$ if and only if it can be written as a colimit of objects $m_{\alpha}$, for each of which $\operatorname{supp}\left(m_{\alpha}\right)$ is contained in a finite union of closed subsets of the form $Y_{\mathrm{P}, \sigma}$.

\section{Appendix D. The notion of universal local ACyClicity (ULA)}

In this section we review the notion of universal local acyclicity, for a general sheaf-theoretic context with the exclusion of (a). In the particular case of D-modules we will express it via the forgetful functor to quasi-coherent sheaves.

\section{D.1. The abstract ULA property.}

D.1.1. In this subsection $\mathcal{C}$ will be an abstract symmetric monoidal DG category. The example to keep in mind is $\mathcal{C}=\mathrm{D}-\bmod (Y)$ for a scheme $Y$ with respect to $\stackrel{!}{\otimes}$, so $\mathcal{C}$ is very far from being rigid.

D.1.2. Let $\mathcal{M}$ be a $\mathcal{C}$-module category, which is dualizable as a $\mathcal{C}$-module. This means that there exists another $\mathcal{C}$-module category $\mathcal{M}^{\vee}, \mathrm{e}$ and functors

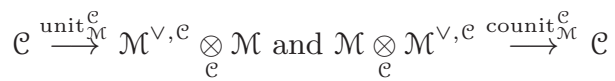

that satisfy the usual duality axioms.

Note in this case we have a natural identification

$$
\mathcal{M}^{\vee, \mathcal{e}} \underset{\mathcal{e}}{\otimes} \mathcal{N} \simeq \text { Funct }_{\mathcal{E}-\bmod }(\mathcal{M}, \mathcal{N}), \quad \mathcal{N} \in \mathcal{C}-\bmod
$$

D.1.3. Example. Take $\mathcal{M}=\mathcal{C} \otimes \mathcal{M}_{0}$ where $\mathcal{M}_{0}$ is a plain DG category. If $\mathcal{M}_{0}$ is dualizable as a DG category, then $\mathcal{M}$ is dualizable as a $\mathcal{C}$-module and

$$
\mathcal{M}^{\vee, e} \simeq \mathcal{C} \otimes \mathcal{M}_{0}^{\vee}
$$

D.1.4. Let $m \in \mathcal{M}$ be an object. We shall say that $m$ is ULA over $\mathcal{C}$ if there exists an object $m^{\vee, \mathrm{e}} \in \mathcal{M}^{\vee, \mathrm{e}}$ equipped with a functorial identification

$$
\operatorname{Maps}_{\mathcal{M}}\left(c \otimes m, m^{\prime}\right) \simeq \operatorname{Maps}_{\mathcal{C}}\left(c, \operatorname{counit}_{\mathcal{M}}^{\mathcal{e}}\left(m^{\prime} \underset{\mathcal{C}}{\otimes} m^{\vee, \mathcal{e}}\right)\right), \quad c \in \mathcal{C}, m^{\prime} \in \mathcal{M} .
$$

The datum of such an identification is equivalent to that of a pair of maps

$$
\mathbf{1}_{\mathcal{C}} \stackrel{\mu}{\rightarrow} \operatorname{counit}_{\mathcal{M}}^{\mathfrak{e}}\left(m \underset{\mathcal{C}}{\otimes} m^{\vee, \mathcal{e}}\right) \text { and } m^{\vee, \mathcal{A}} \underset{\mathcal{C}}{\otimes} m \stackrel{\epsilon}{\rightarrow} \operatorname{unit}_{\mathcal{M}}^{\mathfrak{e}}\left(\mathbf{1}_{\mathcal{C}}\right)
$$

such that the composite

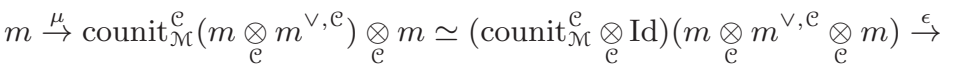

$$
\begin{aligned}
& \rightarrow\left(\operatorname{counit}_{\mathcal{M}}^{\mathfrak{e}} \underset{\mathcal{C}}{\otimes} \operatorname{Id}\right)\left(m \underset{\mathcal{e}}{\otimes} \operatorname{unit}_{\mathcal{M}}^{\mathfrak{e}}\left(\mathbf{1}_{\mathcal{C}}\right)\right) \simeq m
\end{aligned}
$$

is the identity map, and similarly for $m^{\vee, e}$.

Note that if an object $m \in \mathcal{M}$ is ULA, then so is the corresponding $m^{\vee, e} \in \mathcal{M}^{\vee, e}$ with

$$
\left(m^{\vee, \mathcal{e}}\right)^{\vee, \mathcal{e}} \simeq m \text {. }
$$


D.1.5. In what follows we will assume that $\mathcal{C}$ and $\mathcal{M}$ are compactly generated (as DG categories). We will also assume that the unit object $\mathbf{1}_{\mathcal{C}}$ is compact. (But we do not assume that the tensor product operation on $\mathcal{C}$ or the action of $\mathcal{C}$ on $\mathcal{M}$ preserve compactness.)

Note that for a pair of objects $m, m^{\prime} \in M$, we have a well-defined $\underline{\operatorname{Hom}}\left(m, m^{\prime}\right) \in \mathcal{C}$, satisfying

$$
\operatorname{Maps}_{\mathfrak{C}}\left(c, \underline{\operatorname{Hom}}\left(m, m^{\prime}\right)\right):=\operatorname{Maps}_{\mathcal{M}}\left(c \otimes m, m^{\prime}\right) \text {. }
$$

For a fixed $m$, the functor $m^{\prime} \mapsto \underline{\operatorname{Hom}}\left(m, m^{\prime}\right)$ preserves colimits if and only if $c \otimes m$ are compact for all compact $c \in \mathcal{C}$; in particular in this case $m$ itself is compact.

For $c \in \mathcal{C}$, we have a tautological map

$$
c \otimes \underline{\operatorname{Hom}}\left(m, m^{\prime}\right) \rightarrow \underline{\operatorname{Hom}}\left(m, c \otimes m^{\prime}\right) .
$$

We have:

Lemma D.1.6. An object $m \in \mathcal{M}$ is $U L A$ if and only if the functor

$$
m^{\prime} \mapsto \underline{\operatorname{Hom}}\left(m, m^{\prime}\right), \quad \mathcal{M} \rightarrow \mathcal{C}
$$

preserves colimits and (D.3) is an isomorphism for all $c \in \mathcal{C}$.

Proof. If $m$ is ULA we have

$$
\underline{\operatorname{Hom}}\left(m, m^{\prime}\right) \simeq \operatorname{counit}_{\mathcal{M}}^{\mathrm{e}}\left(m^{\prime} \underset{\mathrm{e}}{\otimes} m^{\vee, \mathcal{e}}\right) .
$$

The other direction follows from (D.1) for $\mathcal{N}=\mathcal{C}$.

Corollary D.1.7. If $\mathcal{C}$ is rigid, any compact $m \in \mathcal{M}$ is $U L A$.

Proof. It is enough to check that the objects $c \otimes m$ are compact and the maps (D.3) are isomorphisms for $c$ that are dualizable in $\mathcal{C}$. In the rigid case this is automatic.

D.2. Adding self-duality. Let $\mathcal{C}$ be as above (a compactly generated symmetric monoidal DG category with a compact unit).

D.2.1. Assume that $\mathcal{C}$ contains a compact object, to be denoted $\widetilde{\mathbf{1}}_{\mathcal{C}}$, so that the pairing

$$
\mathcal{C} \otimes \mathcal{C} \mapsto \text { Vect }, \quad c_{1}, c_{2} \mapsto \mathcal{H} \text { ome }\left(\widetilde{\mathbf{1}}_{\mathcal{C}}, c_{1} \otimes c_{2}\right)
$$

defines a self-duality

$$
e^{\vee} \simeq \mathcal{C}
$$

Let $\mathbb{D}_{\mathcal{C}}$ denote the corresponding contravariant self-equivalence on $\mathcal{C}^{c}$, i.e.,

$$
\left.\mathcal{H}_{\mathrm{e}}\left(\mathbb{D}_{\mathcal{e}}\left(c_{1}\right), c_{2}\right)\right) \simeq \mathcal{H}_{\mathrm{C}}\left(\widetilde{\mathbf{1}}_{\mathfrak{e}}, c_{1} \otimes c_{2}\right), \quad c_{1} \in \mathcal{C}^{c}
$$

By construction

$$
\widetilde{\mathbf{1}}_{\mathfrak{e}} \simeq \mathbb{D}_{\mathfrak{e}}\left(\mathbf{1}_{\mathfrak{e}}\right)
$$

D.2.2. Example. Let $\mathcal{C}=\mathrm{D}-\bmod (Y)$, where $Y$ is a scheme of finite type over a ground field $k$ of characteristic 0 . We regard $\mathcal{C}$ as a symmetric monoidal category via the $\stackrel{!}{\otimes}$ tensor product.

Then we can take $\widetilde{\mathbf{1}}_{\mathcal{e}}$ to be the "constant sheaf" $k_{Y} \in \mathrm{D}-\bmod (Y)$, i.e., the Verdier dual of the dualizing sheaf $\omega y$. The functor $\mathbb{D}_{e}$ is the Verdier duality self-equivalence of $\mathrm{D}-\bmod (Y)^{c}$, see Sect. D.3.1.

D.2.3. Let $\mathcal{M}$ be a $\mathcal{C}$-module category. Note that for a pair of objects $m, m^{\prime} \in \mathcal{M}$ and $c \in \mathcal{C}^{c}$ we have a canonically defined map

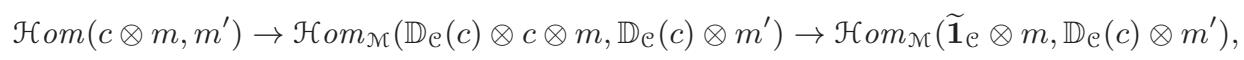

where the last arrow comes from the canonical map

$$
\widetilde{\mathbf{1}}_{\mathcal{C}} \rightarrow \mathbb{D}_{\mathcal{C}}(c) \otimes c .
$$


D.2.4. Assume that $\mathcal{M}$ is compactly generated as a DG category. Let $\mathbb{D}_{\mathcal{M}}$ denote the canonical contravariant equivalence

$$
\mathcal{M}^{c} \rightarrow\left(\mathcal{M}^{\vee}\right)^{c}
$$

Let $\langle-,-\rangle$ denote the tautological pairing

$$
\mathcal{M} \times \mathcal{M}^{\vee} \rightarrow \text { Vect }
$$

Consider $\mathcal{M}^{\vee}$ as a $\mathcal{C}$-module category, where the action of $c \in \mathcal{C}$ on $\mathcal{M}^{\vee}$ is the functor dual to that of the action of $c$ on $\mathcal{M}$.

Define a functor

$$
\begin{aligned}
&\langle-,-\rangle_{\mathcal{C}}: \mathcal{M} \times \mathcal{M}^{\vee} \rightarrow \mathcal{C} \\
& \mathcal{H}_{o} m_{\mathcal{C}}\left(c,\left\langle m, m^{\prime}\right\rangle_{\mathcal{C}}\right):=\left\langle\mathbb{D}_{\mathcal{C}}(c) \otimes m, m^{\prime}\right\rangle \simeq\left\langle m, \mathbb{D}_{\mathcal{C}}(c) \otimes m^{\prime}\right\rangle, \quad m \in \mathcal{M}, m^{\prime} \in \mathcal{M}^{\vee} .
\end{aligned}
$$

By construction, (D.6) factors via a functor

$$
\mathcal{M} \underset{\mathrm{C}}{\otimes} \mathcal{M}^{\vee} \rightarrow \mathcal{C}
$$

D.2.5. Assume that (D.7) is the counit of a duality, so we find ourselves in the context of Sect. D.1 In particular, we can identify

$$
\mathcal{M}^{\vee, e} \simeq \mathcal{M}^{\vee}
$$

D.2.6. We claim:

Proposition D.2.7. For $m \in \mathcal{M}^{c}$ the following conditions are equivalent:

(i) The object $m$ is ULA over $\mathcal{C}$;

(ii) The object $m$ is ULA, with

$$
m^{\vee, \mathcal{e}}=\mathbb{D}_{\mathcal{M}}\left(\widetilde{\mathbf{1}}_{\mathcal{C}} \otimes m\right)
$$

and $\mu$ being the canonical map

$$
\mathbf{1}_{\mathcal{C}} \rightarrow\left\langle m, \mathbb{D}_{\mathcal{M}}\left(\widetilde{\mathbf{1}}_{\mathcal{C}} \otimes m\right)\right\rangle_{\mathcal{e}}
$$

(iii) The object $\widetilde{\mathbf{1}}_{\mathcal{C}} \otimes m$ is compact, and the map (D.5) is an isomorphism for every $c \in \mathcal{C}^{c}$ and $m^{\prime} \in \mathcal{M}$.

Proof. The implication (ii) $\Rightarrow$ (i) is tautological. To prove (iii) $\Rightarrow$ (ii) we note that if (D.5) is an isomorphism, then the object $\mathbb{D}_{\mathcal{M}}\left(\widetilde{\mathbf{1}}_{\mathcal{C}} \otimes m\right)$ satisfies the requirements of $m^{\vee, e}$. It remains prove (i) $\Rightarrow$ (iii).

Let $m$ be ULA over $\mathcal{C}$, and let $m^{\vee, \mathcal{e}}$ be the corresponding object of $\mathcal{M}^{\vee, \mathcal{e}}$, which we now identify with $\mathcal{M}^{\vee}$. We have:

$$
\mathcal{H}_{\operatorname{com}}\left(c \otimes m, m^{\prime}\right) \simeq \mathcal{M}_{\mathcal{M}}\left(c s_{\mathfrak{C}}\left(c,\left\langle m^{\prime}, m^{\vee, \mathcal{e}}\right\rangle_{\mathfrak{e}}\right) \simeq\left\langle\mathbb{D}_{\mathcal{C}}(c) \otimes m^{\prime}, m^{\vee, \mathcal{e}}\right\rangle, \quad c \in \mathfrak{C}^{c}, m^{\prime} \in \mathcal{M} .\right.
$$

In particular, taking $c=\widetilde{\mathbf{1}}_{\mathcal{e}}$, we obtain

$$
\mathcal{H}_{o} m_{\mathcal{M}}\left(\widetilde{\mathbf{1}}_{\mathcal{C}} \otimes m, m^{\prime}\right) \simeq\left\langle m^{\prime}, m^{\vee, \mathcal{e}}\right\rangle, \quad m^{\prime} \in \mathcal{M} .
$$

Hence, we obtain that $m^{\vee, \mathcal{C}}$ is compact and

$$
\mathbb{D}_{\mathcal{M}}\left(m^{\vee, \mathcal{e}}\right) \simeq \widetilde{\mathbf{1}}_{\mathcal{C}} \otimes m
$$

in particular $\widetilde{\mathbf{1}}_{\mathcal{C}} \otimes m$ is compact.

With respect to the last identification, the map (D.5) becomes the map

$$
\begin{aligned}
\mathcal{H}_{o m_{\mathcal{M}}}\left(c \otimes m, m^{\prime}\right) \simeq \mathcal{M}_{\operatorname{Maps}_{\mathcal{C}}(c,} & \left.\left\langle m^{\prime}, m^{\vee, \mathcal{e}}\right\rangle_{\mathcal{C}}\right) \simeq\left\langle\mathbb{D}_{\mathcal{C}}(c) \otimes m^{\prime}, m^{\vee, \mathcal{e}}\right\rangle \simeq \\
& \simeq \mathcal{H} \operatorname{Hom}_{\mathcal{M}}\left(\mathbb{D}_{\mathcal{M}}\left(m^{\vee, \mathcal{e}}\right), \mathbb{D}_{\mathcal{C}}(c) \otimes m^{\prime}\right) \simeq \mathcal{H} \text { om } m_{\mathcal{M}}\left(\widetilde{\mathbf{1}}_{\mathcal{C}} \otimes m, \mathbb{D}_{\mathcal{C}}(c) \otimes m^{\prime}\right),
\end{aligned}
$$

and hence is an isomorphism. 
D.2.8. Example. Let $\mathcal{M}$ be of the form $\mathcal{C} \otimes \mathcal{M}_{0}$ for a compactly generated DG category $\mathcal{M}_{0}$. Then the pairing (D.7) does indeed define the counit of a duality. The composite identification

$$
\mathcal{C}^{\vee} \otimes \mathcal{M}_{0}^{\vee} \simeq\left(\mathcal{C} \otimes \mathcal{M}_{0}\right)^{\vee} \simeq\left(\mathcal{C} \otimes \mathcal{M}_{0}\right)^{\vee, \mathcal{e}} \simeq \mathcal{C} \otimes \mathcal{M}_{0}^{\vee}
$$

is given by the self-duality (D.4).

D.3. The ULA condition in the geometric situation. In this subsection we will take $\mathcal{C}$ to be $\mathrm{D}-\bmod (Y)$, where $Y$ is a scheme of finite type. Let $f: Z \rightarrow Y$ be a scheme over $Y$; take $\mathcal{M}:=\operatorname{D}-\bmod (Z)$, which is acted on by $\mathrm{D}-\bmod (Y)$ via

$$
\mathcal{F}_{Y}, \mathcal{F}_{Z} \mapsto f^{!}\left(\mathcal{F}_{Y}\right) \stackrel{!}{\otimes} \mathcal{F}_{Z}
$$

We will bring the concept of ULA developed above in contact with the more familiar geometric geometric notion.

D.3.1. First, we note that $D-\bmod (Y)$ fits into the paradigm of Sect. D.2.1 with

$$
\mathbb{D}_{\mathrm{D}-\bmod (Y)}:=\mathbb{D}_{Y}^{\text {Verdier }} \text {. }
$$

The corresponding object $\widetilde{\mathbf{1}}_{\mathrm{D}-\bmod (Y)}$ is $\mathrm{e}_{Y}$, the "constant sheaf", i.e.,

$$
\mathcal{H} \text { om }\left(\mathrm{e}_{Y}, \mathcal{F}\right)=\Gamma_{\mathrm{dR}}(Y, \mathcal{F}) .
$$

Similarly, we use Verdier duality on $Z$ to identify $\mathrm{D}-\bmod (Z)^{\vee}$ with $\mathrm{D}-\bmod (Z)$.

In this case, the pairing (D.6) identifies with

$$
\left\langle\mathcal{F}_{1}, \mathcal{F}_{2}\right\rangle_{\operatorname{D}-\bmod (Y)}=f_{*}\left(\mathcal{F}_{1} \stackrel{!}{\otimes} \mathcal{F}_{2}\right) .
$$

D.3.2. We claim that the resulting pairing (D.7) provides the counit of the adjunction.

Indeed, the unit map

$$
\mathrm{D}-\bmod (Y) \rightarrow \mathrm{D}-\bmod (Z) \underset{\mathrm{D}-\bmod (Y)}{\otimes} \mathrm{D}-\bmod (Z)
$$

is determined by the condition that the corresponding object

$$
\operatorname{unit}_{\mathrm{D}-\bmod (Z)}^{\mathrm{D}-\bmod (Y)}\left(\mathbf{1}_{\mathrm{D}-\bmod (Y)}\right) \in \mathrm{D}-\bmod (Z) \underset{\mathrm{D}-\bmod (Y)}{\otimes} \mathrm{D}-\bmod (Z) \simeq \mathrm{D}-\bmod (Z \underset{Y}{\times} Z)
$$

equals

where

$$
\left(\Delta_{Z / Y}\right)_{*}\left(\omega_{Z}\right)
$$

$$
\Delta_{Z / Y}: Z \rightarrow \underset{Y}{Z} \underset{Y}{\times}
$$

is the relative diagonal map.

D.3.3. Thus, from Proposition D.2.7 we obtain:

Corollary D.3.4. For a compact object $\mathcal{F} \in \mathrm{D}-\bmod (Z)$ the following conditions are equivalent:

(i) There exists an object $\mathcal{F}^{\vee} \in \mathrm{D}-\bmod (Z)^{c}$ equipped with maps

$$
\mu: \omega_{Y} \rightarrow f_{*}\left(\mathcal{F} \stackrel{!}{\otimes} \mathcal{F}^{\vee}\right) \text { and } \epsilon: \mathcal{F}^{\vee} \underset{Y}{\otimes} \mathcal{F} \rightarrow\left(\Delta_{Z / Y}\right)_{*}\left(\omega_{Z}\right)
$$

(here $-\underset{Y}{\otimes}-$ stands for the !-pullback of $-\underset{\square}{-}$ along $\underset{Y}{Z} \underset{Y}{\times} \rightarrow Z \times Z$ ) such that the composite

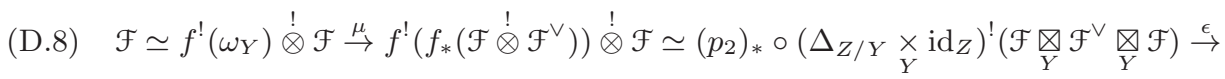

$$
\rightarrow\left(p_{2}\right)_{*} \circ\left(\Delta_{Z / Y} \underset{Y}{\times} \operatorname{id}_{Z}\right)^{!}\left(\mathcal{F} \underset{Y}{\underset{X}{\otimes}}\left(\Delta_{Z / Y}\right)_{*}\left(\omega_{Z}\right)\right) \simeq \mathcal{F}
$$

(here $p_{2}$ denotes the second projection $\underset{Y}{Z} \underset{Y}{\times} Z \rightarrow Z$ ) is the identity map, and similarly for $\mathcal{F}^{\vee}$. 
(ii) Same as (i), but for $\mathcal{F}^{\vee}=\mathbb{D}_{Z}^{\text {Verdier }}\left(f^{!}\left(\mathrm{e}_{Y}\right) \stackrel{!}{\otimes} \mathcal{F}\right)$ (in particular, $f^{!}\left(\mathrm{e}_{Y}\right) \stackrel{!}{\otimes} \mathcal{F}$ is compact), with $\mu$ being the map

$$
\omega_{Y} \rightarrow f_{*}\left(\mathcal{F} \stackrel{!}{\otimes} \mathbb{D}_{Z}^{\text {Verdier }}\left(f^{!}\left(\mathrm{e}_{Y}\right) \stackrel{!}{\otimes} \mathcal{F}\right)\right)
$$

corresponding to the identity element under

$$
\begin{aligned}
& \mathcal{H} \operatorname{Hom}_{\mathrm{D}-\bmod (Y)}\left(\omega_{Y}, f_{*}\left(\mathcal{F} \dot{\bullet} \mathbb{D}_{Z}^{\text {Verdier }}\left(f^{!}\left(\mathrm{e}_{Y}\right) \stackrel{!}{\otimes} \mathcal{F}\right)\right)\right) \simeq \Gamma_{\mathrm{dR}}\left(Y, \mathrm{e}_{Y} \stackrel{!}{\otimes} f_{*}\left(\mathcal{F} \stackrel{!}{\otimes} \mathbb{D}_{Z}^{\text {Verdier }}\left(f^{!}\left(\mathrm{e}_{Y}\right) \stackrel{!}{\otimes} \mathcal{F}\right)\right)\right) \simeq
\end{aligned}
$$

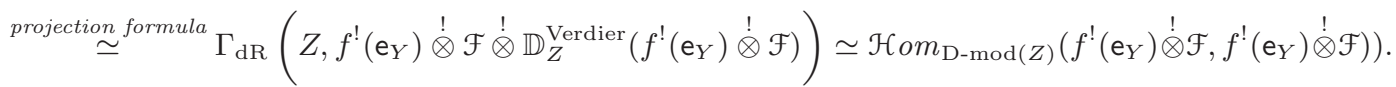

(iii) For any $\mathcal{F}_{Y} \in \mathrm{D}-\bmod (Y)^{c}$ and $\mathcal{F}^{\prime} \in \mathrm{D}-\bmod (Z)$, the map

$$
\begin{aligned}
& \mathcal{H} \operatorname{mom}_{\mathrm{D}-\bmod (Z)}\left(f^{!}\left(\mathcal{F}_{Y}\right) \stackrel{!}{\otimes} \mathcal{F}, \mathcal{F}^{\prime}\right) \rightarrow \\
& \rightarrow \mathcal{H} \operatorname{Hom}_{\mathrm{D}-\bmod (Z)}\left(f^{!}\left(\mathbb{D}_{Y}^{\text {Verdier }}\left(\mathcal{F}_{Y}\right)\right) \stackrel{!}{\otimes} f^{!}\left(\mathcal{F}_{Y}\right) \stackrel{!}{\otimes} \mathcal{F}, f^{!}\left(\mathbb{D}_{Y}^{\text {Verdier }}\left(\mathcal{F}_{Y}\right)\right) \stackrel{!}{\otimes} \mathcal{F}^{\prime}\right) \rightarrow \\
& \rightarrow \mathcal{H} \operatorname{mom}_{\mathrm{D}-\bmod (Z)}\left(f^{!}\left(\mathrm{e}_{Y}\right) \stackrel{!}{\otimes} \mathcal{F}, f^{!}\left(\mathbb{D}_{Y}^{\text {Verdier }}\left(\mathcal{F}_{Y}\right)\right) \stackrel{!}{\otimes} \mathcal{F}^{\prime}\right)
\end{aligned}
$$

is an isomorphism.

D.3.5. We will call objects of $\mathrm{D}-\bmod (Z)$ satisfying the equivalent conditions of Corollary D.3.4 ULA over $Y$ (or ULA with respect to $f$ ).

Remark D.3.6. Note that condition (iii) in Corollary D.3.4 can be rephrased as follows:

For $\mathcal{F}_{Y} \in \mathrm{D}-\bmod (Y)$, the object

$$
f^{*}\left(\mathcal{F}_{Y}\right) \stackrel{*}{\otimes}\left(f^{!}\left(\mathrm{e}_{Y}\right) \stackrel{!}{\otimes} \mathcal{F}\right) \in \mathrm{D}-\bmod (Z)
$$

is defined and the canonical map

$$
f^{*}\left(\mathcal{F}_{Y}\right) \stackrel{*}{\otimes}\left(f^{!}\left(\mathrm{e}_{Y}\right) \stackrel{!}{\otimes} \mathcal{F}\right) \rightarrow f^{!}\left(\mathcal{F}_{Y}\right) \stackrel{!}{\otimes} \mathcal{F}
$$

is an isomorphism.

D.3.7. When we view this definition from the point of view of condition (i), we obtain the following assertion that reads that "local acyclicity implies universal local acyclicity":

Corollary D.3.8. Let $g: Y^{\prime} \rightarrow Y$ be a morphism of schemes, and let set $Z^{\prime}:=Y_{Y}^{\prime} \underset{Y}{\times}$. If $\mathcal{F}$ is $U L A$ over $Y$, then its!-pullback to $Z^{\prime}$ is ULA over $Y^{\prime}$.

In the context of $\ell$-adic sheaves, an assertion parallel to Corollary D.3.8 had been proved by O. Gabber, see [LZ, Corollary 6.6].

D.4. An aside: the notion of ULA in other sheaf-theoretic contexts. We will now place ourselves in a general sheaf-theoretic context of Sect. A.1.1 excluding (a) (the exclusion is because we want our categories to be compactly generated).

D.4.1. Let $f: Z \rightarrow Y$ be a map of schemes. We consider $\operatorname{Shv}(Z)$ as a module category over $\operatorname{Shv}(Y)$ via the operation

$$
f^{!}(-) \stackrel{!}{\otimes}-
$$

However, the notion of ULA developed from Sect. D.1 is not directly applicable in this context, since $\operatorname{Shv}(Z)$ is not in general dualizable as a $\operatorname{Shv}(Y)$-module category. However, we claim that the following analog of Corollary D.3.4 holds: 
Proposition D.4.2. For a compact object $\mathcal{F} \in \operatorname{Shv}(Z)$ the following conditions are equivalent:

(i) There exists an object $\mathcal{F}^{\vee} \in \operatorname{Shv}(Z)^{c}$ equipped with maps

$$
\mu: \omega_{Y} \rightarrow f_{*}\left(\mathcal{F} \stackrel{!}{\otimes} \mathcal{F}^{\vee}\right) \text { and } \epsilon: \mathcal{F}^{\vee} \underset{Y}{\otimes} \mathcal{F} \rightarrow\left(\Delta_{Z / Y}\right)_{*}\left(\omega_{Z}\right)
$$

such that the composite

$$
\begin{aligned}
& \mathcal{F} \simeq f^{!}\left(\omega_{Y}\right) \stackrel{!}{\otimes} \mathcal{F} \stackrel{\mu}{\rightarrow} f^{!}\left(f_{*}\left(\mathcal{F} \stackrel{!}{\otimes} \mathcal{F}^{\vee}\right)\right) \stackrel{!}{\otimes} \mathcal{F} \simeq\left(p_{2}\right)_{*} \circ\left(\Delta_{Z / Y} \underset{Y}{\times} \operatorname{id}_{Z}\right)^{!}(\mathcal{F} \underset{Y}{\underset{\mathcal{F}}{\vee}} \underset{Y}{\underset{\mathcal{F}}{\otimes}} \mathcal{F} \stackrel{\epsilon}{\rightarrow} \\
& \rightarrow\left(p_{2}\right)_{*} \circ\left(\Delta_{Z / Y} \underset{Y}{\times} \operatorname{id}_{Z}\right)^{!}\left(\mathcal{F} \underset{Y}{\underset{Y}{\otimes}}\left(\Delta_{Z / Y}\right)_{*}\left(\omega_{Z}\right)\right) \simeq \mathcal{F}
\end{aligned}
$$

is the identity map, and similarly for $\mathcal{F}^{\vee}$.

(ii) Same as (i), but for $\mathcal{F}^{\vee}=\mathbb{D}_{Z}^{\text {Verdier }}\left(f^{!}\left(\mathrm{e}_{Y}\right) \stackrel{!}{\otimes} \mathcal{F}\right)$ with $\mu$ being the canonical map

$$
\omega_{Y} \rightarrow f_{*}\left(\mathcal{F} \stackrel{!}{\otimes} \mathbb{D}_{Z}^{\text {Verdier }}\left(f^{!}\left(\mathrm{e}_{Y}\right) \stackrel{!}{\otimes} \mathcal{F}\right)\right)
$$

(iii) For any base change

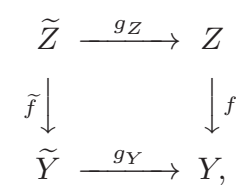

and $\widetilde{\mathcal{F}}:=g_{Z}^{!}(\mathcal{F})$, for any $\mathcal{F}_{\widetilde{Y}} \in \operatorname{Shv}(\widetilde{Y})^{c}$ and $\widetilde{\mathcal{F}}^{\prime} \in \operatorname{Shv}(\widetilde{Z})$, the map

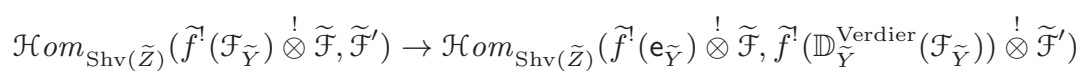

is an isomorphism.

Proof. Clearly, (ii) implies (i). Let us show that (iii) implies (ii).

First, note that the RHS in (D.9) a priori identifies with

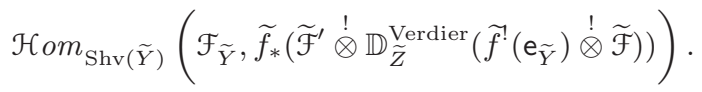

Take $\tilde{Y}:=Z$; so that the square D.11 becomes

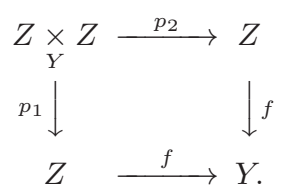

Take $\widetilde{\mathcal{F}}^{\prime}:=\left(\Delta_{Z / Y}\right)_{*}\left(\omega_{Z}\right)$ and and $\mathcal{F}_{\widetilde{Y}}:=\mathbb{D}_{Z}^{\text {Verdier }}\left(f^{!}\left(\mathrm{e}_{Y}\right) \stackrel{!}{\otimes} \mathcal{F}\right)$. The expression in (D.13) identifies with

$$
\left.\mathcal{H}_{\operatorname{Hom}} m_{\mathrm{Shv}(Z)}\left(\mathbb{D}_{Z}^{\text {Verdier }}\left(f^{!}\left(\mathrm{e}_{Y}\right) \stackrel{!}{\otimes} \mathcal{F}\right), \mathbb{D}_{Z}^{\text {Verdier }}\left(f^{!}\left(\mathrm{e}_{Y}\right) \stackrel{!}{\otimes} \mathcal{F}\right)\right)\right) .
$$

The required map $\epsilon$ is obtained via (D.9) from the identity element in (D.14).

Finally, let us assume (i) and deduce (iii). If we have a datum as in (i) for $f: Z \rightarrow Y$, its !-pullback defines a similar data for $\widetilde{f}: \widetilde{Z} \rightarrow \widetilde{Y}$. So we can assume that $g=\mathrm{id}$. 
The datum of $\mu$ defines a map

$$
\begin{aligned}
& \mathcal{H}^{\circ o m_{\operatorname{Shv}(Z)}}\left(f^{!}\left(\mathcal{F}_{Y}\right) \stackrel{!}{\otimes} \mathcal{F}, \mathcal{F}^{\prime}\right) \rightarrow \mathcal{H} \operatorname{om}_{\operatorname{Shv}(Z)}\left(f^{!}\left(\mathcal{F}_{Y}\right) \stackrel{!}{\otimes} \mathcal{F} \stackrel{!}{\otimes} \mathcal{F}^{\vee}, \mathcal{F}^{\prime} \stackrel{!}{\otimes} \mathcal{F}^{\vee}\right) \rightarrow \\
& \rightarrow \mathcal{H}^{\circ o m_{\operatorname{Shv}}(Y)}\left(f_{*}\left(f^{!}\left(\mathcal{F}_{Y}\right) \stackrel{!}{\otimes} \mathcal{F} \dot{\otimes} \mathcal{F}^{\vee}\right), f_{*}\left(\mathcal{F}^{\prime} \dot{\otimes} \mathcal{F}^{\vee}\right)\right) \simeq
\end{aligned}
$$

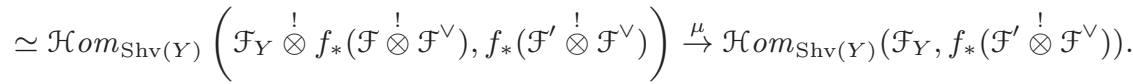

The datum of $\epsilon$ defines an inverse map

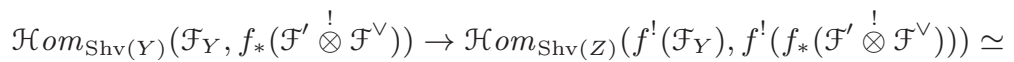

$$
\begin{aligned}
& \simeq \mathcal{H}_{\operatorname{com}}^{\operatorname{Shv}(Z)}\left(f^{!}\left(\mathcal{F}_{Y}\right), p_{2 *}\left(p_{1}^{!}\left(\mathcal{F}^{\prime} \stackrel{!}{\otimes} \mathcal{F}^{\vee}\right)\right)\right) \rightarrow \mathcal{H} \operatorname{Hom}_{\operatorname{Shv}(Z)}\left(f^{!}\left(\mathcal{F}_{Y}\right) \stackrel{!}{\otimes} \mathcal{F}, p_{2 *}\left(p_{1}^{!}\left(\mathcal{F}^{\prime} \stackrel{!}{\otimes} \mathcal{F}^{\vee}\right)\right) \stackrel{!}{\otimes} \mathcal{F}\right) \simeq \\
& \simeq \mathcal{H}^{\circ} m_{\operatorname{Shv}(Z)}\left(f^{!}\left(\mathcal{F}_{Y}\right) \stackrel{!}{\otimes} \mathcal{F}, p_{2 *}\left(p_{1}^{!}\left(\mathcal{F}^{\prime} \stackrel{!}{\otimes} \mathcal{F}^{\vee}\right) \stackrel{!}{\otimes} p_{2}^{!}(\mathcal{F})\right)\right) \simeq \\
& \left.\simeq \mathcal{H}^{\circ o m_{\operatorname{Shv}}(Z)}\left(f^{!}\left(\mathcal{F}_{Y}\right) \stackrel{!}{\otimes} \mathcal{F}, p_{2 *}\left(p_{1}^{!}\left(\mathcal{F}^{\vee}\right) \stackrel{!}{\otimes} p_{2}^{!}(\mathcal{F})\right) \stackrel{!}{\otimes} p_{1}^{!}\left(\mathcal{F}^{\prime}\right)\right)\right) \stackrel{\epsilon}{\rightarrow} \\
& \rightarrow \mathcal{H}^{\circ o m_{\operatorname{Shv}}(Z)}\left(f^{!}\left(\mathcal{F}_{Y}\right) \stackrel{!}{\otimes} \mathcal{F}, p_{2 *}\left(\left(\Delta_{Z / Y}\right)_{*}\left(\omega_{Z}\right) \stackrel{!}{\otimes} p_{1}^{!}\left(\mathcal{F}^{\prime}\right)\right)\right) \simeq \mathcal{H} \operatorname{Hom}_{\operatorname{Shv}(Z)}\left(f^{!}\left(\mathcal{F}_{Y}\right) \stackrel{!}{\otimes} \mathcal{F}, \mathcal{F}^{\prime}\right) .
\end{aligned}
$$

In particular, taking $\mathcal{F}_{Y}=\mathrm{e}_{Y}$, we obtain an identification between

$$
\mathcal{H}_{\operatorname{Hom}} m_{\operatorname{Shv}(Z)}\left(\mathbb{D}_{Z}^{\text {Verdier }}(\mathcal{F}), \mathcal{F}^{\prime}\right) \simeq \Gamma_{\mathrm{dR}}\left(Z, \mathcal{F}^{\prime} \stackrel{!}{\otimes} \mathcal{F}^{\vee}\right) \simeq \Gamma_{\mathrm{dR}}\left(Y, f_{*}\left(\mathcal{F}^{\prime} \stackrel{!}{\otimes} \mathcal{F}^{\vee}\right)\right) \simeq \mathcal{H} \operatorname{Com}_{\mathrm{Shv}(Y)}\left(\mathrm{e}_{Y}, f_{*}\left(\mathcal{F}^{\prime} \stackrel{!}{\otimes}^{\mathcal{F}^{\vee}}\right)\right)
$$

and

$$
\mathcal{H}^{\circ o m_{\operatorname{Shv}}(Z)}\left(f^{!}\left(\mathrm{e}_{Y}\right) \stackrel{!}{\otimes} \mathcal{F}, \mathcal{F}^{\prime}\right),
$$

functorial in $\mathcal{F}^{\prime}$. Hence,

$$
\mathcal{F}^{\vee} \simeq \mathbb{D}_{Z}^{\text {Verdier }}\left(f^{!}\left(\mathrm{e}_{Y}\right) \stackrel{!}{\otimes} \mathcal{F}\right)
$$

Under this identification, the map (D.12) goes over to the composition of (D.15) and

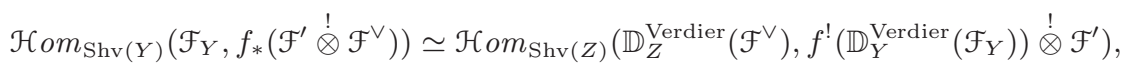

hence it is an isomorphism.

D.4.3. We will call objects of $\mathrm{D}-\bmod (Z)$ satisfying the equivalent conditions of Proposition D.4.2 ULA over $Y$ (or ULA with respect to $f$ ).

Remark D.4.4. Let us compare the notion of ULA in the three contexts (b), (b') and (b"). Note that we have fully faithful embeddings of the corresponding categories $\left(\mathrm{b}^{\prime \prime}\right) \rightarrow\left(\mathrm{b}^{\prime}\right) \rightarrow(\mathrm{b})$.

We note that if $\mathcal{F}$ is an object of a smaller category, then, if we view the from the point of view of condition (ii) of Proposition D.3.4 it is ULA in the smaller category if and only if it is such from the point of view of a bigger category.

Note that this property is non-obvious from the point of view of condition (iii) of Proposition D.3.4 since for the larger category we are testing the isomorphism on a larger collection of objects (one that are denoted $\widetilde{\mathcal{F}}^{\prime}$ ).

Remark D.4.5. Note that the property of being ULA is by design stable under base change: this is obvious from the point of view of each of the conditions (i), (ii), (iii) in Proposition D.3.4

That said, the proof of Proposition D.3.4 shows that it is enough to require that the map (D.12) be an isomorphism for $\left(\tilde{Y}, g_{Y}\right)$ being the pair $(Z, f)$. 
D.5. The ULA property for D-modules. In this subsection we will come back to the ULA property in the context of D-modules.

We will be working over a ground field $k$ of characteristic 0 . Let $Y$ be a smooth scheme of finite type over $k$. Consider the symmetric monoidal category $\mathcal{C}:=\mathrm{D}-\bmod (Y)$. In this subsection we will give a hands-on criterion for objects (in some class of $\mathrm{D}-\bmod (Y)$-module categories) to be ULA.

D.5.1. We will regard the category $\mathrm{D}-\bmod (Y)$ as $\mathrm{QCoh}\left(Y_{\mathrm{dR}}\right)$, where $Y_{\mathrm{dR}}$ is the de Rham prestack of $Y$, i.e.,

$$
\operatorname{Maps}\left(S, Y_{\mathrm{dR}}\right)=\operatorname{Maps}\left(S_{\mathrm{red}}, Y\right) .
$$

A key observation observation is that $Y_{\mathrm{dR}}$ is 1-affine (see [Ga5, Definition 1.3.7] for what this means, and [Ga5, Theorem 2.6.3] for the statement of the result).

In other words, a datum of a $\mathrm{QCoh}\left(Y_{\mathrm{dR}}\right)$-module category $\mathcal{M}$ is equivalent to a datum of a sheaf of categories over $Y_{\mathrm{dR}}$, i.e., an assignment

$$
\begin{gathered}
\left(S \stackrel{y}{\rightarrow} Y_{\mathrm{dR}}\right) \rightsquigarrow \mathcal{M}_{S, y} \in \mathrm{QCoh}(S)-\mathbf{m o d}, \quad S \in \mathrm{Sch}^{\text {aff }}, \\
\left(S^{\prime} \stackrel{f}{\rightarrow} S\right) \rightsquigarrow \mathcal{M}_{S^{\prime}, y \circ f} \simeq \mathrm{QCoh}\left(S^{\prime}\right) \underset{\mathrm{QCoh}(S)}{\otimes} \mathcal{M}_{S, y},
\end{gathered}
$$

equipped with a data of homotopy-compatibility for compositions.

Explicitly, each $\mathcal{M}_{S, y}$ is recovered as

$$
\mathcal{M}_{S, y} \simeq \mathrm{QCoh}(S) \underset{\mathrm{QCoh}\left(Y_{\mathrm{dR}}\right)}{\otimes} \mathcal{M} .
$$

Vice versa, $\mathcal{M}$ can be identified with

$$
\lim _{S \stackrel{y}{\rightarrow} Y_{\mathrm{dR}}} \mathcal{M}_{S, y}
$$

equipped with an action of

$$
\lim _{S \rightarrow Y_{\mathrm{dR}}} \mathrm{Q} \operatorname{Coh}(S)=\mathrm{Q} \operatorname{Coh}\left(Y_{\mathrm{dR}}\right) .
$$

We will denote by $\mathcal{M}_{Y}$ the value of this sheaf categories on $Y$, where we regard $Y$ as equipped with the canonical projection to $Y_{\mathrm{dR}}$. Explicitly,

$$
\mathcal{M}_{Y} \simeq \mathrm{QCoh}(Y) \underset{\mathrm{Q} \operatorname{Coh}\left(Y_{\mathrm{dR}}\right)}{\otimes} \mathcal{M}
$$

D.5.2. Examples. If $\mathcal{M}=\mathcal{M}_{0} \otimes \mathrm{D}-\bmod (Y)$, then $\mathcal{M}_{Y}=\mathcal{M}_{0} \otimes \mathrm{QCoh}(Y)$.

Let now $\mathcal{M}=\mathrm{QCoh}\left(Z_{\mathrm{dR}}\right)$. Then

$$
\mathcal{M}_{Y}=\mathrm{QCoh}\left(Z_{\mathrm{dR}} \underset{Y_{\mathrm{dR}}}{\times} Y\right) .
$$

If $Z \rightarrow Y$ is a smooth map, one can identify $\mathrm{QCoh}\left(Z_{\mathrm{dR}} \underset{Y_{\mathrm{dR}}}{\times} Y\right)$ with the derived category of (quasicoherent sheaves of) modules over the ring $\mathrm{D}_{Z / Y}$ of vertical differential operators; this is a subring of $\mathrm{D}_{Z}$, generated by functions and vertical vector fields, i.e., vector fields along the fibers of the map $Z \rightarrow Y$.

D.5.3. The adjoint pair

$$
\text { ind : } \mathrm{QCoh}(Y) \rightleftarrows \mathrm{QCoh}\left(Y_{\mathrm{dR}}\right): \text { oblv }
$$

induces an adjoint pair

$$
\text { ind : } \mathcal{M}_{Y} \rightleftarrows \mathcal{M} \text { : oblv. }
$$

The functor oblv is conservative (indeed, since $Y$ is smooth, any map $S \rightarrow Y_{\mathrm{dR}}$ can be lifted to $Y$ ).

In what follows we will assume that $\mathcal{M}_{Y}$ is compactly generated as a DG category. The conservativity of oblv implies that in this case $\mathcal{M}$ is also compactly generated. 
D.5.4. Since $\mathcal{M}_{Y}$ is compactly generated, and hence dualizable, all the categories $\mathcal{M}_{S, y}$ are dualizable (as DG categories, or equivalently, as QCoh $(S)$-module categories). The assignment

$$
(S, y) \mapsto \mathcal{M}_{S, y}^{\vee}
$$

is also a sheaf of categories over $Y_{\mathrm{dR}}$.

Set

$$
\mathcal{M}^{\vee, Y_{\mathrm{dR}}}:=\lim _{S \stackrel{y}{\rightarrow}_{\mathrm{dR}}} \mathcal{M}_{S, y}^{\vee}
$$

The 1-affineness of $Y_{\mathrm{dR}}$ implies that $\mathcal{M}^{\vee, Y_{\mathrm{dR}}}$ is the dual of $\mathcal{M}$ as a $\mathrm{QCoh}\left(Y_{\mathrm{dR}}\right)$-module category. Denote by $\langle-,-\rangle_{Y_{\mathrm{dR}}}$ the resulting pairing

$$
\mathcal{M} \times \mathcal{M}^{\vee, Y_{\mathrm{dR}}} \rightarrow \mathrm{QCoh}\left(Y_{\mathrm{dR}}\right) .
$$

Define the pairing

by

$$
\langle-,-\rangle: \mathcal{M} \times \mathcal{M}^{\vee, Y_{\mathrm{dR}}} \rightarrow \text { Vect }
$$

$$
\langle-,-\rangle:=\Gamma_{\mathrm{dr}}(Y,-) \circ\langle-,-\rangle_{Y_{\mathrm{dR}}},
$$

where $\Gamma_{\mathrm{dr}}(Y,-)$ is the functor of de Rham cohomology

$$
\mathrm{QCoh}\left(Y_{\mathrm{dR}}\right) \simeq \mathrm{D}-\bmod (Y) \rightarrow \operatorname{Vect} .
$$

Lemma D.5.5. The functor $\langle-,-\rangle$ defines an equivalence $\mathcal{M}^{\vee, Y_{\mathrm{dR}}} \simeq \mathcal{M}^{\vee}$.

Proof. The adjunction

$$
\left(\Delta_{Y}\right)_{\mathrm{dR}, *}: \mathrm{D}-\bmod (Y) \rightleftarrows \mathrm{D}-\bmod (Y) \otimes \mathrm{D}-\bmod (Y): \Delta_{Y}^{!}
$$

(here we identify $\mathrm{D}-\bmod (Y) \otimes \mathrm{D}-\bmod (Y) \simeq \mathrm{D}-\bmod (Y \times Y)$ ) defines an adjunction

$$
\begin{aligned}
& \mathcal{M}^{\vee, Y_{\mathrm{dR}}} \underset{\mathrm{D}-\bmod (Y)}{\otimes} \mathcal{M} \simeq\left(\mathcal{M}^{\vee, Y_{\mathrm{dR}}} \otimes \mathcal{M}\right) \underset{\mathrm{D}-\bmod (Y) \otimes \mathrm{D}-\bmod (Y)}{\otimes} \mathrm{D}-\bmod (Y) \rightleftarrows \\
& \rightleftarrows\left(\mathcal{M}^{\vee, Y_{\mathrm{dR}}} \otimes \mathcal{M}\right) \underset{\mathrm{D}-\bmod (Y) \otimes \mathrm{D}-\bmod (Y)}{\otimes}(\mathrm{D}-\bmod (Y) \otimes \mathrm{D}-\bmod (Y)) \simeq \mathcal{M}^{\vee, Y_{\mathrm{dR}}} \otimes \mathcal{M}
\end{aligned}
$$

We define the unit object

to be the image of the unit

$$
\text { unit }_{\mathcal{M}} \in \mathcal{M}^{\vee, Y_{\mathrm{dR}}} \otimes \mathcal{M}
$$

under the above left adjoint

$$
\operatorname{unit}_{\mathcal{M}}^{Y_{\mathrm{dR}}} \in \mathcal{M}^{\vee, Y_{\mathrm{dR}}} \underset{\mathrm{D}-\bmod (Y)}{\otimes} \mathcal{M}
$$

$$
\mathcal{M}^{\vee, Y_{\mathrm{dR}}} \underset{\mathrm{D}-\bmod (Y)}{\otimes} \mathcal{M} \rightarrow \mathcal{M}^{\vee, Y_{\mathrm{dR}}} \otimes \mathcal{M}
$$

The fact that unit $\mathcal{M}$ and $\langle-,-\rangle$ satisfy the duality axioms follows by diagram chase.

D.5.6. Let

$$
\widetilde{\mathbf{1}}_{\mathrm{QCoh}\left(Y_{\mathrm{dR}}\right)} \in \mathrm{QCoh}\left(Y_{\mathrm{dR}}\right)
$$

be the object corresponding to $k_{Y} \in \mathrm{D}-\bmod (Y)$. Note that it satisfies the assumption of Sect. D.2.1 and the corresponding self-duality of $\mathrm{D}-\bmod (Y)$ is $\mathbb{D}_{Y}^{\text {Verdier }}$.

It follows from Lemma D.5.5 that any $\mathcal{M}$ as above satisfies the assumption of Sect. D.2.5

D.5.7. We now claim:

Theorem D.5.8. For $m \in \mathcal{M}^{c}$ the following conditions are equivalent:

(i) The object $m$ is ULA over $\mathrm{D}-\bmod (Y)$;

(ii) For every $\mathcal{F} \in \mathrm{D}-\bmod (Y)^{c}$, the object $\mathcal{F} \otimes m \in \mathcal{M}$ is compact;

(iii) The object $\mathbf{o b l v}(m) \in \mathcal{M}_{Y}$ is compact.

The rest of this subsection is devoted to the proof of this theorem. 
D.5.9. The implication (i) $\Rightarrow$ (ii) follows from Sect. D.1.5 and Lemma D.1.6. To prove (iii) $\Rightarrow$ (ii), it suffices to show that for $\mathcal{F}_{0} \in \mathrm{Q} \operatorname{Coh}(Y)^{c}$, we have

$$
\operatorname{ind}\left(\mathcal{F}_{0}\right) \otimes m \in \mathcal{M}^{c} .
$$

However,

$$
\operatorname{ind}\left(\mathcal{F}_{0}\right) \otimes m \simeq \operatorname{ind}\left(\mathcal{F}_{0} \otimes \operatorname{oblv}(m)\right),
$$

and the assertion follows from the fact that the functor ind preserves compactness.

Proof of (iii) $\Rightarrow$ (i). This is in fact a general assertion. Let $\phi: \mathcal{C} \rightarrow \mathcal{C}_{0}$ be a symmetric monoidal functor that admits a $\mathcal{C}$-linear left adjoint. Let $\mathcal{M}$ be a dualizable $\mathcal{C}$-module category and set

$$
\mathcal{M}_{0}:=\mathcal{C}_{0} \underset{\mathfrak{e}}{\otimes} \mathcal{M} \text {. }
$$

Consider the corresponding functor

$$
\phi_{\mathcal{M}}:=\left(\phi \otimes \operatorname{Id}_{\mathcal{M}}\right): \mathcal{M} \rightarrow \mathcal{M}_{0} ;
$$

it admits a left adjoint, given by $\left(\phi^{L} \otimes \operatorname{Id}_{\mathcal{M}}\right)$.

Consider the inner Hom object $\underline{\operatorname{Hom}}\left(m, m^{\prime}\right) \in \mathcal{C}$. Note that we have

$$
\phi\left(\underline{\operatorname{Hom}}\left(m, m^{\prime}\right)\right) \simeq \underline{\operatorname{Hom}}_{0}\left(\phi_{\mathcal{M}}(m), \phi_{\mathcal{M}}\left(m^{\prime}\right)\right),
$$

where $\underline{\operatorname{Hom}}_{0}(-,-)$ denotes inner Hom in $\mathcal{C}_{0}$.

Assume now that $\phi$ is conservative. It follows from (D.17) and Lemma D.1.6 that if $\phi_{\mathcal{M}}(m) \in \mathcal{M}_{0}$ is ULA over $\mathcal{C}_{0}$, then $m$ is ULA over over $\mathcal{C}$.

We apply this to $\phi$ being

$$
\text { oblv : } \mathrm{D}-\bmod (Y) \rightarrow \mathrm{QCoh}(Y) .
$$

Assumption (iii) says that $\operatorname{oblv}_{Y}(m)$ is compact. It is then automatically ULA over QCoh $(Y)$ by Corollary D.1.7.

The implication (ii) $\Rightarrow$ (iii) follows by

$$
\text { ind } \circ \operatorname{oblv}(m) \simeq \mathrm{D}_{Y} \otimes m
$$

from the following more general assertion:

Proposition D.5.10. Let $m \in \mathcal{M}_{Y}$ be such that $\operatorname{ind}(m) \in \mathcal{M}$ is compact. Then $m$ is compact.

Proof. We need to show that the functor

$$
m^{\prime} \mapsto \mathcal{H}_{o m \mathcal{M}_{Y}}\left(m, m^{\prime}\right)
$$

preserves colimits. We will do so by expressing it via the functor

$$
m^{\prime} \mapsto \mathcal{H} \operatorname{Hom}_{\mathcal{M}}\left(\operatorname{ind}(m), \operatorname{ind}\left(m^{\prime}\right)\right) \simeq \mathcal{H}_{o m_{\mathcal{M}_{Y}}}\left(m, \text { oblv } \circ \operatorname{ind}\left(m^{\prime}\right)\right),
$$

while the latter preserves colimits by assumption.

With no restriction of generality, we can assume that $Y$ is affine.

Consider the formal completion of $Y$ in $Y \times Y$

$$
Y \stackrel{p_{1}}{\leftarrow} Y^{\wedge} \stackrel{p_{2}}{\rightarrow} Y \text {. }
$$

Let $\mathcal{M}_{Y^{\wedge}}$ be the value of $\mathcal{M}$ over $Y^{\wedge}$. Set

$$
\mathcal{M}_{Y^{\wedge}}^{\prime}:=\operatorname{Ind} \operatorname{Coh}\left(Y^{\wedge}\right) \underset{\mathrm{QCoh}\left(Y^{\wedge}\right)}{\otimes} \mathcal{M}_{Y^{\wedge}}
$$

The maps $p_{1}, p_{2}$ define the functors

$$
p_{i}^{*}: \mathcal{M}_{Y} \rightarrow \mathcal{M}_{Y^{\wedge}}
$$

and

$$
\left(p_{i}^{\text {IndCoh }}\right)_{*}: \mathcal{M}_{Y^{\wedge}}^{\prime} \rightarrow \mathcal{M}_{Y} .
$$


Note that the functor

$$
m^{\prime} \mapsto \operatorname{oblv} \circ \operatorname{ind}\left(m^{\prime}\right)
$$

identifies with

$$
m^{\prime} \mapsto\left(p_{1}^{\text {IndCoh }}\right)_{*}\left(\mathrm{D}_{Y} \otimes p_{2}^{*}\left(m^{\prime}\right)\right),
$$

where we regard $\mathrm{D}_{Y}$ as an object of $\operatorname{Ind} \operatorname{Coh}\left(Y^{\wedge}\right)$.

From here it follows oblv $\circ$ ind $\left(m^{\prime}\right)$ carries an action of the algebra $\mathcal{O}_{Y \times Y}$ of functions on $Y \times Y$ (via its action on $\mathrm{D}_{Y}$ ), and we have a functorial identification

$$
m^{\prime} \simeq \mathcal{H}^{\circ} m_{\mathcal{O}_{Y \times Y}}\left(\mathcal{O}_{Y}, \text { oblv } \circ \operatorname{ind}\left(m^{\prime}\right)\right) .
$$

From here, we obtain that $\mathcal{H}_{o m_{\mathcal{M}_{Y}}}\left(m\right.$, oblv $\circ$ ind $\left.\left(m^{\prime}\right)\right)$ carries an action of $\mathcal{O}_{Y \times Y}$ and

$$
\mathcal{H}_{o m} m_{\mathcal{M}_{Y}}\left(m, m^{\prime}\right) \simeq \mathcal{H}_{o} m_{\mathcal{O}_{Y \times Y}}\left(\mathcal{O}_{Y}, \mathcal{H}_{o} m_{\mathcal{M}_{Y}}\left(m, \text { oblv } \circ \operatorname{ind}\left(m^{\prime}\right)\right)\right) \text {. }
$$

Now, the required assertion follows from the fact that the functor

$$
\mathcal{H}_{\text {om }} \mathcal{O}_{Y \times Y}\left(\mathcal{O}_{Y},-\right): \mathcal{O}_{Y \times Y}-\bmod \rightarrow \text { Vect }
$$

commutes with colimits, since $\mathcal{O}_{Y}$ is a compact object of $\mathcal{O}_{Y \times Y}$, the latter because $Y$ is smooth.

This completes the proof of Theorem D.5.8 


\section{REFERENCES}

[AG] D. Arinkin and D. Gaitsgory, Singular support of coherent sheaves, and the geometric Langlands conjecture, joint with D. Arinkin, Selecta Math. N.S. 21 (2015), 1-199.

[Be] A. Beilinson, Constructible sheaves are holonomic, Selecta Mathematica 22 (2016), 1797-1819.

[BFN] , D. Ben-Zvi, J. Francis and D. Nadler, Integral transforms and Drinfeld centers in derived algebraic geometry, J. Amer. Math. Soc. 23 (2010), 909-966.

[BN1] D. Ben-Zvi and D. Nadler, Betti Geometric Langlands, arXiv:1606.08523

[BN2] D. Ben-Zvi and D. Nadler, Non-linear traces, arXiv:1305.7175

[DrGa1] V. Drinfeld and D. Gaitsgory, On some finiteness questions for algebraic stacks, GAFA 23 (2013), $149-294$.

$[\mathrm{DrGa2}]$ V. Drinfeld and D. Gaitsgory, Compact generation of the category of D-modules on the stack of G-bundles on a curve Cambridge Math Journal, 3 (2015), 19-125.

[Ga1] D. Gaitsgory, Outline of the proof of the geometric Langlands conjecture for GL(2), Astérisque 370 (2015), $1-112$.

[Ga2] D. Gaitsgory, From geometric to function-theoretic Langlands (or how to invent shtukas), arXiv:1606.09608

[Ga3] D. Gaitsgory, Semi-infinite intersection cohomology sheaf-II: the Ran space version, arXiv:1708.07205.

[Ga4] D. Gaitsgory, The local and global versions of the Whittaker category, arXiv:1811.02468

[Ga5] D. Gaitsgory, Sheaves of categories and the notion of 1-affineness, Contemporary Mathematics 643 (2015), $1-99$.

[Ga6] D. Gaitsgory, Stability of the ULA property under taking perverse cohomologies, available at: www.math.harvard.edu $\sim$ gaitsgde/GL.

[GaLu] D. Gaitsgory and J. Lurie, Weil's conjecture over function fields-I, Princeton University Press (2019).

[GR1] D. Gaitsgory and N. Rozenblyum, A study in derived algebraic geometry, Vol. 1: Correspondences and Duality, Mathematical surveys and monographs 221 (2017), AMS, Providence, RI.

[GR2] D. Gaitsgory and N. Rozenblyum, A study in derived algebraic geometry, Vol. 2: Deformations, Lie Theory and Formal Geometry, Mathematical surveys and monographs 221 (2017), AMS, Providence, RI.

[GHN] D. Gepner, R. Haugseng and T. Nikolaus, Lax colimits and free fibrations in $\infty$-categories, Doc. Math. 22 (2017), 1225-1266.

[HSS] M. Hoyois, S. Scherotzke and N. Sibilla, Higher traces, noncommutative motives, and the categorified Chern character, Advances in Math. 309 (2017), 97-154.

[KS] M. Kashiwara and P. Schapira, Sheaves on manifolds, Grundlehren der Mathematischen Wissenschaften, 292, Springer-Verlag, Berlin (1990).

[KP1] G. Kondyrev and A. Prihodko, Categorical proof of holomorphic Atiyah-Bott formula, Journal of the Institute of Mathematics of Jussieu (posted online 2018), 1-25, also arXiv: 1607.06345.

[KP2] G. Kondyrev and A. Prihodko, Equivariant Riemann-Roch via formal deformation theory, arXiv:1906.00172

[Laf] V. Lafforgue, Chtoucas pour les groupes réductifs et paramétrisation de Langlands globale, JAMS 31 (2018), $719-891$.

[Lu1] J. Lurie, Higher Topos Theory, Annals of Mathematical Studies, Princeton University Press (2009).

[Lu2] J. Lurie, Higher Algebra, available at: www.math.harvard.edu lurie.

[Lu3] J. Lurie, Spectral Algebraic Geometry, available at: www.math.harvard.edu lurie.

[LZ] Q. Lu and W. Zheng, Duality and nearby cycles over general bases, arXiv: 1712.10216.

[MV] I. Mirkovic and K. Vilonen, Geometric Langlands duality and representations of algebraic groups over commutative rings, Annals of Mathematics 166 (2006), 95-143.

[Ne1] A. Neeman, On the derived category of sheaves on a manifold, Documenta Mathematica 6 (2001), 483-488.

[Ne2] A. Neeman, Non-left-complete derived categories, Math. Research Letters, 18 (2011), 827-832.

[NY] D. Nadler and Z.-W. Yun, Spectral action in Betti geometric Langlands, arXiv:1611.04078

[Ro] N. Rozenblyum, Filtered colimits of $\infty$-categories, available at: http://www.math.harvard.edu/ gaitsgde/GL/colimits.pdf

[St] J. Stasheff, Homotopy associativity of H-spaces I, II., Trans. Amer. Math. Soc. 108 1963, 275 - 312.

[TV] B. Toen and G. Vezzosi, Caractères de Chern, traces équivariantes et géométrie algébrique dérivée, Selecta Math. (N.S.), 21 (2015), 449-554.

[Va] Y. Varshavsky, Local terms for transversal intersections, arXiv:2003.06815

[Xue] C. Xue, Finiteness of cohomologies of stacks of shtukas as modules over Hecke algebras, and applications, arXiv:1811.09513

D.G I4: Department of Mathematics, Harvard University;

D.K.: Einstein School of Mathematics, Hebrew University of Jerusalem;

N.R.: Department of Mathematics, The University of Chicago;

Y.V.: Einstein School of Mathematics, Hebrew University of Jerusalem.

${ }^{14}$ Corresponding author. Full address: Department of Mathematics, Harvard University, 1 Oxford str, Cambridge MA 02138, USA 